Cochrane Database of Systematic Reviews

\title{
Electroconvulsive therapy for treatment-resistant schizophrenia
} (Review)

Sinclair DJM, Zhao S, Qi F, Nyakyoma K, Kwong JSW, Adams CE

Sinclair DJM, Zhao S, Qi F, Nyakyoma K, Kwong JSW, Adams CE.

Electroconvulsive therapy for treatment-resistant schizophrenia.

Cochrane Database of Systematic Reviews 2019, Issue 3. Art. No.: CD011847.

DOI: 10.1002/14651858.CD011847.pub2.

www.cochranelibrary.com 
TABLE OF CONTENTS

HEADER

ABSTRACT

PLAIN LANGUAGE SUMMARY

SUMMARY OF FINDINGS

BACKGROUND

OBJECTIVES

METHODS

RESULTS

Figure 1.

Figure 2.

Figure 3.

DISCUSSION

AUTHORS' CONCLUSIONS

ACKNOWLEDGEMENTS

REFERENCES

CHARACTERISTICS OF STUDIES

DATA AND ANALYSES

Analysis 1.1. Comparison 1 ECT plus standard care versus sham-ECT plus standard care, Outcome 1 Mental state - total score (BPRS, high = poor) - short term.

Analysis 1.2. Comparison 1 ECT plus standard care versus sham-ECT plus standard care, Outcome 2 Service use - number readmitted - short term.

Analysis 2.1. Comparison 2 ECT plus standard care versus antipsychotics plus standard care, Outcome 1 Response to treatment - clinically important response (BPRS reducing rate $\geq 50 \%$ ) - medium term.

Analysis 2.2. Comparison 2 ECT plus standard care versus antipsychotics plus standard care, Outcome 2 Mental state - total score (BPRS, high = poor) - short term.

Analysis 2.3. Comparison 2 ECT plus standard care versus antipsychotics plus standard care, Outcome 3 Mental state - total score (BPRS, high = poor) - medium term (skewed data).

Analysis 2.4. Comparison 2 ECT plus standard care versus antipsychotics plus standard care, Outcome 4 Mental state - specific symptom score (BPRS, high = poor) - short term.

Analysis 2.5. Comparison 2 ECT plus standard care versus antipsychotics plus standard care, Outcome 5 Mental state - specific symptom score (BPRS, high = poor) - medium term.

Analysis 2.6. Comparison 2 ECT plus standard care versus antipsychotics plus standard care, Outcome 6 Adverse events - total score (TESS, high = poor).

Analysis 3.1. Comparison 3 ECT plus standard care versus standard care, Outcome 1 Response to treatment - clinically important response (as defined by each study).

Analysis 3.2. Comparison 3 ECT plus standard care versus standard care, Outcome 2 Cognitive functioning - memory deterioration - short term.

Analysis 3.3. Comparison 3 ECT plus standard care versus standard care, Outcome 3 Cognitive functioning - specific symptom score (WCST, high = poor, other than correct number) - medium term.

Analysis 3.4. Comparison 3 ECT plus standard care versus standard care, Outcome 4 Cognitive functioning - specific symptom score (WCST, high = poor) - medium term (skewed data).

Analysis 3.5. Comparison 3 ECT plus standard care versus standard care, Outcome 5 Cognitive functioning - memory (WMS, high = good) - short term.

Analysis 3.6. Comparison 3 ECT plus standard care versus standard care, Outcome 6 Cognitive functioning - memory (WMS, high = good) - medium term.

Analysis 3.7. Comparison 3 ECT plus standard care versus standard care, Outcome 7 Satisfaction and acceptability of treatment - leaving the study early - medium term.

Analysis 3.8. Comparison 3 ECT plus standard care versus standard care, Outcome 8 Mental state - total score (BPRS, high = poor).

Analysis 3.9. Comparison 3 ECT plus standard care versus standard care, Outcome 9 Mental state - total score (BPRS, high = poor) - medium term (skewed data).

Analysis 3.10. Comparison 3 ECT plus standard care versus standard care, Outcome 10 Mental state - total score (MMSE, high = good) - medium term. 
Analysis 3.11. Comparison 3 ECT plus standard care versus standard care, Outcome 11 Mental state - total score (PANSS, high = poor).

Analysis 3.12. Comparison 3 ECT plus standard care versus standard care, Outcome 12 Mental state - specific symptom score (BPRS, high = poor) - short term.

Analysis 3.13. Comparison 3 ECT plus standard care versus standard care, Outcome 13 Mental state - specific symptom score (BPRS, high = poor) - medium term.

Analysis 3.14. Comparison 3 ECT plus standard care versus standard care, Outcome 14 Mental state - specific symptom score (PANSS, high $=$ poor) - short term.

Analysis 3.15. Comparison 3 ECT plus standard care versus standard care, Outcome 15 Mental state - specific symptom score (PANSS, high = poor) - short term (skewed data).

Analysis 3.16. Comparison 3 ECT plus standard care versus standard care, Outcome 16 Mental state - specific symptom score (PANSS, high = poor) - medium term.

Analysis 3.17. Comparison 3 ECT plus standard care versus standard care, Outcome 17 Mental state - specific symptom score (PANSS, high = poor) - medium term (skewed data).

Analysis 3.18. Comparison 3 ECT plus standard care versus standard care, Outcome 18 Mental state - specific symptom score (SAPS, high = poor) - short term.

Analysis 3.19. Comparison 3 ECT plus standard care versus standard care, Outcome 19 Mental state - specific symptom score (SANS, high = poor) - short term.

Analysis 3.20. Comparison 3 ECT plus standard care versus standard care, Outcome 20 General functioning - total score (GAF, high $=$ good).

Analysis 3.21. Comparison 3 ECT plus standard care versus standard care, Outcome 21 Adverse events - general - medium term.

Analysis 3.22. Comparison 3 ECT plus standard care versus standard care, Outcome 22 Adverse events - total score (TESS, high = poor).

Analysis 3.23. Comparison 3 ECT plus standard care versus standard care, Outcome 23 Adverse events - specific symptom short term.

Analysis 3.24. Comparison 3 ECT plus standard care versus standard care, Outcome 24 Adverse events - specific symptom medium term.

Analysis 3.25. Comparison 3 ECT plus standard care versus standard care, Outcome 25 Adverse events - specific symptom score (TESS, high = poor) - short term (skewed data).

Analysis 3.26. Comparison 3 ECT plus standard care versus standard care, Outcome 26 Adverse events - specific symptom score (TESS, high = poor) - medium term.

Analysis 3.27. Comparison 3 ECT plus standard care versus standard care, Outcome 27 Adverse events - specific symptom score (TESS, high = poor) - medium term (skewed data).

Analysis 4.1. Comparison 4 ECT alone versus antipsychotic alone (flupenthixol), Outcome 1 Mental state - total score (BPRS, high = poor) - medium term.

Analysis 4.2. Comparison 4 ECT alone versus antipsychotic alone (flupenthixol), Outcome 2 Mental state - total score (MMSE, high = good) - medium term.

Analysis 4.3. Comparison 4 ECT alone versus antipsychotic alone (flupenthixol), Outcome 3 General functioning - total score $(\mathrm{GAF}$, high $=$ good $)-$ medium term.

Analysis 5.1. Comparison 5 SUBGROUP: ECT plus standard care versus standard care (TEST FOR DIFFERENCE BETWEEN SHORT/ LONG COURSE OF ECT), Outcome 1 Response to treatment - clinically significant response (as defined by each study) - medium term.

Analysis 6.1. Comparison 6 SENSITIVITY ANALYSIS: ECT plus standard care versus standard care (ASSUMPTIONS FOR LOST BINARY DATA), Outcome 1 Response to treatment - clinically significant response (as defined by each study) - medium term. .. Analysis 7.1. Comparison 7 SENSITIVITY ANALYSIS: ECT plus standard care versus standard care (FIXED-EFFECT AND RANDOMEFFECTS MODEL), Outcome 1 Response to treatment - clinically significant response (as defined by each study) - medium term.

CONTRIBUTIONS OF AUTHORS

DECLARATIONS OF INTEREST

SOURCES OF SUPPORT

DIFFERENCES BETWEEN PROTOCOL AND REVIEW

NOTES

INDEX TERMS 
[Intervention Review]

\section{Electroconvulsive therapy for treatment-resistant schizophrenia}

Diarmid JM Sinclair ${ }^{1}$, Sai Zhao², Fang Qi², Kazare Nyakyoma ${ }^{3}$, Joey SW Kwong ${ }^{4}$, Clive E Adams 5

${ }^{1}$ General Adult Psychiatry, Nottinghamshire Healthcare NHS Trust, Worksop, UK. ${ }^{2}$ Systematic Review Solutions Ltd, The Ingenuity Centre, The University of Nottingham, Nottingham, UK. ${ }^{3}$ Derby City Acute Mental Health, Derbyshire Healthcare Foundation NHS Trust, Derby DE1 2QY, UK. " Department of Health Policy and Department of Clinical Epidemiology, National Center for Child Health and Development, Setagaya-ku, Japan. ${ }^{5}$ Cochrane Schizophrenia Group, The University of Nottingham, Nottingham, UK

Contact address: Diarmid JM Sinclair, General Adult Psychiatry, Nottinghamshire Healthcare NHS Trust, Bassetlaw Hospital, Worksop, South Yorkshire, S81 0BD, UK. diarmid.sinclair@nhs.net.

Editorial group: Cochrane Schizophrenia Group

Publication status and date: New, published in Issue 3, 2019.

Citation: Sinclair DJM, Zhao S, Qi F, Nyakyoma K, Kwong JSW, Adams CE. Electroconvulsive therapy for treatment-resistant schizophrenia. Cochrane Database of Systematic Reviews 2019, Issue 3. Art. No.: CD011847. DOI: 10.1002/14651858.CD011847.pub2.

Copyright ( 2019 The Cochrane Collaboration. Published by John Wiley \& Sons, Ltd.

\section{A B S T R A C T}

\section{Background}

Electroconvulsive therapy (ECT) involves the induction of a seizure by the administration of an electrical stimulus via electrodes usually placed bilaterally on the scalp and was introduced as a treatment for schizophrenia in 1938. However, ECT is a controversial treatment with concerns about long-term side effects such a memory loss. Therefore, it is important to determine its clinical efficacy and safety for people with schizophrenia who are not responding to their treatment.

\section{Objectives}

Our primary objective was to assess the effects (benefits and harms) of ECT for people with treatment-resistant schizophrenia.

Our secondary objectives were to determine whether ECT produces a differential response in people: who are treated with unilateral compared to bilateral ECT; who have had a long (more than 12 sessions) or a short course of ECT; who are given continuation or maintenance ECT; who are diagnosed with well-defined treatment-resistant schizophrenia as opposed to less rigorously defined treatment-resistant schizophrenia (who would be expected to have a greater affective component to their illness).

\section{Search methods}

We searched the Cochrane Schizophrenia Group's Study-Based Register of Trials including clinical trial registries on 9 September 2015 and 4 August 2017. There were no limitations on language, date, document type, or publication status for the inclusion of records in the register. We also inspected references of all the included records to identify further relevant studies.

\section{Selection criteria}

Randomised controlled trials investigating the effects of ECT in people with treatment-resistant schizophrenia.

\section{Data collection and analysis}

Two review authors independently extracted data. For binary outcomes, we calculated the risk ratio (RR) and its $95 \%$ confidence intervals (Cls), on an intention-to-treat basis. For continuous data, we estimated the mean difference (MD) between the groups and its $95 \%$ Cls. We employed the fixed-effect model for all analyses. We assessed risk of bias for the included studies and created 'Summary of findings' tables using the GRADE framework. 


\section{Main results}

We included 15 studies involving 1285 participants (1264 completers with an average age of 18 to 46 years) with treatment-resistant schizophrenia. We rated most studies $(14 / 15,93.3 \%)$ as at high risk of bias due to issues related to the blinding of participants and personnel. Our main outcomes of interest were: (i) clinically important response to treatment; (ii) clinically important change in cognitive functioning; (iii) leaving the study early; (iv) clinically important change in general mental state; (v) clinically important change in general functioning; (vi) number hospitalised; and (vii) death. No trial reported data on death.

The included trials reported useable data for four comparisons: ECT plus standard care compared with sham-ECT added to standard care; ECT plus standard care compared with antipsychotic added to standard care; ECT plus standard care compared with standard care; and ECT alone compared with antipsychotic alone.

For the comparison ECT plus standard care versus sham-ECT plus standard care, only average endpoint BPRS (Brief Psychiatric Rating Scale) scores from one study were available for mental state; no clear difference between groups was observed (short term; MD 3.60, 95\% $\mathrm{Cl}-3.69$ to 10.89; participants $=25$; studies $=1$; very low-quality evidence). One study reported data for service use, measured as number readmitted; there was a clear difference favouring the ECT group (short term; RR $0.29,95 \% \mathrm{Cl} 0.10$ to 0.85 ; participants = 25; studies = 1; low-quality evidence).

When ECT plus standard care was compared with antipsychotics (clozapine) plus standard care, data from one study showed no clear difference for clinically important response to treatment (medium term; RR 1.23, 95\% Cl 0.95 to 1.58; participants = 162; studies = 1; lowquality evidence). Clinically important change in mental state data were not available, but average endpoint BPRS scores were reported. A positive effect for the ECT group was found (short-term BPRS; MD -5.20, 95\% Cl -7.93 to -2.47; participants = 162; studies = 1; very lowquality evidence).

When ECT plus standard care was compared with standard care, more participants in the ECT group had a clinically important response (medium term; RR 2.06, 95\% Cl 1.75 to 2.42; participants = 819; studies = 9; moderate-quality evidence). Data on clinically important change in cognitive functioning were not available, but data for memory deterioration were reported. Results showed that adding ECT to standard care may increase the risk of memory deterioration (short term; RR $27.00,95 \% \mathrm{Cl} 1.67$ to 437.68 ; participants $=72$; studies = 1 ; very low-quality evidence). There were no clear differences between groups in satisfaction and acceptability of treatment, measured as leaving the study early (medium term; RR $1.18,95 \% \mathrm{Cl} 0.38$ to 3.63; participants $=354$; studies $=3$; very low-quality evidence). Only average endpoint scale scores were available for mental state (BPRS) and general functioning (Global Assessment of Functioning). There were clear differences in scores, favouring ECT group for mental state (medium term; MD $-11.18,95 \% \mathrm{Cl}-12.61$ to -9.76 ; participants = 345; studies = 2; low-quality evidence) and general functioning (medium term; MD 10.66, 95\% Cl 6.98 to 14.34; participants = 97; studies = 2; very lowquality evidence).

For the comparison ECT alone versus antipsychotics (flupenthixol) alone, only average endpoint scale scores were available for mental state and general functioning. Mental state scores were similar between groups (medium-term BPRS; MD - $0.93,95 \% \mathrm{Cl}-6.95$ to 5.09 ; participants = 30; studies = 1; very low-quality evidence); general functioning scores were also similar between groups (medium-term Global Assessment of Functioning; MD - $0.66,95 \% \mathrm{Cl}-3.60$ to 2.28; participants = 30; studies = 1; very low-quality evidence).

\section{Authors' conclusions}

Moderate-quality evidence indicates that relative to standard care, ECT has a positive effect on medium-term clinical response for people with treatment-resistant schizophrenia. However, there is no clear and convincing advantage or disadvantage for adding ECT to standard care for other outcomes. The available evidence was also too weak to indicate whether adding ECT to standard care is superior or inferior to adding sham-ECT or other antipsychotics to standard care, and there was insufficient evidence to support or refute the use of ECT alone. More good-quality evidence is needed before firm conclusions can be made.

\section{PLAIN LANGUAGE SUMMARY}

\section{Electroconvulsive therapy for treatment-resistant schizophrenia}

\section{Review question}

Is electroconvulsive therapy (ECT) a safe and effective treatment for people with schizophrenia who have not responded to their previous treatment?

\section{Background}

Electroconvulsive therapy involves the induction of a seizure by the administration of an electrical stimulus via electrodes usually placed bilaterally on the scalp. Electroconvulsive therapy was once widely used as a treatment for people with schizophrenia, but its use in now reduced due to the development of antipsychotic medications and concerns regarding possible long-term adverse effects of ECT.

\section{Methods and results}


Searches for randomised clinical trials (a type of study in which participants are assigned to one of two or more treatment groups using a random method) were conducted in 2015 and updated in 2017. We included 15 studies involving 1285 people with treatment-resistant schizophrenia. The age range of participants was 18 to 46 years. One study compared adding ECT to standard care with adding sham-ECT (non-active ECT); one study compared adding ECT to standard care with adding additional antipsychotic to standard care; and 12 studies compared adding ECT to standard care with standard care. One study compared ECT as the sole treatment with antipsychotics as the sole treatment.

Our main outcomes of interest were clinically important response to treatment, cognitive functioning, leaving the study early, mental state, general functioning, number hospitalised, and death. None of the included studies reported data for death. The quality of the evidence for each main outcome was mostly very low or low, with the quality of the evidence rated as moderate for only one outcome. This was mostly due to issues with the way the studies were conducted (e.g. participants were not blinded to treatment) and small sample sizes.

For the comparison of adding ECT to standard care versus standard care, moderate-quality evidence indicates that adding ECT to standard care may have some beneficial effect on clinical response to treatment. Very low-quality evidence indicates that adding ECT to standard care may increase the risk of short-term memory problems and could have a positive effect on Global Assessment of Functioning scores. Low-quality evidence indicates that adding ECT to standard care may have a positive effect on Brief Psychiatric Rating Scale scores.

The evidence for the other comparisons was also of low or very low quality, and overall no clear differences between the treatment groups were found.

\section{Conclusions}

We found moderate-quality evidence that adding ECT to standard care has a positive effect on clinical response when compared with standard care. The currently available evidence was too weak to clearly demonstrate that adding ECT to standard treatment is associated with benefits or harm for our other outcomes. There is also a lack of evidence on the effects and safety of adding ECT to standard care compared with sham-ECT or additional antipsychotics and inadequate evidence regarding the use of ECT alone. 
SUMMARY OF FINDINGS

\section{Summary of findings for the main comparison. ECT plus standard care versus sham-ECT plus standard care for treatment-resistant schizophrenia}

ECT plus standard care versus sham-ECT plus standard care for treatment-resistant schizophrenia

Patient or population: people with treatment-resistant schizophrenia

Settings: hospital

Intervention: $\mathrm{ECT}$ plus standard care

Comparison: sham-ECT plus standard care

\begin{tabular}{|c|c|c|c|c|c|c|}
\hline \multirow[t]{2}{*}{ Outcomes } & \multicolumn{2}{|c|}{$\begin{array}{l}\text { Anticipated absolute effects* } \\
(95 \% \mathrm{Cl})\end{array}$} & \multirow{2}{*}{$\begin{array}{l}\text { Relative ef- } \\
\text { fect } \\
(95 \% \mathrm{CI})\end{array}$} & \multirow{2}{*}{$\begin{array}{l}\text { No. of partic- } \\
\text { ipants } \\
\text { (studies) }\end{array}$} & \multirow{2}{*}{$\begin{array}{l}\text { Quality of the } \\
\text { evidence } \\
\text { (GRADE) }\end{array}$} & \multirow[t]{2}{*}{ Comments } \\
\hline & $\begin{array}{l}\text { Risk with } \\
\text { sham-ECT }\end{array}$ & $\begin{array}{l}\text { Risk with ECT } \\
\text { (add-on) }\end{array}$ & & & & \\
\hline $\begin{array}{l}\text { Response to treatment } \\
\text { Clinically important response to treatment as de- } \\
\text { fined by each study }\end{array}$ & See comment & See comment & See comment & See comment & See comment & $\begin{array}{l}\text { No studies reported this out- } \\
\text { come, so there is no evidence } \\
\text { to support or refute benefits of } \\
\text { the intervention. }\end{array}$ \\
\hline $\begin{array}{l}\text { Cognitive functioning } \\
\text { Clinically important change in cognitive function- } \\
\text { ing as defined by each study }\end{array}$ & See comment & See comment & See comment & See comment & See comment & $\begin{array}{l}\text { No studies reported this out- } \\
\text { come, so there is no evidence } \\
\text { to support or refute benefits of } \\
\text { the intervention. }\end{array}$ \\
\hline $\begin{array}{l}\text { Satisfaction and acceptability of treatment - } \\
\text { leaving the study early }\end{array}$ & See comment & See comment & See comment & See comment & See comment & $\begin{array}{l}\text { No studies reported this out- } \\
\text { come, so there is no evidence } \\
\text { to support or refute benefits of } \\
\text { the intervention. }\end{array}$ \\
\hline $\begin{array}{l}\text { Mental state (short term) - total scores (BPRS, } \\
\text { high = poor) } \\
\text { Follow-up: } 4 \text { weeks }\end{array}$ & $\begin{array}{l}\text { The mean } \\
\text { mental state } \\
\text { - average } \\
\text { scores (BPRS, } \\
\text { high = poor, } \\
\text { short term) } \\
\text { was } \mathbf{4 0 . 4} \text {. }\end{array}$ & $\begin{array}{l}\text { MD } 3.60 \text { high- } \\
\text { er }(3.69 \text { lower } \\
\text { to } 10.89 \text { high- } \\
\text { er) }\end{array}$ & - & 25 & $\begin{array}{l}\oplus \ominus \ominus \ominus \\
\text { very low } 1,2\end{array}$ & $\begin{array}{l}\text { Data for predefined outcome } \\
\text { 'clinically important change' } \\
\text { not reported. }\end{array}$ \\
\hline $\begin{array}{l}\text { General functioning } \\
\text { Clinically important change in general function- } \\
\text { ing as defined by each study }\end{array}$ & See comment & See comment & See comment & See comment & See comment & $\begin{array}{l}\text { No studies reported this out- } \\
\text { come, so there is no evidence }\end{array}$ \\
\hline
\end{tabular}

Clinically important change in general function-

Anticipated absolute effects*

Risk with $\quad$ Risk with ECT

\section{Response to treatme}

Clinically important response to treatment as de-

Clinically important change in cognitive function-

Satisfaction and acceptability of treatment

leaving the study early

Mental state (short term) - total scores (BPRS, 


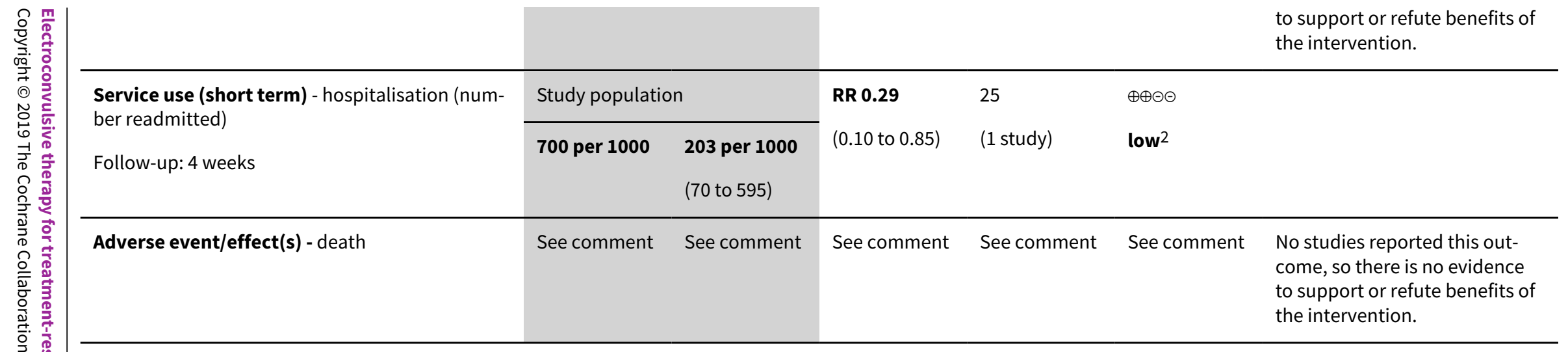

${ }^{*}$ The risk in the intervention group (and its 95\% confidence interval) is based on the assumed risk in the comparison group and the relative effect of the intervention (and its $95 \% \mathrm{Cl}$ ).

BPRS: Brief Psychiatric Rating Scale; Cl: confidence interval; ECT: electroconvulsive therapy; MD: mean difference; RR: risk ratio

GRADE Working Group grades of evidence

High quality: we are very confident that the true effect lies close to that of the estimate of the effect.

Moderate quality: we are moderately confident in the effect estimate; the true effect is likely to be close to the estimate of effect, but there is a possibility that it is substantially different.

Low quality: our confidence in the effect estimate is limited; the true effect may be substantially different from the estimate of the effect.

Very low quality: we have very little confidence in the effect estimate; the true effect is likely to be substantially different from the estimate of effect.

1Downgraded by one level due to indirectness: scores from scale were employed as a surrogate index of the intended outcome.

2Downgraded by two levels due to imprecision: very small sample size.

\section{Summary of findings 2. ECT plus standard care versus antipsychotics plus standard care for treatment-resistant schizophrenia}

ECT plus standard care versus antipsychotics plus standard care for treatment-resistant schizophrenia

Patient or population: people with treatment-resistant schizophrenia

Settings: hospital

Intervention: $\mathrm{ECT}$ plus ziprasidone

Comparison: clozapine plus ziprasidone

\begin{tabular}{ll|lll}
\hline Outcomes & $\begin{array}{l}\text { Anticipated absolute effects* } \\
(95 \% \mathrm{Cl})\end{array}$ & $\begin{array}{l}\text { Relative ef- } \\
\text { fect }\end{array}$ & $\begin{array}{l}\text { No. of partic- } \\
\text { ipants } \\
\text { (studies) }\end{array}$ & $\begin{array}{l}\text { Quality of the } \\
\text { evidence }\end{array}$ \\
(GRADE)
\end{tabular}




\begin{tabular}{|c|c|c|c|c|c|c|}
\hline & $\begin{array}{l}\text { Risk with an- } \\
\text { tipsychotics } \\
\text { (clozapine) }\end{array}$ & $\begin{array}{l}\text { Risk with ECT } \\
\text { (add-on) }\end{array}$ & & & & \\
\hline \multirow{2}{*}{$\begin{array}{l}\text { Response to treatment (medium term) } \\
\text { Clinically important response to treatment as } \\
\text { defined by each study } \\
\text { Follow-up: } 8 \text { weeks }\end{array}$} & \multicolumn{2}{|l|}{ Study population } & \multirow{2}{*}{$\begin{array}{l}\text { RR } 1.23 \\
\text { (0.95 to } 1.58)\end{array}$} & \multirow{2}{*}{$\begin{array}{l}162 \\
\text { (1 study) }\end{array}$} & \multirow{2}{*}{$\begin{array}{l}\oplus \oplus \ominus \ominus \\
\text { low } 1,3\end{array}$} & \\
\hline & 543 per 1000 & $\begin{array}{l}\mathbf{6 6 8} \text { per } \mathbf{1 0 0 0} \\
\text { (516 to } 858)\end{array}$ & & & & \\
\hline $\begin{array}{l}\text { Cognitive functioning } \\
\text { Clinically important change in cognitive func- } \\
\text { tioning as defined by each study }\end{array}$ & See comment & See comment & See comment & See comment & See comment & $\begin{array}{l}\text { No studies reported this out- } \\
\text { come, so there is no evidence } \\
\text { to support or refute benefits of } \\
\text { the intervention. }\end{array}$ \\
\hline $\begin{array}{l}\text { Satisfaction and acceptability of treatment - } \\
\text { leaving the study early }\end{array}$ & See comment & See comment & See comment & See comment & See comment & $\begin{array}{l}\text { No studies reported this out- } \\
\text { come, so there is no evidence } \\
\text { to support or refute benefits of } \\
\text { the intervention. }\end{array}$ \\
\hline $\begin{array}{l}\text { Mental state (short term) - total scores (BPRS, } \\
\text { high = poor) } \\
\text { Follow-up: } 4 \text { weeks }\end{array}$ & $\begin{array}{l}\text { The mean men- } \\
\text { tal state - av- } \\
\text { erage scores } \\
\text { (BPRS, high } \\
=\text { poor, short } \\
\text { term) was } \mathbf{4 4 . 7} \text {. }\end{array}$ & $\begin{array}{l}\text { MD } 5.20 \text { low- } \\
\text { er ( } 7.93 \text { to } \\
2.47 \text { lower })\end{array}$ & - & $\begin{array}{l}162 \\
\text { (1 study) }\end{array}$ & $\begin{array}{l}\oplus \odot \Theta \Theta \\
\text { very low } 1,2,3\end{array}$ & $\begin{array}{l}\text { Data for predefined outcome } \\
\text { 'clinically important change' } \\
\text { not reported. }\end{array}$ \\
\hline $\begin{array}{l}\text { General functioning } \\
\text { Clinically important change in general func- } \\
\text { tioning as defined by each study }\end{array}$ & See comment & See comment & See comment & See comment & See comment & $\begin{array}{l}\text { No studies reported this out- } \\
\text { come, so there is no evidence } \\
\text { to support or refute benefits of } \\
\text { the intervention. }\end{array}$ \\
\hline Service use - hospitalisation & See comment & See comment & See comment & See comment & See comment & $\begin{array}{l}\text { No studies reported this out- } \\
\text { come, so there is no evidence } \\
\text { to support or refute benefits of } \\
\text { the intervention. }\end{array}$ \\
\hline Adverse event/effect(s) - death & See comment & See comment & See comment & See comment & See comment & $\begin{array}{l}\text { No studies reported this out- } \\
\text { come, so there is no evidence } \\
\text { to support or refute benefits of } \\
\text { the intervention. }\end{array}$ \\
\hline
\end{tabular}

${ }^{\star}$ The risk in the intervention group (and its $95 \%$ confidence interval) is based on the assumed risk in the comparison group and the relative effect of the intervention (and its $95 \% \mathrm{Cl}$ ).

BPRS: Brief Psychiatric Rating Scale; Cl: confidence interval; ECT: electroconvulsive therapy; MD: mean difference; RR: risk ratio 


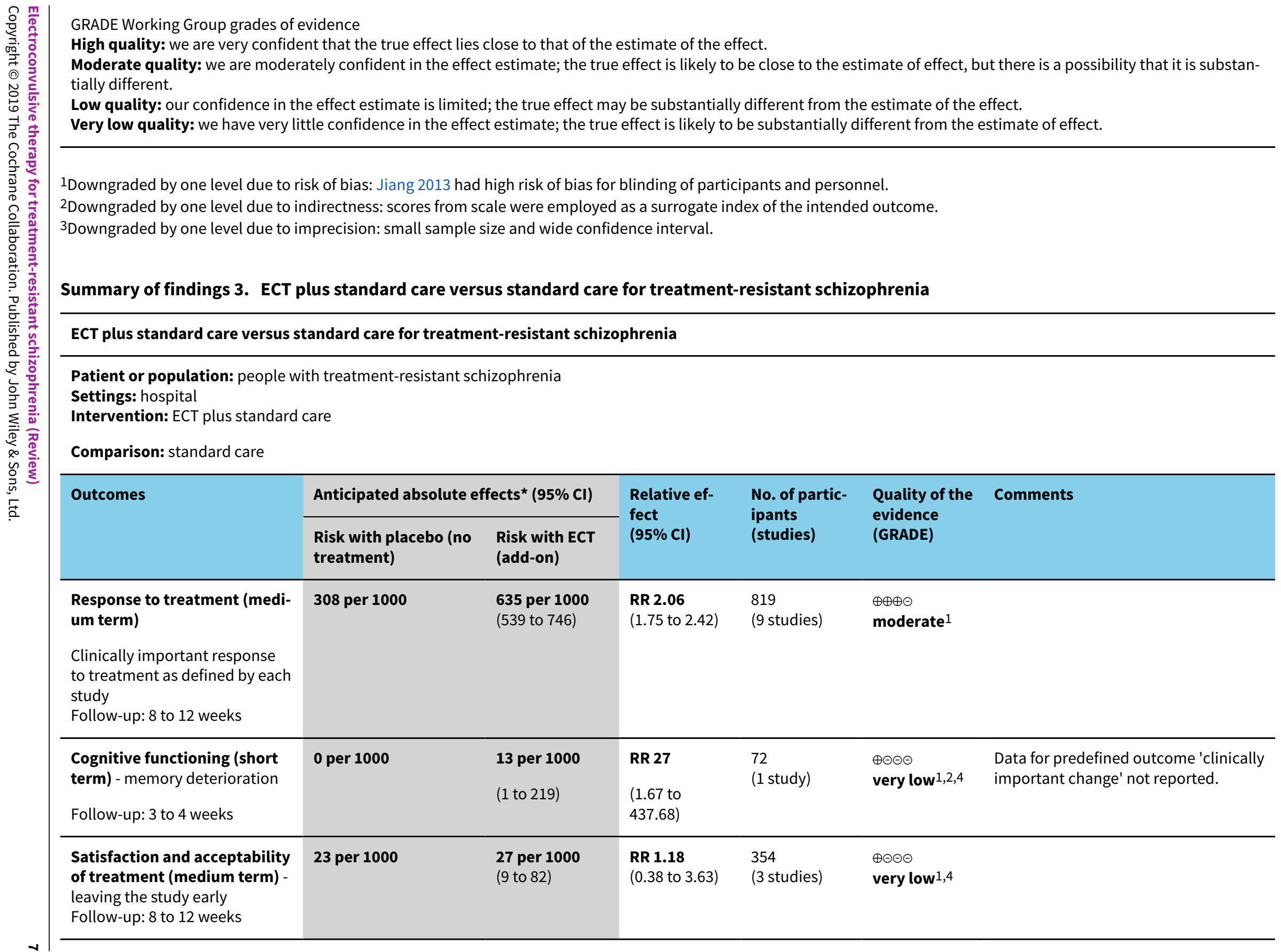




\begin{tabular}{|c|c|c|c|c|c|c|c|}
\hline 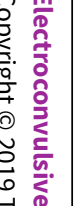 & $\begin{array}{l}\text { Mental state (medium term) - } \\
\text { total scores (BPRS, high = poor) } \\
\text { Follow-up: } 8 \text { to } 12 \text { weeks }\end{array}$ & $\begin{array}{l}\text { The mean mental } \\
\text { state - average scores } \\
\text { (BPRS, high = poor, } \\
\text { medium term) was } \\
\text { 33.4. }\end{array}$ & $\begin{array}{l}\text { MD } 11.18 \text { low- } \\
\text { er }(12.61 \text { to } 9.76 \\
\text { lower })\end{array}$ & - & $\begin{array}{l}345 \\
\text { (2 studies) }\end{array}$ & $\begin{array}{l}\oplus \oplus \ominus \ominus \\
\text { low }^{1,2}\end{array}$ & $\begin{array}{l}\text { Data for predefined outcome 'clinically } \\
\text { important change' not reported. }\end{array}$ \\
\hline $\mid$ & $\begin{array}{l}\text { General functioning (medium } \\
\text { term) - average scores (GAF, } \\
\text { high = good) } \\
\text { Follow-up: } 12 \text { weeks to } 6 \\
\text { months }\end{array}$ & $\begin{array}{l}\text { The mean mental state } \\
\text { - average scores (GAF, } \\
\text { high = good, medium } \\
\text { term) was } \mathbf{4 7 . 3} \text {. }\end{array}$ & $\begin{array}{l}\text { MD } 10.66 \text { high- } \\
\text { er }(6.98 \text { to } 14.34 \\
\text { higher) }\end{array}$ & - & $\begin{array}{l}97 \\
\text { (2 studies) }\end{array}$ & $\begin{array}{l}\oplus \ominus \Theta \odot \\
\text { very } \\
\text { low } 1,2,3,5\end{array}$ & $\begin{array}{l}\text { Data for predefined outcome 'clinically } \\
\text { important change' not reported. }\end{array}$ \\
\hline 黛. & Service use - hospitalisation & See comment & See comment & See comment & See comment & See comment & $\begin{array}{l}\text { No studies reported this outcome, so } \\
\text { there is no evidence to support or re- } \\
\text { fute benefits of the intervention. }\end{array}$ \\
\hline . & $\begin{array}{l}\text { Adverse event/effect(s) - } \\
\text { death }\end{array}$ & See comment & See comment & See comment & See comment & See comment & $\begin{array}{l}\text { No studies reported this outcome, so } \\
\text { there is no evidence to support or re- } \\
\text { fute benefits of the intervention. }\end{array}$ \\
\hline & \multicolumn{7}{|c|}{$\begin{array}{l}\text { *The risk in the intervention group (and its 95\% confidence interval) is based on the assumed risk in the comparison group and the relative effect of the intervention (and } \\
\text { its 95\% CI). } \\
\text { BPRS: Brief Psychiatric Rating Scale; CI: confidence interval; ECT: electroconvulsive therapy; GAF: Global Assessment of Functioning; MD: mean difference; RR: risk ratio }\end{array}$} \\
\hline & \multicolumn{7}{|c|}{$\begin{array}{l}\text { GRADE Working Group grades of evidence } \\
\text { High quality: we are very confident that the true effect lies close to that of the estimate of the effect. } \\
\text { Moderate quality: we are moderately confident in the effect estimate; the true effect is likely to be close to the estimate of effect, but there is a possibility that it is substan- } \\
\text { tially different. } \\
\text { Low quality: our confidence in the effect estimate is limited; the true effect may be substantially different from the estimate of the effect. } \\
\text { Very low quality: we have very little confidence in the effect estimate; the true effect is likely to be substantially different from the estimate of effect. }\end{array}$} \\
\hline
\end{tabular}

1Downgraded by one level due to risk of bias: high risk of bias with blinding of participants and personnel.

2Downgraded by one level due to indirectness: scores from scale were employed as a surrogate index of the intended outcome.

3 Downgraded by one level due to heterogeneity.

${ }^{4}$ Downgraded by two levels due to imprecision: low event rate.

${ }^{5}$ Downgraded by one level due to imprecision: small sample size.

\section{Summary of findings 4. ECT alone versus antipsychotics for treatment-resistant schizophrenia}

ECT alone versus antipsychotics for treatment-resistant schizophrenia

Patient or population: people with treatment-resistant schizophrenia 
Settings: hospital

Intervention: $\mathrm{ECT}$

Comparison: Flupenthixol

\begin{tabular}{|c|c|c|c|c|c|c|}
\hline \multirow[t]{2}{*}{ Outcomes } & \multicolumn{2}{|c|}{$\begin{array}{l}\text { Anticipated absolute effects }{ }^{\star}(95 \% \\
\mathrm{CI})\end{array}$} & \multirow{2}{*}{$\begin{array}{l}\text { Relative ef- } \\
\text { fect } \\
(95 \% \mathrm{Cl})\end{array}$} & \multirow{2}{*}{$\begin{array}{l}\text { No. of partic- } \\
\text { ipants } \\
\text { (studies) }\end{array}$} & \multirow{2}{*}{$\begin{array}{l}\text { Quality of the } \\
\text { evidence } \\
\text { (GRADE) }\end{array}$} & \multirow[t]{2}{*}{ Comments } \\
\hline & $\begin{array}{l}\text { Risk with an- } \\
\text { tipsychotics (flu- } \\
\text { penthixol) }\end{array}$ & $\begin{array}{l}\text { Risk with ECT } \\
\text { (alone) }\end{array}$ & & & & \\
\hline $\begin{array}{l}\text { Response to treatment } \\
\text { Clinically significant response to treat- } \\
\text { ment as defined by each study }\end{array}$ & See comment & See comment & See comment & See comment & See comment & $\begin{array}{l}\text { No studies reported this out- } \\
\text { come, so there is no evidence to } \\
\text { support or refute benefits of the } \\
\text { intervention. }\end{array}$ \\
\hline $\begin{array}{l}\text { Cognitive functioning } \\
\text { Clinically important change in cognitive } \\
\text { functioning as defined by each study }\end{array}$ & See comment & See comment & See comment & See comment & See comment & $\begin{array}{l}\text { No studies reported this out- } \\
\text { come, so there is no evidence to } \\
\text { support or refute benefits of the } \\
\text { intervention. }\end{array}$ \\
\hline $\begin{array}{l}\text { Satisfaction and acceptability of } \\
\text { treatment - leaving the study early }\end{array}$ & See comment & See comment & See comment & See comment & See comment & $\begin{array}{l}\text { No studies reported this out- } \\
\text { come, so there is no evidence to } \\
\text { support or refute benefits of the } \\
\text { intervention. }\end{array}$ \\
\hline $\begin{array}{l}\text { Mental state (medium term) - total } \\
\text { scores (BPRS, high = poor) } \\
\text { Follow-up: } 6 \text { months }\end{array}$ & $\begin{array}{l}\text { The mean mental } \\
\text { state - average scores } \\
\text { (BPRS, high = poor, } \\
\text { medium term) was } \\
\text { 44.3. }\end{array}$ & $\begin{array}{l}\text { MD } 0.93 \text { low- } \\
\text { er }(6.95 \text { lower } \\
\text { to } 5.09 \text { higher })\end{array}$ & - & $\begin{array}{l}30 \\
\text { (1 study) }\end{array}$ & $\begin{array}{l}\oplus \ominus \ominus \ominus \\
\text { very low } 1,2,3\end{array}$ & $\begin{array}{l}\text { Data for predefined outcome } \\
\text { 'clinically important change' not } \\
\text { reported. }\end{array}$ \\
\hline $\begin{array}{l}\text { General functioning (medium term) - } \\
\text { average scores (GAF, high = good) } \\
\text { Follow-up: } 6 \text { months }\end{array}$ & $\begin{array}{l}\text { The mean general } \\
\text { functioning - average } \\
\text { scores (GAF, high = } \\
\text { good, medium term) } \\
\text { was } \mathbf{3 0 . 1} .\end{array}$ & $\begin{array}{l}\text { MD } 0.66 \text { low- } \\
\text { er ( } 3.6 \text { lower } \\
\text { to } 2.28 \text { higher) }\end{array}$ & - & $\begin{array}{l}30 \\
(1 \text { study })\end{array}$ & $\begin{array}{l}\oplus \oplus \ominus \ominus \\
\text { very low } 1,2,3\end{array}$ & $\begin{array}{l}\text { Data for predefined outcome } \\
\text { 'clinically important change' not } \\
\text { reported. }\end{array}$ \\
\hline Service use - hospitalisation & See comment & See comment & See comment & See comment & See comment & $\begin{array}{l}\text { No studies reported this out- } \\
\text { come, so there is no evidence to } \\
\text { support or refute benefits of the } \\
\text { intervention. }\end{array}$ \\
\hline Adverse event/effect(s) - death & See comment & See comment & See comment & See comment & See comment & $\begin{array}{l}\text { No studies reported this out- } \\
\text { come, so there is no evidence to }\end{array}$ \\
\hline
\end{tabular}

(BPRS, high = poor,

medium term) was

The mean general

scores (GAF, high =

good, medium term)

MD 0.66 low

er (3.6 lower

(1 study)

$\oplus \ominus \ominus \ominus$

'clinically important change' not

. 
${ }^{*}$ The risk in the intervention group (and its $95 \%$ confidence interval) is based on the assumed risk in the comparison group and the relative effect of the intervention (and its $95 \% \mathrm{Cl})$.

BPRS: Brief Psychiatric Rating Scale; CI: confidence interval; ECT: electroconvulsive therapy; GAF: Global Assessment of Functioning; MD: mean difference; RR: risk ratio

GRADE Working Group grades of evidence

High quality: we are very confident that the true effect lies close to that of the estimate of the effect.

Moderate quality: we are moderately confident in the effect estimate; the true effect is likely to be close to the estimate of effect, but there is a possibility that it is substantially different.

Low quality: our confidence in the effect estimate is limited; the true effect may be substantially different from the estimate of the effect.

Very low quality: we have very little confidence in the effect estimate; the true effect is likely to be substantially different from the estimate of effect.

1Downgraded by one level due to risk of bias: Chanpattana 1999 had high risk of bias for blinding of participants and personnel.

2Downgraded by one level due to indirectness: scores from scale were employed as a surrogate index of the intended outcome.

3Downgraded by two levels due to imprecision: small sample size and wide confidence interval. 


\section{B A C K G R O U N D}

\section{Description of the condition}

Schizophrenia is a common mental health condition with an incidence of 15.2 cases per 100,000 per year and a lifetime prevalence of 7.2 cases per 100,000 globally (McGrath 2008). It is a psychotic condition with symptoms that are comprised of fixed beliefs held on unreasonable grounds (delusions), perceptions without a cause (hallucinations), and disorganised thinking (thought disorder). The course of the illness is variable, with a minority of people fully recovering from an initial episode, and most individuals experience a relapsing-remitting course (Harrison 2001). The degree of disability is high, with $80 \%$ to $90 \%$ unemployment (Marwaha 2004). Up to $60 \%$ of people with schizophrenia will respond to treatment, but it is well-recognised that approximately 1 in 3 people have an illness that is 'treatmentresistant' (Meltzer 1997).

Treatment-resistant schizophrenia has not been consistently defined in the literature (Suzuki 2012). In a landmark randomised trial, Kane and colleagues investigated the effects of clozapine compared with chlorpromazine for people with defined treatmentresistant illnesses (Kane 1988). In this study treatment-resistant schizophrenia was defined as "at least three periods of treatment with antipsychotics from at least two different classes at adequate doses for an adequate period time with no relief and no period of good functioning over the previous five years". The criteria used to define treatment resistance in this study remain some of the most cited in randomised trials evaluating people with treatment-resistant schizophrenia (Conley 1997), although there is still heterogeneity in the criteria used.

\section{Description of the intervention}

Electroconvulsive therapy (ECT) was introduced as a treatment for schizophrenia in 1938 as a replacement for chemically induced seizures (Endler 1988). It involves the induction of a seizure ('fit') by administering an electrical stimulus via electrodes usually placed bilaterally on the scalp.

Electroconvulsive therapy can be modified by the use of anaesthetic agents and muscle relaxants to reduce apprehension and the likelihood of adverse outcomes such as spinal and limb fractures that result from the convulsions. In the industrialised world most ECT treatment administered is modified ECT. Unmodified ECT is still used in some parts of the world, especially in areas that do not have ready access to anaesthetic equipment. There is evidence that the use of ECT in less industrialised countries is higher than in higher-income countries (Agarwal 1992; Odejide 1987), and that there is also greater use of unmodified ECT. Possible explanations for this may be that ECT has a perceived greater speed of response and can also be administered inexpensively when compared with drug treatments.

Electroconvulsive therapy has been recognised as an effective treatment for mood disorders, and its use for the treatment of schizophrenia has declined in higher-income countries with most individuals receiving ECT for depressive disorders (Leiknes 2012). Electroconvulsive therapy also pre-dates the introduction of neuroleptic and antidepressant medications by more than a decade, and there is evidence that its use has declined over time (Thompson 1994). This may be due to concerns about long-term side effects such as memory loss (Rose 2003). However, there is no conclusive evidence that ECT results in brain damage (Devanand 1994; Dwork 2004; Ende 2000; Weiner 1986). To reduce the risk of cognitive side effects, ECT is sometimes given unilaterally to the non-dominant hemisphere, although there is evidence that a large dose of electrical stimulus must be used and that the efficacy is less than with bilateral electrode placement (Sackeim 1993; Sackeim 2000a).

There is inconsistency among expert groups about the use of ECT and its indications. The American Psychiatric Association recommends the use of ECT in schizophrenia in the following circumstances: when psychotic symptoms in the present episode have an abrupt or recent onset, when schizophrenia is of the catatonic type, or when there is a history of a favourable response to ECT (APA 2008). The Royal College of Psychiatrists states that 1) while the treatment of choice for schizophrenia is drug therapy, ECT may be considered for treatment-resistant cases and catatonia, and 2) although there is some evidence supporting the shortterm effectiveness of ECT, evidence for sustained medium- and long-term benefits is lacking (Scott 2005). The National Institute for Health and Care Excellence (NICE) only recommends ECT for severe depressive illness, a prolonged or severe episode of mania, or catatonia (which may also occur in other disorders such as depression) (NICE 2003).

Electroconvulsive therapy dosing schedules vary from country to country, with treatments often being delivered three times per week in the USA compared to twice a week in the UK (Scott 2005). There is also considerable variation in practice, with small numbers of clinicians in different parts of the world administering ECT daily or many times a day until a state of regression is achieved (regressive ECT) (Agarwal 1992). The number of sessions in a course typically varies from four to 12 (Weiner 1994). It has been suggested that individuals with schizophrenia may need 12 to 20 sessions (Kendell 1981). Rarely, some patients are also prescribed ECT fortnightly or monthly as 'continuation ECT' or 'maintenance ECT' to prevent relapse (Monroe 1991; Scott 1991). There is evidence that the frequency of ECT influences the speed of response as well as the adverse effects on cognition (Gangadhar 2010).

In addition, there is evidence that the dose of the electrical stimulus also affects the outcome of the treatment, with higher doses of electricity being associated with greater efficacy but more cognitive side effects (Sackeim 1993).

Due to the controversial nature of ECT, there are legal restrictions on its administration when compared with other treatments in many jurisdictions (Kala 2013; Loo 2010). In the UK, for example, a patient who has the capacity to consent to a medication but is refusing it may be administered that medication if they are subject to the Mental Health Act. Comparatively, a patient who has the capacity to consent to ECT but is refusing it may not be administered ECT under the Mental Health Act (Mughal 2013).

The ethics of research in schizophrenia are also debated given that it is a condition that can affect decision making. Individuals with treatment-resistant schizophrenia may be expected to have a severe and enduring illness and considered to be more likely to have impairments in their decision-making capacity. However, there is evidence that, with the appropriate interventions in place, individuals with schizophrenia are able to perform as well as nonill controls in assessments of decisional capacity (Carpenter 2000). 


\section{How the intervention might work}

How ECT works is not well understood. Despite concerns about brain damage and cognitive side effects, there is empirical evidence from animal models that ECT causes neurogenesis (Madsen 2000). Studies from depressed individuals have shown that ECT causes an increase in brain-derived neurotrophic factor (Bocchio-Chiavetto 2006). However, studies have also shown that brain-derived neurotrophic factor may play a potential role in the development of schizophrenia (Muglia 2003; Nieto 2013). Other putative mechanisms include influences on dopamine and serotonin neurotransmitter activity and immune system modulation (Rosenquist 2014).

\section{Why it is important to do this review}

Previous Cochrane Reviews of ECT for the treatment of schizophrenia have not looked specifically at the evidence for ECT in people with treatment-resistant schizophrenia, despite this being a clinical dilemma that is a consensus priority for research (Lloyd 2011; Tharyan 2005). Given that ECT remains a controversial treatment, it is essential to determine its efficacy. This review attempted to assess the current evidence for ECT specifically for the group of people whose illness has been designated as resistant to treatment.

\section{OB JECTIVES}

Our primary objective was to assess the effects (benefits and harms) of ECT for people with treatment-resistant schizophrenia.

Our secondary objectives were to determine whether ECT produces a differential response in people:

- who are treated with unilateral compared with bilateral ECT;

- who have had a long (more than 12 sessions) or a short course of ECT;

- who are given continuation or maintenance ECT;

- who are diagnosed with well-defined treatment-resistant schizophrenia as opposed to less rigorously defined treatmentresistant schizophrenia (who would be expected to have a greater affective component to their illness).

\section{METHODS}

\section{Criteria for considering studies for this review}

\section{Types of studies}

We considered all relevant randomised controlled trials. If a trial was described as 'double blind' but implied randomisation, we planned to include such trials in a sensitivity analysis (Sensitivity analysis). We excluded quasi-randomised studies such as those allocating by alternate days of the week. For cases in which people were given additional treatments to ECT, we only included data if the adjunct treatment was evenly distributed between the groups, and only the ECT was randomised.

\section{Types of participants}

We included people of both sexes, aged 18 years or more, with a diagnosis of treatment-resistant schizophrenia or related disorders (e.g. schizoaffective disorder, schizophreniform disorder), regardless of how it was diagnosed. We planned to conduct a sensitivity analysis by only including people with strictly diagnosed schizophrenia by international standards (International Statistical Classification of Diseases and Related Health Problems 10th Revision (ICD-10), Diagnostic and Statistical Manual of Mental Disorders (DSM) (DSM-III, DSM-IV, DSM-5), Chinese Classification of Mental Disorders (CCMD) (CCMD-1, CCMD-2, CCMD-3).

\section{Types of interventions}

\section{ECT}

Electroconvulsive therapy is a procedure that involves passing an electrical stimulus through the brain to produce a seizure. It is a practice in many countries to administer 'modified' ECT, which entails administering a general anaesthetic and muscle relaxant as well as the electrical stimulus. Electroconvulsive therapy can normally be delivered as a course over several sessions. We did not exclude studies based on the number and frequency of ECT sessions or whether the ECT was modified or unmodified. We also did not exclude studies in which the participants were receiving concurrent pharmacological interventions and other interventions, provided that only ECT was being randomised.

\section{Standard care}

For this review we have defined standard care as the treatment the participants received alongside the trial intervention as part of their ongoing care for their illness.

We planned to compare ECT therapy with the following.

\section{'Sham-ECT' or 'simulated-ECT'}

'Sham-ECT' or 'simulated-ECT' is a situation in which a patient undergoes all the preparations for ECT, often including receiving a general anaesthetic and muscle relaxant, but does not receive the electrical stimulus.

\section{Treatment with antipsychotics}

Antipsychotic drugs are medications designed to treat psychosis; they are thought to act in part by their dopamine-blocking action, although other chemical pathways have also been identified.

\section{Non-pharmacological forms of treatment}

Non-pharmacological management of schizophrenia includes but is not limited to interventions such as occupational therapy and psychotherapy.

\section{Placebo}

Any intervention that is not thought to be an active treatment for schizophrenia.

\section{Standard care}

For this review we have defined standard care as the treatment the participants received alongside the trial intervention as part of their ongoing care for their illness.

\section{ECT alone}

Electroconvulsive therapy may be the sole treatment that is prescribed for a patient. For this comparison, we excluded studies in which the participants were prescribed concurrent interventions, whether pharmacological or non-pharmacological, except when these interventions were part of the process of ECT. We also planned to include studies in which participants received alternative interventions, provided these studies were randomised 
comparisons with ECT. We did not identify any study of this type in the review.

We planned to compare ECT alone with the following:

- sham-ECT or simulated-ECT;

- treatment with antipsychotics;

- non-pharmacological forms of treatment;

- placebo

- standard care

However, we did not identify any relevant studies comparing ECT alone with the following therapies: sham-ECT or simulated-ECT, non-pharmacological forms of treatment, placebo or standard care

\section{Electrode placement}

\section{Bilateral ECT versus unilateral ECT}

In bilateral ECT, the electrical stimulus is administered across both hemispheres of the brain. The placement is usually bi-temporal. In unilateral ECT, the electrical stimulus is administered to the non-dominant hemisphere of the brain, and the placement is usually temporoparietal. We did not identify any studies with this comparison for inclusion in the review.

\section{Duration of course}

\section{Course of 6 to 12 ECT sessions versus course of 12 to 30 sessions}

A session of ECT is a session in which an electrical stimulus is delivered to the brain. A repeat stimulus may be administered in the same session if the seizure was inadequate. A typical course of ECT for depression is 6 to 12 sessions, but this is not well-defined for schizophrenia. We planned to determine if there was evidence that people with schizophrenia required a longer course of ECT than that given for people with depression. We did not identify any studies with this comparison for inclusion in the review.

\section{Frequency of treatment}

ECT administered fortnightly or monthly versus any other treatment

Electroconvulsive therapy is normally administered at least weekly. In some situations, it is given less often and may be called 'maintenance' ECT. We defined 'maintenance' ECT as ECT that is delivered either fortnightly or monthly for at least six sessions. We planned to compare maintenance ECT with any other pharmacological or non-pharmacological treatment strategies. We did not identify any studies with this comparison for inclusion in the review.

\section{Types of outcome measures}

If possible, we divided all outcomes into short term (less than six weeks), medium term (six weeks to six months), and long term (over six months).

\section{Primary outcomes}

\section{Response to treatment}

Clinically important response to treatment - as defined by the original studies

\section{Cognitive functioning}

Clinically important change in cognitive functioning - as defined by individual studies

\section{Secondary outcomes}

\section{Satisfaction and acceptability of treatment}

1.1. Leaving the study early

1.2. Participants reporting adverse events, e.g. post-ECT headache

\section{Mental state}

2.1. Clinically important change in general mental state at short, medium, and long term

2.2. Average endpoint general mental state score

2.3. Average change in general mental state scores

2.4. Clinically important change in specific symptoms (positive symptoms of schizophrenia, negative symptoms of schizophrenia) at short, medium, and long term

2.5. Average endpoint specific symptom score

2.6. Average change in specific symptom scores

\section{General functioning}

3.1. Clinically important change in general functioning at short and medium term

3.2. Average endpoint general functioning score

3.3. Average change in general functioning scores

\section{Service use}

4.1. Number hospitalised

4.2. Number discharged or readmitted (as defined in individual trials)

\section{Adverse event/effect(s)}

\subsection{General}

5.1.1. Any major adverse event

5.1.2. Any reported adverse event

5.1.3. Average endpoint in general adverse event score

5.1.4. Average change in general adverse event score

\subsection{Specific}

5.2.1. Any major adverse event

5.2.2. Any reported adverse event

5.2.3. Average endpoint in specific adverse event score (e.g. cognitive function)

5.2.4. Average change in specific adverse event score 5.2.5. Death

\section{'Summary of findings' table}

We used GRADEpro GDT to import data from Cochrane's statistical software Review Manager 5 to create the 'Summary of findings' tables (Review Manager). These tables provide outcome-specific information concerning the overall quality of the evidence from each included study in the comparison, the magnitude of the effect of the interventions examined, and the sum of available data on all outcomes that we rated as important to patient care and decision making.

We selected the following main outcomes for inclusion in the 'Summary of findings' table. 
- Response to treatment: clinically important response - as defined by each of the studies.

- Cognitive functioning: clinically important change in cognitive functioning - as defined by each of the studies.

- Acceptability of treatment: leaving the study early.

- Mental state: clinically important change in general mental state - as defined by each of the studies.

- General functioning: clinically important change in general functioning - as defined by each of the studies.

- Service use: number hospitalised

- Adverse events/effects: death.

\section{Search methods for identification of studies}

\section{Electronic searches}

\section{Cochrane Schizophrenia Group's Study-Based Register of Trials}

On 9 September 2015 and 4 August 2017, the Cochrane Schizophrenia Group Information Specialist searched the register using the following search strategy:

- ("Electroconvulsive Therapy ${ }^{\star}$ in Intervention Field) AND ( ${ }^{\star}$ Treatment Resistant* in Healthcare Condition Field) of STUDY

In such a study-based register, searching the major concept retrieves all the synonyms and relevant studies because all the studies have already been organised based on their interventions and have been linked to the relevant topics.

This register was compiled by systematic searches of major resources (including AMED (Allied and Complementary Medicine), BIOSIS, CINAHL (Cumulative Index to Nursing and Allied Health Literature), Embase, MEDLINE, PsycINFO, PubMed, and registries of clinical trials) and their monthly updates, handsearches, grey literature, and conference proceedings (see Group's Module). There were no limitations on language, date, document type, or publication status for the inclusion of records into the register.

\section{Searching other resources}

We inspected the references of all included records to identify further relevant studies. Contact information was available for five included studies (Jiang 2009; Petrides 2015; Wang 2008; Wang 2011; Zhang 2010). We contacted the first author of these studies for information regarding unpublished trials. We noted the outcome of this contact in the 'Characteristics of included studies' or 'Characteristics of studies awaiting classification' tables of the review.

\section{Data collection and analysis}

\section{Selection of studies}

Two review authors (DS, FQ) independently inspected all of the citations from the searches and identified relevant abstracts. When disputes arose, we retrieved the full-text report for further assessment. We obtained the full reports of the records that met the review criteria, and the two review authors (DS, FQ) independently inspected these. In cases of disagreement, we consulted a third review author (SZ). Where doubt still remained, we added these trials to the list of those studies awaiting classification and attempted to contact the study authors for clarification.

\section{Data extraction and management}

\section{Extraction}

Two review authors (DS, FQ) independently extracted data from all the included studies. Again, any disagreements were discussed, decisions documented, and, if necessary, attempts made to contact the authors of the studies for clarification. If there were any remaining issues, we consulted a third review author (SZ) for clarification and documented these final decisions. We extracted data presented only in graphs and figures whenever possible, but only included data for which the two review authors had independently extracted the same results. We attempted to contact authors through an open-ended request in order to obtain missing information or for clarification whenever necessary. For multicentre studies, we planned whenever possible to extract data relevant to each component centre separately. However, data were not available for each centre in the relevant studies.

\section{Management}

\subsection{Data collection forms}

We extracted data onto pre-standardised data extraction forms.

\subsection{Scale-derived data}

We included continuous data from rating scales only if:

- the psychometric properties of the measuring instrument had been described in a peer-reviewed journal (Marshall 2000); and

- the measuring instrument had not been written or modified by one of the trialists for that particular trial.

Ideally, the measuring instrument should either be a self report tool or one that was completed by an independent rater or a relative (not the therapist). We realise that this is often not reported clearly, therefore we made a note of the description of the measuring instrument in the Description of studies section.

\subsection{Endpoint versus change data}

There are advantages to both endpoint and change data. Change data can remove a component of between-person variability from the analysis. On the other hand, a calculation of change needs two assessments (baseline and endpoint), which can be difficult in unstable and hard-to-measure conditions such as schizophrenia. We decided to primarily use endpoint data and to only use change data if the former were not available. We planned to combine endpoint and change data in the analysis because we preferred to use mean differences (MDs) rather than standardised mean differences (SMDs) throughout (Higgins 2011). Ultimately, the endpoint data were available in all the included studies.

\subsection{Skewed data}

Continuous data on clinical and social outcomes are often not normally distributed. To avoid the pitfall of applying parametric tests to non-parametric data, we applied the following standards to all the data before inclusion.

- For change data, we planned to enter relevant useable change data into the analyses. For instance, when continuous data are presented on a scale that includes a possibility of negative values (such as change data), it is difficult to tell whether the data are skewed or not. However, no change data were identified. 
- For endpoint data from small trials $(n<200)$ :

* when a scale started from the finite number 0 , we subtracted the lowest possible value from the mean, and divided this by the standard deviation (SD). If this value was lower than 1 , it strongly suggested a skew, and we excluded the study data. If this ratio was higher than 1 but below 2, there was a suggestion of skew. We entered the study data and tested whether inclusion or exclusion changed the results substantially. Finally, if the ratio was larger than 2, we included the study data, because a skew was less likely (Altman 1996; Higgins 2011);

* if a scale started from a positive value (such as the Positive and Negative Syndrome Scale (PANSS), which has values from 30 to 210) (Kay 1986), we modified the calculation described above to take into account the scale starting point. In such cases, a skew was present if $2 \mathrm{SD}>$ (S - S min), where $\mathrm{S}$ was the mean score and $\mathrm{S}$ min was the minimum score.

- For endpoint data from larger trials ( $n>200$ ), we entered the relevant endpoint data from studies of at least 200 participants in the analyses irrespective of the above rules because skewed data poses less of a problem in large studies.

\subsection{Common measures}

To facilitate comparisons between trials, we planned to convert variables that could be reported in different metrics, such as days in hospital (mean days per year, per week, or per month) to a common metric (e.g. mean days per month).

\subsection{Conversion of continuous to binary data}

Whenever possible, we made efforts to convert outcome measures to dichotomous data. This could be done by identifying cut-off points in rating scales and dividing participants accordingly into 'clinically improved' or 'not clinically improved'. It was generally assumed that if there was a $50 \%$ reduction in a scale-derived score such as the Brief Psychiatric Rating Scale (BPRS) or the PANSS (Kay 1986; Overall 1962), this could be considered a clinically significant response (Leucht 2005; Leucht 2005a). If data based on these thresholds were not available, we used the primary cut-off presented by the original authors.

\subsection{Direction of graphs}

Whenever possible, we entered data in such a way that the area to the left of the line of no effect indicated a favourable outcome for ECT. In cases where keeping to this made it impossible to avoid outcome titles with clumsy double-negatives (e.g. 'Not unimproved'), we reported data where the left of the line indicated an unfavourable outcome and noted this in the relevant graphs.

\section{Assessment of risk of bias in included studies}

Two review authors (DS, FQ) independently assessed risk of bias using the criteria described in the Cochrane Handbook for Systematic Reviews of Interventions (Higgins 2011). This set of criteria is based on evidence of associations between an overestimate of effect and a high risk of bias for the article related to sequence generation, allocation concealment, blinding, incomplete outcome data, and selective reporting.

In case of disagreement, the final rating was made by consensus with the involvement of a third review author (SZ). When inadequate details of randomisation and other characteristics of trials were provided, we attempted to contact the authors of the studies to obtain further information. However, we did not receive any reply from the authors we contacted. We reported nonconcurrence in the quality assessment, but if disputes arose as to which category a trial should be allocated to, we again resolved the issue by discussion.

We noted the level of risk of bias in both the text of the review and in the 'Summary of findings' table.

\section{Measures of treatment effect}

\section{Binary data}

For binary outcomes, we calculated the standard estimation of risk ratios (RRs) and their 95\% confidence intervals (Cls). It has been shown that RRs are more intuitive than odds ratios (ORs), and that ORs tend to be interpreted as RRs by clinicians (Boissel 1999; Deeks 2000). The number needed to treat for an additional beneficial outcome/harmful outcome (NNTB/H) statistics with $95 \% \mathrm{Cls}$ are intuitively attractive to clinicians but can be problematic both in terms of accurate calculation in meta-analyses and interpretation (Hutton 2009). For binary data presented in the 'Summary of findings' tables, we calculated illustrative comparative risks where possible.

\section{Continuous data}

For continuous outcomes, we estimated MDs between the groups. We preferred not to calculate measures of effect size (i.e. using SMDs). However, if scales of very considerable similarity were used, we planned to presume there was a small difference in measurement. We also planned to calculate effect size and transform the effect back to the units of one or more of the specific instruments. We reported the outcomes measured by different scales separately in the review.

\section{Unit of analysis issues}

\section{Cluster trials}

Though studies increasingly employ 'cluster randomisation' (such as randomisation by clinician or practice), the analysis and pooling of clustered data pose problems. First, authors often fail to account for intraclass correlations in clustered studies, leading to a 'unit of analysis' error (Divine 1992), whereby P values are spuriously low, $\mathrm{Cls}$ unduly narrow, and statistical significance overestimated. This causes type I errors (Bland 1997; Gulliford 1999).

For cases where clustering was not accounted for in the primary studies, we planned to present data in a table, with a $\left(^{*}\right)$ symbol to indicate the presence of a probable unit of analysis error. We planned to attempt to contact the first authors of these studies to obtain intraclass correlation coefficients (ICCs) for their clustered data and adjust for this by using accepted methods (Gulliford 1999). For cases where clustering had been incorporated into the analysis of primary studies, we planned to present these data as if from a non-cluster-randomised study, but adjust for the clustering effect.

We sought statistical advice and were advised that the binary data as presented in a report should be divided by a 'design effect'. This is calculated using the mean number of participants per cluster $(\mathrm{m})$ and the ICC (design effect $=1+(m-1)^{\star} I C C$ ) (Donner 2002). If the ICC was not reported, we would assume it to be 0.1 (Ukoumunne 1999).

If cluster studies were appropriately analysed taking into account ICCS and relevant data documented in the report, synthesis with 
other studies would be possible using the generic inverse-variance technique.

However, we did not identify any study with a cluster-randomised design for inclusion in the review.

\section{Cross-over trials}

A major concern of cross-over trials is the carry-over effect. This occurs if an effect (e.g. pharmacological, physiological, or psychological) of the treatment in the first phase is carried over to the second phase. As a consequence, upon entry to the second phase participants can differ systematically from their initial state despite a wash-out phase. For the same reason cross-over trials are not appropriate if the condition of interest is unstable (Elbourne 2002). As both effects are very likely in cases of severe mental illness, we planned to only use data from the first phase of crossover studies. However, we did not identify any study with a crossover design for inclusion in the review.

\section{Studies with multiple treatment groups}

When a study involved more than two treatment arms, if relevant, we presented the additional treatment arms in the comparisons. For binary data, we planned to simply add them together and combine them within the two-by-two table. For continuous data, we planned to combine data following the formula in the Cochrane Handbook for Systematic Reviews of Interventions (Higgins 2011). When the additional treatment arms were not relevant, we planned not to use these data.

\section{Dealing with missing data}

\section{Overall loss of credibility}

At some degree of loss of follow-up data there is a loss of credibility (Xia 2009). If for any particular outcome more than $50 \%$ of the data were unaccounted for, we planned not to reproduce these data or use them in the analyses. If, however, more than $50 \%$ of the data in one arm of a study were lost, but the total loss was less than $50 \%$, we planned to address this within the 'Summary of findings' tables by down-rating quality. Finally, we also planned to downgrade quality within the 'Summary of findings' tables should the loss of data be between $25 \%$ and $50 \%$ in total. However, we did not identify any studies with the above-stated issues for inclusion in the review.

\section{Binary}

For cases where attrition for a binary outcome was between 0 and $50 \%$, and these data were not clearly described, we presented data on a 'once-randomised-always-analyse' basis (an intentionto-treat (ITT) analysis). We assumed that all participants who left the study early had the same rate of negative outcomes as those who completed the study, with the exception of the outcomes of death and adverse effects. For these outcomes, the rate of negative outcomes for those who stayed in the study - in that particular arm of the trial - was used for those who left the study. We undertook a sensitivity analysis to test how prone the primary outcomes were to change when data from only people who completed the study up to that point were compared with the ITT analysis using the above assumptions.

\section{Continuous}

\subsection{Attrition}

For cases where attrition for a continuous outcome was between 0 and $50 \%$, and data from only people who completed the study up to that point were reported, we used these data.

\subsection{Standard deviations}

If standard deviations (SDs) were not reported, we first planned to attempt to obtain the missing values from the authors. If this information was not available, when there were missing measures of variance for continuous data, but an exact standard error (SE) and $\mathrm{Cls}$ were available for group means, and either $\mathrm{P}$ or $\mathrm{T}$ values were available for differences in the mean, we planned to calculate them according to the rules described in the Cochrane Handbook for Systematic Reviews of Interventions (Higgins 2011): when only the SE was reported, we planned to calculate SDs using the formula $\mathrm{SD}=\mathrm{SE}$ * square root (n). The Cochrane Handbook for Systematic Reviews of Interventions provides detailed formulae for estimating SDs from $\mathrm{P}$, T, or F values, Cls, ranges, or other statistics (Higgins 2011). If these formulae did not apply, we planned to calculate the SDs according to a validated imputation method based on the SDs of the other included studies (Furukawa 2006). Although some of these imputation strategies can introduce error, the alternative would be to exclude a given study's outcome and thus lose information. We nevertheless planned to examine the validity of the imputations in a sensitivity analysis excluding the imputed values. However, we did not perform any imputations on missing SDs in this review.

\subsection{Assumptions about participants who left the trials early or were lost to follow-up}

Various methods are available to account for participants who leave the trials early or are lost to follow-up. Some trials just present the results of study completers, while others use the method of 'last observation carried forward' (LOCF). More recently methods such as multiple imputation or mixed-effects models for repeated measurements have become more of a standard. While the latter methods seem to be somewhat better than LOCF (Leon 2006), we felt that the high percentage of participants leaving the studies early and the differences in their reasons for leaving early between groups were often the core problem in randomised trials evaluating schizophrenia. We therefore did not exclude studies based on the statistical approach used. However, we preferred to use the more sophisticated approaches, for example mixed-effects models for repeated measurements or multiple imputation instead of LOCF. In addition, we only presented completer analyses if some kind of ITT data were completely unavailable. Moreover, we addressed this issue in the incomplete outcome data domain of the 'Risk of bias' tool.

\section{Assessment of heterogeneity}

\section{Clinical heterogeneity}

To judge clinical heterogeneity, we considered all the included studies initially without looking at the comparison data. We simply inspected all studies for clearly outlying people or situations that we had not predicted would arise. When such situations or participant groups arose, we discussed these in detail. 


\section{Methodological heterogeneity}

To judge methodological heterogeneity, we considered all the included studies initially without looking at the comparison data. We simply inspected all studies for clearly outlying methods that we had not predicted would arise. When such methodological outliers arose, we discussed these in detail.

\section{Statistical heterogeneity}

\subsection{Visual inspection}

We visually inspected graphs to investigate the possibility of statistical heterogeneity.

\subsection{Employing the $\left.\right|^{2}$ statistic}

We investigated statistical heterogeneity between studies by considering the $\mathrm{I}^{2}$ statistic alongside the $\mathrm{P}$ value of the $\mathrm{Chi}^{2}$ test. The $1^{2}$ statistic provides an estimate of the percentage of inconsistency thought to be due to chance (Higgins 2003). The importance of the observed value of $\mathrm{I}^{2}$ depends on both the magnitude and direction of the effects and the strength of evidence for heterogeneity (e.g. $\mathrm{P}$ value from $\mathrm{Chi}^{2}$ test or $\mathrm{Cls}$ for $\mathrm{I}^{2}$ ). We considered an $\mathrm{I}^{2}$ statistic estimate of greater than or equal to approximately $50 \%$ accompanied by a statistically significant $\mathrm{Chi}^{2}$ test $(\mathrm{P}<0.1)$ as evidence of substantial levels of heterogeneity (Higgins 2011). When we found substantial levels of heterogeneity, we explored reasons for the heterogeneity (Subgroup analysis and investigation of heterogeneity).

\section{Assessment of reporting biases}

\section{Protocol versus full study}

Reporting biases arise when the dissemination of research findings is influenced by the nature and direction of the results (Higgins 2011). We attempted to locate the protocols of the included randomised trials. We planned that if the protocol was available, we would compare the outcomes in the protocol and with those in the published report. If the protocol was not available, we compared the outcomes listed in the methods section of the trial report with the actual reported results.

\section{Funnel plots}

Reporting biases arise when the dissemination of research findings is influenced by the nature and direction of the results (Egger 1997; Higgins 2011). We are aware that funnel plots may be useful in investigating reporting biases, but are of limited power to detect small-study effects. We did not use funnel plots for outcomes when there were 10 or fewer studies, or when all studies were of a similar size. In other cases, when funnel plots were possible, we planned to seek statistical advice for their interpretation. However, there were no outcomes for which there were more than 10 studies.

\section{Data synthesis}

We understand that there is no definitive argument that supports a preference for use of the fixed-effect or the random-effects model. The random-effects method incorporates an assumption that the different studies are estimating different yet related intervention effects. This seems often to be true to us. Furthermore, the randomeffects model takes into account differences between studies even if there is no statistically significant heterogeneity. There is, however, a disadvantage to the random-effects model, in that it gives added weight to small studies, which are often the most biased. Depending on the direction of the effect, these studies can either inflate or deflate the effect size. We chose the fixed-effect model for all analyses. However, the reader can choose to inspect the data using the random-effects model.

\section{Subgroup analysis and investigation of heterogeneity}

\section{Secondary objectives}

Our secondary objectives were to determine whether ECT produced a differential response in the following subgroups:

- people who were treated with unilateral compared with bilateral ECT;

- people who had a long (more than 12 months) versus a short course of ECT;

- people who were given continuation or maintenance ECT;

- people who were diagnosed with well-defined treatmentresistant schizophrenia as opposed to those with less rigorously defined treatment-resistant schizophrenia.

If the trials directly compared the techniques (e.g. people were randomised to unilateral or bilateral ECT), then we would present these data within the relevant comparison. We did not identify any study that directly compared techniques for inclusion in the review. However, if, within a comparison, data were reported on subgroups of people (e.g. within the unilateral-versus-bilateral comparison, data were presented for those who had a long course compared with those who had a shorter course), then we reported these subgroups. We did this only for the primary outcomes.

\section{Investigation of heterogeneity}

If data were clearly heterogeneous, we checked that they had been extracted and entered correctly, and that no unit of analysis errors had occurred. If high levels of heterogeneity remained, we did not undertake a meta-analysis at this point, as if there is considerable variation in results, and particularly inconsistency in the direction of effect, it may be misleading to quote an average value for the intervention effect.

When unanticipated clinical or methodological heterogeneity was obvious, we would simply state hypotheses regarding this for future reviews or versions of this review. We did not anticipate undertaking future analyses related to this.

\section{Sensitivity analysis}

\section{Implication of randomisation}

We planned to include trials in a sensitivity analysis if they were described in a way that implied randomisation. For the primary outcomes, we planned to include these studies. If there was no substantive difference when the implied randomised studies were added to those with a better description of randomisation, then we would use any relevant data from these studies. However, we did not identify any study with the above-stated issues for inclusion in the review.

\section{Assumptions for lost binary data}

For cases where assumptions had to be made regarding people lost to follow-up (see Dealing with missing data), we compared the findings of the primary outcomes when we used our assumptions 
and when we used data only from people who completed the study up to that point. If there was a substantial difference, we would report the results and discuss them but would continue to use our assumption.

For cases where assumptions had to be made regarding missing SDs (see Dealing with missing data), we planned to compare the findings of the primary outcomes when we used our assumptions and when we used data only from people who had completed the study up to that point. We planned to undertake a sensitivity analysis to test how prone the results were to change when only completer-only data were compared with the imputed data using the above assumption. If there was a substantial difference, we would report results and discuss them, but would continue to use our assumption. However, we did not make any assumptions regarding missing SDs.

\section{Risk of bias}

For the meta-analysis of the primary outcome, we planned to analyse the effects of excluding trials that were judged to be at high risk of bias across one or more of the domains of randomisation (implied as randomised with no further details available) including allocation concealment, blinding, and outcome reporting. If the exclusion of trials at high risk of bias did not substantially alter the direction of the effect or the precision of the effect estimates, then we would include data from these trials in the analysis. This sensitivity analysis was not available because all the included studies were at high risk of bias in at least one domain.

\section{Imputed values}

We also planned to undertake a sensitivity analysis to assess the effects of including data from trials for which we had used imputed values for ICC to calculate the design effect in cluster-randomised trials.

If we noted substantial differences in the direction or precision of effect estimates in any of the sensitivity analyses listed above, we would not pool data from the excluded trials with the other trials contributing to the outcome, but would present them separately.
However, we did not identify any studies with a cluster-randomised design for inclusion in the review.

\section{Fixed-effect versus random-effects model}

We analysed all the data using a fixed-effect model; however, we also synthesised data for the primary outcome using a randomeffects model to evaluate whether this altered the significance of the result.

\section{Diagnostic criteria}

We planned to undertake a sensitivity analysis to assess the effects of excluding trials in which participants did not have strictly diagnosed schizophrenia by international standards (ICD-10, DSMIII, DSM-IV, DSM-5, CCMD-1, CCMD-2, or CCMD-3). If there was a substantial difference in our results, then we would report these differences and discuss them. The sensitivity analysis was not available because all included studies enrolled participants with strictly diagnosed schizophrenia by international standards.

\section{RESULTS}

\section{Description of studies}

See Characteristics of included studies; Characteristics of excluded studies; Characteristics of studies awaiting classification; Characteristics of ongoing studies.

\section{Results of the search}

The initial search resulted in a total of 255 references that were identified from databases. We identified no additional references through other sources. After de-duplication, 252 unique references remained. We excluded an additional 154 references upon inspection of the titles and abstracts. We read the remaining 73 studies (with 98 references) in full and subsequently excluded 49 studies (with 65 references) with reasons (further details are provided in Figure 1). Six studies (with 8 references) are awaiting assessment, and three studies are ongoing. We included 15 studies (with 22 references) in the review. 


\section{Figure 1. Study flow diagram.}

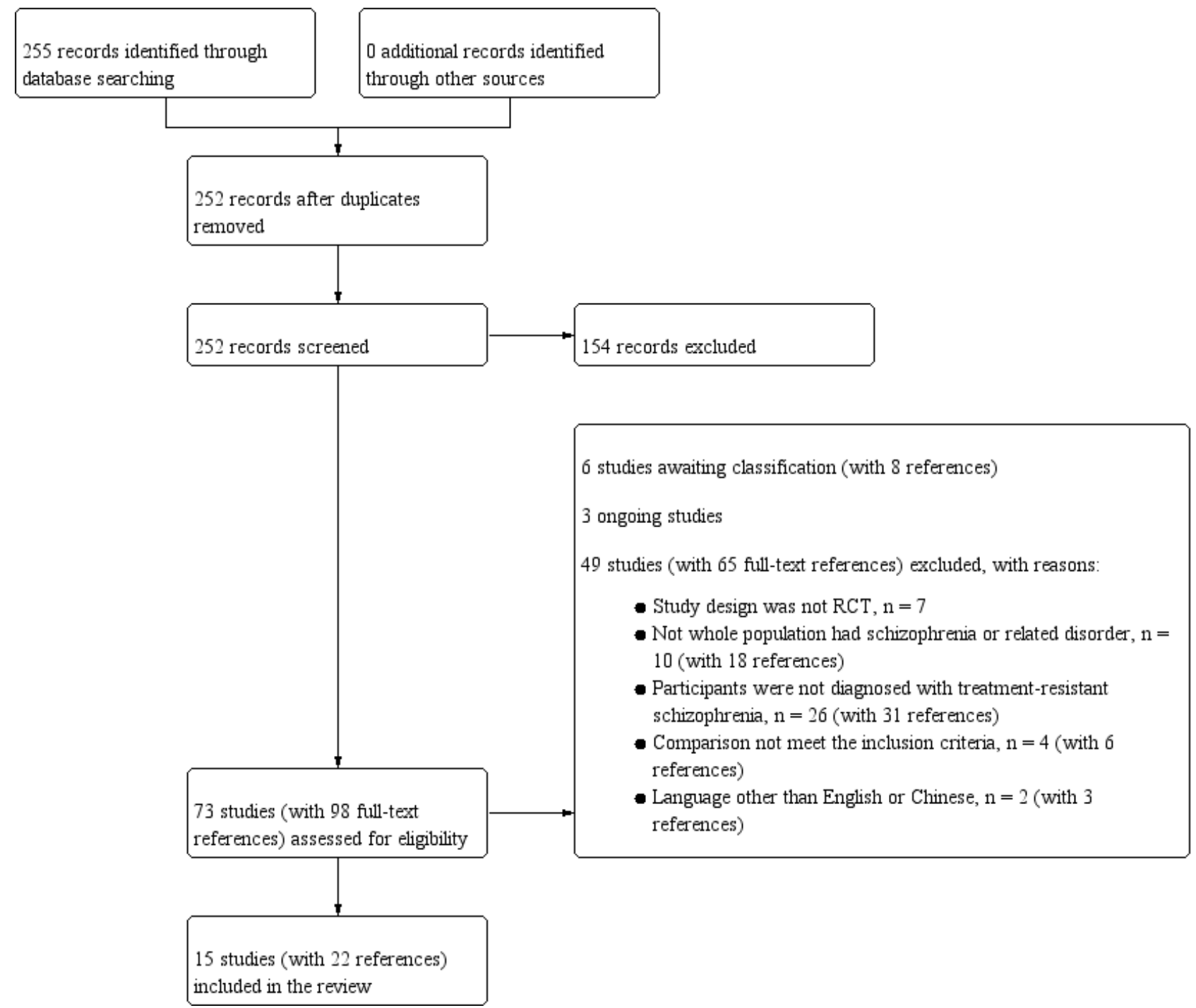

\section{Included studies}

A total of 15 randomised controlled trials involving 1285 participants (1264 completers) met the inclusion criteria for this review. The sample sizes ranged from 31 to 246 (see Characteristics of included studies).

\section{Participants}

Most of the participants $(n=1164)$ were recruited from China (Cai 2008; Chen 2012; Jiang 2009; Jiang 2013; Lin 2014; Liu 2010; Wang 2008; Wang 2011; Wang 2013; Yang 2005; Zhang 2010; Zhang 2012). The remaining participants were recruited from India $(n=31$; Goswami 2003), Thailand ( $n=51$; Chanpattana 1999), and the USA ( $n=39$; Petrides 2015).

The age of the included participants ranged from 18 to 46 years; there were 704 males and 567 females. Three studies did not report the age and sex of the 14 participants who left early (Chanpattana 1999; Goswami 2003; Jiang 2009). The included participants were all diagnosed with treatment-resistant schizophrenia by international standards, including CCMD-2-R, CCMD-3, DSM-IV, and
ICD-10. The average length of illness ranged from 6.3 to 18.6 years, while four studies $(n=287$ ) did not report the average length of the illness (Cai 2008; Liu 2010; Petrides 2015; Wang 2008).

\section{Interventions}

In all 15 studies participants in the ECT group were given ECT or modified ECT (MECT) in addition to standard care (where standard care was an active intervention such as an antipsychotic given to all participants in both treatment groups). One of the studies, Chanpattana 1999, had three treatment arms where in addition to the ECT arm, the study also had another treatment arm $(n=15)$ where ECT was given alone. Five studies $(n=439)$ reported the use of bilateral electrode placement (Chanpattana 1999; Goswami 2003; Petrides 2015; Wang 2013; Zhang 2010), while the placement was unclear in the remaining studies. Ten studies $(n=787)$ used a short course (6 to 12 ECT sessions) of ECT (Cai 2008; Chen 2012; Goswami 2003; Jiang 2009; Jiang 2013; Liu 2010; Wang 2008; Wang 2011; Wang 2013; Yang 2005); four studies $(n=420)$ used a long course (14 to 20 sessions) (Chanpattana 1999; Petrides 2015; Zhang 2010; Zhang 2012), while Lin 2014 ( $n=78$ ) did not report the course of ECT. Five studies $(n=335)$ used ECT three times a week (Cai 
2008; Goswami 2003; Liu 2010; Wang 2013; Yang 2005), and one study $(n=76)$ used ECT once every other day (Wang 2008). Eight studies $(n=796)$ did not use ECT with a unique frequency within the treatment period (Chanpattana 1999; Chen 2012; Jiang 2009; Jiang 2013; Petrides 2015; Wang 2011; Zhang 2010; Zhang 2012); of these, most studies used ECT three times a week for two to four weeks, then once or twice a week for the subsequent weeks. The remaining study $(n=78)$ did not report information on frequency (Lin 2014).

The included studies did not cover all predefined comparisons in our protocol; only the following comparisons were involved:

- ECT plus standard care versus sham-ECT plus standard care (one study, Goswami 2003; chlorpromazine used as the standard care);

- ECT plus standard care versus clozapine plus standard care (one study, Jiang 2013; ziprasidone used as the standard care);

- ECT plus standard care versus standard care (13 studies, Cai 2008; Chanpattana 1999; Chen 2012; Jiang 2009; Lin 2014; Liu 2010; Petrides 2015; Wang 2008; Wang 2011; Wang 2013; Yang 2005; Zhang 2010; Zhang 2012; various antipsychotics used as the standard care across treatment groups, including clozapine, olanzapine, risperidone, chlorpromazine, perphenazine, flupenthixol, and ziprasidone);

- ECT alone versus flupenthixol alone (one study, Chanpattana 1999).

The treatment duration of the included studies ranged from 2 weeks, in Goswami 2003, to 24 weeks, in Chanpattana 1999. The treatment duration in the other studies was 4 weeks (Liu 2010; Wang 2013), 8 weeks (Cai 2008; Jiang 2013; Lin 2014; Petrides 2015; Yang 2005; Zhang 2010; Zhang 2012), or 12 weeks (Chen 2012; Jiang 2009; Wang 2008; Wang 2011).

\section{Primary outcomes}

\section{Response to treatment}

Eleven studies reported the response to treatment. One study reported the short-term outcome (Wang 2013), while the other studies reported the medium-term outcome (Cai 2008; Chen 2012; Jiang 2009; Jiang 2013; Petrides 2015; Wang 2008; Wang 2011; Yang 2005; Zhang 2010; Zhang 2012). The clinically significant response in all studies (except for Petrides 2015) was defined as a 50\% reduction in the BPRS or PANSS scores. The response criterion in Petrides 2015 was defined as $\geq 40 \%$ improvement based on the psychotic symptom sub scale (PSYRATS), a Clinical Global Impression (CGI)-severity rating of mild or less $(<3)$, and a CGIimprovement rating of much improved $(\leq 2)$.

\section{Cognitive functioning}

No study reported a clinically important change in participants' cognitive functioning. One study reported the incidence of shortterm memory deterioration (Wang 2013). Two studies reported the average endpoint cognitive functioning scores (Jiang 2009; Zhang 2010). The following scales were used in this review.

- Wechsler Memory Scale (WMS): a neuropsychological test designed to measure different memory functions. Higher scores indicate better memory.

- Wisconsin Card Sorting Test (WCST): a neuropsychological test of the ability to display flexibility in the face of changing schedules of reinforcement.

\section{Secondary outcomes}

\section{Satisfaction and acceptability of treatment}

Three studies reported the number of participants who left the study early (Jiang 2009; Petrides 2015; Zhang 2010).

\section{Mental state}

No study reported a clinically important change in participants' general mental state. Twelve studies reported the average endpoint general mental state scores (Cai 2008; Chanpattana 1999; Chen 2012; Goswami 2003; Jiang 2009; Jiang 2013; Petrides 2015; Wang 2008; Wang 2011; Yang 2005; Zhang 2010; Zhang 2012), and 13 studies reported average endpoint specific symptom scores (Cai 2008; Chen 2012; Jiang 2009; Jiang 2013; Lin 2014; Liu 2010; Petrides 2015; Wang 2008; Wang 2011; Yang 2005; Zhang 2010; Zhang 2012). The following scales were used in this review.

- Brief Psychiatric Rating Scale (BPRS): an 18-item scale measuring positive symptoms, general psychopathology, and affective symptoms. Each item is rated on a 7-point scale ranging from 'not present' to 'extremely severe'. The possible scores range from 18 to 126 , with high scores indicating more severe symptoms.

- Mini-Mental State Examination (MMSE): a 30-point questionnaire that is used extensively in clinical and research settings to measure cognitive impairment. Lower scores indicate more severe cognitive impairment.

- Positive and Negative Syndrome Scale (PANSS): a 30-item scale including three sub scales for measuring the severity of positive symptoms, negative symptoms, and general psychopathology. Each item is rated on a 7-point scale. The possible scores range from 21 to 210, with higher scores indicating a worse outcome.

- Scale for the Assessment of Negative Symptoms (SANS): a valid instrument to assess the negative symptoms of schizophrenia. Each item is based on a 6-point scale. Higher scores indicate more severe symptoms.

- Scale for Assessment of Positive Symptoms (SAPS): a valid instrument to assess the positive symptoms of schizophrenia. Each item is based on a 6-point scale. Higher scores indicate more severe symptoms.

\section{General functioning}

No study reported a clinically important change in participants' general functioning. Two studies reported the average endpoint general functioning scores assessed by the Global Assessment of Functioning (GAF) scale (Chanpattana 1999; Jiang 2009). The GAF is a 90-point rating scale that assesses psychological, social, and occupational functioning. The possible score ranges from 0 to 100 , with higher scores indicating better functioning.

\section{Service use}

One study reported the number of participants who were readmitted (Goswami 2003).

\section{Adverse events}

Only one study reported the number of general adverse events (Zhang 2012), and five studies reported the average endpoint score for general adverse events assessed by the Treatment Emergent Symptom Scale (TESS) (Cai 2008; Jiang 2009; Jiang 2013; Zhang 
2010; Zhang 2012). The TESS is a valid instrument to assess adverse events, with higher scores indicating more severe adverse events.

Seven studies reported the number of specific adverse events (Chen 2012; Jiang 2009; Lin 2014; Petrides 2015; Wang 2011; Wang 2013; Yang 2005), and two studies reported the average endpoint score for specific adverse events assessed by TESS (Cai 2008; Zhang 2010).

\section{Excluded studies}

We excluded 49 studies (65 references) from the review for the following reasons (see Characteristics of excluded studies).

1. Seven studies were not randomised controlled trials (Arato 1980; Bhatia 1987; ChiCTR-OPC-14005339; Heath 1964; Smith 1967; Swoboda 2001; Zhou 2003).

2. In 10 studies (with 18 references), the participants were not all diagnosed with schizophrenia or a related disorder (Brill 1959; d'Elia 1970; Gander 1967; Girish 2003; Hargreaves 1972; Krystal 1993; Laurell 1970; Laurell 1970a; Reichert 1976; Ulett 1956).

3. In 26 studies (with 31 references), the participants were not diagnosed with treatment-resistant schizophrenia (Abraham 1987; Abrams 1967; Agarwal 1985; Bagadia 1988; Baker 1958; Baker 1960; Baker 1960a; Brandon 1985; Chatterjee 1980; Doongaji 1973; El Islam 1970; Gan 2017; Gangadhar 2000; Janakiramaiah 1981; Janakiramaiah 1982; Marjerrison 1975; Miller 1953; Naidoo 1956; Natani 1983; Rami 2008; Sarkar 1994; Small 1968; Taylor 1980; Ukpong 2002; Wessels 1971; Xue 1985).

4. The comparisons in four studies (with six references) did not meet the inclusion criteria. In one of these studies the participants were receiving concurrent pharmacological interventions and other interventions, and ECT was not being exclusively randomised (Bagadia 1981). The three studies compared different forms of ECT that were not in the protocol (He 2001; NCT02159001; Stenback 1957).

5. Two studies (with three references) were in languages other than English or Chinese (Ungvari 1981; Zadeh 2006).

\section{Studies awaiting classification}

See Characteristics of studies awaiting classification.

There were six studies awaiting classification due to unclear diagnosis, study design, or comparison (Chen 2006; Melzer 2015; Nicholas 1996; Oleneva 2005; Peyman 1956; Sackeim 2000). The contact information for these studies was not available.

\section{Ongoing studies}

See Characteristics of ongoing studies.

We identified three ongoing studies that began in 2000, 2008, and 2016 respectively, but had not yet been published. The location of these studies was the USA (NCT00042224), Thailand (NCT00753051), and China (NCT02926976). The contact information for these studies was not available, and NCT02926976 had not yet started recruiting as of the writing of this review.

\section{Risk of bias in included studies}

Details of the 'Risk of bias' assessments can be found in the 'Risk of bias' table corresponding to each study in the Characteristics of included studies tables, and in the 'Risk of bias' graph (Figure 2) and summary (Figure 3).

Figure 2. Risk of bias graph: review authors' judgements about each risk of bias item presented as percentages across all included studies.

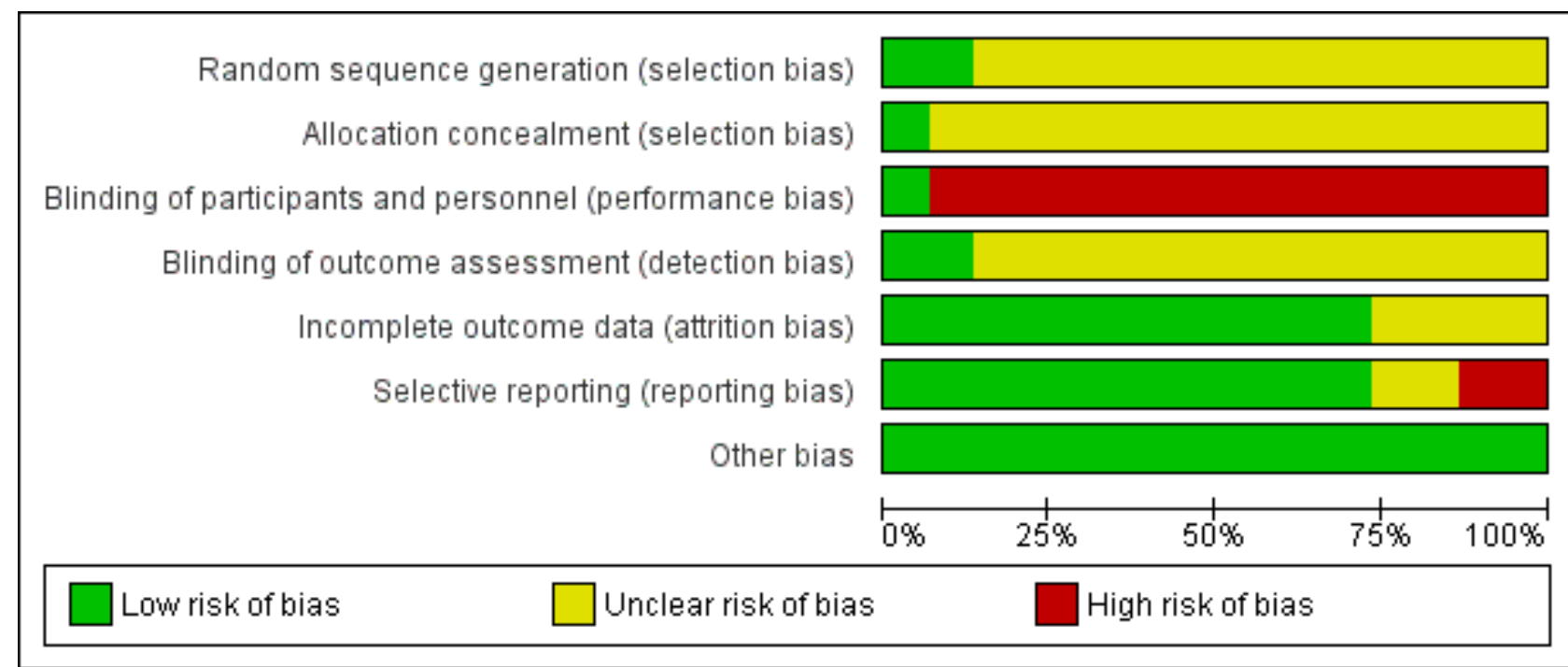


Figure 3. Risk of bias summary: review authors' judgements about each risk of bias item for each included study.

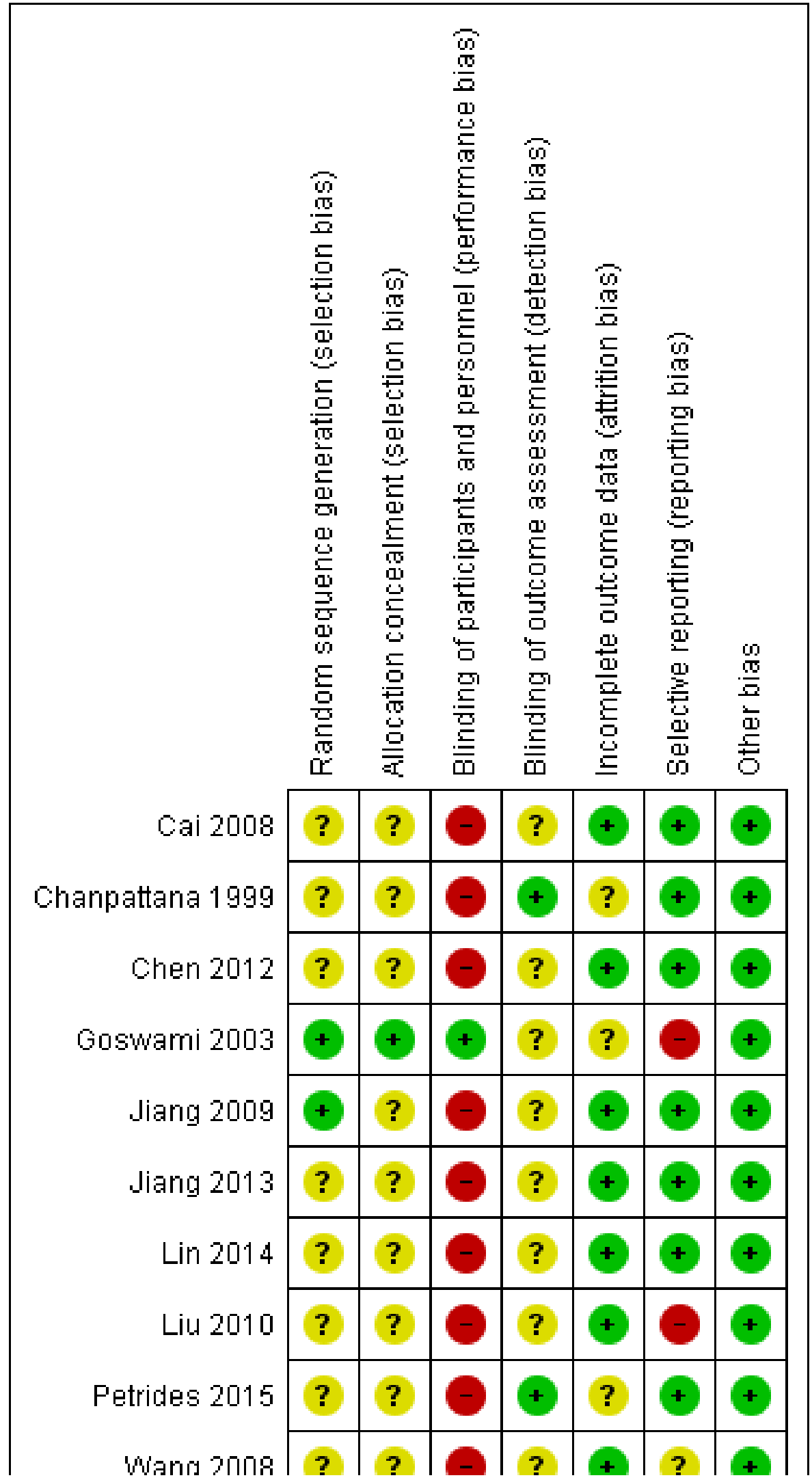


Figure 3. (Continued)

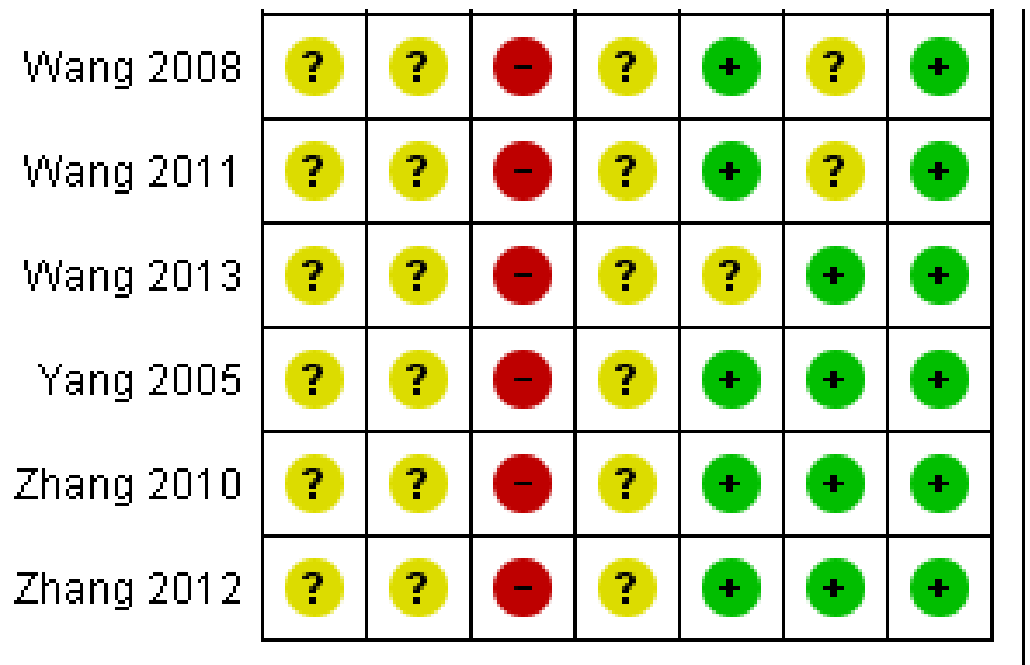

\section{Allocation}

\section{Random sequence generation}

All 15 included studies reported some form of randomisation. Two studies reported adequate sequence generation, which we rated as at low risk of bias. The methods used to generate the allocation sequence were random number tables (Goswami 2003; Jiang 2009). The remaining 13 studies provided insufficient information to assess bias in this domain, and we classified them as at unclear risk of bias.

\section{Allocation concealment}

Only Goswami 2003 reported adequate allocation concealment, where allocation was managed by a person who was not involved in carrying out the study. We rated this study as at low risk of bias. The remaining 14 studies provided insufficient information to assess risk of bias for this domain and were classified as at unclear risk of bias.

\section{Blinding}

\section{Blinding of participants and personnel}

Only Goswami 2003 reported that both participants and personnel were double-blinded, and that a sham-ECT was used as a control to ensure the blinding. We rated this study as at low risk of bias. In the remaining 14 studies, participants in one group received ECT, and the other group did not. It would not have been possible to blind participants and healthcare professionals administering the treatment. We rated these studies as at high risk of performance bias.

\section{Blinding of outcome assessment}

Two studies reported that the outcome assessors were blinded to the treatment, which we rated as at low risk of bias (Chanpattana 1999; Petrides 2015). The remaining 13 studies provided insufficient information to assess bias in this domain and were classified as at unclear risk of detection bias.

\section{Incomplete outcome data}

We rated 11 studies as at low risk of bias for this domain: nine studies had no missing outcome data (Cai 2008; Chen 2012; Jiang 2013; Lin 2014; Liu 2010; Wang 2008; Wang 2011; Yang 2005; Zhang 2012), while in the other two studies the proportion of participants who dropped was less than $10 \%$, or the reasons for dropping were not relevant to the effect of the intervention (Jiang 2009; Zhang 2010).

We rated three studies (in which the attrition rate was more than $10 \%$ but less than 20\%) as at unclear risk of attrition bias. Chanpattana 1999 performed an ITT analysis, but the number of participants randomised to each group was not reported. Goswami 2003 did not provide a reason for the attrition and did not perform an ITT analysis. In Petrides 2015, the reasons for attrition varied between groups.

We also rated the remaining study, Wang 2013, as at unclear risk of attrition bias. Although the attrition rate was not described, there was one additional participant in one treatment group, and two fewer participants in another treatment group according to the reported results.

\section{Selective reporting}

Most of the study protocols were not available. We rated 11 studies that had appropriately reported all the outcomes stated in the methods section of the study to be at low risk of bias for this domain (Cai 2008; Chanpattana 1999; Chen 2012; Jiang 2009; Jiang 2013; Lin 2014; Petrides 2015; Wang 2013; Yang 2005; Zhang 2010; Zhang 2012).

We rated two studies as at unclear risk of bias because they did not report memory assessment in the control groups (Wang 2008; Wang 2011). The primary outcome (response to treatment) was not reported in the two remaining studies (Goswami 2003; Liu 2010), therefore these studies were rated as at high risk of reporting bias.

\section{Other potential sources of bias}

We did not find any other obvious bias in the included studies and therefore rated this domain as at low risk of bias. 


\section{Effects of interventions}

See: Summary of findings for the main comparison ECT plus standard care versus sham-ECT plus standard care for treatmentresistant schizophrenia; Summary of findings 2 ECT plus standard care versus antipsychotics plus standard care for treatmentresistant schizophrenia; Summary of findings 3 ECT plus standard care versus standard care for treatment-resistant schizophrenia; Summary of findings 4 ECT alone versus antipsychotics for treatment-resistant schizophrenia

\section{COMPARISON 1: ECT plus standard care versus sham-ECT plus standard care}

For this comparison, we found only one relevant study involving 25 participants who received either ECT combined with chlorpromazine or sham-ECT combined with chlorpromazine (Goswami 2003). This was a short course of ECT performed as continuation with bilateral placement of electrodes. The participants in this study were strictly diagnosed with schizophrenia by DSM-IV.

See Summary of findings for the main comparison.

\subsection{Mental state - total score (BPRS, high = poor) - short term}

Goswami 2003 reported the endpoint score of participants' mental state assessed by the BPRS at short-term follow-up. We found no clear difference in the BPRS score for mental state between the ECT and the sham-ECT groups (mean difference (MD) 3.60, 95\% confidence interval $(\mathrm{Cl})-3.69$ to 10.89 ; participants $=25$; studies $=1$; very low-quality evidence; Analysis 1.1).

\subsection{Service use - number readmitted - short term}

Goswami 2003 reported the number of participants who were readmitted at short-term follow-up. There were fewer readmitted participants in the ECT group than in the sham-ECT group (risk ratio (RR) $0.29,95 \% \mathrm{Cl} 0.10$ to 0.85 ; participants $=25$; studies $=1$; lowquality evidence; Analysis 1.2).

For this comparison, Goswami 2003 did not report other predefined outcomes, including response to treatment, cognitive functioning, satisfaction and acceptability of treatment, general functioning, and adverse events.

\section{COMPARISON 2: ECT plus standard care versus antipsychotic (clozapine) plus standard care}

For this comparison, we found only one relevant study involving 162 participants who received either modified ECT (MECT) combined with ziprasidone or clozapine combined with ziprasidone (Jiang 2013). The short course of ECT was performed as continuation. The electrode placement was not described in this study. The participants in this study were strictly diagnosed with schizophrenia by CCMD-3.

See Summary of findings 2 .

\subsection{Response to treatment - clinically significant response (BPRS} reducing rate $\geq 50 \%$ ) - medium term

Jiang 2013 reported that there was no clear medium-term difference in the number of clinically significant responders between the MECT and clozapine treatment groups (RR 1.23, 95\%
$\mathrm{Cl} 0.95$ to 1.58 ; participants $=162 ;$ studies $=1$; low-quality evidence; Analysis 2.1).

\subsection{Mental state - total score (BPRS, high = poor) - short term}

Jiang 2013 reported the endpoint score of participants' mental state assessed by the BPRS. The study found a lower short-term total BPRS score for mental state after MECT treatment than after clozapine treatment $(\mathrm{MD}-5.20,95 \% \mathrm{Cl}-7.93$ to -2.47 ; participants $=$ 162 ; studies $=1$; very low-quality evidence; Analysis 2.2).

\subsection{Mental state - total score (BPRS, high = poor) - medium term (skewed data)}

Jiang 2013 also reported a total medium-term BPRS score for mental state. However, as the data were skewed, a parameter test was not applicable. We have presented data in Analysis 2.3.

\subsection{Mental state - specific symptom score (BPRS, high = poor) - short term}

Jiang 2013 found lower short-term BPRS scores for specific mental symptoms in the MECT group than in the clozapine group (Analysis 2.4).

\subsubsection{Anxiety and depression}

Jiang 2013 found lower short-term BPRS scores on anxiety and depression in the MECT group than in the clozapine group (MD $-0.60,95 \% \mathrm{Cl}-1.12$ to -0.08 ; participants $=162$; studies $=1$ ).

\subsubsection{Lack of vitality}

Jiang 2013 found lower short-term BPRS scores on lack of vitality in the MECT group than in the clozapine group (MD -2.20, 95\% Cl-2.74 to -1.66 ; participants $=162$; studies $=1$ ).

\subsubsection{Thought disturbance}

Jiang 2013 found lower short-term BPRS scores on thought disturbance in the MECT group than in the clozapine group (MD $-0.70,95 \% \mathrm{Cl}-1.32$ to -0.08 ; participants $=162$; studies $=1$ ).

\subsubsection{Agitation}

Jiang 2013 found lower short-term BPRS scores on agitation in the MECT group than in the clozapine group (MD $-0.70,95 \% \mathrm{Cl}-0.82$ to -0.58 ; participants $=162$; studies $=1$ )

\subsubsection{Hostile suspiciousness}

Jiang 2013 found lower short-term BPRS scores on hostile suspiciousness in the MECT group than in the clozapine group (MD $-1.00,95 \% \mathrm{Cl}-1.56$ to -0.44 ; participants $=162$; studies $=1$ ).

\subsection{Mental state - specific symptom score (BPRS, high = poor) - medium term}

Jiang 2013 found lower medium-term BPRS scores for specific mental symptoms in the MECT group than in the clozapine group (Analysis 2.5).

\subsubsection{Anxiety and depression}

Jiang 2013 found lower medium-term BPRS scores on anxiety and depression in the MECT group than in the clozapine group (MD $-1.50,95 \% \mathrm{Cl}-1.73$ to -1.27 ; participants $=162$; studies $=1$ ). 


\subsubsection{Lack of vitality}

Jiang 2013 found lower medium-term BPRS scores on lack of vitality in the MECT group than in the clozapine group (MD -1.20, $95 \% \mathrm{Cl}-1.47$ to -0.93 ; participants $=162$; studies $=1$ ).

\subsubsection{Thought disturbance}

Jiang 2013 found lower medium-term BPRS scores on thought disturbance in the MECT group than in the clozapine group (MD $-1.70,95 \% \mathrm{Cl}-2.12$ to -1.28 ; participants $=162$; studies $=1$ ).

\subsubsection{Agitation}

Jiang 2013 found lower medium-term BPRS scores on agitation in the MECT group than in the clozapine group (MD -1.60, 95\% Cl-1.79 to -1.41 ; participants $=162$; studies $=1$ ).

\subsubsection{Hostile suspiciousness}

Jiang 2013 found lower medium-term BPRS scores on hostile suspiciousness in the MECT group than in the clozapine group (MD $-2.80,95 \% \mathrm{Cl}-3.14$ to -2.46 ; participants $=162$; studies $=1$ ).

\subsection{Adverse events - total score (TESS, high = poor)}

Jiang 2013 reported the endpoint score of adverse events assessed by TESS (Analysis 2.6).

\subsubsection{Short term}

Jiang 2013 found no clear short-term difference on TESS scores between the two groups (MD $-0.40,95 \% \mathrm{Cl}-0.91$ to 0.11 ; participants $=162$; studies $=1$ ).

\subsubsection{Medium term}

Jiang 2013 found lower medium-term TESS scores in the MECT group than in the clozapine group (MD -1.10, $95 \% \mathrm{Cl}-1.40$ to -0.80 ; participants $=162$; studies $=1$ ).

For this comparison, Jiang 2013 did not report other predefined outcomes, including cognitive functioning, satisfaction and acceptability of treatment, general functioning, and service outcomes.

\section{COMPARISON 3: ECT plus standard care versus standard care}

For this comparison, we found 12 relevant studies that compared ECT plus standard care with standard care, involving 1020 participants.

\section{See Summary of findings 3.}

\subsection{Response to treatment - clinically significant response (as defined by each of the studies)}

Ten studies reported useable data on this outcome (Analysis 3.1).

\subsubsection{Short term}

Wang 2013 reported the short-term clinically significant responders rate. The study found that there were more responders in the ECT group than in the group that did not receive ECT (RR 1.91, 95\% Cl 1.09 to 3.36 ; participants $=72$; studies $=1$ ).

\subsubsection{Medium term}

Nine studies reported the medium-term clinically significant responders rate (Cai 2008; Chen 2012; Jiang 2009; Petrides 2015;
Wang 2008; Wang 2011; Yang 2005; Zhang 2010; Zhang 2012). There were also more responders in the ECT group than in the group that did not receive ECT (RR 2.06, $95 \% \mathrm{Cl} 1.75$ to 2.42; participants = 819; studies $=9$; moderate-quality evidence).

\subsection{Cognitive functioning - memory deterioration - short term}

Wang 2013 reported that there were more participants with memory deterioration in the ECT group than in the group that did not receive ECT at short-term follow-up (RR $27.00,95 \% \mathrm{Cl} 1.67$ to 437.68; participants $=72$; studies $=1$; very low-quality evidence; Analysis 3.2).

\subsection{Cognitive functioning - specific symptom score (WCST, high = poor, other than correct number) - medium term}

Jiang 2009 reported the medium-term endpoint scores from sub scales of the WCST (Analysis 3.3).

\subsubsection{Total test number}

Jiang 2009 found that participants who received ECT had lower scores in total test number at medium-term follow-up (MD -8.74, $95 \% \mathrm{Cl}-16.88$ to -0.60 ; participants $=67$; studies $=1$ ).

\subsubsection{Correct number}

Jiang 2009 found that there were no clear differences in the correct number between the two groups at medium-term follow-up (MD $3.03,95 \% \mathrm{Cl}-0.15$ to 6.21 ; participants $=67$; studies $=1$ ).

\subsubsection{Categories completed}

Jiang 2009 found that participants who received ECT had lower scores in categories completed at medium-term follow-up (MD $-0.36,95 \% \mathrm{Cl}-0.49$ to -0.23 ; participants $=67$; studies $=1$ ).

\subsection{Cognitive functioning - specific symptom score (WCST, high = poor) - medium term (skewed data)}

Data reported by Jiang 2009 ( $n=67$ ) on perseveration and nonperseveration errors were skewed; we have only presented them as 'other data' without the parameter test (Analysis 3.4).

\subsection{Cognitive functioning - memory (WMS, high = good) - short term}

Zhang 2010 reported the short-term endpoint memory scores from sub scales of the WMS. There were no clear differences in specific memory symptoms between the two groups (Analysis 3.5).

\subsubsection{Memory quotient (MQ)}

Zhang 2010 found no clear differences in MQ between the two groups (MD 0.27, 95\% Cl -3.54 to 4.08; participants $=245$; studies $=$ $1)$.

\subsubsection{Memorise number (1 to 100 )}

Zhang 2010 found no clear differences in memorise number (1 to 100 ) between the two groups (MD $-0.09,95 \% \mathrm{Cl}-0.71$ to 0.53 ; participants $=245$; studies $=1$ ).

\subsubsection{Memorise number ( 100 to 1 )}

Zhang 2010 found no clear differences in memorise number $(100$ to 1 ) between the two groups (MD $-0.30,95 \% \mathrm{Cl}-0.98$ to 0.38 ; participants $=245$; studies $=1$ ). 


\subsubsection{Accumulation of memory}

Zhang 2010 found no clear differences in accumulation of memory between the two groups (MD $-0.11,95 \% \mathrm{Cl}-0.73$ to 0.51 ; participants $=245 ;$ studies $=1$ ).

\subsubsection{Picture memory}

Zhang 2010 found no clear differences in picture memory between the two groups (MD $-0.41,95 \% \mathrm{Cl}-1.01$ to 0.19 ; participants $=245$; studies $=1$ ).

\subsubsection{Recognition memory}

Zhang 2010 found no clear differences in recognition memory between the two groups (MD $-0.03,95 \% \mathrm{Cl}-0.64$ to 0.58 ; participants $=245$; studies $=1$ ).

\subsubsection{Regeneration memory}

Zhang 2010 found no clear differences in regeneration memory between the two groups (MD $-0.04,95 \% \mathrm{Cl}-0.78$ to 0.70 ; participants $=245$; studies $=1$ ).

\subsubsection{Associative memory}

Zhang 2010 found no clear differences in associative memory between the two groups (MD $-0.09,95 \% \mathrm{Cl}-0.97$ to 0.79 ; participants $=245$; studies $=1$ ).

\subsubsection{Haptic memory}

Zhang 2010 found no clear differences in haptic memory between the two groups (MD $-0.07,95 \% \mathrm{Cl}-0.53$ to 0.39 ; participants $=245$; studies $=1$ )

\subsubsection{Understanding memory}

Zhang 2010 found no clear differences in understanding memory between the two groups (MD $0.02,95 \% \mathrm{Cl}-0.56$ to 0.60 ; participants $=245$; studies $=1$ ).

\subsubsection{Memorise random number}

Zhang 2010 found no clear differences in memorise random number between the two groups (MD $0.33,95 \% \mathrm{Cl}-0.29$ to 0.95 ; participants $=245$; studies $=1$ ).

\subsection{Cognitive functioning - memory (WMS, high = good) - medium term}

Zhang 2010 reported the medium-term endpoint memory scores from sub scales of the WMS. There were no clear differences in specific memory symptoms between the two groups (Analysis 3.6).

\subsubsection{Memory quotient (MQ)}

Zhang 2010 found no clear differences in MQ between the two groups (MD 1.75, 95\% Cl -2.24 to 5.74; participants $=245$; studies $=$ $1)$.

\subsubsection{Memorise number ( 1 to 100 )}

Zhang 2010 found no clear differences in memorise number (1 to 100 ) between the two groups (MD $-0.06,95 \% \mathrm{Cl}-0.69$ to 0.57 ; participants $=245$; studies $=1$ )

\subsubsection{Memorise number (100 to 1 )}

Zhang 2010 found no clear differences in memorise number (100 to 1 ) between the two groups (MD $-0.15,95 \% \mathrm{Cl}-0.88$ to 0.58 ; participants $=245$; studies $=1$ ).

\subsubsection{Accumulation of memory}

Zhang 2010 found no clear differences in accumulation of memory between the two groups (MD 0.03, $95 \% \mathrm{Cl}-0.59$ to 0.65 ; participants $=245$; studies $=1$ ).

\subsubsection{Picture memory}

Zhang 2010 found no clear differences in picture memory between the two groups (MD $-0.41,95 \% \mathrm{Cl}-1.01$ to 0.19 ; participants $=245$; studies $=1$ ).

\subsubsection{Recognition memory}

Zhang 2010 found no clear differences in recognition memory between the two groups (MD 0.09, $95 \% \mathrm{Cl}-0.52$ to 0.70 ; participants $=245 ;$ studies $=1$ ).

\subsubsection{Regeneration memory}

Zhang 2010 found no clear differences in regeneration memory between the two groups (MD 0.11, $95 \% \mathrm{Cl}-0.64$ to 0.86 ; participants $=245$; studies $=1$ ).

\subsubsection{Associative memory}

Zhang 2010 found no clear differences in associative memory between the two groups (MD 0.11, $95 \% \mathrm{Cl}-0.79$ to 1.01; participants $=245$; studies $=1$ ).

\subsubsection{Haptic memory}

Zhang 2010 found no clear differences in haptic memory between the two groups (MD $0.10,95 \% \mathrm{Cl}-0.38$ to 0.58 ; participants $=245$; studies $=1$ )

\subsubsection{Understanding memory}

Zhang 2010 found no clear differences in understanding memory between the two groups (MD $-0.06,95 \% \mathrm{Cl}-0.63$ to 0.51 ; participants $=245 ;$ studies $=1$ ).

\subsubsection{Memorise random number}

Zhang 2010 found no clear differences in memorise random number between the two groups (MD $0.24,95 \% \mathrm{Cl}-0.37$ to 0.85 ; participants $=245$; studies $=1$ ).

\subsection{Satisfaction and acceptability of treatment - leaving the study early - medium term}

Three studies reported the number of participants who left the study early at medium-term follow-up (Jiang 2009; Petrides 2015; Zhang 2010). There was no clear difference between the two groups (RR 1.18, 95\% Cl 0.38 to 3.63; participants = 354; studies = 3; very low-quality evidence; Analysis 3.7).

\subsection{Mental state - total score (BPRS, high $=$ poor)}

Two studies reported mental state by measuring endpoint total scores of BPRS (Analysis 3.8). 


\subsubsection{Short term}

Cai 2008 and Zhang 2010 reported this outcome at short-term follow-up. We found that participants who received ECT had lower short-term BPRS scores than those who did not receive ECT (MD $-5.50,95 \% \mathrm{Cl}-6.99$ to -4.00 ; participants $=345$; studies $=2 ; 12=66 \%$ ). The result had important heterogeneity, and no obvious source was identified.

\subsubsection{Medium term}

Cai 2008 and Zhang 2010 reported this outcome at medium-term follow-up. We found that the participants who received ECT had lower medium-term BPRS scores than those who did not receive ECT (MD -11.18, 95\% Cl -12.61 to -9.76; participants = 345; studies = 2; low-quality evidence).

\subsection{Mental state - total score (BPRS, high = poor) - medium term (skewed data)}

We could not pool the BPRS scores in Chanpattana $1999(n=30)$ in the meta-analysis due to skewness; we have presented these in Analysis 3.9.

\subsection{Mental state - total score (MMSE, high = good) - medium term}

Two studies reported mental state by measuring endpoint total scores of MMSE at medium-term follow-up (Chanpattana 1999; Petrides 2015). We found that the participants who received ECT had higher MMSE scores than those who did not receive ECT (MD $0.98,95 \% \mathrm{Cl} 0.30$ to 1.65 ; participants $=69$; studies $=2$; Analysis 3.10).

\subsection{Mental state - total score (PANSS, high = poor)}

Six studies reported mental state by measuring endpoint total scores of PANSS (Analysis 3.11).

\subsubsection{Short term}

Six studies reported short-term total scores of PANSS (Chen 2012; Jiang 2009; Wang 2008; Wang 2011; Yang 2005; Zhang 2012). We found that the participants who received ECT had lower PANSS scores than those who did not receive ECT (MD $-11.41,95 \% \mathrm{Cl}$ -13.49 to -9.34 ; participants $=432$; studies $\left.=6 ; 1^{2}=94 \%\right)$. The result had important heterogeneity. The statistical heterogeneity was reduced to $1^{2}=0 \%$ after removing Yang 2005 from the meta-analysis (MD $-4.96,95 \% \mathrm{Cl}-7.48$ to -2.44 ; participants = 372; studies $=5$ ). Accordingly, the treatment effect was robust to heterogeneity amongst the studies. The participants in Yang 2005 were diagnosed with treatment-resistant schizophrenia with agitation and aggressive behaviour, which may be the source of the heterogeneity.

\subsubsection{Medium term}

Six studies reported medium-term total scores of PANSS (Chen 2012; Jiang 2009; Wang 2008; Wang 2011; Yang 2005; Zhang 2012). We found that the participants who received ECT had lower PANSS scores than those who did not receive ECT (MD -7.62, 95\% Cl -9.49 to -5.74 ; participants $=432$; studies $=6$ ).

\subsection{Mental state - specific symptom score (BPRS, high = poor) - short term}

Three studies reported short-term BPRS sub scale scores for specific mental symptoms. We found that the participants who received ECT had lower BPRS scores for specific symptoms compared with those who did not receive ECT (Analysis 3.12).

\subsubsection{Anxiety and depression}

Cai 2008 and Zhang 2010 reported data on anxiety and depression. We found that the participants who received ECT had lower BPRS scores for anxiety and depression compared with those who did not receive ECT (MD - $0.97,95 \% \mathrm{Cl}-1.28$ to -0.65 ; participants = 345; studies $\left.=2 ; 1^{2}=77 \%\right)$. The result had important heterogeneity, and no obvious source was identified.

\subsubsection{Lack of vitality}

Cai 2008 and Zhang 2010 reported data on lack of vitality. We found that the participants who received ECT had lower BPRS scores for lack of vitality compared with those who did not receive ECT (MD $-2.40,95 \% \mathrm{Cl}-2.71$ to -2.09 ; participants $=345$; studies $=2 ; \mathrm{I}^{2}=77 \%$ ). The result had important heterogeneity, and no obvious source was identified.

\subsubsection{Thought disturbance}

Cai 2008 and Zhang 2010 reported data on thought disturbance. We found that the participants who received ECT had lower BPRS scores for thought disturbance compared with those who did not receive ECT (MD $-0.71,95 \% \mathrm{Cl}-1.06$ to -0.35 ; participants $=345$; studies $=2$ ).

\subsubsection{Agitation}

Cai 2008 and Zhang 2010 reported data on agitation. We found that the participants who received ECT had lower BPRS scores for agitation compared with those who did not receive ECT (MD -1.23, $95 \% \mathrm{Cl}-1.51$ to -0.95 ; participants $=345$; studies $=2$ ).

\subsubsection{Hostile suspiciousness}

Cai 2008 and Zhang 2010 reported data on hostile suspiciousness. We found that the participants who received ECT had lower BPRS scores for hostile suspiciousness compared with those who did not receive ECT (MD $-0.57,95 \% \mathrm{Cl}-0.91$ to -0.24 ; participants = 345; studies $=2$ ).

\subsubsection{Psychosis}

Petrides 2015 reported data on psychosis. We found that the participants who received ECT had lower BPRS scores for psychosis compared with those who did not receive ECT (MD -5.24, 95\% Cl -5.89 to -4.59 ; participants $=39$; studies $=1$ ).

\subsection{Mental state - specific symptom score (BPRS, high = poor) - medium term}

Four studies reported medium-term BPRS sub scale scores for specific mental symptoms. We found that the participants who received ECT had lower BPRS scores for specific symptoms compared with those who did not receive ECT (Analysis 3.13).

\subsubsection{Anxiety and depression}

Three studies reported data on anxiety and depression (Cai 2008; Lin 2014; Zhang 2010). We found that the participants who received 
ECT had lower BPRS scores for anxiety and depression compared with those who did not receive ECT (MD $-1.50,95 \% \mathrm{Cl}-1.72$ to -1.29 ; participants $=423$; studies $\left.=3 ;\left.\right|^{2}=76 \%\right)$. The result had important heterogeneity, and no obvious source was identified. However, the treatment effect was robust to heterogeneity amongst the studies. The statistical heterogeneity was reduced to $\mathrm{L}^{2}=0 \%$ after removing Cai 2008 from the meta-analysis (MD $-1.61,95 \% \mathrm{Cl}-1.84$ to -1.39 ; participants $=323$; studies $=2$ ).

\subsubsection{Lack of vitality}

Three studies reported data on lack of vitality (Cai 2008; Lin 2014; Zhang 2010). We found that the participants who received ECT had lower BPRS scores for lack of vitality compared with those who did not receive ECT (MD -1.80, 95\% Cl -2.02 to -1.58; participants = 423; studies $=3 ; 12=87 \%)$. The result had important heterogeneity, and no obvious source was identified. However, the treatment effect was robust to heterogeneity amongst the studies. The statistical heterogeneity was reduced to $\mathrm{I}^{2}=0 \%$ after removing Lin 2014 from the meta-analysis (MD $-2.14,95 \% \mathrm{Cl}-2.42$ to -1.86 ; participants = 345 ; studies $=2$ ).

\subsubsection{Thought disturbance}

Three studies reported data on thought disturbance (Cai 2008; Lin 2014; Zhang 2010). We found that the participants who received ECT had lower BPRS scores for thought disturbance compared with those who did not receive ECT (MD $-2.69,95 \% \mathrm{Cl}-2.95$ to -2.43 ; participants $=423$; studies $\left.=3 ; 1^{2}=85 \%\right)$. The statistical heterogeneity was reduced to $\mathrm{I}^{2}=0 \%$ after removing Lin 2014 from the meta-analysis $(\mathrm{MD}-2.94,95 \% \mathrm{Cl}-3.23$ to -2.64 ; participants = 345 ; studies $=2$ ).

\subsubsection{Agitation}

Three studies reported data on agitation (Cai 2008; Lin 2014; Zhang 2010). We found that the participants who received ECT had lower BPRS scores for agitation compared with those who did not receive ECT (MD -1.73, 95\% Cl -1.91 to -1.54 ; participants $=423$; studies $=3$ ).

\subsubsection{Hostile suspiciousness}

Three studies reported data on hostile suspiciousness (Cai 2008; Lin 2014; Zhang 2010). We found that the participants who received ECT had lower BPRS scores for hostile suspiciousness compared with those who did not receive ECT (MD $-2.77,95 \% \mathrm{Cl}-3.00$ to -2.54 ; participants $=423$; studies $=3$ ).

\subsubsection{Psychosis}

Petrides 2015 reported data on psychosis. We found that the participants who received ECT had lower BPRS scores for psychosis compared with those who did not receive ECT (MD -6.07, 95\% Cl -6.72 to -5.42 ; participants $=39$; studies $=1$ ).

\subsection{Mental state - specific symptom score (PANSS, high = poor) - short term}

Six studies reported useable short-term PANSS sub scale scores for specific mental symptoms (Analysis 3.14).

\subsubsection{Positive symptom}

Three studies reported data on positive symptom (Chen 2012; Wang 2008; Zhang 2012). We found no clear differences in short-term PANSS scores for positive symptom between the two groups (MD $-1.56,95 \% \mathrm{Cl}-3.12$ to 0.00 ; participants $=231$; studies $=3$ ).

\subsubsection{Negative symptom}

Five studies reported data on negative symptom (Chen 2012; Jiang 2009; Wang 2008; Wang 2011; Zhang 2012). We found no clear differences in short-term PANSS scores for negative symptom between the two groups (MD $-1.03,95 \% \mathrm{Cl}-2.42$ to 0.35 ; participants $=372$; studies $=5$ ).

\subsubsection{General psychopathology}

Three studies reported data on general psychopathology (Wang 2008; Wang 2011; Zhang 2012). We found no clear differences in short-term PANSS scores for general psychopathology between the two groups (MD $-1.59,95 \% \mathrm{Cl}-3.83$ to 0.66 ; participants $=234$; studies $=3)$.

\subsubsection{Excitement}

Yang 2005 reported that participants with agitation and aggressive behaviour who received ECT had lower short-term PANSS scores for excitement than those who did not receive ECT (MD $-5.79,95 \% \mathrm{Cl}$ -7.34 to -4.24 ; participants $=60$; studies $=1$ ).

\subsubsection{Hostility}

Yang 2005 reported that participants with agitation and aggressive behaviour who received ECT had lower short-term PANSS scores for hostility than those who did not receive ECT (MD -2.48, 95\% Cl -3.93 to -1.03 ; participants $=60$; studies $=1$ ).

\subsection{Mental state - specific symptom score (PANSS, high = poor) - short term (skewed data)}

Data reported by other studies for positive symptom and general psychopathology at short-term follow-up were skewed and are presented as 'other data' (Analysis 3.15) (Chen 2012; Jiang 2009; Wang 2011).

\subsection{Mental state - specific symptom score (PANSS, high = poor) - medium term}

Five studies reported useable medium-term PANSS sub scale scores for specific mental symptoms (Analysis 3.16).

\subsubsection{Negative symptom}

Three studies reported data on negative symptom (Chen 2012; Wang 2011; Zhang 2012). We found no clear differences in mediumterm PANSS scores for negative symptom between the two groups (MD 0.58, $95 \% \mathrm{Cl}-1.47$ to 2.62; participants $=229$; studies $=3$ ).

\subsubsection{General psychopathology}

Two studies reported data on general psychopathology (Wang 2008; Wang 2011). We found that participants who received ECT had lower medium-term PANSS scores for general psychopathology than those who did not receive ECT (MD - $2.93,95 \% \mathrm{Cl}-5.32$ to -0.54 ; participants $=150$; studies $=2$ ).

\subsubsection{Excitement}

Yang 2005 reported that participants with agitation and aggressive behaviour who received ECT had lower medium-term PANSS scores for excitement than those who did not receive ECT (MD -2.31, 95\% $\mathrm{Cl}-3.44$ to -1.18 ; participants $=60$; studies $=1$ ). 


\subsubsection{Hostility}

Yang 2005 reported that participants with agitation and aggressive behaviour who received ECT had lower medium-term PANSS scores for hostility than those who did not receive ECT (MD $-2.81,95 \% \mathrm{Cl}$ -4.05 to -1.57 ; participants $=60$; studies $=1$ ).

3.17 Mental state - specific symptom score (PANSS, high = poor) medium term (skewed data)

Five studies also reported the medium-term PANSS scores for positive symptoms (Chen 2012; Jiang 2009; Wang 2008; Wang 2011; Zhang 2012); however, as the data were skewed, we have presented them as 'other data' in Analysis 3.17.

\subsection{Mental state - specific symptom score (SAPS, high = poor) short term}

Only one study reported the SAPS scores for positive symptoms at short-term follow-up (Analysis 3.18) (Liu 2010).

\subsubsection{Hallucinations}

Liu 2010 reported that participants who received ECT had lower short-term SAPS scores for hallucinations than those who did not receive $\mathrm{ECT}$ (MD $-1.50,95 \% \mathrm{Cl}-1.71$ to -1.29 ; participants $=72$; studies $=1)$.

\subsubsection{Delusions}

Liu 2010 reported that participants who received ECT had lower short-term SAPS scores for delusions than those who did not receive ECT (MD $-2.81,95 \% \mathrm{Cl}-3.10$ to -2.52 ; participants $=72$; studies $=1)$.

\subsubsection{Bizarre behaviour}

Liu 2010 reported that there was no clear difference in short-term SAPS scores for bizarre behaviour between the two groups (MD $-0.10,95 \% \mathrm{Cl}-0.21$ to 0.01 ; participants $=72$; studies $=1$ ).

\subsubsection{Positive formal thought disorder}

Liu 2010 reported that participants who received ECT had lower short-term SAPS scores for positive formal thought disorder than those who did not receive ECT (MD $-2.20,95 \% \mathrm{Cl}-2.50$ to -1.90 ; participants $=72$; studies $=1$ ).

\subsection{Mental state - specific symptom score (SANS, high = poor) - short term}

Only one study reported the SANS scores for negative symptoms at short-term follow-up (Analysis 3.19) (Liu 2010).

\subsubsection{Affective flattening or blunting}

Liu 2010 reported that participants who received ECT had lower short-term SANS scores for affective flattening or blunting than those who did not receive ECT (MD $-4.70,95 \% \mathrm{Cl}-5.03$ to -4.37 ; participants $=72$; studies $=1$ ).

\subsubsection{Alogia}

Liu 2010 reported that there was no clear difference in short-term SANS scores for alogia between the two groups (MD $-0.20,95 \% \mathrm{Cl}$ -0.43 to 0.03 ; participants $=72$; studies $=1$ ).

\subsubsection{Avolition}

Liu 2010 reported that there was no clear difference in short-term SANS scores for avolition between the two groups (MD - $0.20,95 \%$ $\mathrm{Cl}-0.40$ to 0.00 ; participants $=72$; studies $=1$ ).

\subsubsection{Anhedonia}

Liu 2010 reported that participants who received ECT had lower short-term SANS scores for anhedonia than those who did not receive ECT (MD $-0.30,95 \% \mathrm{Cl}-0.44$ to -0.16 ; participants $=72$; studies $=1)$.

\subsubsection{Attention}

Liu 2010 reported that participants who received ECT had lower short-term SANS scores for attention than those who did not receive ECT (MD $-0.90,95 \% \mathrm{Cl}-0.97$ to -0.83 ; participants $=72$; studies $=1$ ).

\subsection{General functioning - total score (GAF, high = good)}

Two studies reported general functioning by measuring endpoint total scores of GAF (Analysis 3.20).

\subsubsection{Short term}

Jiang $2009(n=67)$ reported the endpoint total score of general functioning assessed by GAF. There was no clear difference in shortterm GAF scores between the two groups (MD 4.32, $95 \% \mathrm{Cl}-0.20$ to 8.84 ; participants $=67$; studies $=1$ ).

\subsubsection{Medium term}

Two studies reported the endpoint total score for this outcome assessed by GAF (Chanpattana 1999; Jiang 2009). We found that participants who received ECT had higher medium-term GAF scores than those who did not receive ECT (MD $10.66,95 \% \mathrm{Cl} 6.98$ to 14.34 ; participants $=97$; studies $=2 ; 1^{2}=80 \%$; very low-quality evidence) . The result had important heterogeneity. Chanpattana 1999 used a long course of ECT (MD 20.47, 95\% Cl 11.21 to 29.73; participants = 30; studies $=1$ ), while Jiang 2009 used a short course (MD 8.82, $95 \% \mathrm{Cl} 4.81$ to 12.83 ; participants $=67$; studies $=1$ ). Accordingly, the treatment effect was robust to heterogeneity between the studies.

\subsection{Adverse events - general - medium term}

Only one study reported the number of participants who had any adverse event at medium-term follow-up (Zhang 2012). This study found no clear difference in the incidence of adverse events between the two groups (RR $1.33,95 \% \mathrm{Cl} 0.86$ to 2.06; participants $=84$; studies $=1$; Analysis 3.21).

\subsection{Adverse events - total score (TESS, high = poor)}

Four studies reported useable data for this outcome (Analysis 3.22).

\subsubsection{Short term}

Three studies reported the short-term endpoint scores of adverse events assessed by TESS (Cai 2008; Jiang 2009; Zhang 2012). There was no clear difference in the TESS total score between the two groups (MD $-0.19,95 \% \mathrm{Cl}-0.96$ to 0.57 ; participants $=251$; studies $=3$ ).

\subsubsection{Medium term}

Four studies reported the medium-term endpoint scores of adverse events assessed by TESS (Cai 2008; Jiang 2009; Zhang 2010; Zhang 2012). We found that participants who received ECT had lower TESS 
total scores than those who did not receive ECT (MD $-0.63,95 \% \mathrm{Cl}$ -1.01 to -0.25 ; participants $=496$; studies $=4$ ).

\subsection{Adverse events - specific symptom - short term}

Only one study reported the number of participants who had adverse events at short-term follow-up (Wang 2013). There were no clear differences in the incidence of specific symptoms between the two groups (Analysis 3.23).

\subsubsection{Abnormal electrocardiogram}

There were no clear differences in the incidence of abnormal electrocardiogram between the two groups (RR $0.85,95 \% \mathrm{Cl} 0.44$ to 1.63 ; participants $=72$; studies $=1$.

\subsubsection{Abnormal liver function}

There were no clear differences in the incidence of abnormal liver function between the two groups (RR $0.67,95 \% \mathrm{Cl} 0.12$ to 3.75 ; participants $=72$; studies $=1$ ).

\subsubsection{Constipation}

There were no clear differences in the incidence of constipation between the two groups ( $\mathrm{RR} 0.75,95 \% \mathrm{Cl} 0.36$ to 1.56 ; participants $=72$; studies $=1$ ).

\subsubsection{Headache}

There were no clear differences in the incidence of headache between the two groups (RR 7.00, 95\% Cl 0.37 to 130.82; participants $=72$; studies $=1$ ).

\subsubsection{Lethargy}

There were no clear differences in the incidence of lethargy between the two groups (RR $0.80,95 \% \mathrm{Cl} 0.44$ to 1.46 ; participants $=72$; studies $=1$ )

\subsubsection{Nausea or vomiting}

There were no clear differences in the incidence of nausea or vomiting between the two groups (RR $2.00,95 \% \mathrm{Cl} 0.19$ to 21.09 ; participants $=72$; studies $=1$ ).

\subsubsection{Salivation}

There were no clear differences in the incidence of salivation between the two groups (RR $0.73,95 \% \mathrm{Cl} 0.33$ to 1.59; participants $=72$; studies $=1$ ).

\subsubsection{Weight gain}

There were no clear differences in the incidence of weight gain between the two groups (RR $0.40,95 \% \mathrm{Cl} 0.14$ to 1.16 ; participants $=72$; studies $=1$ ).

\subsection{Adverse events - specific symptom - medium term}

Six studies reported the number of participants who had specific adverse events at medium-term follow-up (Analysis 3.24).

\subsubsection{Abnormal electrocardiogram}

Four studies reported this outcome (Cai 2008; Jiang 2009; Wang 2011; Yang 2005). There was no clear difference between the two groups (RR 0.92, 95\% Cl 0.67 to 1.26; participants $=274$; studies $=4$ ).

\subsubsection{Abnormal electroencephalogram}

Yang 2005 reported this outcome. There was no clear difference between the two groups (RR $1.04,95 \% \mathrm{Cl} 0.82$ to 1.32 ; participants $=60$; studies $=1$ ).

\subsubsection{Abnormal liver function}

Three studies reported this outcome (Chen 2012; Wang 2011; Yang 2005). There was no clear difference between the two groups (RR $0.72,95 \% \mathrm{Cl} 0.30$ to 1.71 ; participants $=205$; studies $=3$ ).

\subsubsection{Blurred vision}

Jiang 2009 reported this outcome. There was no clear difference between the two groups (RR 1.03, 95\% $\mathrm{Cl} 0.28$ to 3.79; participants $=69$; studies $=1$ ).

\subsubsection{Breathing discomfort}

Lin 2014 reported that fewer participants who received ECT experienced breathing discomfort compared with those who did not receive ECT (RR $0.08,95 \% \mathrm{Cl} 0.01$ to 0.61 ; participants $=78$; studies $=1$ ).

\subsubsection{Cathisophobia}

Jiang 2009 reported this outcome. There was no clear difference between the two groups ( $R R 0.80,95 \% \mathrm{Cl} 0.34$ to 1.91 ; participants =69; studies $=1$ ).

\subsubsection{Constipation}

Two studies reported that fewer participants who received ECT experienced constipation compared with those who did not receive ECT (RR 0.76, 95\% Cl 0.61 to 0.95; participants = 145; studies = 2; $\left.\right|^{2}=83 \%$ ) (Chen 2012; Wang 2011). The results had important heterogeneity, and no obvious source was identified. Chen 2012 reported that fewer participants in the ECT group had constipation (RR 0.56, 95\% Cl 0.37 to 0.83; participants $=71$; studies $=1$ ), while Wang 2011 reported that there was no clear difference between the two groups (RR $0.97,95 \% \mathrm{Cl} 0.75$ to 1.24 ; participants $=74$; studies $=1$ ).

\subsubsection{Decreased activity}

Jiang 2009 reported this outcome. There was no clear difference between the two groups (RR $0.56,95 \% \mathrm{Cl} 0.23$ to 1.35 ; participants =69; studies $=1$ ).

\subsubsection{Dizziness}

Jiang 2009 reported this outcome. There was no clear difference between the two groups (RR $1.72,95 \% \mathrm{Cl} 0.70$ to 4.20 ; participants $=69$; studies $=1$ ).

\subsubsection{Dry mouth}

Jiang 2009 reported this outcome. There was no clear difference between the two groups (RR $1.03,95 \% \mathrm{Cl} 0.33$ to 3.24; participants =69; studies $=1$. .

\subsubsection{Headache}

Two studies reported that more participants who received ECT experienced headache compared with those who did not receive ECT (RR 5.47, 95\% Cl 1.47 to 20.39; participants = 147; studies = 2; $\left.\left.\right|^{2}=55 \%\right)$ (Jiang 2009; Lin 2014). The result had important heterogeneity, and no obvious source was identified. Jiang 2009 
reported that more participants in the ECT group had headaches (RR 19.54, 95\% Cl 1.18 to 323.15; participants = 69; studies = 1), while Lin 2014 reported that there was no clear difference between the two groups (RR 2.00, $95 \% \mathrm{Cl} 0.39$ to 10.29 ; participants $=78$; studies $=1)$.

\subsubsection{Indigestion}

Lin 2014 reported this outcome. There was no clear difference between the two groups (RR $0.50,95 \% \mathrm{Cl} 0.16$ to 1.53; participants $=78$; studies $=1$ ).

\subsubsection{Insomnia}

Jiang 2009 reported this outcome. There was no clear difference between the two groups ( $R R 0.05,95 \% \mathrm{Cl} 0.00$ to 0.80 ; participants $=69 ;$ studies $=1$ ).

\subsubsection{Lethargy}

Three studies reported this outcome (Chen 2012; Jiang 2009; Wang 2011). There was no clear difference between the two groups (RR $0.86,95 \% \mathrm{Cl} 0.70$ to 1.05 ; participants $=214$; studies $=3 ; 1^{2}=73 \%$ ). The result had important heterogeneity, and no obvious source was identified.

\subsubsection{Leucopenia}

Two studies reported this outcome (Chen 2012; Wang 2011). There was no clear difference between the two groups (RR $0.73,95 \% \mathrm{Cl}$ 0.18 to 3.03 ; participants $=145$; studies $=2$ ).

\subsubsection{Muscle rigidity}

Jiang 2009 reported this outcome. There was no clear difference between the two groups (RR $0.77,95 \% \mathrm{Cl} 0.37$ to 1.59 ; participants $=69$; studies $=1$ ).

\subsubsection{Nausea or vomiting}

Two studies reported this outcome (Jiang 2009; Lin 2014). There was no clear difference between the two groups (RR $0.79,95 \% \mathrm{Cl}$ 0.31 to 2.02; participants $=147$; studies $\left.=2 ;\left.\right|^{2}=60 \%\right)$. The result had important heterogeneity, and no obvious source was identified.

\subsubsection{Recurrence of pre-existing involuntary 'jerky' movements}

Petrides 2015 reported this outcome. There was no clear difference between the two groups (RR 2.86, 95\% $\mathrm{Cl} 0.12$ to 66.11 ; participants = 39; studies $=1$ ).

\subsubsection{Salivation}

Two studies reported this outcome (Chen 2012; Wang 2011). There was no clear difference between the two groups (RR $0.80,95 \% \mathrm{Cl}$ 0.57 to 1.14 ; participants $=145$; studies $=2$ ).

\subsubsection{Tremor}

Jiang 2009 reported this outcome. There was no clear difference between the two groups (RR $0.72,95 \% \mathrm{Cl} 0.31$ to 1.67 ; participants $=69$; studies $=1$ ).

\subsubsection{Weight gain}

Three studies reported this outcome (Chen 2012; Jiang 2009; Wang 2011). There was no clear difference between the two groups (RR $0.72,95 \% \mathrm{Cl} 0.46$ to 1.13 ; participants $=214$; studies $=3$ ).

\subsubsection{Others}

Two studies reported there was no clear difference in other adverse events between the two groups (RR $0.99,95 \% \mathrm{Cl} 0.44$ to 2.23; participants $=145$; studies $=2)($ Chen 2012; Wang 2011).

\subsection{Adverse events - specific symptom score (TESS, high = poor) - short term (skewed data)}

Cai 2008 ( $n=100$ ) reported the TESS scores for specific symptoms, including abnormal laboratory values, behavioural toxicity, symptoms of the cardiovascular system, symptoms of the nervous system, symptoms of a vegetative nervous system, and others. Because of skewed data, we did not perform parameter tests, and only presented this information as 'other data' (Analysis 3.25).

\subsection{Adverse events - specific symptom score (TESS, high = poor) - medium term}

Only one study reported this outcome at medium-term follow-up (Analysis 3.26) (Zhang 2010).

\subsubsection{Abnormal laboratory values}

Zhang 2010 reported this outcome. There was no clear difference between the two groups (MD $-0.01,95 \% \mathrm{Cl}-0.08$ to 0.06 ; participants $=245 ;$ studies $=1$ ).

\subsubsection{Behavioural toxicity}

Zhang 2010 reported that participants who received ECT had lower TESS scores for behavioural toxicity compared with those who did not receive ECT (MD $-0.31,95 \% \mathrm{Cl}-0.38$ to -0.24 ; participants $=245$; studies $=1$ ).

\subsubsection{Symptoms of cardiovascular system}

Zhang 2010 reported that participants who received ECT had lower TESS scores for symptoms of the cardiovascular system compared with those who did not receive ECT (MD $-0.50,95 \% \mathrm{Cl}-0.58$ to -0.42 ; participants $=245$; studies $=1$ ).

\subsubsection{Symptoms of nervous system}

Zhang 2010 reported this outcome. There was no clear difference between the two groups (MD $-0.02,95 \% \mathrm{Cl}-0.08$ to 0.04 ; participants $=245 ;$ studies $=1$ ).

\subsubsection{Symptoms of vegetative nervous system}

Zhang 2010 reported this outcome. There was no clear difference between the two groups (MD 0.03, $95 \% \mathrm{Cl}-0.05$ to 0.11 ; participants $=245$; studies $=1$ ).

\subsubsection{Others}

Zhang 2010 reported there was no clear difference in other adverse events between the two groups (MD $-0.02,95 \% \mathrm{Cl}-0.17$ to 0.13 ; participants $=245$; studies $=1$ ).

\subsection{Adverse events - specific symptom score (TESS, high = poor) - medium term (skewed data)}

As data reported in Cai $2008(n=100)$ were skewed, we did not perform parameter tests, and only presented this information in Analysis 3.27.

Service outcomes were not reported for this comparison. 
COMPARISON 4: ECT alone versus antipsychotic alone (flupenthixol)

For this comparison, we found only one relevant study involving 30 participants receiving either ECT alone or flupenthixol (Chanpattana 1999). This study evaluated the effects of a long course of ECT performed as continuation with bilateral electrode placement. The participants in this study were strictly diagnosed with schizophrenia by DSM-IV.

See Summary of findings 4.

\subsection{Mental state - total score (BPRS, high = poor) - medium term}

Chanpattana 1999 reported the medium-term endpoint total scores of mental state assessed by the BPRS. The study found no clear differences in BPRS scores between the two groups (MD -0.93, 95\% $\mathrm{Cl}-6.95$ to 5.09 ; participants $=30$; studies $=1$; very low-quality evidence; Analysis 4.1).

\subsection{Mental state - total score (MMSE, high = good) - medium term}

Chanpattana 1999 reported the medium-term endpoint total scores of mental state assessed by the BPRS. The study found no clear differences in MMSE scores between the two groups (MD -0.20, 95\% $\mathrm{Cl}-3.70$ to 3.30; participants $=30$; studies $=1$; Analysis 4.2).

\subsection{General functioning - total score $(G A F$, high $=$ good) - medium term}

Chanpattana 1999 reported the medium-term endpoint total score for general functioning assessed by GAF. The study found no clear differences in GAF scores between the two groups (MD - $0.66,95 \%$ $\mathrm{Cl}-3.60$ to 2.28 ; participants $=30$; studies $=1$; very low-quality evidence; Analysis 4.3).

For this comparison, Chanpattana 1999 did not report other predefined outcomes including response to treatment, cognitive functioning, satisfaction and acceptability of treatment, service outcomes, and adverse events.

\section{SUBGROUP: ECT plus standard care versus standard care (TEST FOR DIFFERENCE BETWEEN SHORT/LONG COURSE OF ECT)}

\subsection{Response to treatment - clinically significant response (as defined by each of the studies) - medium term}

We performed subgroup analysis on this primary outcome according to different treatment duration of ECT (Analysis 5.1).

\subsubsection{Short course of ECT}

Six studies used a short course of ECT (Cai 2008; Chen 2012; Jiang 2009; Wang 2008; Wang 2011; Yang 2005). There were more responders in the group that received a short course of ECT than in the group that did not receive ECT (RR $2.23,95 \% \mathrm{Cl} 1.78$ to 2.80 ; participants $=450$; studies $=6$ ).

\subsubsection{Long course of ECT}

Three studies used a long course of ECT (Petrides 2015; Zhang 2010; Zhang 2012). There were more responders in the group that received a long course of ECT than in the group that did not receive ECT (RR 1.87, 95\% Cl 1.49 to 2.36; participants = 369; studies =3).

We tested for subgroup differences between short and long course of ECT. There was no evidence of different effects of ECT between the two groups at medium-term follow-up $\left(\mathrm{Chi}^{2}=1.12, \mathrm{df}=1(\mathrm{P}=\right.$ $\left.0.29), 1^{2}=10.7 \%\right)$

The subgroup analyses on other predefined factors, comparisons, and outcomes were not applicable due to insufficient data.

\section{SENSITIVITY ANALYSIS: ECT plus standard care versus standard care}

\section{Implication of randomisation}

The sensitivity analysis was not applicable because all the included studies had a random component in the sequence generation process.

\section{Assumptions for lost binary data}

This sensitivity analysis was only applicable for one primary outcome (medium-term response to treatment). Results were consistent between the incorporation of lost data with and without an ITT analysis (Analysis 6.1).

\section{Risk of bias}

The sensitivity analysis was not applicable because all the included studies were rated as at high risk of bias in at least one domain.

\section{Imputed values}

The sensitivity analysis was not applicable because all the included studies were without imputed values.

\section{Fixed-effect versus random-effects model}

This sensitivity analysis was only applicable for one primary outcome (medium-term response to treatment). The results were consistent between fixed-effect and random-effects models (Analysis 7.1).

\section{Diagnostic criteria}

The sensitivity analysis was not applicable because all participants in the included studies had strictly diagnosed schizophrenia by international standards.

The sensitivity analyses on other comparisons were not applicable due to insufficient data.

\section{Assessment of reporting biases}

None of the comparisons included more than 10 studies reporting the same outcome, so we did not produce a funnel plot to assess reporting biases.

\section{DISCUSSION}

\section{Summary of main results}

\section{COMPARISON 1: ECT plus standard care versus sham-ECT plus} standard care

For the comparison of ECT versus sham-ECT for treatment-resistant schizophrenia, we identified one study with a high risk of bias and a very small sample size $(n=25)$ (Summary of findings for the main comparison). Very low- to low-quality evidence showed no clear short-term difference between groups in mental state and that there were fewer readmitted participants in the ECT group (three participants) than in the sham-ECT group (seven 
participants). No evidence was available on cognitive functioning or other predefined secondary outcomes.

\section{COMPARISON 2: ECT plus standard care versus antipsychotics (clozapine) plus standard care}

For the comparison of ECT versus antipsychotics (clozapine), there was no clear difference in the medium-term response to treatment based on low-quality evidence. Furthermore, very lowquality evidence was obtained from a study with a high risk of bias and a small sample size $(n=162)$ (Summary of findings 2). This study showed that ECT may be associated with both shortand medium-term improvement in patients' mental state (assessed by BPRS score). There were no clear differences in the incidence of adverse events between the groups, although participants who received ECT had lower medium-term TESS scores than participants who received clozapine. No evidence was available for cognitive functioning, satisfaction and acceptability of treatment, general functioning, and service outcomes.

\section{COMPARISON 3: ECT plus standard care versus standard care}

For the comparison of ECT versus standard care, data indicated that there more participants with a clinically important response to treatment in the ECT than in the standard care group. The quality of this evidence was moderate for the medium-term outcome (Summary of findings 3). Data showed that ECT may be associated with an improvement in patients' general mental state and some specific mental symptoms (such as anxiety and depression, lack of vitality, thought disturbance, agitation, hostile suspiciousness, psychosis, excitement, and hostility) regardless of whether shortor medium-term endpoints were considered. Electroconvulsive therapy was also associated with medium-term improvement in general functioning, but this evidence was obtained from limited data (Chanpattana 1999, $\mathrm{n}=30$; Jiang 2009, $\mathrm{n}=67$ ) at high risk of bias. For total adverse events, data showed lower mediumterm TESS scores in the ECT group than in the non-ECT group. $E C T$ increased the risk of memory deterioration, but no differences were observed for the risk of breathing discomfort and insomnia. There was no clear differences between groups for other outcomes including satisfaction and acceptability of treatment (leaving the study early), and most of the other adverse events. No evidence was available on cognitive functioning and service outcomes.

\section{COMPARISON 4: ECT alone versus antipsychotics (flupenthixol) alone}

For the comparison of ECT alone versus antipsychotics (flupenthixol) alone, we identified one study at high risk of bias with a very small sample size $(n=30)$ (Summary of findings 4$)$. Very lowquality evidence showed no clear differences in mental state and general functioning between groups. No evidence was available for our primary outcomes or the remaining secondary outcomes.

The other comparisons predefined in the protocol were not available in this review.

\section{Overall completeness and applicability of evidence}

Overall, the evidence is incomplete related to several aspects including participants, interventions, and outcomes. All included participants were between 18 and 46 years old (on average), with strictly diagnosed schizophrenia by international standards (ICD-10, DSM-IV, CCMD-2-R, and CCMD-3), and were well-defined as treatment-resistant in each included study. Most participants
(1161/1285, 90.6\%) were recruited from China, hence limiting the applicability of the findings for an illness that affects people worldwide.

In terms of interventions, all included studies used ECT plus standard care, with only one arm in one study ECT as sole intervention. Except for the comparison with placebo (no treatment), the comparators of sham-ECT, antipsychotics, or non-pharmacological forms of treatment were limited. Evidence for comparing different modalities of ECT (including electrode placement, duration of course, frequency of treatment) was also lacking.

Evidence for clinically important change in cognitive functioning (one of our primary outcomes) was not available; only data for memory deterioration were reported. There was a lack of data for most of our secondary outcomes, which is reflected in the 'Summary of findings' tables, namely acceptability of treatment (leaving the study early), mental state (clinically important change in general mental state), general functioning (clinically important change in general functioning), service outcomes (hospitalisation), and death. This may further limit the applicability of our findings.

\section{Quality of the evidence}

Overall, the quality of the evidence was very low to moderate, downgraded due to study limitations such as high risk of performance bias or imprecision. All the included studies except Goswami 2003 had a high risk of performance bias due to insufficient reporting of blinding of participants and personnel. Most studies did not clearly describe the method of random sequence generation and allocation concealment, and provided insufficient information on blinding of outcome assessment. Four studies that reported on attrition (less than 20\%) had an unclear risk of attrition bias. Two studies that did not report the primary outcome appropriately had a high risk of reporting bias. For the moderate-quality evidence on response to treatment, we are moderately confident in the effect estimate; the true effect is likely to be close to the estimate of effect, but the possibility exists that it is substantially different. For the very low- and low-quality evidence, we have very little or limited confidence in the effect estimate.

The other two main limitations of the body of evidence were indirectness and imprecision. Imprecision was due mainly to small sample size, low event rate, or wide confidence interval. Indirectness is relatively more prevalent in subjectively measured outcomes such as mental state and functioning, as usually numerical scales are employed as an indirect index.

\section{Potential biases in the review process}

We developed comprehensive search strategies and performed the search with no limitations on language, date, document type, or publication status. However, we only included published data, so there is a possibility of publication bias. Nonetheless, two review authors independently screened studies and extracted data, therefore it is less likely that this process could have introduced bias. 


\section{Agreements and disagreements with other studies or reviews}

This is an update of a previous Cochrane Review that was originally published in 2005 (Other published versions of this review). The previous review did not look specifically at the evidence for ECT in people with treatment-resistant schizophrenia. This updated review assessed the current evidence for ECT specifically for the group of people whose illness has been designated as resistant to treatment, therefore we excluded studies included in the previous version of the review that did not state that the participants were diagnosed as treatment-resistant (Other published versions of this review). There is another systematic review assessing the efficacy and safety of ECT in combination with antipsychotics (except for clozapine) versus the same antipsychotic monotherapy for treatment-resistant schizophrenia (Zheng 2016). Zheng 2016 found that ECT was superior to antipsychotic monotherapy in terms of clinical response, symptomatic improvement, and specific mental symptoms based on the PANSS score. The present review did not predefine the exact same outcomes as Zheng 2016, but reached a similar conclusion.

\section{AUTHORS' CONCLUSIONS}

\section{Implications for practice}

\section{For people with treatment-resistant schizophrenia}

When ECT plus standard care was compared with standard care, moderate-quality evidence showed medium-term benefits of ECT in terms of response to treatment. There was some advantage of ECT on mental state and general functioning. However, ECT may also induce memory deterioration. The quality of this evidence was very low or low, indicating limited confidence in the effect estimate. This may change with future research. There was also a lack of evidence evaluating ECT versus other controls (sham-ECT or other antipsychotics). Additionally, there is insufficient evidence regarding the long-term effects or safety parameters of ECT to enable a more comprehensive evaluation.

\section{For clinicians}

When combined with standard care, ECT may improve patients' clinical response, mental state, and general functioning when compared with antipsychotics alone. However, the current evidence is too weak to indicate whether ECT is superior or inferior to other controls for the management of people with treatmentresistant schizophrenia.

\section{For policymakers and funders}

Very low- to moderate-quality evidence indicates that there are some benefits of adding ECT to standard care for treatmentresistant schizophrenia. However, very low-quality evidence shows that ECT may cause specific adverse events (such as memory deterioration). In summary, there is a lack of evidence from this review to either support or discourage the use of ECT as an add-on treatment. There is a need for further rigorous, well-designed, and large trials in more countries.

\section{Implications for research}

\section{General}

Strict adherence to the CONSORT recommendations for reporting of trials is suggested (Pandis 2017). Generation of the allocation sequence, allocation concealment, and blinding are important parts of methodology and should be well-reported. We also suggest that the availability of all data should be expanded (not only shown in figures or transformed for skewed data). This would prevent valuable data or trials from being excluded in a meta-analysis, and would avoid redundancy (multiple publications) and facilitate the production of clearer results.

\section{Specific}

Further studies with high-quality methodology should address the following areas:

- ensuring blinding of participants, personnel, and outcome assessor;

- ensuring an $80 \%$ statistical power for primary outcome (sufficient sample size);

- exploring the use of ECT, or the different forms of ECT (unilateral versus bilateral, long course versus short course, continuation versus maintenance);

- focusing on key outcomes in this review;

- extending the follow-up duration to longer than six months.

\section{A C K N O WLEDGEMENTS}

The Cochrane Schizophrenia Group Editorial Base in Nottingham produces and maintains standard text for use in the Methods section of their reviews. We have used this text as the basis of what appears here and adapted it as required.

The search term was developed by the Information Specialist of the Cochrane Schizophrenia Group and the contact author of this protocol.

Prathap Tharyan conducted a review of ECT for schizophrenia, on which this review was built, looking specifically at people with treatment-resistant schizophrenia.

We would like to thank Elizabeth Cummings for her peer review of the protocol as well as Genevieve Gariepy, Rawan Al Khudari and Vivek Agarwal for their peer review of the review version. 


\section{REFERE N CE S}

\section{References to studies included in this review}

Cai 2008 \{published data only\}

Cai XY, Liang JL, Su Y. A comparison study of MECT combining clozapine in the treatment of treatment-resistant schizophrenia [无抽搐电休克合并氯氮平治疗难治性精神分裂症的对照 研究] . \#\#\#\#\# [Medical Journal of Chinese People's Health] 2008;20(13):1423-4. [CSzG: Ref17951]

\section{Chanpattana 1999 \{published data only\}}

Chanpattana W. ECT in schizophrenia. Journal of the Psychiatric Association of Thailand 1999;44(2):156-70. [CSzG: Ref8163]

Chanpattana W, Chakrabhand ML, Kitaroonchai W, Choovanichvong S, Prasertsuk Y. Effects of twice- versus thriceweekly electroconvulsive therapy in schizophrenia. Journal of the Medical Association of Thailand 1999;82(5):477-82.

* Chanpattana W, Chakrabhand ML, Sackeim HA, Kitaroonchai W, Kongsakon R, Techakasem P, et al. Continuation ECT in treatment resistant schizophrenia: a controlled study. Journal of ECT 1999;15(3):178-92. [CSzG: Ref4913; MEDLINE: 10492856]

Chen 2012 \{published data only\}

Chen DR. Controlled study on MECT for treatment-resistant schizophrenia [无抽搐电休克治疗难治性精神分裂症的对照 分析] . \#\#\#\# [Xinjiang Medical Journal] 2012;9:57-60. [CSzG: Ref32661]

\section{Goswami 2003 \{published data only\}}

Goswami U, Kumar U, Singh B. Efficacy of electroconvulsive therapy in treatment resistant schizophrenia syndrome: a double-blind study. Indian Journal of Psychiatry 2001; Vol. 43. [CSzG: Ref14832]

* Goswami U, Kumar U, Singh B. Efficacy of electroconvulsive therapy in treatment resistant schizophrenia: a double-blind study. Indian Journal of Psychiatry 2003;45(1):26-9. [CSzG: Ref14826]

\section{Jiang 2009 \{published data only\}}

Jiang XQ, Yang KR, Zhou B, Jin P, Zheng LF, Gao XF, et al. Study on efficacy of modified electroconvulsive therapy (MECT) together with risperidone in treatment-resistant schizophrenia (TRS) [利培酮合并无抽搐电休克治疗难治性精神分裂症的临 床研究]. Chinese Journal of Nervous and Mental Disorders [\#\#\# \#\#\#\#\#] 2009;35(2):79-83. [CSzG: Ref22088]

\section{Jiang 2013 \{published data only\}}

Jiang ZW. Clinical analysis on MECT combined with ziprasidone in treatment of patients with refractory schizophrenia [无抽 搐电休克联合齐拉西酮治疗难治性精神分裂症临床效果分 析] . Journal of Clinical and Experimental Medicine [\#\#\#\#\#\#\#] 2013;17:1394-5. [CSzG: Ref27359]

\section{Lin 2014 \{published data only\}}

Lin K, Chen XM. Clinical effect on MECT combined with ziprasidone in treatment of patients with refractory schizophrenia [无抽搐电休克联合齐拉西酮治疗难治性精神分 裂症临床效果刍议] . Yiayao Qianyan [\#\#\#\#] 2014;19:202-3.

\section{Liu 2010 \{published data only\}}

Liu FQ, Gong GQ. The effect of MECT for treatment-resistant schizophrenic patients [精神分裂症患者无抽搐电休克的效 果] . China Modern Doctor [\#\#\#\#\#\#] 2010;48(1):29, 36. [CSzG: Ref22012]

\section{Petrides 2015 \{published data only\}}

Braga RJ, Mendelowitz AJ, Fink M, Schooler NR, Bailine SH, Malur $C$, et al. A randomized controlled trial of ECT in clozapine-refractory schizophrenia. Biological Psychiatry 2009;65(8):212S-3S. [CSzG: Ref19511]

Petrides G. ECT augmentation in clozapine-resistant schizophrenia: acute efficacy and maintance data. Schizophrenia Bulletin 2015;41:S328. [CSzG: Ref29626]

Petrides G. Electroconvulsive therapy (ECT) for medication resistant schizophrenia. Schizophrenia Research 2012;136:S22. [CSzG: Ref29625]

Petrides G, Goldberg T, John M, Braga R, Malur C, Malhotra A, et al. Electroconvulsive therapy for clozapine-resistant schizophrenia: cognitive effects. Neuropsychopharmacology 2016;41:S257. [CSzG: Ref35678]

* Petrides G, Malur C, Braga RJ, Bailine SH, Schooler NR, Malhotra AK, et al. Electroconvulsive therapy augmentation in clozapine-resistant schizophrenia: a prospective, randomized study. American Journal of Psychiatry 2015;172(1):52-8. [CSzG: Ref29366]

\section{Wang 2008 \{published data only\}}

Wang BH, Zeng DZ, Fan XW, Hua SG. Efficacy of modified electroconvulsive therapy in treatment-refractory schizophrenia [无抽搐电休克对难治性精神分裂症的增效作用]. \#\#\#\# \#\#\# [Journal of Clinical Psychiatry] 2008;18(6):415-7. [CSzG: Ref17865]

\section{Wang 2011 \{published data only\}}

Wang ZH, Ling YX, Xia Z. The role of modified ECT treatment of refractory schizophrenia [改良电抽搐治疗难治性精神分裂症 的作用] . Journal of Clinical Psychological Medicine [\#\#\#\#\#\#] 2011;21(4):252-4. [CSzG: Ref23979]

\section{Wang 2013 \{published data only\}}

Wang F, Guo DW. The effect on olanzapine combined with modified electroconvulsive therapy in refractory schizophrenia [奥氮平联合改良电休克治疗难治性精神分裂症的疗效观 察] . Clinical Rational Drug Use [\#\#\#\#\#\#\#] 2013;24:99. [CSzG: Ref27406]

\section{Yang 2005 \{published data only\}} Yang KJ, Liu TB, Yang HC. The effect of clozapine combining MECT for the resistant schizophrenics with agitation and aggressive behavior. Medical Journal of Chinese People's Health [\#\#\#\#\#] 2005;17(9):485-6. [CSzG: Ref12755] 
Zhang 2010 \{published data only\}

Zhang YJ, Liu Q, Hu Y, Zhou LG. Effects of modified electroconvulsive therapy combined with clozapine in patients with treatment-refractory schizophrenia [无抽搐电痉挛治疗 合并氯氮平治疗难治性精神分裂症及其对记忆力的影响]. Chinese Mental Health Journal [\#\#\#\#\#\#] 2010;24(6):440-4. [CSzG: Ref22996]

\section{Zhang 2012 \{published data only\}}

Zhang SR, Zhang JL, Li XZ, Yang YP, Lang SY. The effect of MECT combined with olanzapine for refractory schizophrenia [无抽搐 电休克联合奥氮平治疗难治性精神分裂症疗效观察]. People's Military Surgeon [\#\#\#\#] 2012;2:141-3. [CSzG: Ref27071]

\section{References to studies excluded from this review}

Abraham 1987 \{published data only\}

Abraham KR, Kulhara P. The efficacy of electroconvulsive therapy in the treatment of schizophrenia. A comparative study. British Journal of Psychiatry 1987;151:152-5. [MEDLINE: 3318990]

\section{Abrams 1967 \{published data only\}}

Abrams R. Daily administration of unilateral ECT. American Journal of Psychiatry 1967;124(3):384-6. [MEDLINE: 6039994]

\section{Agarwal 1985 \{published data only\}}

Agarwal AK, Winny GC. Role of ECT phenothiazine combination in schizophrenia. Indian Journal of Psychiatry 1985;27(3):233-6.

\section{Arato 1980 \{published data only\}}

Arato M, Erdos A, Kurcz M, Vermes I, Fekete M. Studies on the prolactin response induced by electroconvulsive therapy in schizophrenics. Acta Psychiatrica Scandinavica 1980;61(3):239-44. [MEDLINE: 7376927]

\section{Bagadia 1981 \{published data only\}}

Bagadia VN, Abhyankar RR, Doshi J, Pradhan PV, Shah LP. A double blind controlled study of ECT vs chlorpromazine in schizophrenia. Journal of the Association of Physicians of India 1983;31(10):637-40. [MEDLINE: 6671932]

Bagadia VN, Abhyankar RR, Doshi J, Pradhan PV, Shah LP. Re-evaluation of ECT in schizophrenia. Psychopharmacology Bulletin 1983;19(3):550-5.

Bagadia VN, Shah LP, Pradhan PV, Doshi J, Abhyankar RR. Evaluation of cognitive effects of ECT: preliminary observations. Indian Journal of Psychiatry 1981;23(4):324-9.

\section{Bagadia 1988 \{published data only\}}

Bagadia VN, Abhyankar RR, Pradhan PV, Shah LP. Reevaluation of ECT in schizophrenia: right temporoparietal versus bitemporal electrode placement. Convulsive Therapy 1988;4:215-20

\section{Baker 1958 \{published data only\}}

Baker AA, Game JA, Thorpe JG. Physical treatment for schizophrenia. Journal of Mental Science 1958;104:860-4.
Thorpe JG, Baker AA. The effects of physical treatment on some psychological functions. Journal of Mental Science 1958;104:865-9.

Baker 1960 \{published data only\}

Baker AA, Bird G, Lavin NI, Thorpe JG. ECT in schizophrenia. Journal of Mental Science 1960;106:1506-11.

Baker 1960a \{published data only\}

Baker AA, Game JA, Thorpe JG. Some research into the treatment of schizophrenia in the mental hospital. Journal of Mental Science 1960;106:203-13.

Bhatia 1987 \{published data only\}

Bhatia MS, Balkrishna, Dhar NK, Bohra N. Schizophrenia: electrolyte profile \& the effect of treatment. Indian Journal of Psychiatry 1987;29(3):275-7.

Brandon 1985 \{published data only\}

Brandon S, Cowley P, McDonald C, Neville P, Palmer R, Wellstood ES. Leicester ECT trial: results in schizophrenia. British Journal of Psychiatry 1985;146:177-83. [MEDLINE: 3884080]

Brill 1959 \{published data only\}

Brill NQ, Crumpton E, Eiduson S, Grayson HM, Hellman LI. Predictive and concomitant variables related to improvement with actual and simulated ECT. Archives of General Psychiatry 1959;1:263-72.

Brill NQ, Crumpton E, Eiduson S, Grayson HM, Hellman LI, Richards RA. An experimental study of the relative effectiveness of various components of electro-convulsive therapy. American Journal of Psychiatry 1959;115:734-5.

Brill NQ, Crumpton E, Eiduson S, Grayson HM, Hellman LI, Richards RA. Relative effectiveness of various components of electroconvulsive therapy. Archives of Neurology and Psychiatry 1959;81:627-35.

Brill NQ, Crumpton E, Eiduson S, Grayson HM, Hellman LI, Richards RA, et al. Investigation of the therapeutic components and various factors associated with improvement with electroconvulsive treatment: a preliminary report. American Journal of Psychiatry 1957;113(11):997-1008. [MEDLINE: 13411281]

Eiduson S, Brill NG, Crumpton E. The effect of electro-convulsive therapy on spinal fluid constituents. Journal of Mental Science 1960;106:692-8.

\section{Chatterjee 1980 \{published data only\}}

Chatterjee SB, Mohammed E. Evaluation of unilateral electroconvulsive therapy (a double blind study). Indian Journal of Psychiatry 1980;22(2):185-94.

\section{ChiCTR-OPC-14005339 \{published data only\}}

ChiCTR-OPC-14005339. The study of the brain network features in refractory schizophrenia. apps.who.int/trialsearch/ Trial2.aspx?TrialID=ChiCTR-OPC-14005339 (first received 16 October 2014). 
d'Elia 1970 \{published data only\}

d'Elia G. Comparison of ECT with unilateral and bilateral stimulation. IV. Retrograde amnesia. Acta Psychiatrica Scandinavica Supplementum 1970;215:61-75.

d'Elia G. Comparison of ECT with unilateral and bilateral stimulation. V. Confusion and other side-effects. Acta Psychiatrica Scandinavica Supplementum 1970;215:76-89.

d'Elia G, Perris C. Comparison of ECT with unilateral and bilateral stimulation: I. Seizure and post-seizure electroencephalographic pattern. Acta Psychiatrica Scandinavica Supplementum 1970;215:9-29.

\section{Doongaji 1973 \{published data only\}}

Doongaji DR, Jeste DV, Saoji NJ, Kane PV, Ravindranath S. Unilateral versus bilateral ECT in schizophrenia. British Journal of Psychiatry 1973;123(572):73-9. [MEDLINE: 4729877]

\section{El Islam 1970 \{published data only\}}

El Islam MF, Ahmed SA, Erfan ME. The effect of unilateral ECT on schizophrenic delusions and hallucinations. British Journal of Psychiatry 1970;117(539):447-8. [MEDLINE: 5481209]

\section{Gan 2017 \{published data only\}}

Gan JL, Duan HF, Cheng ZX, Yang JM, Zhu XQ, Gao CY, et al. Neuroprotective effect of modified electroconvulsive therapy for schizophrenia: a proton magnetic resonance spectroscopy study. Journal of Nervous and Mental Disease (in press).

Gander 1967 \{published data only\}

Gander DR, Bennett PJ, Kelly DH. Hexafluorodiethyl ether (Indoklon) convulsive therapy: a pilot study. British Journal of Psychiatry 1967;113(505):1413-8. [MEDLINE: 4383928]

\section{Gangadhar 2000 \{published data only\}}

Gangadhar B, Mayur P, Janakiramaiah N, Subbakrishna D, Rao G. Cardiovascular response during ECT: a cross-over study across stimulus conditions. Journal of ECT 2000;16(2):177-82.

\section{Girish 2003 \{published data only\}}

Girish K, Gill NS. Electroconvulsive therapy in lorazepam non-responsive catatonia. Indian Journal of Psychiatry 2003;45(1):21-5

Kunigiri G, Gill NS, B GN, Naimmagadda J. Electroconvulsive therapy in lorazepam non-responsive catatonia. Proceedings of the 12th World Congress of Psychiatry; 2002 Aug 24-29; Yokohama, Japan. 2002.

\section{Hargreaves 1972 \{published data only\}}

Hargreaves WA, Fischer A, Elashoff RM, Blacker KH. Delayed onset of impairment following electrically induced convulsions. Acta Psychiatrica Scandinavica 1972;48(1):69-77. [MEDLINE: 5027530]

\section{He 2001 \{published data only\}}

He Q, Liu BL, Qin QL. A comparative study of side effects of non- versus dominance ECT on schizophrenics with EEG and ECG monitoring. Acta Academiae Medicinae Shandong 2001;39(1):48-50.

\section{Heath 1964 \{published data only\}}

Heath ES, Adams A, Wakeling PLG. Short courses of ECT and simulated ECT in chronic schizophrenia. British Journal of Psychiatry 1964;110:800-7.

Janakiramaiah 1981 \{published data only\} Janakiramaiah N, Subbakrishna DK. ECT-chlorpromazine combination compared with chlorpromazine only in schizophrenia. Indian Journal of Psychiatry 1981;23:230-3.

Janakiramaiah 1982 \{published data only\}

Janakiramaiah N, Channabasavanna SM, Narashimha Murthy NS. ECT-chlorpromazine combination versus chlorpromazine alone in acutely schizophrenic patients. Acta Psychiatrica Scandinavica 1982;66(6):464-70. [MEDLINE: 7180565]

Krystal 1993 \{published data only\}

Krystal AD, Weiner RD, McCall WV, Shelp FE, Arias R, Smith P. The effects of ECT stimulus dose and electrode placement on the ictal electroencephalogram: an intraindividual crossover study. Biological Psychiatry 1993;34(11):759-67. [MEDLINE: 8292679]

\section{Laurell 1970 \{published data only\}}

Laurell B. Comparison of electric and flurothyl convulsive therapy: confusion and other side effects. Acta Psychiatrica Scandinavica Supplementum 1970;213:61-73.

Laurell B. Comparison of electric and flurothyl convulsive therapy: retrograde amnesia. Acta Psychiatrica Scandinavica Supplementum 1970;213:51-60.

Laurell 1970a \{published data only\}

Laurell B, Perris C. Comparison of ECT and ICT. I. Seizure and post-seizure electroencephalographic pattern. Acta Psychiatrica Scandinavica Supplementum 1970;213:8-21.

Marjerrison 1975 \{published data only\} Marjerrison G, James J, Reichert H. Unilateral and bilateral ECT: EEG findings. Canadian Psychiatric Association Journal 1975;20(4):257-66. [MEDLINE: 1182637]

\section{Miller 1953 \{published data only\}}

Miller DH, Clancy J, Cumming E. A comparison between unidirectional current nonconvulsive electrical stimulation given with Reiter's machine, standard alternating current electroshock (Cerletti method), and pentothal in chronic schizophrenia. American Journal of Psychiatry 1953;109:617-20.

\section{Naid oo 1956 \{published data only\}}

Naidoo D. The effects of reserpine (serpasil) on the chronic disturbed schizophrenic: a comparative study of rauwolfia alkaloids and electroconvulsive therapy. Abstracts of World Medicine 1957;21(2):133.

Naidoo D. The effects of reserpine (serpasil) on the chronic disturbed schizophrenic: a comparative study of rauwolfia alkaloids and electroconvulsive therapy. Journal of Nervous and Mental Disease 1956;123:1-13. 
Natani 1983 \{published data only\}

Natani GD, Gautam S, Gehlot PS. Comparison of three treatment regimes in schizophrenia. Indian Journal of Psychiatry 1983;25(4):306-11.

\section{NCT02159001 \{published data only\}}

NCT02159001. Efficacy and cognitive effects of electroconvulsive therapy in clozapine-resistant schizophrenia. clinicaltrials.gov/show/NCT02159001 (first received 4 June 2014).

\section{Rami 2008 \{published data only\}}

Rami L, Goti J, Ferrer J, Marcos T, Salamero M, Bernardo M. Cognitive functions after only one ECT session: a controlled study. Psychiatry Research 2008;158(3):389-94. [MEDLINE: 18279973]

\section{Reichert 1976 \{published data only\}}

Reichert H, Benjamin J, Keegan D, Marjerrison G. Bilateral and non dominant unilateral ECT: I. Therapeutic efficacy. Canadian Psychiatric Association Journal 1976;21(2):69-78.

\section{Sarkar 1994 \{published data only\}}

Sarkar P, Andrade C, Kapur B, Das P, Sivaramakrishna Y, Harihar C, et al. An exploratory evaluation of ECT in haloperidoltreated DSM-IIIR schizophreniform disorder. Convulsive Therapy 1994;10(4):271-8. [MEDLINE: 7850397]

\section{Small 1968 \{published data only\}}

Small JG, Small IF, Sharpley P, Moore DF. A double-blind comparative evaluation of flurothyl and ECT. Archives of General Psychiatry 1968;19(1):79-86. [MEDLINE: 5658383]

\section{Smith 1967 \{published data only\}}

Smith K, Surphlis WRP, Gynther MD, Shimkunas AM. ECTchlorpromazine and chlorpromazine compared in the treatment of schizophrenia. Journal of Nervous and Mental Disease 1967;144:284-90.

\section{Stenback 1957 \{published data only\}}

Stenback A, Viitamaki RO, Kukkonen S. Personality changes in electroconvulsive treatment. Acta Psychiatrica et Neurologica Scandinavica 1957;32:345-59.

\section{Swoboda 2001 \{published data only\}}

Swoboda E, Conca A, Konig P, Waanders R, Hansen M. Maintenance electroconvulsive therapy in affective and schizoaffective disorder. Neuropsychobiology 2001;43(1):23-8. [MEDLINE: 11150895]

\section{Taylor 1980 \{published data only\}}

Taylor P. ECT and schizophrenia in Great Britain. American Journal of Psychiatry 1981;138(2):259-60. [MEDLINE: 7006422]

Taylor P, Fleminger JJ. ECT for schizophrenia. Lancet 1980;1(8183):1380-3. [MEDLINE: 6104172]

Taylor PJ. Schizophrenia and ECT: a case for change in prescription?. Dilemmas and Difficulties in the Management of Psychiatric Patients. Oxford, UK: Oxford University Press, 1990:143-55

\section{Ukpong 2002 \{published data only\}}

Ukpong DI, Makanjuola ROA, Morakinyo O. A controlled trial of modified electroconvulsive therapy in schizophrenia in a Nigerian teaching hospital. West African Journal of Medicine 2002;21(3):237-40. [MEDLINE: 12744577]

Ulett 1956 \{published data only\}

Ulett GA, Smith K, Gleser GC. Evaluation of convulsive and subconvulsive shock therapies utilizing a control group. American Journal of Psychiatry 1956;112:795-802.

\section{Ungvari 1981 \{published data only\}}

Ungvari G, Bitter I, Czobor P, Vitrai J, Pethoe B. The role of high-dosage neuroleptic therapy and electroconvulsive therapy in the treatment of the acute phase of schizophrenia [Zur Rolle der neuroleptischen Hochdosis-Therapie und des Elektroschocks bei der Behandlung der akuten Phase von Schizophrenien]. Psychiatrie, Neurologie Und Medizinische Psychologie 1981;33(8):458-63. [MEDLINE: 6124987]

Ungvari G, Petho B. High-dose haloperidol therapy: its effectiveness and a comparison with electroconvulsive treatment. Journal of Psychiatric Treatment and Evaluation 1982;4:279-83.

\section{Wessels 1971 \{published data only\}}

Wessels WH. A comparative study of the efficacy of bilateral and unilateral ECT with thioridazine in acute schizophrenia. Proceedings of the 5th World Congress of Psychiatry; 1971 Nov 28 - Dec 4; Mexico City, Mexico. 1971:574.

Wessels WH. A comparative study of the efficacy of bilateral and unilateral electroconvulsive therapy with thioridazine in acute schizophrenia. South African Medical Journal 1972;46(26):890-2.

Xue 1985 \{published data only\}

Xue CC, Xie H, Ruan Q, Cheng Y, Luo D. Electric acupuncture convulsive therapy. Convulsive Therapy 1985;1(4):242-51.

Zadeh 2006 \{published data only\}

Zadeh MA. Comparison effects of clozapine, ECT and combination ECT and clozapine on resistance treatment of schizophrenia (double blind). Journal of Mazandaran University of Medical Sciences 2006;50(15):14-7.

Zhou 2003 \{published data only\}

Zhou XD, Zhang DZ, Wang ZT. The effect of modern ECT on hypothalamic concentration of coricotropin and thyrotropin releasing hormones in patients with schizophrenia. Medical Journal of National Defending Forces in North China 2003;15(6):402-3.

\section{References to studies awaiting assessment}

Chen 2006 \{published data only\}

Chen M. Observation and nursing of multi-parameter monitoring electroconvulsive therapy for schizophrenia [多参 数监护电休克治疗精神分裂症的疗效观察及护理]. 中国现代 临床护理学杂志 [Journal of Chinese Modern Clinical Nursing] 2006; Vol. 1, issue 5:433. 


\section{Melzer 2015 \{published data only\}}

Melzer-Ribeiro DL, Rigonatti SP, Kayo M, Ribeiro RB, Avrichir B, Fortes $\mathrm{M}$, et al. Pilot double blind, placebo controlled and randomized study to assess electroconvulsive therapy efficacy as augmenting strategy to clozapine in super-refractory schizophrenia. Schizophrenia Bulletin 2015;41:S323-4.

NCT02049021. Electroconvulsive therapy (ECT) in patients with super refractory schizophrenia [Pilot double blind, placebo controlled and randomized study to assess electroconvulsive therapy efficacy as augmenting strategy to clozapine in superrefractory schizophrenia]. clinicaltrials.gov/show/NCT02049021 (first received 15 January 2014).

\section{Nicholas 1996 \{published data only\}}

Nicholas JD, Jame SL, Martin R, Rita MK, James I, Dennis WL, et al. Electrical dose and seizure threshold in bifrontal, bitemporal and right unilateral ECT - relations to clinical outcome and cognitive. Proceedings of the 149th Annual Meeting of the American Psychiatric Association; 1996 May 4-9; New York, USA. 1996.

\section{Oleneva 2005 \{published data only\}}

Oleneva E, Tsukarzi E, Mosolov S. Combined olanzapineECT therapy for resistant schizophrenia. European Neuropsychopharmacology 2005;15(Suppl 2):S131.

Oleneva E, Tsukarzi E, Mosolov SN. Combined olanzapine-ECT therapy for resistant schizophrenia. International Journal of Neuropsychopharmacology 2008;11(Suppl 1):151.

\section{Peyman 1956 \{published data only\}}

Peyman DAR. An investigation of the effects of group psychotherapy on chronic schizophrenic patients. Group Psychotherapy 1956;9:35-9.

\section{Sackeim 2000 \{published data only\}}

Sackeim HA. Affective and cognitive consequences of ECT. CRISP Database (https://www-commons.cit.nih.gov/crisp/ index.html. National Research Register, (accessed 19 February 2001).

\section{References to ongoing studies}

\section{NCT00042224 \{published data only\}}

NCT00042224. ECT in clozapine refractory schizophrenia. www.clinicaltrials.gov/ct/show/NCT00042224 (first received 24 July 2002).

\section{NCT00753051 \{published data only\}}

NCT00753051. Treat clozapine-resistant schizophrenia comparing between clozapine + haloperidol versus clozapine + electroconvulsive therapy (ECT). www.clinicaltrials.gov/ct/ show/NCT00753051 (first received 15 September 2008).

\section{NCT02926976 \{published data only\}}

NCT02926976. The optimal treatment for treatment-resistant schizophrenia. www.clinicaltrials.gov/show/NCT02926976 (first received 5 October 2016).

\section{Additional references}

\section{Agarwal 1992}

Agarwal AK, Andrade C, Reddy MV. The practice of ECT in India: issue relating to the administration of ECT. Indian Journal of Psychiatry 1992;34(4):285-97.

\section{Altman 1996}

Altman DG, Bland JM. Detecting skewness from summary information. BMJ 1996;313(7066):1200.

\section{APA 2008}

American Psychiatric Association. The Practice of Electroconvulsive Therapy: Recommendations for Treatment, Training, and Privileging (A Task Force Report of the American Psychiatric Association). Arlington, VA: American Psychiatric Association Publishing, 2008.

\section{Bland 1997}

Bland JM, Kerry SM. Statistics notes: trials randomised in clusters. BMJ 1997;315(7108):600.

\section{Bocchio-Chiavetto 2006}

Bocchio-Chiavetto L, Zanardini R, Bortolomasi M, Abate M, Segala M, Giacopuzzi M, et al. Electroconvulsive therapy (ECT) increases serum brain derived neurotrophic factor (BDNF) in drug resistant depressed patients. European Neuropsychopharmacology 2006;16(8):620-4.

\section{Boissel 1999}

Boissel JP, Cucherat M, Li W, Chatellier G, Gueyffier F, Buyse $M$, et al. The problem of therapeutic efficacy indices. 3. Comparison of the indices and their use [Apercu sur la problematique des indices d'efficacite therapeutique, 3 : comparaison des indices et utilisation. Groupe d'Etude des Indices D'efficacite]. Therapie 1999;54(4):405-11. [PUBMED: 10667106]

\section{Carpenter 2000}

Carpenter WT Jr, Gold JM, Lahti AC, Queern CA, Conley RR, Bartko JJ, et al. Decisional capacity for informed consent in schizophrenia research. Archives of General Psychiatry 2000;57(6):533-8.

\section{Conley 1997}

Conley RR, Buchanan RW. Evaluation of treatment-resistant schizophrenia. Schizophrenia Bulletin 1997;23(4):663-74.

\section{Deeks 2000}

Deeks J. Issues in the selection for meta-analyses of binary data. Proceedings of the 8th International Cochrane Colloquium. Cape Town. The Cochrane Collaboration, 25-28 Oct 2000.

\section{Devanand 1994}

Devanand DP, Dwork AJ, Hutchinson ER, Bolwig TG, Sackeim HA. Does ECT alter brain structure?. American Journal of Psychiatry 1994;151(7):957-70. 


\section{Divine 1992}

Divine GW, Brown JT, Frazier LM. The unit of analysis error in studies about physicians' patient care behavior. Journal of General Internal Medicine 1992;7(6):623-9.

\section{Donner 2002}

Donner A, Klar N. Issues in the meta-analysis of cluster randomized trials. Statistics in Medicine 2002;21:2971-80.

\section{Dwork 2004}

Dwork AJ, Arango V, Underwood M, Ilievski B, Rosoklija G, Sackeim HA, et al. Absence of histological lesions in primate models of ECT and magnetic seizure therapy. American Journal of Psychiatry 2004;161(3):576-8. [PUBMED: 14992989]

\section{Egger 1997}

Egger M, Davey Smith G, Schneider M, Minder C. Bias in meta-analysis detected by a simple, graphical test. $B M J$ 1997;315(7109):629-34.

\section{Elbourne 2002}

Elbourne D, Altman DG, Higgins JPT, Curtin F, Worthington HV, Vail A. Meta-analyses involving cross-over trials: methodological issues. International Journal of Epidemiology 2002;31(1):140-9.

\section{Ende 2000}

Ende G, Braus DF, Walter S, Weber-Fahr W, Henn FA. The hippocampus in patients treated with electroconvulsive therapy: a proton magnetic resonance spectroscopic imaging study. Archives of General Psychiatry 2000;57(10):937-43.

\section{Endler 1988}

Endler NS. The origins of electroconvulsive therapy (ECT). Convulsive Therapy 1988;4(1):5-23.

\section{Furukawa 2006}

Furukawa TA, Barbui C, Cipriani A, Brambilla P, Watanabe N. Imputing missing standard deviations in meta-analyses can provide accurate results. Journal of Clinical Epidemiology 2006;59(7):7-10.

\section{Gangadhar 2010}

Gangadhar BN, Thirthalli J. Frequency of electroconvulsive therapy sessions in a course. Journal of ECT 2010;26(3):181-5.

\section{Gulliford 1999}

Gulliford MC, Ukoumunne OC, Chinn S. Components of variance and intraclass correlations for the design of communitybased surveys and intervention studies: data from the Health Survey for England 1994. American Journal of Epidemiology 1999;149(9):876-83.

\section{Harrison 2001}

Harrison G, Hopper K, Craig T, Laska E, Siegel C, Wanderling J, et al. Recovery from psychotic illness: a 15- and 25-year international follow-up study. British Journal of Psychiatry 2001;178:506-17.

\section{Higgins 2003}

Higgins JP, Thompson SG, Deeks JJ, Altman DG. Measuring inconsistency in meta-analyses. BMJ 2003;327(7414):557-60.

\section{Higgins 2011}

Higgins JPT, Green S (editors). Cochrane Handbook for Systematic Reviews of Interventions Version 5.1.0 (updated March 2011). The Cochrane Collaboration, 2011. Available from handbook.cochrane.org.

\section{Hutton 2009}

Hutton JL. Number needed to treat and number needed to harm are not the best way to report and assess the results of randomised clinical trials. British Journal of Haematology 2009;146(1):27-30.

\section{Kala 2013}

Kala A. Time to face new realities; mental health care bill - 2013. Indian Journal of Psychiatry 2013;55(3):216-9.

\section{Kane 1988}

Kane J, Honigfeld G, Singer J, Meltzer H. Clozapine for the treatment-resistant schizophrenic. A double-blind comparison with chlorpromazine. Archives of General Psychiatry 1988;45(9):789-96.

\section{Kay 1986}

Kay SR, Fiszbein A, Opfer LA. Positive and Negative Syndrome Scale (PANSS) Manual. North Tonawanda, NY: Multi-Health Systems, 1986.

\section{Kendell 1981}

Kendell RE. The present status of electroconvulsive therapy. British Journal of Psychiatry 1981;139:265-83.

\section{Leiknes 2012}

Leiknes KA, Jarosh-von Schweder L, Høie B. Contemporary use and practice of electroconvulsive therapy worldwide. Brain and Behavior 2012;2(3):283-344.

\section{Leon 2006}

Leon AC, Mallinckrodt CH, Chuang-Stein C, Archibald DG, Archer GE, Chartier K. Attrition in randomized controlled clinical trials: methodological issues in psychopharmacology. Biological Psychiatry 2006;59(11):1001-5. [PUBMED: 16905632]

\section{Leucht 2005}

Leucht S, Kane JM, Kissling W, Hamann J, Etschel E, Engel R. What does the PANSS mean?. Schizophrenia Research 2005; 79(2-3):231-8.

\section{Leucht 2005a}

Leucht S, Kane JM, Kissling W, Hamann J, Etschel E, Engel R. Clinical implications of Brief Psychiatric Rating Scale scores. British Journal of Psychiatry 2005;187:366-71.

\section{Lloyd 2011}

Lloyd K, White J. Democratizing clinical research. Nature 2011;474(7351):277-8.

\section{Loo 2010}

Loo C, Trollor J, Alonzo A, Rendina N, Kavess R. Mental health legislation and psychiatric treatments in NSW: electroconvulsive therapy and deep brain stimulation. Australasian Psychiatry 2010;18(5):417-25. [PUBMED: 20863180] 


\section{Madsen 2000}

Madsen TM, Treschow A, Bengzon J, Bolwig TG, Lindvall O, Tingström A. Increased neurogenesis in a model of electroconvulsive therapy. Biological Psychiatry 2000;47(12):1043-9.

\section{Marshall 2000}

Marshall M, Lockwood A, Bradley C, Adams C, Joy C, Fenton M. Unpublished rating scales: a major source of bias in randomised controlled trials of treatments for schizophrenia. British Journal of Psychiatry 2000;176:249-52.

\section{Marwaha 2004}

Marwaha S, Johnson S. Schizophrenia and employment - a review. Social Psychiatry and Psychiatric Epidemiology 2004;39(5):337-49. [PUBMED: 15133589]

\section{McGrath 2008}

McGrath J, Saha S, Chant D, Welham J. Schizophrenia: a concise overview of incidence, prevalence, and mortality. Epidemiologic Reviews 2008;30:67-76. [PUBMED: 18480098]

\section{Meltzer 1997}

Meltzer HY. Treatment-resistant schizophrenia - the role of clozapine. Current Medical Research and Opinion 1997;14(1):1-20.

\section{Monroe 1991}

Monroe RRJ. Maintenance electroconvulsive therapy. Psychiatric Clinics of North America 1991;14(4):947-60.

\section{Mughal 2013}

Mughal F, Menezes SB. Severe depression with Cotard's phenomenon: treatment of a capacitated patient within the United Kingdom's Mental Health Act 2007. Mental Illness 2013;5(1):e3. [PUBMED: 25478127]

\section{Muglia 2003}

Muglia P, Vicente AM, Verga M, King N, Macciardi F, Kennedy JL. Association between the BDNF gene and schizophrenia. Molecular Psychiatry 2003;8(2):146-7.

\section{NICE 2003}

National Institute for Health and Care Excellence. Guidance on the use of electroconvulsive therapy. NICE technology appraisal guidance [TA59]. Published date: April 2003. www.nice.org.uk/ guidance/ta59 (accessed prior to 5 February 2019).

\section{Nieto 2013}

Nieto R, Kukuljan M, Silva H. BDNF and schizophrenia: from neurodevelopment to neuronal plasticity, learning, and memory. Frontiers in Psychiatry 2013;4:45.

\section{Odejide 1987}

Odejide AO, Ohaeri JU, Ikuesan BA. Electroconvulsive therapy in Nigeria. Journal of ECT 1987;3(1):31-9.

\section{Overall 1962}

Overall JE, Gorham DR. The brief psychiatric rating scale. Psychological Reports 1962;10:799-812.

\section{Pandis 2017}

Pandis N, Chung B, Scherer RW, Elbourne D, Altman DG. CONSORT 2010 statement: extension checklist for reporting within person randomised trials. BMJ 2017;357:j2835.

\section{Rose 2003}

Rose D, Fleischmann P, Wykes T, Leese M, Bindman J. Patients' perspectives on electroconvulsive therapy: systematic review. BMJ 2003;326(7403):1363. [PUBMED: 12816822]

\section{Rosenquist 2014}

Rosenquist PB, Miller B, Pillai A. The antipsychotic effects of ECT: a review of possible mechanisms. Journal of ECT 2014;30(2):125-31.

\section{Sackeim 1993}

Sackeim HA, Prudic J, Devanand DP, Kiersky JE, Fitzsimons L, Moody BJ, et al. Effects of stimulus intensity and electrode placement on the efficacy and cognitive effects of electroconvulsive therapy. New England Journal of Medicine 1993;328(12):839-46.

\section{Sackeim 2000a}

Sackeim HA, Prudic J, Devanand DP, Nobler MS, Lisanby SH, Peyser S, et al. A prospective, randomized, double-blind comparison of bilateral and right unilateral electroconvulsive therapy at different stimulus intensities. Archives of General Psychiatry 2000;57(5):425-34.

\section{Scott 1991}

Scott AI, Weeks DJ, McDonald CF. Continuation electroconvulsive therapy: preliminary guidelines and an illustrative case report. British Journal of Psychiatry 1991;159:867-70.

\section{Scott 2005}

Scott A. The ECT Handbook: The Third Report of the Royal College of Psychiatrists' Special Committee of ECT. London: RCPsych Publications, 2005.

\section{Suzuki 2012}

Suzuki T, Remington G, Mulsant BH, Uchida H, Rajji TK, GraffGuerrero A, et al. Defining treatment-resistant schizophrenia and response to antipsychotics: a review and recommendation. Psychiatry Research 2012;197(1-2):1-6.

\section{Thompson 1994}

Thompson JW, Weiner RD, Myers CP. Use of ECT in the United States in 1975, 1980, and 1986. American Journal of Psychiatry 1994;151(11):1657-61.

\section{Ukoumunne 1999}

Ukoumunne OC, Gulliford MC, Chinn S, Sterne JA, Burney PG. Methods for evaluating area-wide and organisation-based interventions in health and health care: a systematic review. Health Technology Assessment 1999;3(5):iii-92.

\section{Weiner 1986}

Weiner RD, Rogers HJ, Davidson JRT, Squire LR. Effects of stimulus parameters on cognitive side effects. Annals of the New York Academy of Sciences 1986;462(1):315-25. 


\section{Weiner 1994}

Weiner RD. Treatment optimization with ECT.

Psychopharmacology Bulletin 1994;30(3):313-20.

\section{Xia 2009}

Xia J, Adams C, Bhagat N, Bhagat V, Bhoopathi P, El-Sayeh H, et al. Losing participants before the trial ends erodes credibility of findings. Psychiatric Bulletin 2009;33(7):254-7.

\section{Zheng 2016}

Zheng W, Cao XL, Ungvari GS, Xiang YQ, Guo T, Liu ZR, et al. Electroconvulsive therapy added to non-clozapine antipsychotic medication for treatment resistant schizophrenia: meta-analysis of randomized controlled trials. PLOS ONE 2016;11(6):e0156510.

\section{References to other published versions of this review}

\section{Tharyan 2005}

Tharyan P, Adams CE. Electroconvulsive therapy for schizophrenia. Cochrane Database of Systematic Reviews 2005, Issue 2. [DOI: 10.1002/14651858.CD000076.pub2; PUBMED: 15846598]

* Indicates the major publication for the study

\section{CHARACTERISTICS OF STUDIES}

Characteristics of included studies [ordered by study ID]

Cai 2008

Methods

Allocation: parallel, randomised

Blind: not stated

Setting: hospital (inpatient), single centre

Country: China

Length of study: 8 weeks

Participants

Diagnosis: treatment-resistant schizophrenia (CCMD-3)

Total $\mathbf{N}$ at randomisation $=100$

Sex: male 57 , female 43

Age (years): range 18 to 60

Length of illness (years): range 1 to 10

History: BPRS score > 50; received at least 3 types of resistance psychiatric drug therapy, of which at least 2 different classes of chemical structures; dose of each drug equivalent to chlorpromazine 600 $\mathrm{mg} / \mathrm{d}$ or more, failure after more than 4 weeks of treatment, or failure after 4 weeks of treatment when receiving clozapine ( $\geq 450 \mathrm{mg} / \mathrm{d}$ ) ('failure' was defined as the reduction rate of BPRS score $\leq 20 \%$ ).

Exclusion criteria: people with severe heart, liver, kidney disease, chronic body diseases, epilepsy, alcohol or drug abusers, MECT contraindications.

Interventions

ECT group $(n=50):$ MECT + clozapine

Content: participants who received clozapine before enrolment continued to use clozapine. Participants who received other antipsychotics before enrolment needed a 1-week wash-out period and then received clozapine. The initial dose of clozapine was $50 \mathrm{mg} / \mathrm{d}$, increased to 50 to $100 \mathrm{mg} / \mathrm{d}$ alternate days. The maximum dose of clozapine was no more than $300 \mathrm{mg} / \mathrm{d}$ when combined with MECT.

Frequency: 3 times a week for MECT, not stated for clozapine

Course of treatment (MECT): 6 to 12 sessions

Treatment duration: 8 weeks

Control group $(n=50)$ : Clozapine alone 
Cai 2008 (Continued)

Content: the usage and dosage of clozapine was same as above. The maximum dose ranged from 300 $\mathrm{mg} / \mathrm{d}$ to $700 \mathrm{mg} / \mathrm{d}$.

Frequency: not stated

Treatment duration: 8 weeks

Outcomes
Response to treatment: clinically significant response to treatment*
Mental state: assessed by BPRS
Adverse events: assessed by TESS
* ${ }^{*}$ Clinically significant response assessed by the reducing score rate of BPRS:
1) cure: reducing rate $\geq 75 \%$;
2) significant improvement: reducing rate between $50 \%$ and $74 \% ;$
3) improvement: reducing rate between $25 \%$ and $49 \% ;$
4) no clinical response: reducing rate $<25 \%$.
Clinically significant response to treatment was defined as cure + significant improvement.

Contact information: Third People's Hospital of Yuebei, Lechang, Guangdong, China

\section{Risk of bias}

\begin{tabular}{lll}
\hline Bias & Authors' judgement & Support for judgement \\
\hline $\begin{array}{l}\text { Random sequence genera- } \\
\text { tion (selection bias) }\end{array}$ & Unclear risk & Quote: "...were divided randomly into two groups..." (p.1423) \\
& & $\begin{array}{l}\text { Comments: The author described a random component in the sequence gen- } \\
\text { eration process, but no details were provided on randomisation method. }\end{array}$
\end{tabular}

\begin{tabular}{|c|c|c|}
\hline $\begin{array}{l}\text { Allocation concealment } \\
\text { (selection bias) }\end{array}$ & Unclear risk & $\begin{array}{l}\text { Comments: The author did not describe the allocation concealment. Insuffi- } \\
\text { cient information to permit judgement of 'low risk' or 'high risk'. }\end{array}$ \\
\hline
\end{tabular}

\begin{tabular}{|c|c|c|}
\hline $\begin{array}{l}\text { Blinding of participants } \\
\text { and personnel (perfor- }\end{array}$ & High risk & $\begin{array}{l}\text { Comments: Participants and personnel could not be blinded as } 1 \text { group did } \\
\text { not use ECT, and no sham-ECT was used. }\end{array}$ \\
\hline
\end{tabular}
mance bias)

All outcomes

Blinding of outcome as- Unclear risk sessment (detection bias) All outcomes
Comments: The author did not describe the blinding of outcome assessment. Insufficient information to permit judgement of 'low risk' or 'high risk'.

Incomplete outcome data Low risk Comments: No missing outcome data.
(attrition bias)
All outcomes

\begin{tabular}{lll}
\hline $\begin{array}{l}\text { Selective reporting (re- } \\
\text { porting bias) }\end{array}$ & Low risk & $\begin{array}{l}\text { Comments: The protocol is not available; all outcomes stated in methods were } \\
\text { reported in results. }\end{array}$ \\
\hline Other bias & Low risk & None obvious. \\
\hline
\end{tabular}




Allocation: parallel, randomised
Blind: single-blind to assessor
Setting: hospital (inpatient and outpatient), multicentre
Country: Thailand
Length of study: 26 months

\section{Participants}

Diagnosis: treatment-resistant schizophrenia (DSM-IV)

\section{Total $\mathbf{N}$ at randomisation $=51$}

The following characteristics are based on completers $(n=45)$.

Sex: male 21 , female 24

Age (years): mean 32.7, SD 8.4

Length of illness (years): mean 11.9, SD 6.8

History: with acute psychotic exacerbation; no serious medical condition, as assessed by history, physical examination, and pertinent laboratory tests

Exclusion criteria: known hypersensitivity to the medications used in modified ECT (thiopental sodium and succinylcholine).

Content: the ECT devices were a MECTA SR1 and Thymatron DGx. The traditional bilateral electrode placement was used throughout. Flexibility was allowed to schedule each treatment within a 3-day window. Participants who did not receive treatment within this window were considered early leavers

Frequency: once a week for 1 month ( 4 treatments), then twice a week for 5 months (10 treatments)

Course of treatment (ECT): 14 sessions

Treatment duration: 6 months

Control group A $(n=15):$ ECT + flupenthixol

Content: ECT used same as above; the dosage schedule of flupenthixol was fixed after 8 weeks of beginning treatment: $12 \mathrm{mg} / \mathrm{d}$ during the first week and increased up to $24 \mathrm{mg} / \mathrm{d}$ depending on tolerability.

Frequency: same as above for ECT, not stated for flupenthixol

Treatment duration: 6 months

Control group B $(n=15)$ : Flupenthixol alone

Content: the usage and dosage of flupenthixol was same as above.

Frequency: not stated

Treatment duration: 6 months

\section{Outcomes}

Mental state: assessed by BPRS, MMSE

General functioning: assessed by GAF

Unable to use: relapse (number for compared groups not reported); results of the parametric regression analysis of the relapse time (not predefined in the protocol) 
Chanpattana 1999 (Continued)

Notes

Contact information: Department of Psychiatry, Srinakharinwirot University, Vajira Hospital, Samsen, Dusit, Bangkok 10300, Thailand

\section{Risk of bias}

\begin{tabular}{lll}
\hline Bias & Authors' judgement & Support for judgement \\
\hline $\begin{array}{l}\text { Random sequence genera- } \\
\text { tion (selection bias) }\end{array}$ & Unclear risk & Quote: "...were randomized to three treatment groups..." (p.182) \\
& & $\begin{array}{l}\text { Comments: The author described a random component in the sequence gen- } \\
\text { eration process, but no details were provided on randomisation method. }\end{array}$
\end{tabular}

\begin{tabular}{ll}
\hline $\begin{array}{l}\text { Allocation concealment } \\
\text { (selection bias) }\end{array}$ & Unclear risk
\end{tabular} $\begin{aligned} & \text { Comments: The author did not describe the allocation concealment. Insuffi- } \\
& \text { cient information to permit judgement of 'low risk' or 'high risk'. }\end{aligned}$

\begin{tabular}{|c|c|c|}
\hline $\begin{array}{l}\text { Blinding of participants } \\
\text { and personnel (perfor- }\end{array}$ & High risk & $\begin{array}{l}\text { Comments: The participants and personnel could not be blinded as } 1 \text { group } \\
\text { did not use ECT, and no sham-ECT was used. }\end{array}$ \\
\hline
\end{tabular}

$$
\text { mance bias) }
$$

All outcomes

\begin{tabular}{lll}
\hline $\begin{array}{l}\text { Blinding of outcome as- } \\
\begin{array}{l}\text { sessment (detection bias) } \\
\text { All outcomes }\end{array}\end{array}$ & Low risk & $\begin{array}{l}\text { Quote: "Five psychiatric nurses served as raters, and they were blind to the } \\
\text { treatment modality." (p.181) } \\
\text { Comments: The blinding of outcome assessment was ensured. }\end{array}$ \\
\hline $\begin{array}{l}\text { Incomplete outcome data } \\
\text { (attrition bias) }\end{array}$ & Unclear risk & $\begin{array}{l}\text { Comments: A total of } 6 \text { participants }(11.8 \%) \text { dropped out or withdrew consent } \\
\text { due to fear of ECT or denial of illness. Intention-to-treat analysis was used, but } \\
\text { the number of participants randomised to each group was not stated. Conse- } \\
\text { quently, only completer data could be used. }\end{array}$
\end{tabular}

\begin{tabular}{lll}
\hline $\begin{array}{l}\text { Selective reporting (re- } \\
\text { porting bias) }\end{array}$ & Low risk & $\begin{array}{l}\text { Comments: The protocol is not available; all outcomes stated in methods re- } \\
\text { ported in results. }\end{array}$ \\
\hline Other bias & Low risk & None obvious. \\
\hline
\end{tabular}

\section{Chen 2012}

Allocation: parallel, randomised
Blind: not stated
Setting: hospital (inpatient and outpatient), single centre
Country: China
Length of study: 12 weeks

\section{Participants}

Diagnosis: treatment-resistant schizophrenia (CCMD-3)

\section{Total $\mathbf{N}$ at randomisation $=71$}

Sex: male 39, female 32

Age (years): mean 31.88, SD 9.2

Length of illness (years): mean 16.1, SD 11.6

Inclusion criteria: failure after 3 types of antipsychotics (at least 2 types of antipsychotics with different chemical structure; equivalent dose of chlorpromazine more than $600 \mathrm{mg} / \mathrm{d}$, each drug used more 
Chen 2012 (Continued)

than 6 weeks, no significant improvement or response) over the last 5 years; social adaptation dysfunction: PANSS score $>60$.

Exclusion criteria: people with severe physical or brain illness; alcohol or drug abusers.

ECT group ( $\mathrm{n}=36$ ): MECT + clozapine
Content: the mean dose of clozapine was $275 \pm 25 \mathrm{mg} / \mathrm{d}$ when combined with MECT. The dosage of
clozapine was $1 / 2$ to $2 / 3$ times that of 'clozapine alone' group. The ECT device was Thymatron. Partici-
pants did not receive benzodiazepines, antiepileptic drugs, haloperidol, or other treatment.
Frequency: for MECT, 3 times a week (for first 3 weeks), then twice a week for the other weeks; not stat-
ed for clozapine
Course of treatment (MECT): 12 sessions
Treatment duration: 12 weeks
Control group ( $=35):$ Clozapine alone
Content: the mean dose of clozapine was $355 \pm 65 \mathrm{mg} / \mathrm{d}$.
Frequency: not stated
Treatment duration: 12 weeks

\begin{tabular}{l} 
Response to treatment: clinically significant response to treatment ${ }^{\star}$ \\
Mental state: assessed by PANSS \\
Adverse events \\
\hline
\end{tabular}

Notes $\quad{ }^{*}$ Clinically significant response assessed by the reducing score rate of PANSS:

1) cure: reducing rate $\geq 75 \%$;

2) significant improvement: reducing rate between $50 \%$ and $75 \%$;

3) improvement: reducing rate between $25 \%$ and $49 \%$;

4) no clinical response: reducing rate $<25 \%$.

The clinically significant response to treatment defined as cure + significant improvement.

Contact information: Shihezi Oasis Hospital, Shihezi, Xinjiang, China

\section{Risk of bias}

\begin{tabular}{lll}
\hline Bias & Authors' judgement & Support for judgement \\
\hline $\begin{array}{l}\text { Random sequence genera- } \\
\text { tion (selection bias) }\end{array}$ & Unclear risk & $\begin{array}{l}\text { Quote: "...were divided randomly into two groups..." (p.58) } \\
\text { Comments: The author described a random component in the sequence gen- } \\
\text { eration process, but no details were provided on randomisation method. }\end{array}$ \\
\hline $\begin{array}{l}\text { Allocation concealment } \\
\text { (selection bias) }\end{array}$ & Unclear risk & $\begin{array}{l}\text { Comments: The author did not describe the allocation concealment. Insuffi- } \\
\text { cient information to permit judgement of 'low risk' or 'high risk'. }\end{array}$ \\
$\begin{array}{l}\text { Blinding of participants } \\
\text { and personnel (perfor- } \\
\text { mance bias) } \\
\text { All outcomes }\end{array}$ & High risk & $\begin{array}{l}\text { Comments: The participants and personnel could not be blinded as } 1 \text { group } \\
\text { did not use ECT, and no sham-ECT was used. }\end{array}$ \\
\hline
\end{tabular}


Chen 2012 (Continued)

Blinding of outcome as- Unclear risk Comments: The author did not describe the blinding of outcome assessment. sessment (detection bias) Insufficient information to permit judgement of 'low risk' or 'high risk'.

All outcomes

Incomplete outcome data Low risk Comments: No missing outcome data.
(attrition bias)

(attrition bias)

All outcomes

$\begin{array}{ll}\begin{array}{l}\text { Selective reporting (re- } \\ \text { porting bias) }\end{array} & \text { Low risk } \\ \end{array}$

Other bias Low risk None obvious.

Goswami 2003

$\begin{array}{ll}\text { Methods } & \text { Allocation: parallel, randomised } \\ \text { Blind: double-blind } \\ \text { Setting: hospital (inpatient), single centre } \\ \text { Country: India } \\ \text { Length of study: } 4 \text { weeks }\end{array}$

Participants

Diagnosis: treatment-resistant schizophrenia (DSM-IV)

Total $\mathbf{N}$ at randomisation $=31$

The following characteristics are based on completers $(n=25)$.

Sex: male 16 , female 9

Age (years): mean 29.8, SD 8.54

Length of illness (years): mean 7.6, SD not stated

History: all patients were screened for persistent illness of 5 or more years' duration with no satisfactory social and occupational functioning possible; it was also verified through prescriptions and hospital records that each patient had at least 3 periods of treatment in the preceding 5 years with neuroleptics of at least 2 different chemical classes, at doses equivalent to or greater than $1000 \mathrm{mg} / \mathrm{d}$ of chlorpromazine equivalent for a period of 6 weeks each without significant relief.

Exclusion criteria: BPRS score $<45$; CGI-S $\leq 4$.

Content: bitemporal leads were applied and the parameters on the ECT machine (MECTA-SR1 apparatus) were selected to deliver a brief pulse intensity of $50 \%$ to $200 \%$ of seizure threshold. Anaesthesia was induced in all participants with injection thiopental sodium 4 to $5 \mathrm{mg} / \mathrm{kg}$. ECT group were administered injection succinylcholine $0.6 \mathrm{mg} / \mathrm{kg}$ and were manually ventilated with $100 \%$ oxygen using face mask with Magill's circuit connected to Boyle's anaesthesia apparatus. To ensure seizure duration of 25 seconds, the seizures were recorded by 2 electrodes placed bilaterally with unipolar frontal electroencephalogram lead.

All participants were given chlorpromazine up to $1000 \mathrm{mg} / \mathrm{d}$. The dose was titrated based on clinical response and side effects.

Frequency: 3 times a week 
Treatment duration: 2 to 4 weeks

Control group $(n=15)$ : Sham-ECT + chlorpromazine

Content: anaesthesia was induced in all participants with injection thiopental sodium 4 to $5 \mathrm{mg} / \mathrm{kg}$. In sham-ECT group, ECT was not given, however after induction of anaesthesia manual ventilation was done till the participant returned to adequate spontaneous respiration.

All participants were given chlorpromazine up to $1000 \mathrm{mg} / \mathrm{d}$. The dose was titrated based on clinical response and side effects.

Frequency: same as above

Treatment duration: same as above

Extrapyramidal side effects, if any, were managed by the trihexyphenidyl up to $6 \mathrm{mg} / \mathrm{d}$. In uncontrolled agitated psychotic participants, intravenous diazepam and promethazine were also used as and when required.

\begin{tabular}{ll}
\hline Outcomes & Mental state: assessed by BPRS \\
& Service use: number readmitted \\
& Unable to use: \\
& Response to treatment ${ }^{*}$ \\
& Rescue medication, total daily antipsychotics received, global state assessed by CGI-S (not predefined \\
in the protocol) & Satisfaction (data not reported) \\
Notes & *Improvement was defined as a $20 \%$ decrease in BPRS total score plus either post-treatment CGI rating \\
& mildly ill (<3) or a post-treatment score of 35 or less on BPRS.
\end{tabular}

Contact information: Lady Hardinge Medical College \& Associated Hospitals, New Delhi 110001, India

\section{Risk of bias}

Bias Authors' judgement Support for judgement

Random sequence genera- Low risk Quote: "Using a random number table, the patients were randomly allocated tion (selection bias) to treatment groups..." (p.27)

Comments: The author described a random component in the sequence generation process.

Allocation concealment Low risk
(selection bias)

Quote: "The patients were randomly allocated to treatment groups by anaesthesia team and none of the psychiatrists knew the blind." (p.27)

Comments: Allocation concealment was ensured.

\begin{tabular}{|c|c|c|}
\hline $\begin{array}{l}\text { Blinding of participants } \\
\text { and personnel (perfor- } \\
\text { mance bias) } \\
\text { All outcomes }\end{array}$ & Low risk & $\begin{array}{l}\text { Quote: "The patients were randomly allocated to treatment groups } \\
\text { by anaesthesia team and none of the psychiatrists knew the blind." (p.27) "... } \\
\text { the study had a design of random allocation of patients to treatment groups } \\
\text { and is conducted double blind..." (p.28) }\end{array}$ \\
\hline & & Comments: Blinding of participants and personnel was ensured. \\
\hline
\end{tabular}

\begin{tabular}{|c|c|c|}
\hline $\begin{array}{l}\text { Blinding of outcome as- } \\
\text { sessment (detection bias) }\end{array}$ & Unclear risk & $\begin{array}{l}\text { Comments: The author did not describe the blinding of outcome assessment. } \\
\text { Insufficient information to permit judgement of 'low risk' or 'high risk'. }\end{array}$ \\
\hline
\end{tabular}

All outcomes 
Goswami 2003 (Continued)

Incomplete outcome data Unclear risk (attrition bias)

All outcomes
Quote: "... 31 gave written informed consent. Five patients did not complete the study; one more patient was excluded as her diagnosis changed to schizoaffective disorder." (p.27)

Comments: The attrition rate is $19.4 \%$, the author did not describe the reason of attrition and did not perform ITT analysis.

\section{Selective reporting (re- High risk} porting bias)
Comments: The protocol is not available, however some outcomes in this review were not reported (response to treatment, satisfaction).

Other bias Low risk None obvious.

\section{Jiang 2009}

Methods

Blind: not stated

Setting: hospital (inpatient), single centre

Country: China

Length of study: 12 weeks

\section{Participants}

Diagnosis: treatment-resistant schizophrenia (CCMD-3)

\section{Total $\mathbf{N}$ at randomisation $=69$}

The following characteristics are based on completers $(n=67)$.

Sex: male 47 , female 20

Age (years): mean 38.3, SD 7.2

Length of illness (months): mean 151.3, SD 61.2

History: failure after 3 types of antipsychotics (at least 2 types of antipsychotics with different chemical structures, each drug used more than 6 weeks, no significant improvement in mental symptoms) over the last 5 years.

Exclusion criteria: people with severe physical illness; alcohol or drug abusers.

\section{Interventions}

ECT group $(n=34)$ : MECT + risperidone

Content: the ECT device was Thymatron; the dosage of risperidone was 4 to $8 \mathrm{mg} / \mathrm{d}$.

Frequency: for MECT, twice or 3 times a week (once daily in the next weeks of treatment depending on the situation); not stated for risperidone

Course of treatment (MECT): 8 to 12 sessions

Treatment duration: 12 weeks

Control group $(n=35)$ : Risperidone alone

Content: the usage and dosage of risperidone was same as above.

Frequency: not stated

Treatment duration: 12 weeks 
Jiang 2009 (Continued)

Outcomes
Response to treatment: clinically significant response to treatment*

Cognitive functioning: assessed by WCST

Acceptability of treatment: leaving the study early

Mental state: assessed by PANSS

General functioning: assessed by GAF

Adverse events: assessed by TESS

1) cure: reducing rate $\geq 80 \%$;

2) significant improvement: reducing rate between $50 \%$ and $79 \%$;

3) improvement: reducing rate between $30 \%$ and $49 \%$;

4) no clinical response: reducing rate $<30 \%$.

The clinically significant response to treatment was defined as cure + significant improvement.

Contact information: jxq0481@163.com; Department of Psychiatry, Third Hospital of Quzhou, Zhejiang, China

\section{No reply.}

\section{Risk of bias}

\begin{tabular}{lll}
\hline Bias & Authors' judgement & Support for judgement \\
\hline $\begin{array}{ll}\text { Random sequence genera- } \\
\text { tion (selection bias) }\end{array}$ & Low risk & $\begin{array}{l}\text { Quote: "...were divided randomly into two groups using random number ta- } \\
\text { ble..." (p.80) }\end{array}$ \\
& $\begin{array}{l}\text { Comments: The author described a random component in the sequence gen- } \\
\text { eration process. }\end{array}$
\end{tabular}

\begin{tabular}{ll}
\hline $\begin{array}{l}\text { Allocation concealment } \\
\text { (selection bias) }\end{array}$ & Unclear risk
\end{tabular} $\begin{aligned} & \text { Comments: The author did not describe the allocation concealment. Insuffi- } \\
& \text { cient information to permit judgement of 'low risk' or 'high risk'. }\end{aligned}$

\begin{tabular}{|c|c|c|}
\hline $\begin{array}{l}\text { Blinding of participants } \\
\text { and personnel (perfor- }\end{array}$ & High risk & $\begin{array}{l}\text { Comments: The participants and personnel could not be blinded as } 1 \text { group } \\
\text { did not use ECT, and no sham-ECT was used. }\end{array}$ \\
\hline
\end{tabular}
mance bias)

All outcomes

\begin{tabular}{lll}
\hline $\begin{array}{l}\text { Blinding of outcome as- } \\
\text { sessment (detection bias) }\end{array}$ & Unclear risk & $\begin{array}{l}\text { Comments: The author did not describe the blinding of outcome assessment. } \\
\text { Insufficient information to permit judgement of 'low risk' or 'high risk'. }\end{array}$
\end{tabular}

\begin{tabular}{lll}
\hline $\begin{array}{l}\text { Incomplete outcome data } \\
\text { (attrition bias) } \\
\text { All outcomes }\end{array}$ & Low risk & $\begin{array}{l}\text { Comments: A total 2 participants (2.9\%) dropped out from the MECT + risperi- } \\
\text { done group due to adverse events (stress ulcer bleeding, headache, nausea } \\
\text { and vomiting). The attrition bias is small, may not impact the results. }\end{array}$ \\
\hline $\begin{array}{l}\text { Selective reporting (re- } \\
\text { porting bias) }\end{array}$ & Low risk & $\begin{array}{l}\text { Comments: The protocol is not available; all outcomes stated in methods re- } \\
\text { ported in results. }\end{array}$ \\
\hline Other bias & Low risk & None obvious. \\
\hline
\end{tabular}




Allocation: parallel, randomised
Blind: not stated
Setting: hospital (inpatient), single centre
Country: China
Length of study: 8 weeks

Participants

Diagnosis: treatment-resistant schizophrenia (CCMD-2-R/DSM-IV)

Total $\mathbf{N}$ at randomisation $=162$

Sex: male 102, female 60

Age (years): range 21 to 74

Length of illness (years): mean 7.9; range 3 to 12

History: patients received at least 3 types of antipsychotics.

Exclusion criteria: patients with liver and kidney disease, brain organic disease, or other ECT contraindications.

Interventions $\quad$ ECT group $(n=81)$ : MECT + ziprasidone

Content: the initial dose of ziprasidone was $20 \mathrm{mg} / \mathrm{d}$, increased to $80 \mathrm{mg} / \mathrm{d}$ according to participants' tolerance. Participants also received MECT therapy.

Frequency: for MECT, 3 times a week for the first 2 weeks, then once a week for the next 6 weeks; not stated for ziprasidone.

Course of treatment (MECT): 12 sessions

Treatment duration: 8 weeks

Control group $(n=81)$ : Clozapine + ziprasidone

Content: the usage and dosage of ziprasidone was same as above. The initial dose of clozapine was 50 $\mathrm{mg} / \mathrm{d}$, increased to $400 \mathrm{mg} / \mathrm{d}$ within a week.

Frequency: twice daily for clozapine; not stated for ziprasidone.

Treatment duration: 8 weeks

Outcomes Response to treatment: clinically significant response to treatment ${ }^{\star}$

Mental state: assessed by BPRS

Adverse events: assessed by TESS

Notes

${ }^{\star}$ Clinically significant response assessed by the reducing score rate of BPRS:

1) cure: reducing rate $\geq 75 \%$;

2) significant improvement: reducing rate between $50 \%$ and $74 \%$;

3) improvement: reducing rate between $25 \%$ and $49 \%$;

4) no clinical response: reducing rate $<25 \%$.

The clinically significant response to treatment was defined as cure + significant improvement. 
Contact information: Wards 2 of Xuzhou Mental Hospital, Xuzhou, Jiangsu, China

\section{Risk of bias}

\begin{tabular}{lll}
\hline Bias & Authors' judgement & Support for judgement \\
\hline $\begin{array}{ll}\text { Random sequence genera- } \\
\text { tion (selection bias) }\end{array}$ & Unclear risk & Quote: "...were divided randomly into two groups..." (p.1394) \\
& & $\begin{array}{l}\text { Comments: The author described a random component in the sequence gen- } \\
\text { eration process, but no details were provided on randomisation method. }\end{array}$
\end{tabular}

\begin{tabular}{|c|c|c|}
\hline $\begin{array}{l}\text { Allocation concealment } \\
\text { (selection bias) }\end{array}$ & Unclear risk & $\begin{array}{l}\text { Comments: The author did not describe the allocation concealment. Insuffi- } \\
\text { cient information to permit judgement of 'low risk' or 'high risk'. }\end{array}$ \\
\hline $\begin{array}{l}\text { Blinding of participants } \\
\text { and personnel (perfor- } \\
\text { mance bias) } \\
\text { All outcomes }\end{array}$ & High risk & $\begin{array}{l}\text { Comments: The participants and personnel could not be blinded as } 1 \text { group } \\
\text { did not use ECT, and no sham-ECT was used. }\end{array}$ \\
\hline $\begin{array}{l}\text { Blinding of outcome as- } \\
\text { sessment (detection bias) } \\
\text { All outcomes }\end{array}$ & Unclear risk & $\begin{array}{l}\text { Comments: The author did not describe the blinding of outcome assessment. } \\
\text { Insufficient information to permit judgement of 'low risk' or 'high risk'. }\end{array}$ \\
\hline $\begin{array}{l}\text { Incomplete outcome data } \\
\text { (attrition bias) } \\
\text { All outcomes }\end{array}$ & Low risk & Comments: No missing outcome data. \\
\hline $\begin{array}{l}\text { Selective reporting (re- } \\
\text { porting bias) }\end{array}$ & Low risk & $\begin{array}{l}\text { Comments: The protocol is not available; all outcomes stated in methods re- } \\
\text { ported in results. }\end{array}$ \\
\hline Other bias & Low risk & None obvious. \\
\hline
\end{tabular}

Lin 2014

\begin{tabular}{l} 
Allocation: parallel, randomised \\
Blind: not stated \\
Setting: hospital (inpatient), single centre \\
Country: China \\
Length of study: 8 weeks \\
\hline
\end{tabular}

Participants

Diagnosis: treatment-resistant schizophrenia (ICD-10)

Total $\mathbf{N}$ at randomisation $=78$

Sex: male 49, female 29

Age (years): mean 38.25, SD 1.48

Length of illness (years): mean 6.92, SD 1.38

History: poor social function; without history of drug abuse.

Exclusion criteria: patients who are allergic to treatment medications; pregnant women and lactating women; patients with severe chronic illnesses, hypotension, or epilepsy; patients with heart, liver, or 
Lin 2014 (Continued)

kidney dysfunction; patients who do not co-operate with the treatment; patients with incomplete clinical data.

ECT group ( $\mathrm{n}=39): \mathrm{MECT}+$ ziprasidone
Content: the initial dose of ziprasidone was $20 \mathrm{mg} / \mathrm{d}$, increased to 80
participant's condition. Participants also received MECT therapy.
Frequency: not stated
Course of treatment (MECT): not stated
Treatment duration: 8 weeks
Control group ( $\mathrm{n}=39$ ): ziprasidone alone
Content: the usage and dosage of ziprasidone was same as above.
Frequency: not stated
Treatment duration: 8 weeks

Outcomes Mental state: assessed by BPRS

Adverse events

Notes Contact information: Second Department, Fourth People's Hospital of Urumqi, China

\section{Risk of bias}

\begin{tabular}{|c|c|c|}
\hline Bias & Authors' judgement & Support for judgement \\
\hline \multirow{2}{*}{$\begin{array}{l}\text { Random sequence genera- } \\
\text { tion (selection bias) }\end{array}$} & Unclear risk & Quote: "...were divided randomly into two groups..." (p.202) \\
\hline & & $\begin{array}{l}\text { Comments: The author described a random component in the sequence gen- } \\
\text { eration process, but no details were provided on randomisation method. }\end{array}$ \\
\hline $\begin{array}{l}\text { Allocation concealment } \\
\text { (selection bias) }\end{array}$ & Unclear risk & $\begin{array}{l}\text { Comments: The author did not describe the allocation concealment. Insuffi- } \\
\text { cient information to permit judgement of 'low risk' or 'high risk'. }\end{array}$ \\
\hline $\begin{array}{l}\text { Blinding of participants } \\
\text { and personnel (perfor- } \\
\text { mance bias) } \\
\text { All outcomes }\end{array}$ & High risk & $\begin{array}{l}\text { Comments: The participants and personnel could not be blinded as } 1 \text { group } \\
\text { did not use ECT, and no sham-ECT was used. }\end{array}$ \\
\hline $\begin{array}{l}\text { Blinding of outcome as- } \\
\text { sessment (detection bias) } \\
\text { All outcomes }\end{array}$ & Unclear risk & $\begin{array}{l}\text { Comments: The author did not describe the blinding of outcome assessment. } \\
\text { Insufficient information to permit judgement of 'low risk' or 'high risk'. }\end{array}$ \\
\hline $\begin{array}{l}\text { Incomplete outcome data } \\
\text { (attrition bias) } \\
\text { All outcomes }\end{array}$ & Low risk & Comments: No missing outcome data. \\
\hline $\begin{array}{l}\text { Selective reporting (re- } \\
\text { porting bias) }\end{array}$ & Low risk & $\begin{array}{l}\text { Comments: The protocol is not available; all outcomes stated in methods re- } \\
\text { ported in results. }\end{array}$ \\
\hline Other bias & Low risk & None obvious. \\
\hline
\end{tabular}


Liu 2010

\begin{tabular}{ll}
\hline Methods & Allocation: parallel, randomised \\
Blind: not stated \\
Setting: hospital (inpatient), single centre \\
Country: China \\
Length of study: 4 weeks
\end{tabular}

\begin{tabular}{ll}
\hline Participants & Diagnosis: schizophrenia (CCMD-3) \\
Total $\mathbf{N}$ at randomisation $=72$ \\
Sex: male 35, female 37 \\
Age (years): mean 38.42, SD 6.15 \\
Length of illness (years): not stated \\
History: not stated \\
Exclusion criteria: patients with severe physical illness; alcohol or drug abusers.
\end{tabular}

ECT group $(n=35):$ MECT + antipsychotics
Content: participants received antipsychotic therapy such as chlorpromazine, clozapine, per-
phenazine, and risperidone, and also received MECT. The ECT device was Thymatron.
Frequency: 3 times a week for MECT; not stated for antipsychotics
Course of treatment (MECT): 12 sessions
Treatment duration: 28 days

Control group ( $n=37)$ : Antipsychotics alone

Content: participants received antipsychotic therapy such as chlorpromazine, clozapine, perphenazine, and risperidone.

Frequency: not stated

Treatment duration: 28 days

\begin{tabular}{|c|c|c|}
\hline \multirow[t]{3}{*}{ Outcomes } & \multicolumn{2}{|c|}{ Mental state: assessed by SAPS, SANS } \\
\hline & \multicolumn{2}{|l|}{ Unable to use: } \\
\hline & \multicolumn{2}{|c|}{ Response to treatment (data not reported) } \\
\hline Notes & \multicolumn{2}{|c|}{ Contact information: Shaoyang Psychiatric Hospital, Shaoyang, Hunan, China } \\
\hline \multicolumn{3}{|l|}{ Risk of bias } \\
\hline Bias & Authors' judgement & Support for judgement \\
\hline \multirow{2}{*}{$\begin{array}{l}\text { Random sequence genera- } \\
\text { tion (selection bias) }\end{array}$} & Unclear risk & Quote: "...were divided randomly into two groups..." (p.29) \\
\hline & & $\begin{array}{l}\text { Comments: The author described a random component in the sequence gen- } \\
\text { eration process, but no details were provided on randomisation method. }\end{array}$ \\
\hline $\begin{array}{l}\text { Allocation concealment } \\
\text { (selection bias) }\end{array}$ & Unclear risk & $\begin{array}{l}\text { Comments: The author did not describe the allocation concealment. Insuffi- } \\
\text { cient information to permit judgement of 'low risk' or 'high risk'. }\end{array}$ \\
\hline
\end{tabular}


Liu 2010 (Continued)

Blinding of participants High risk Comments: The participants and personnel could not be blinded as 1 group and personnel (perfordid not use ECT, and no sham-ECT was used. mance bias)

All outcomes

\begin{tabular}{|c|c|c|}
\hline $\begin{array}{l}\text { Blinding of outcome as- } \\
\text { sessment (detection bias) }\end{array}$ & Unclear risk & $\begin{array}{l}\text { Comments: The author did not describe the blinding of outcome assessment. } \\
\text { Insufficient information to permit judgement of 'low risk' or 'high risk'. }\end{array}$ \\
\hline
\end{tabular}

Incomplete outcome data Low risk Comments: No missing outcome data.
(attrition bias)

(attrition bias)

All outcomes

\begin{tabular}{lll}
\hline $\begin{array}{l}\text { Selective reporting (re- } \\
\text { porting bias) }\end{array}$ & High risk & $\begin{array}{l}\text { Comments: The protocol is not available; all outcomes stated in methods oth- } \\
\text { er than overall efficiency rate reported in results. }\end{array}$ \\
\hline Other bias & Low risk & None obvious. \\
\hline
\end{tabular}

Petrides 2015

\begin{tabular}{l} 
Allocation: parallel, randomised \\
Blind: single-blind to assessor \\
Setting: hospital (inpatient), multicentre \\
Country: USA \\
Length of study: 8 weeks \\
\hline
\end{tabular}

Participants

Diagnosis: treatment-resistant schizophrenia (DSM-IV)

Total $\mathbf{N}$ at randomisation $=39$

Sex: male 28 , female 11

Age (years) ${ }^{\star}$ : mean 35.7, SD 2.27 for ECT-treated group; mean 42.78, SD 1.82 for non-ECT group

Length of illness (years): range $>2$

History: resistance to at least 2 antipsychotics; clozapine resistance; a baseline BPRS score of at least moderate (score of 4) on 1 of the 4 psychotic items (hallucinatory behaviour, suspiciousness, conceptual disorganisation, and unusual thought content) of the psychotic symptom sub scale or a score of 12 on these 4 items combined; a CGI-S rating of at least moderate (score of 4).

Exclusion criteria: schizoaffective disorder; bipolar disorder; current affective episode; ECT within 6 months; history of epilepsy; severe neurological or systemic disorder that could significantly affect cognition, behaviour, or mental status (other than tardive dyskinesia or neuroleptic-induced parkinsonism); psychoactive substance dependence (other than nicotine or caffeine) within 1 month prior to entering the study; a score > 18 on the 24-item HAM-D; clinical determination that mood stabilisers that could not be discontinued were necessary; pregnancy; affective disorders and prominent depressive symptoms.

Interventions

$$
\text { ECT group }(n=20): \text { ECT + clozapine }
$$

Content: participants remained on the clozapine dose at which they were on when entering the study. ECT was performed with bilateral placement using the Thymatron-DGx device (Somatics, Lake Bluff, IL). Seizure threshold was determined at the first treatment. Dosing at subsequent treatments was given at $50 \%$ above threshold. 
Petrides 2015 (Continued)

Frequency: for ECT, 3 times a week for the first 4 weeks, then twice a week for the next 4 weeks; not stated for clozapine

Course of treatment (ECT): 20 sessions

Treatment duration: 8 weeks

Control group ( $n=19)$ : Clozapine alone

Content: the usage and dosage of clozapine was same as above.

Frequency: not stated

Treatment duration: 8 weeks

Concurrent use of other antipsychotic medications and antidepressants was allowed as long as they were taken at a stable dose for at least 12 weeks before entering the study. Lorazepam, up to $6 \mathrm{mg} / \mathrm{d}$, or diphenhydramine, up to $100 \mathrm{mg}$, was used as needed for anxiety, agitation, or insomnia.

Response to treatment ${ }^{\star \star}$
Acceptability of treatment: leaving the study early
Mental state: assessed by BPRS ${ }^{\star \star \star}$, MMSE
Adverse events
Unable to use (not predefined in the protocol):
Global state assessed by CGI-S

Notes

${ }^{\star}$ The ECT-treated group was significantly younger than the clozapine group.

** The response criterion was defined as improvement $\geq 40 \%$ based on the psychotic symptom sub scale (PSYRATS), a CGI-severity rating of mild or less $(<3)$, and a CGI-I rating of much improved $(\leq 2)$.

***The data for BPRS were extracted from Figure 2 (p.56) using GetDate software; we performed a sensitivity analysis for these data.

Contact information: Petrides@lij.edu; Division of Psychiatry Research, The Zucker Hillside Hospital, North Shore-LIJ Health System

No reply.

\section{Risk of bias}

\begin{tabular}{lll}
\hline Bias & Authors' judgement & Support for judgement \\
\hline $\begin{array}{l}\text { Random sequence genera- } \\
\text { tion (selection bias) }\end{array}$ & Unclear risk & Quote: "In an 8-week random-assignment study ..." (p.53) \\
& $\begin{array}{l}\text { Comments: The author described a random component in the sequence gen- } \\
\text { eration process, but no details were provided on randomisation method. }\end{array}$ \\
$\begin{array}{l}\text { Allocation concealment } \\
\text { (selection bias) }\end{array}$ & Unclear risk & $\begin{array}{l}\text { Comments: The author did not describe the allocation concealment. Insuffi- } \\
\text { cient information to permit judgement of 'low risk' or 'high risk'. }\end{array}$ \\
\hline $\begin{array}{l}\text { Blinding of participants } \\
\text { and personnel (perfor- } \\
\text { mance bias) }\end{array}$ & High risk & $\begin{array}{l}\text { Quote: "...incorporating non-blinded treatment and blinded assess- } \\
\text { ments..." (p.53) }\end{array}$ \\
\hline $\begin{array}{l}\text { Bll outcomes } \\
\text { sessment (detection bias) }\end{array}$ & Low risk & $\begin{array}{l}\text { Quote: "...incorporating non-blinded treatment and blinded assess- } \\
\text { ments..." (p.53) }\end{array}$ \\
\hline
\end{tabular}


Petrides 2015 (Continued)

All outcomes
Comments: The blinding of outcome assessment was ensured.
Incomplete outcome data Unclear risk (attrition bias)

All outcomes
Quote: "Of the 20 participants assigned to ECT plus clozapine, ... two (10\%) dropped out early and refused further ECT treatments, and one (5\%) was removed from the study because of persistence of involuntary movements. In the clozapine group, ... and three patients (15.8\%) dropped out because they refused to continue to participate in the ratings assessment while there was no change in their treatment." (p.54)

Comments: the attrition rates were more than $10 \%$ in two groups although balanced, and the reasons were various between the groups.

\begin{tabular}{lll}
\hline $\begin{array}{l}\text { Selective reporting (re- } \\
\text { porting bias) }\end{array}$ & Low risk & $\begin{array}{l}\text { Comments: The protocol is not available; all outcomes stated in methods re- } \\
\text { ported in results. }\end{array}$ \\
\hline Other bias & Low risk & $\begin{array}{l}\text { Funding: Supported by an RO1 grant from National Institute of Mental Health } \\
\text { to Dr Petrides (MH-603930). }\end{array}$ \\
\hline
\end{tabular}

Wang 2008

\begin{tabular}{ll} 
Methods & Allocation: parallel, randomised \\
Blind: not stated \\
Setting: hospital (inpatient), single centre \\
Country: China \\
Length of study: 12 weeks \\
\hline
\end{tabular}

Participants

Diagnosis: treatment-resistant schizophrenia (CCMD-3)

Total $\mathbf{N}$ at randomisation $=76$

Sex: male 46 , female 30

Age (years): mean 45.8, SD 12.2

Length of illness (years): range $>5$

History: PANSS score > 60; received at least 3 types of antipsychotics, but never reached the clinically significant standard (reducing score rate of PANSS $<50 \%$ ).

Exclusion criteria: patients with severe physical illness; alcohol or drug abusers.

Interventions $\quad$ ECT group $(n=38)$ : MECT + antipsychotics (clozapine and risperidone)

Content: after 1-week wash-out period, clozapine and risperidone were used, and the treatment dosage was reached within 1 to 2 weeks. The mean dosage of clozapine was $260 \pm 86 \mathrm{mg} / \mathrm{d}$, and the mean dosage of risperidone was $3.3 \pm 0.8 \mathrm{mg} / \mathrm{d}$. ECT was administered in the third week of treatment; the device was Thymatron.

Frequency: once every other day for ECT; not stated for antipsychotics

Course of treatment (MECT): 6 sessions

Treatment duration: 12 weeks

Control group ( $n=38)$ : Antipsychotics (clozapine and risperidone) 
Wang 2008 (Continued)

Content: the usage schedule was same as above. The mean dosage of clozapine was $300 \pm 98 \mathrm{mg} / \mathrm{d}$, and the mean dosage of risperidone was $3.0 \pm 0.6 \mathrm{mg} / \mathrm{d}$.

Frequency: not stated

Treatment duration: 12 weeks

\begin{tabular}{ll}
\hline Outcomes & Response to treatment: clinically significant response to treatment ${ }^{\star}$ \\
Mental state: assessed by PANSS \\
Unable to use: \\
Memory assessed by WMS (data in control group not reported); adverse events (data not reported for 2 \\
groups)
\end{tabular}

Notes

${ }^{\star}$ Clinically significant response assessed by the reducing score rate of PANSS:

1) cure: reducing rate $\geq 75 \%$;

2) significant improvement: reducing rate $\geq 50 \%$;

3) improvement: reducing rate $\geq 25 \%$;

4) no clinical response: reducing rate $<25 \%$.

The overall efficiency defined as cure + significant improvement.

Contact information: zdz200601@126.com; Second Affiliated Hospital of Xianning College, Hubei, China

No reply.

\section{Risk of bias}

\begin{tabular}{|c|c|c|}
\hline Bias & Authors' judgement & Support for judgement \\
\hline \multirow{2}{*}{$\begin{array}{l}\text { Random sequence genera- } \\
\text { tion (selection bias) }\end{array}$} & Unclear risk & Quote: "...were randomly assigned to two groups..." (p.415) \\
\hline & & $\begin{array}{l}\text { Comments: The author described a random component in the sequence gen- } \\
\text { eration process, but no details were provided on randomisation method. }\end{array}$ \\
\hline $\begin{array}{l}\text { Allocation concealment } \\
\text { (selection bias) }\end{array}$ & Unclear risk & $\begin{array}{l}\text { Comments: The author did not describe the allocation concealment. Insuffi- } \\
\text { cient information to permit judgement of 'low risk' or 'high risk'. }\end{array}$ \\
\hline $\begin{array}{l}\text { Blinding of participants } \\
\text { and personnel (perfor- } \\
\text { mance bias) } \\
\text { All outcomes }\end{array}$ & High risk & $\begin{array}{l}\text { Comments: The participants and personnel could not be blinded as } 1 \text { group } \\
\text { did not use ECT, and no sham-ECT was used. }\end{array}$ \\
\hline $\begin{array}{l}\text { Blinding of outcome as- } \\
\text { sessment (detection bias) } \\
\text { All outcomes }\end{array}$ & Unclear risk & $\begin{array}{l}\text { Comments: The author did not describe the blinding of outcome assessment. } \\
\text { Insufficient information to permit judgement of 'low risk' or 'high risk'. }\end{array}$ \\
\hline $\begin{array}{l}\text { Incomplete outcome data } \\
\text { (attrition bias) } \\
\text { All outcomes }\end{array}$ & Low risk & Comments: No missing outcome data. \\
\hline $\begin{array}{l}\text { Selective reporting (re- } \\
\text { porting bias) }\end{array}$ & Unclear risk & $\begin{array}{l}\text { Comments: The protocol is not available; all outcomes stated in methods re- } \\
\text { ported in results. However, data for memory in the control group were not re- } \\
\text { ported, and data for adverse events were not reported for } 2 \text { groups. }\end{array}$ \\
\hline
\end{tabular}


Wang 2008 (Continued)
Other bias
Low risk
None obvious.

Wang 2011

$\begin{array}{ll}\text { Methods } & \text { Allocation: parallel, randomised } \\ \text { Blind: not stated } & \text { Setting: hospital (inpatient), single centre } \\ \text { Country: China } \\ \text { Length of study: } 12 \text { weeks }\end{array}$

Participants

Diagnosis: treatment-resistant schizophrenia (CCMD-3)

Total $\mathbf{N}$ at randomisation $=74$

Sex: male 37 , female 37

Age (years): mean 26.5, SD 10.2

Length of illness (years): mean 15.7, SD 12.8

History: failure after more than 3 types of antipsychotics (at least 2 types of antipsychotics with different chemical structure; each drug with full dose used more than 6 weeks, no significant improvement or response) over the last 5 years; PANSS score $>60$.

Exclusion criteria: patients with severe physical illness or mental retardation; alcohol or drug abuser.

Interventions $\quad$ ECT group $(n=37)$ : MECT + clozapine

Content: keep the same dose of clozapine as previous (mean dose: $325 \pm 45 \mathrm{mg} / \mathrm{d}$ ). The ECT device was Thymatron.

Frequency: once daily for the first 3 days, then 2 to 3 times a week for ECT; not stated for clozapine

Course of treatment (MECT): 12 sessions

Treatment duration: 12 weeks

Control group $(n=37)$ : Clozapine alone

Content: the usage and dosage schedule of clozapine was same as above.

Frequency: not stated

Treatment duration: 12 weeks

\begin{tabular}{ll}
\hline Outcomes & Response to treatment: clinically significant response to treatment \\
Mental state: assessed by PANSS \\
Adverse events \\
Unable to use: \\
Memory assessed by WMS (data for control group not reported)
\end{tabular}

Notes

${ }^{*}$ Clinically significant response assessed by the reducing score rate of PANSS:

1) cure: reducing rate $\geq 75 \%$; 
Wang 2011 (Continued)
2) significant improvement: reducing rate between $50 \%$ and $74 \%$;
3) improvement: reducing rate between $25 \%$ and $49 \%$;
4) no clinical response: reducing rate $<25 \%$.

The clinically significant response to treatment was defined as cure + significant improvement.

Contact information: wangzhuawuhu@126.com; Fourth People's Hospital, Wuhu, Anhui, China

No reply.

\begin{tabular}{lll}
\hline Risk of bias & \\
\hline Bias & Authors' judgement & Support for judgement \\
\hline $\begin{array}{l}\text { Random sequence genera- } \\
\text { tion (selection bias) }\end{array}$ & Unclear risk & $\begin{array}{l}\text { Quote: "...were randomly assigned to receive..." (p.252) } \\
\text { Comments: The author described a random component in the sequence gen- } \\
\text { eration process, but no details were provided on randomisation method. }\end{array}$
\end{tabular}

\begin{tabular}{lll}
\hline $\begin{array}{l}\text { Allocation concealment } \\
\text { (selection bias) }\end{array}$ & Unclear risk & $\begin{array}{l}\text { Comments: The author did not describe the allocation concealment. Insuffi- } \\
\text { cient information to permit judgement of 'low risk' or 'high risk'. }\end{array}$ \\
\hline $\begin{array}{l}\text { Blinding of participants } \\
\text { and personnel (perfor- } \\
\begin{array}{l}\text { mance bias) } \\
\text { All outcomes }\end{array}\end{array}$ & High risk & $\begin{array}{l}\text { Comments: The participants and personnel could not be blinded as } 1 \text { group } \\
\text { did not use ECT, and no sham-ECT was used. }\end{array}$ \\
\end{tabular}

\begin{tabular}{|c|c|c|}
\hline $\begin{array}{l}\text { Blinding of outcome as- } \\
\text { sessment (detection bias) } \\
\text { All outcomes }\end{array}$ & Unclear risk & $\begin{array}{l}\text { Comments: The author did not describe the blinding of outcome assessment. } \\
\text { Insufficient information to permit judgement of 'low risk' or 'high risk'. }\end{array}$ \\
\hline
\end{tabular}

\begin{tabular}{lll}
\hline $\begin{array}{l}\text { Incomplete outcome data } \\
\text { (attrition bias) } \\
\text { All outcomes }\end{array}$ & Low risk & Comments: No missing outcome data. \\
\hline $\begin{array}{l}\text { Selective reporting (re- } \\
\text { porting bias) }\end{array}$ & Unclear risk & $\begin{array}{l}\text { Comments: The protocol is not available; all outcomes stated in methods re- } \\
\text { ported in results. However, data for memory in the control group were not re- } \\
\text { ported. }\end{array}$
\end{tabular}

\begin{tabular}{ll}
\hline Other bias $\quad$ Low risk $\quad$ None obvious. \\
\hline
\end{tabular}

\section{Wang 2013}

$\begin{array}{ll}\text { Methods } & \text { Allocation: parallel, randomised } \\ \text { Blind: not stated } \\ \text { Setting: hospital (inpatient), single centre } \\ \text { Country: China } \\ \text { Length of study: } 12 \text { weeks }\end{array}$

Participants

Diagnosis: treatment-resistant schizophrenia (CCMD-3)

Total $\mathbf{N}$ at randomisation $=72$ 
Wang 2013 (Continued)

Sex: male 43, female 29

Age (years): mean 45.5, SD 13.2

Length of illness (years): mean 13.2, SD 5.2

History: failure after 3 types of antipsychotics (at least 2 types of antipsychotics with different chemical structure, each drug used more than 6 weeks, no significant improvement in mental symptoms) over the last 5 years.

Exclusion criteria: not stated

ECT group ( $\mathrm{n}=36)$ : MECT + olanzapine
Content: the initial dose of olanzapine was low dose, increased to therapeu
$\begin{aligned} & 30 \mathrm{mg} / \mathrm{d} \text { ) within } 2 \text { weeks. The mean dose of olanzapine was } 5 \text { to } 10 \mathrm{mg} / \mathrm{d} \text {. } \mathrm{N} \\ & \text { used in the treatment. The ECT device was Thymatron. The traditional bilater } \\ & \text { was used throughout. The initial electrical dose was } 15 \% \text { energy percentag }\end{aligned}$
Frequency: 3 times a week for ECT; not stated for olanzapine
Course of treatment (MECT): 10 to 12 sessions
Treatment duration: 3 to 4 weeks
Control group ( $=36):$ Olanzapine alone
Content: the usage and dosage schedule of olanzapine was same as above.
Frequency: not stated
Treatment duration: same as above

Response to treatment: clinically significant response to treatment*
Cognitive functioning: memory deterioration
Adverse events

Notes

${ }^{\star}$ Clinically significant response assessed by the reducing score rate of PANSS:

1) cure: reducing rate $\geq 75 \%$;

2) significant improvement: reducing rate between $50 \%$ and $74 \%$;

3) improvement: reducing rate between $25 \%$ and $49 \%$;

4) no clinical response: reducing rate $<25 \%$.

The overall efficiency was defined as cure + significant improvement.

Remark: the author did not describe attrition, but the total number for whom response was assessed was not consistent with the number at randomisation. Hence, there was 1 additional participant in the 'MECT + olanzapine' group. We performed sensitivity analysis on the outcome response by removing this study.

Contact information: The Third People's Hospital, Ganzhou, Jiang Xi, China

\section{Risk of bias}

\begin{tabular}{lll}
\hline Bias & Authors' judgement & Support for judgement \\
\hline $\begin{array}{l}\text { Random sequence genera- } \\
\text { tion (selection bias) }\end{array}$ & Unclear risk & Quote: "...were divided randomly into two groups..." (p.99) \\
\hline
\end{tabular}


Comments: The author described a random component in the sequence generation process, but no details were provided on randomisation method.

\begin{tabular}{|c|c|c|}
\hline $\begin{array}{l}\text { Allocation concealment } \\
\text { (selection bias) }\end{array}$ & Unclear risk & $\begin{array}{l}\text { Comments: The author did not describe the allocation concealment. Insuffi- } \\
\text { cient information to permit judgement of 'low risk' or 'high risk'. }\end{array}$ \\
\hline $\begin{array}{l}\text { Blinding of participants } \\
\text { and personnel (perfor- } \\
\text { mance bias) } \\
\text { All outcomes }\end{array}$ & High risk & $\begin{array}{l}\text { Comments: The participants and personnel could not be blinded as } 1 \text { group } \\
\text { did not use ECT, and no sham-ECT was used. }\end{array}$ \\
\hline $\begin{array}{l}\text { Blinding of outcome as- } \\
\text { sessment (detection bias) } \\
\text { All outcomes }\end{array}$ & Unclear risk & $\begin{array}{l}\text { Comments: The author did not describe the blinding of outcome assessment. } \\
\text { Insufficient information to permit judgement of 'low risk' or 'high risk'. }\end{array}$ \\
\hline $\begin{array}{l}\text { Incomplete outcome data } \\
\text { (attrition bias) } \\
\text { All outcomes }\end{array}$ & Unclear risk & $\begin{array}{l}\text { Comments: The author did not describe attrition, but the total number for } \\
\text { whom response was assessed was not consistent with the number at randomi- } \\
\text { sation. Hence, there was } 1 \text { additional participant in the 'MECT + olanzapine' } \\
\text { group, and } 2 \text { participants fewer in the olanzapine-alone group. }\end{array}$ \\
\hline $\begin{array}{l}\text { Selective reporting (re- } \\
\text { porting bias) }\end{array}$ & Low risk & $\begin{array}{l}\text { Comments: The protocol is not available; all outcomes stated in methods re- } \\
\text { ported in results. }\end{array}$ \\
\hline Other bias & Low risk & None obvious. \\
\hline
\end{tabular}

Yang 2005

Allocation: parallel, randomised
Blind: not stated
Setting: hospital (inpatient), single centre
Country: China
Length of study: 8 weeks

Participants

Diagnosis: treatment-resistant schizophrenia (CCMD-3) with agitation and aggressive behaviour

Total $\mathbf{N}$ at randomisation $=60$

Sex: male 38 , female 22

Age (years): mean 18.62, SD 8.31

Length of illness (years): mean 6.25, SD 4.26

History: failure after 3 types of antipsychotics (at least 2 types of antipsychotics with different chemical structure) over the last 5 years; unrecovered social function; PANSS total score $>60$ (at least 3 of excitement items (P4, P7, G8, G14) score > 3; at least 2 of 3 attack risk items (S1, S2, S3) > 3).

Exclusion criteria: patients with physical diseases, organic diseases, or chronic decline schizophrenia; MECT or clozapine contraindications.

Interventions

$$
\text { ECT group }(n=30): \text { MECT + clozapine }
$$

Content: the initial dose of clozapine was $50 \mathrm{mg} / \mathrm{d}$, increased to 50 to $100 \mathrm{mg} / \mathrm{d}$ alternate days. The maximum dose of clozapine was no more than $300 \mathrm{mg} / \mathrm{d}$ when combining with MECT.

Frequency: 3 times a week for MECT, not stated for clozapine 
Yang 2005 (Continued)

Course of treatment (MECT): 6 to 12 sessions

Treatment duration: 8 weeks

Control group $(n=30)$ : Clozapine alone

Content: the usage and dose of clozapine was same as above. The maximum dose was $300 \mathrm{mg} / \mathrm{d}$ to 700 $\mathrm{mg} / \mathrm{d}$.

Frequency: not stated

Treatment duration: 8 weeks

$\begin{array}{ll}\text { Outcomes } & \text { Response to treatment: clinically significant response to treatment }{ }^{\star} \\ \text { Mental state: assessed by PANSS } \\ \text { Adverse events } \\ \text { Unable to use: } \\ \text { Adverse events assessed by TESS (only P value reported) }\end{array}$

Notes

${ }^{*}$ Clinically significant response assessed by the reducing score rate of PANSS:

1) cure: reducing rate $\geq 75 \%$;

2) significant improvement: reducing rate between $50 \%$ and $74 \%$;

3) improvement: reducing rate between $25 \%$ and $49 \%$;

4) no clinical response: reducing rate $<25 \%$.

The clinically significant response to treatment was defined as cure + significant improvement.

Contact information: Mental Health Institute, Shenzhen, Guangdong, China

\section{Risk of bias}

\begin{tabular}{lll}
\hline Bias & Authors' judgement & Support for judgement \\
\hline $\begin{array}{l}\text { Random sequence genera- } \\
\text { tion (selection bias) }\end{array}$ & Unclear risk & Quote: "...were divided randomly into two groups..." (p.485) \\
& $\begin{array}{l}\text { Comments: The author described a random component in the sequence gen- } \\
\text { eration process, but no details were provided on randomisation method. }\end{array}$
\end{tabular}

\begin{tabular}{lll}
\hline $\begin{array}{l}\text { Allocation concealment } \\
\text { (selection bias) }\end{array}$ & Unclear risk & $\begin{array}{l}\text { Comments: The author did not describe the allocation concealment. Insuffi- } \\
\text { cient information to permit judgement of 'low risk' or 'high risk'. }\end{array}$ \\
\hline $\begin{array}{l}\text { Blinding of participants } \\
\text { and personnel (perfor- } \\
\text { mance bias) }\end{array}$ & High risk & $\begin{array}{l}\text { Comments: The participants and personnel could not be blinded as } 1 \text { group } \\
\text { did not use ECT, and no sham-ECT was used. }\end{array}$ \\
\hline
\end{tabular}

\begin{tabular}{lll}
\hline $\begin{array}{l}\text { Blinding of outcome as- } \\
\text { sessment (detection bias) } \\
\text { All outcomes }\end{array}$ & Unclear risk & $\begin{array}{l}\text { Comments: The author did not describe the blinding of outcome assessment. } \\
\text { Insufficient information to permit judgement of 'low risk' or 'high risk'. }\end{array}$
\end{tabular}

Incomplete outcome data Low risk Comments: No missing outcome data.
(attrition bias)

All outcomes 
Yang 2005 (Continued)

Selective reporting (re- Low risk Comments: The protocol is not available; all outcomes stated in methods othporting bias) er than TESS score reported in results. However, the author reported the number of specific adverse events for the 2 groups.

Other bias Low risk Funding: Guangdong Medical Science and Technology Research Fund Project
(A2002647).

\section{Zhang 2010}

\section{Methods}

Allocation: parallel, randomised

Blind: not stated

Setting: hospital (inpatient), single centre

Country: China

Length of study: 8 weeks

Participants

Diagnosis: treatment-resistant schizophrenia (CCMD-3)

Total $\mathbf{N}$ at randomisation $=246$

Sex: male 109, female 137

Age (years): mean 42.12, SD 11.34

Length of illness (years): mean 7.92, SD 4.98

History: BPRS score > 35; failure after 3 types of antipsychotics (at least 2 types of antipsychotics with different chemical structure) over the last 5 years; with intolerable side effects of antipsychotics; the patient's condition had relapsed or worsened despite use of adequate maintain or preventative treatment.

Exclusion criteria: patients with severe physical or organic brain disease; alcohol or drug abusers; patients with mental retardation; MECT contraindications.

ECT group $(n=123):$ MECT + clozapine

Content: participants who had received clozapine before enrolment continued to use clozapine. Participants who had received other antipsychotics before enrolment needed a 1-week wash-out period and then received clozapine. The initial dose of clozapine was $50 \mathrm{mg} / \mathrm{d}$, increased to 50 to $100 \mathrm{mg} / \mathrm{d}$ within 3 to 4 days. The maximum dose of clozapine was no more than $350 \mathrm{mg} / \mathrm{d}$ when combining with MECT. The ECT device was MECT SpECTrum 5000Q. The clozapine was used at 2 hours after ECT therapy. The traditional bilateral electrode placement was used throughout. The electrical dosing schedule was set using dosage titration therapy.

Frequency: for ECT, 3 times a week in first 4 weeks, then once a week for another 4 weeks; for clozapine, twice daily

Course of treatment (MECT): 15 sessions

Treatment duration: 8 weeks

Control group $(n=123)$ : Clozapine alone

Content: the usage and dosage of clozapine was same as above. The maximum dose was no more than $450 \mathrm{mg} / \mathrm{d}$.

Frequency: twice daily 


Response to treatment: clinically significant response to treatment*
Cognitive functioning: memory assessed by WMS-MQ
Acceptability of treatment: leaving the study early
Mental state: assessed by BPRS
Adverse events: assessed by TESS
*Clinically significant response assessed according to the Chinese evaluation criteria for clinical effica-
cy of mental illness (Weng 1991 , "Concise psychiatry"):
1) cure: reducing rate $\geq 75 \%$;
2) significant improvement: reducing rate between $50 \%$ and $74 \%$;
3) improvement: reducing rate between $25 \%$ and $49 \%$;
4) no clinical response: reducing rate $<25 \%$.
The clinically significant response to treatment was defined as cure + significant improvement.

Contact information: zhangyijie28@163.com; Psychiatric Hospital, Kunming, Yunnan, China

No reply.

\section{Risk of bias}

\begin{tabular}{lll}
\hline Bias & Authors' judgement & Support for judgement \\
\hline $\begin{array}{l}\text { Random sequence genera- } \\
\text { tion (selection bias) }\end{array}$ & Unclear risk & Quote: "...were divided randomly into two groups..." (p.441) \\
& $\begin{array}{l}\text { Comments: The author described a random component in the sequence gen- } \\
\text { eration process, but no details were provided on randomisation method. }\end{array}$
\end{tabular}

\begin{tabular}{lll}
\hline $\begin{array}{l}\text { Allocation concealment } \\
\text { (selection bias) }\end{array}$ & Unclear risk & $\begin{array}{l}\text { Comments: The author did not describe the allocation concealment. Insuffi- } \\
\text { cient information to permit judgement of 'low risk' or 'high risk'. }\end{array}$
\end{tabular}

\begin{tabular}{lll}
\hline $\begin{array}{l}\text { Blinding of participants } \\
\text { and personnel (perfor- }\end{array}$ & High risk & $\begin{array}{l}\text { Comments: The participants and personnel could not be blinded as } 1 \text { group } \\
\text { did not use ECT, and no sham-ECT was used. }\end{array}$
\end{tabular}
mance bias)

All outcomes

Blinding of outcome assessment (detection bias) All outcomes
Unclear risk

Comments: The author did not describe the blinding of outcome assessment. Insufficient information to permit judgement of 'low risk' or 'high risk'.

\begin{tabular}{|c|c|c|}
\hline $\begin{array}{l}\text { Incomplete outcome data } \\
\text { (attrition bias) } \\
\text { All outcomes }\end{array}$ & Low risk & $\begin{array}{l}\text { Comments: } 1 \text { participant }(0.4 \%) \text { in clozapine-alone group left the study early } \\
\text { due to side effects of the drug. }\end{array}$ \\
\hline $\begin{array}{l}\text { Selective reporting (re- } \\
\text { porting bias) }\end{array}$ & Low risk & $\begin{array}{l}\text { Comments: The protocol is not available; all outcomes stated in methods re- } \\
\text { ported in results. }\end{array}$ \\
\hline Other bias & Low risk & None obvious. \\
\hline
\end{tabular}


Allocation: parallel, randomised

Blind: not stated

Setting: hospital (inpatient)

Country: China

Length of study: 8 weeks

\section{Participants}

Diagnosis: treatment-resistant schizophrenia (CCMD-3)

\section{Total $\mathbf{N}$ at randomisation $=84$}

Sex: male 37 , female 47

Age (years): mean 38.4, SD not stated

Length of illness (years): mean 18.6, SD not stated

History: PANSS > 60; failure after 3 types of antipsychotics (at least 2 types of antipsychotics with different chemical structure) over the last 5 years.

Exclusion criteria: patients with severe physical, organic brain disease; alcohol or drug abusers; patients with mental retardation or chronic decline schizophrenia; MECT contraindications.

ECT group $(n=42):$ MECT + olanzapine

Content: the initial dose of olanzapine was $5 \mathrm{mg} / \mathrm{d}$, increased to therapeutic dose (no more than 20 $\mathrm{mg} / \mathrm{d}$ ). The mean dose of olanzapine was $15.3 \mathrm{mg} / \mathrm{d}$. On the day MECT was administered, olanzapine therapy was stopped at noon. The ECT device was Thymatron.

Frequency: for ECT, 3 times a week for the first 4 weeks, then once a week for another 4 weeks; for olanzapine, twice daily

Course of treatment (MECT): 16 sessions

Treatment duration: 8 weeks

Control group $(n=42)$ : Olanzapine alone

Content: the initial dose of olanzapine was $10 \mathrm{mg} / \mathrm{d}$, increased to therapeutic dose (no more than 25 $\mathrm{mg} / \mathrm{d}$ ). The mean dose of olanzapine was $18.5 \mathrm{mg} / \mathrm{d}$.

Frequency: twice daily

Treatment duration: 8 weeks

Response to treatment: clinically significant response to treatment*
Mental state: assessed by PANSS
Adverse events: assessed by TESS
${ }^{*}$ Clinically significant response according to the Chinese evaluation criteria for clinical efficacy of men-
tal illness (Weng 1991, "Concise psychiatry"):
$\begin{array}{ll}\text { 1) cure: reducing rate } \geq 75 \% \text {; } \\ \text { 2) significant improvement: reducing rate between } 50 \% \text { and } 74 \% ; \\ \text { 3) improvement: reducing rate between } 25 \% \text { and } 49 \% ; \\ \text { 4) no clinical response: reducing rate }<25 \% .\end{array}$


Contact information: Brain III Department of People's Liberation Army 261 Hospital, Beijing, China

\section{Risk of bias}

\begin{tabular}{lll}
\hline Bias & Authors' judgement & Support for judgement \\
\hline $\begin{array}{l}\text { Random sequence genera- } \\
\text { tion (selection bias) }\end{array}$ & Unclear risk & Quote: "...were divided randomly into two groups..." (p.141) \\
& $\begin{array}{l}\text { Comments: The author described a random component in the sequence gen- } \\
\text { eration process, but no details were provided on randomisation method. }\end{array}$
\end{tabular}

\begin{tabular}{ll}
\hline $\begin{array}{l}\text { Allocation concealment } \\
\text { (selection bias) }\end{array}$ & Unclear risk
\end{tabular} $\begin{aligned} & \text { Comments: The author did not describe the allocation concealment. Insuffi- } \\
& \text { cient information to permit judgement of 'low risk' or 'high risk'. }\end{aligned}$

\begin{tabular}{|c|c|c|}
\hline $\begin{array}{l}\text { Blinding of participants } \\
\text { and personnel (perfor- }\end{array}$ & High risk & $\begin{array}{l}\text { Comments: The participants and personnel could not be blinded as } 1 \text { group } \\
\text { did not use ECT, and no sham-ECT was used. }\end{array}$ \\
\hline
\end{tabular}
mance bias)

All outcomes

Blinding of outcome as- Unclear risk sessment (detection bias) All outcomes
Comments: The author did not describe the blinding of outcome assessment. Insufficient information to permit judgement of 'low risk' or 'high risk'.

Incomplete outcome data Low risk Comments: No missing outcome data.
(attrition bias)

(attrition bias)

All outcomes

\begin{tabular}{ll}
\hline $\begin{array}{l}\text { Selective reporting (re- } \\
\text { porting bias) }\end{array}$ & Low risk \\
\end{tabular}

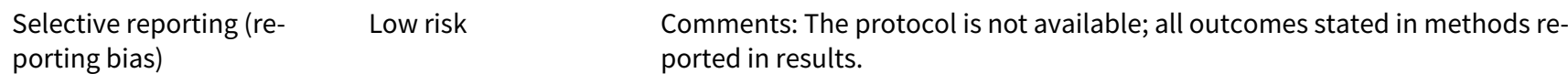

Other bias Low risk None obvious.

BPRS: Brief Psychiatric Rating Scale (lower is better); CCMD: Chinese Classification of Mental Disorders; CGI-I: Clinical Global Impression - Improvement scale (lower is better); CGI-S: Clinical Global Impression - Severity scale (lower is better); DSM: Diagnostic and Statistical Manual of Mental Disorders; ECT: electroconvulsive therapy; GAF: Global Assessment of Functioning (higher is better); HAM-D: Hamilton Depression Rating Scale (lower is better); ICD-10: International Statistical Classification of Diseases and Related Health Problems 10th Revision; ITT: intention-to-treat; MECT: modified electroconvulsive therapy; MMSE: Mini-Mental State Examination (higher is better); PANSS: Positive and Negative Syndrome Scale (lower is better); SANS: Scale for Assessment of Negative Symptoms (lower is better); SAPS: Scale for Assessment of Positive Symptoms (lower is better); SD: standard deviation; TESS: Treatment Emergent Symptom Scale (lower is better); WCST: Wisconsin Card Sorting Testing (lower is better); WMS: Wechsler Memory Scale (higher is better); WMS-MQ: Wechsler Memory Scale - Memory quotient (higher is better)

\section{Characteristics of excluded studies [ordered by study ID]}

\begin{tabular}{ll}
\hline Study & Reason for exclusion \\
\hline Abraham 1987 & The participants had schizophrenia, but not with a diagnosis of treatment-resistant. \\
\hline Abrams 1967 & The participants had schizophrenia, but not with a diagnosis of treatment-resistant. \\
\hline Agarwal 1985 & The participants had schizophrenia, but not with a diagnosis of treatment-resistant. \\
\hline Arato 1980 & Not RCT \\
\hline
\end{tabular}




\begin{tabular}{|c|c|}
\hline Study & Reason for exclusion \\
\hline Bagadia 1981 & $\begin{array}{l}\text { ECT combined with other interventions, but other interventions were not the same between } \\
\text { groups: ECT + placebo versus simulated ECT + imipramine }\end{array}$ \\
\hline Bagadia 1988 & The participants had schizophrenia, but not with a diagnosis of treatment-resistant. \\
\hline Baker 1958 & The participants had schizophrenia, but not with a diagnosis of treatment-resistant. \\
\hline Baker 1960 & The participants had schizophrenia, but not with a diagnosis of treatment-resistant. \\
\hline Baker 1960a & The participants had schizophrenia, but not with a diagnosis of treatment-resistant. \\
\hline Bhatia 1987 & Not RCT \\
\hline Brandon 1985 & The participants had schizophrenia, but not with a diagnosis of treatment-resistant. \\
\hline Brill 1959 & Not whole population had schizophrenia or related disorder. \\
\hline Chatterjee 1980 & The participants had schizophrenia, but not with a diagnosis of treatment-resistant. \\
\hline ChiCTR-OPC-14005339 & Not RCT \\
\hline d'Elia 1970 & Not whole population had schizophrenia or related disorder. \\
\hline Doongaji 1973 & The participants had schizophrenia, but not with a diagnosis of treatment-resistant. \\
\hline El Islam 1970 & The participants had schizophrenia, but not with a diagnosis of treatment-resistant. \\
\hline Gan 2017 & The participants had schizophrenia, but not with a diagnosis of treatment-resistant. \\
\hline Gander 1967 & Not whole population had schizophrenia or related disorder. \\
\hline Gangadhar 2000 & The participants had schizophrenia, but not with a diagnosis of treatment-resistant. \\
\hline Girish 2003 & Not whole population had schizophrenia or related disorder. \\
\hline Hargreaves 1972 & Not whole population had schizophrenia or related disorder. \\
\hline He 2001 & ECT used in both groups: ECT advantage side versus non-advantage side. \\
\hline Heath 1964 & Not RCT \\
\hline Janakiramaiah 1981 & The participants had schizophrenia, but not with a diagnosis of treatment-resistant. \\
\hline Janakiramaiah 1982 & The participants had schizophrenia, but not with a diagnosis of treatment-resistant. \\
\hline Krystal 1993 & Not whole population had schizophrenia or related disorder. \\
\hline Laurell 1970 & Not whole population had schizophrenia or related disorder. \\
\hline Laurell 1970a & Not whole population had schizophrenia or related disorder. \\
\hline Marjerrison 1975 & The participants had schizophrenia, but not with a diagnosis of treatment-resistant. \\
\hline Miller 1953 & The participants had schizophrenia, but not with a diagnosis of treatment-resistant. \\
\hline
\end{tabular}




\begin{tabular}{|c|c|}
\hline Study & Reason for exclusion \\
\hline Naidoo 1956 & The participants had schizophrenia, but not with a diagnosis of treatment-resistant. \\
\hline Natani 1983 & The participants had schizophrenia, but not with a diagnosis of treatment-resistant. \\
\hline NCT02159001 & ECT used in both groups: ECT treatment right after recruitment versus ECT after 4-week period. \\
\hline Rami 2008 & The participants had schizophrenia, but not with a diagnosis of treatment-resistant. \\
\hline Reichert 1976 & Not whole population had schizophrenia or related disorder. \\
\hline Sarkar 1994 & The participants had schizophrenia, but not with a diagnosis of treatment-resistant. \\
\hline Small 1968 & The participants had schizophrenia, but not with a diagnosis of treatment-resistant. \\
\hline Smith 1967 & Not RCT \\
\hline Stenback 1957 & ECT used in both groups: usual ECT versus block ECT. \\
\hline Swoboda 2001 & Not RCT \\
\hline Taylor 1980 & The participants had schizophrenia, but not with a diagnosis of treatment-resistant. \\
\hline Ukpong 2002 & The participants had schizophrenia, but not with a diagnosis of treatment-resistant. \\
\hline Ulett 1956 & Not whole population had schizophrenia or related disorder. \\
\hline Ungvari 1981 & Language other than English or Chinese \\
\hline Wessels 1971 & The participants had schizophrenia, but not with a diagnosis of treatment-resistant. \\
\hline Xue 1985 & The participants had schizophrenia, but not with a diagnosis of treatment-resistant. \\
\hline Zadeh 2006 & Language other than English or Chinese \\
\hline Zhou 2003 & Not RCT \\
\hline
\end{tabular}

ECT: electroconvulsive therapy; RCT: randomised controlled trial

Characteristics of studies awaiting assessment [ordered by study ID]

Chen 2006

Methods

Allocation: parallel, randomised

Blind: awaiting full text

Country: China

Length of study: awaiting full text

$\begin{array}{ll}\text { Participants } & \text { Diagnosis: schizophrenia } \\ \text { Total } \mathbf{N} \text { at randomisation }=83 \\ \text { Sex: awaiting full text } \\ \text { Age (years): awaiting full text }\end{array}$


Chen 2006 (Continued)

Length of illness (years): awaiting full text

Inclusion criteria: awaiting full text

Exclusion criteria: awaiting full text

\begin{tabular}{ll}
\hline Interventions & $\begin{array}{l}\text { ECT group: Electric shock with small doses of antipsychotic drug treatment } \\
\text { Control group: Antipsychotic treatment only }\end{array}$ \\
\hline Outcomes & Refuse drugs, depression, behavioural disorders \\
\hline Notes & $\begin{array}{l}\text { Awaiting classification due to unclear if schizophrenia is treatment-resistant and unclear informa- } \\
\text { tion on comparison. }\end{array}$ \\
Contact information: Not available
\end{tabular}

Melzer 2015

\begin{tabular}{|c|c|}
\hline Methods & $\begin{array}{l}\text { Allocation: parallel, randomised } \\
\text { Blind: double-blind } \\
\text { Country: Brazil } \\
\text { Length of study: awaiting full text }\end{array}$ \\
\hline Participants & $\begin{array}{l}\text { Diagnosis: super-refractory schizophrenia } \\
\text { Total } N \text { at randomisation }=20 \\
\text { Sex: men and women } \\
\text { Age (years): range } 18 \text { to } 55 \\
\text { Length of illness (years): awaiting full text } \\
\text { Inclusion criteria: } \\
\text { (1) DSM-IV-TR criteria for schizophrenia } \\
\text { (2) Age between } 18 \text { and } 55 \text { years, men and women } \\
\text { (3) All patients were on clozapine treatment for at least } 6 \text { months, with or without add-on therapy, } \\
\text { with unsatisfactory response, defined as CGI } \geq 4 \text {, and PANSS total } \geq 60 \text { at baseline } \\
\text { Exclusion criteria: awaiting full text }\end{array}$ \\
\hline Interventions & $\begin{array}{l}\text { ECT group: } E C T \\
\text { Control group: Sham-ECT }\end{array}$ \\
\hline Outcomes & PANSS (and its sub scales - positive, negative, and general psychopathology) and CGI \\
\hline Notes & $\begin{array}{l}\text { Awaiting classification due to no full text. } \\
\text { Contact information: Not available }\end{array}$ \\
\hline
\end{tabular}


Nicholas 1996

\begin{tabular}{|c|c|}
\hline Methods & $\begin{array}{l}\text { Allocation: awaiting full text } \\
\text { Blind: double-blind } \\
\text { Country: USA } \\
\text { Length of study: awaiting full text }\end{array}$ \\
\hline Participants & $\begin{array}{l}\text { Diagnosis: awaiting full text } \\
\text { Total } \mathbf{N} \text { at randomisation: awaiting full text } \\
\text { Sex: awaiting full text } \\
\text { Age (years): awaiting full text } \\
\text { Length of illness (years): awaiting full text } \\
\text { Inclusion criteria: awaiting full text } \\
\text { Exclusion criteria: awaiting full text }\end{array}$ \\
\hline Interventions & $\begin{array}{l}\text { ECT group: Right unilateral of threshold level of ECT } \\
\text { Control group: Bitemporal of threshold level of ECT } \\
\text { Group 3: Bifrontal of threshold level of ECT }\end{array}$ \\
\hline Outcomes & $\begin{array}{l}\text { Response to treatment, the relationship between electrical dose, seizure threshold, and clinica } \\
\text { outcome and cognitive }\end{array}$ \\
\hline Notes & $\begin{array}{l}\text { Awaiting classification due to unclear diagnosis and study design. } \\
\text { Contact information: Not available }\end{array}$ \\
\hline
\end{tabular}

$\begin{array}{ll}\text { Methods } & \text { Allocation: randomised } \\ \text { Blind: awaiting full text } \\ \text { Country: Russia } \\ \text { Length of study: } 10 \text { weeks } \\ \text { Diagnosis: treatment-resistant schizophrenia (DSM-IV criteria) } \\ \text { Total N at randomisation }=40 \\ \text { Sex: awaiting full text } \\ \text { Age (years): range } 18 \text { to } 60 \\ \text { Length of illness (years): awaiting full text } \\ \text { Inclusion criteria: resistance was defined as absence of response (less than } 20 \% \text { reduction of total } \\ \text { PANSS score) }\end{array}$

Exclusion criteria: awaiting full text 
Oleneva 2005 (Continued)

$$
\text { Control group: Olanzapine alone }
$$

\begin{tabular}{ll}
\hline Outcomes & Response (considered as a PANSS score more than 20\%) \\
\hline Notes & Awaiting classification due to no full text. \\
& Contact information: Not available \\
\hline
\end{tabular}

Peyman 1956

\begin{tabular}{ll}
\hline Methods & Allocation: awaiting full text \\
Blind: awaiting full text \\
Country: awaiting full text \\
Length of study: 6 months \\
\hline
\end{tabular}

\begin{tabular}{ll}
\hline Participants & Diagnosis: chronic schizophrenia reactions \\
Total $\mathbf{N}$ at randomisation $=32$ \\
Sex: female 32 \\
Age (years): awaiting full text \\
Length of illness (years): awaiting full text \\
Inclusion criteria: awaiting full text \\
Exclusion criteria: awaiting full text \\
ECT group: Group psychotherapy \\
Control group: Electric shock therapy \\
Group 3: Group psychotherapy + electric shock therapy \\
Group 4: Control (no treatment) \\
Wechsler-Bellevue Intelligence Scale, Rorschach, and Bender-Gestalt Tests \\
\hline Awaiting classification due to unclear if schizophrenia was treatment-resistant and unclear study \\
design. \\
Contact information: Not available
\end{tabular}

Sackeim 2000

Methods Allocation: parallel, randomised

Blind: double-blind

Country: awaiting full text

Length of study: awaiting full text

Participants Diagnosis: awaiting full text


Total $\mathbf{N}$ at randomisation: awaiting full text

Sex: awaiting full text

Age (years): awaiting full text

Length of illness (years): awaiting full text

Inclusion criteria: awaiting full text

Exclusion criteria: awaiting full text

Interventions

ECT group: Right unilateral ECT

Control group: Bilateral ECT

\begin{tabular}{ll}
\hline Outcomes & Cognitive side effects \\
\hline Notes & Awaiting classification due to unclear diagnosis.
\end{tabular}

Contact information: Not available

CGI: Clinical Global Impression (lower is better); ECT: electroconvulsive therapy; PANSS: Positive and Negative Syndrome Scale (lower is better)

Characteristics of ongoing studies [ordered by study ID]

NCT00042224

Trial name or title Electroconvulsive therapy in clozapine refractory schizophrenia

Methods Allocation: parallel, randomised

Blind: single (outcome assessor)

Country: USA

Length of study: 8 weeks

Participants Diagnosis: schizophrenia (DSM-IV)

Total $\mathbf{N}$ at randomisation $=39$

Sex: both

Age (years): range 18 to 60

Length of illness (years): range $>2$

\section{Inclusion criteria:}

- diagnosis of schizophrenia according to DSM-IV criteria

- duration of illness 2 years or greater

- resistance to at least 2 antipsychotics

- clozapine resistance

- capacity to give informed consent

- for women of childbearing capacity, a negative pregnancy test and patient agreement to use a medically accepted form of contraception

- Brief Psychiatric Rating Scale score of at least a 4 on 1 of the 4 psychotic items on the psychotic sub scale or a score of 12 on these 4 items combined

- CGI-severity rating of at least moderate (score of 4) 
NCT00042224 (Continued)

- receiving at least two $400 \mathrm{mg}$ doses of chlorpromazine equivalents for at least 4 weeks (may include newer antipsychotics)

- having substantial psychotic symptoms despite at least 12 weeks of treatment (at least 8 weeks at a consistent dose)

\section{Exclusion criteria:}

- schizoaffective disorder

- bipolar disorder

- current affective episode

- ECT within the past 6 months

- history of epilepsy

- severe neurological or systemic disorder that could significantly affect cognition, behaviour, or mental status (other than tardive dyskinesia or neuroleptic-induced parkinsonism)

- psychoactive substance dependence (other than nicotine or caffeine) within 1 month prior to entering the study

- a score of less than 18 on the 24-item HAM-D

- clinical determination that mood stabilisers were necessary and therefore could not be discontinued

- pregnancy

- affective disorders and prominent depressive symptoms because ECT is well-known to be effective in such situations, and we wanted to avoid contamination of our results by improvement solely driven by the treatment of the affective symptoms

Control group: Clozapine for 8 weeks

\begin{tabular}{|c|c|}
\hline Outcomes & Response rate ${ }^{\star}$ \\
\hline Starting date & December 2000 \\
\hline Contact information & George Petrides, Northwell Health \\
\hline Notes & $\begin{array}{l}\text { No results have been published. } \\
\text { *Response is defined as } 40 \% \text { reduction of symptoms in the psychotic symptom sub scale (halluci- } \\
\text { natory behaviour, suspiciousness, conceptual disorganisation, and unusual thought content) of } \\
\text { the BPRS at the end of the } 8 \text {-week study. BPRS assesses psychotic symptoms on an } 18 \text {-item scale. } \\
\text { The severity of each item is rated on a continuous scale from } 1 \text { to } 7 \text {, with } 1 \text { being the least severe } \\
\text { and } 7 \text { being the most severe. Patients included in the study had at baseline at least a moderate } \\
\text { score of } 4 \text { on } 1 \text { of the } 4 \text { psychotic items on the psychotic symptom sub scale or a score of } 12 \text { on all } 4 \\
\text { of these items combined (ranges } 4 \text { to } 28 \text {, with higher scores indicative of greater severity). A reduc- } \\
\text { tion of symptoms would be a sub scale score that is } 40 \% \text { less than participants' baseline score. If } \\
\text { a participant enters the study with a sub scale score of } 15 \text {, to be considered a responder (at least a } \\
40 \% \text { reduction in symptoms score) his/her score must decrease by at least } 6 \text { points and be } 9 \text { or less. }\end{array}$ \\
\hline
\end{tabular}
vulsive therapy (ECT) (TCRS)

Methods

Allocation: parallel, randomised

Blind: open-label

Country: Thailand 
NCT00753051 (Continued)

Length of study: 24 weeks

\begin{tabular}{|c|c|}
\hline Participants & $\begin{array}{l}\text { Diagnosis: schizophrenia (DSM-IV-TR or ICD-10) } \\
\text { Total } \mathbf{N} \text { at randomisation = } 18 \\
\text { Sex: both } \\
\text { Age (years): range > } 18 \\
\text { Length of illness (years): not stated } \\
\text { Inclusion criteria } \\
\text { - people with schizophrenia diagnosed with Schedules for Clinical Assessment in Neuropsychiatry } \\
\text { (SCAN) in accordance with DSM-IV-TR or ICD-10 } \\
\text { - resistant to at least } 6 \text { weeks of clozapine treatment in therapeutic dosage } \\
\text { Exclusion criteria: } \\
\text { - patient does not volunteer him or herself } \\
\text { - patient has epilepsy, cardiac disease, autistic disorder, or is illiterate } \\
\text { - patient is deaf, blind, obstructing good communication }\end{array}$ \\
\hline Interventions & $\begin{array}{l}\text { ECT group: Clozapine as the main agent adjuncted by ECT } \\
\text { Control group: Clozapine as the main agent adjuncted by haloperidol }\end{array}$ \\
\hline Outcomes & PANSS, HAM-D, CGI, AIMS scores \\
\hline Starting date & June 2008 \\
\hline Contact information & Suchat Paholpak, Khon Kaen University \\
\hline Notes & No results have been published. \\
\hline
\end{tabular}

Blind: double (participant, investigator)

Country: China

Length of study: 12 weeks

\section{Participants}

Diagnosis: schizophrenia (DSM-V)

\section{Estimated $\mathbf{N}$ at randomisation $=150$}

Sex: both

Age (years): range 18 to 60

Length of illness (years): not stated

\section{Inclusion criteria}


NCT02926976 (Continued)

- diagnosis of schizophrenia according to DSM-V

- 18 to 60 years old

- 2 prior failed treatment trials with 2 different antipsychotics at doses of at least $600 \mathrm{mg} /$ day chlorpromazine equivalents, each of at least 6 weeks duration

- signed an informed consent

\section{Exclusion criteria:}

- patients diagnosed according to DSM-V for substance abuse, development delayed

- suffering from serious physical disease and cannot accept the treatment

- MST contraindications: intracranial metal substance, heart pacemakers, cochlear implants, intracranial pressure

- allergic to risperidone, aripiprazole, or sodium valproate

- participated as a clinical subject within 30 days

- pregnancy or lactation

ECT group: Risperidone with clozapine. Risperidone; dosage form: $1 \mathrm{mg}$; dosage and frequency:2.0 $6.0 \mathrm{mg} / \mathrm{d}$; duration: 3 months.

Control group: Aripiprazole with clozapine. Aripiprazole; dosage form: $5 \mathrm{mg}$; dosage and frequency:10 $30 \mathrm{mg} /$ day; duration: 3 months.

Group 3: Sodium valproate with clozapine. Sodium valproate; dosage form: $250 \mathrm{mg}$; dosage and frequency:500 $1000 \mathrm{mg} /$ day; duration: 3 months.

Group 4: MECT with clozapine. 10 times MECT for 4 weeks.

Group 5: MST with clozapine. 10 times MST for 4 weeks.

\begin{tabular}{ll}
\hline Outcomes & PANSS, CGI, SAS, AIMS scores \\
\hline Starting date & November 2016 \\
\hline Contact information & Dengtang Liu \\
& $+862164387250-73775$ \\
& erliu110@126.com \\
\hline Notes & Not yet recruiting. \\
\hline
\end{tabular}

AIMS: Abnormal Involuntary Movement Scale; BPRS: Brief Psychiatric Rating Scale (lower is better); CGI: Clinical Global Impression; DSM: Diagnostic and Statistical Manual of Mental Disorders; ECT: electroconvulsive therapy; HAM-D: Hamilton Depression Rating Scale; ICD-10: International Statistical Classification of Diseases and Related Health Problems 10th Revision; MECT: modified electroconvulsive therapy; MST: magnetic seizure therapy; PANSS: Positive and Negative Syndrome Scale; SAS: Simpson-Angus Scale

\section{DATA AND ANALYSES}

Comparison 1. ECT plus standard care versus sham-ECT plus standard care

\begin{tabular}{lllll}
\hline Outcome or subgroup title & $\begin{array}{l}\text { No. of } \\
\text { studies }\end{array}$ & $\begin{array}{l}\text { No. of } \\
\text { partici- } \\
\text { pants }\end{array}$ & Statistical method & Effect size \\
\hline $\begin{array}{l}1 \text { Mental state - total score (BPRS, high = poor) } \\
\text { - short term }\end{array}$ & 1 & 25 & Mean Difference (IV, Fixed, 95\% Cl) & $3.60[-3.69,10.89]$ \\
\hline
\end{tabular}




\begin{tabular}{llllll}
\hline Outcome or subgroup title & $\begin{array}{l}\text { No. of } \\
\text { studies }\end{array}$ & $\begin{array}{l}\text { No. of } \\
\text { partici- } \\
\text { pants }\end{array}$ & Statistical method & Effect size \\
\hline $\begin{array}{l}2 \text { Service use - number readmitted - short } \\
\text { term }\end{array}$ & 1 & 25 & Risk Ratio (M-H, Fixed, 95\% Cl) & $0.29[0.10,0.85]$ \\
\hline
\end{tabular}

Analysis 1.1. Comparison 1 ECT plus standard care versus sham-ECT plus standard care, Outcome 1 Mental state - total score (BPRS, high = poor) - short term.

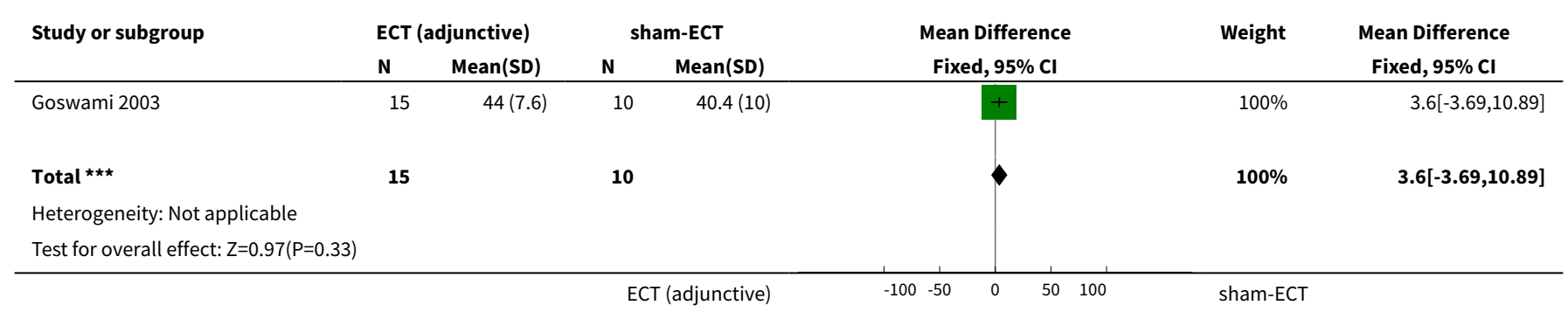

Analysis 1.2. Comparison 1 ECT plus standard care versus sham-ECT plus standard care, Outcome 2 Service use - number readmitted - short term.

\begin{tabular}{|c|c|c|c|c|c|c|}
\hline \multirow[t]{2}{*}{ Study or subgroup } & $\begin{array}{l}\text { ECT (ad- } \\
\text { junctive) }\end{array}$ & \multicolumn{2}{|l|}{ sham-ECT } & Risk Ratio & \multirow[t]{2}{*}{ Weight } & \multirow{2}{*}{$\begin{array}{c}\text { Risk Ratio } \\
\text { M-H, Fixed, } 95 \% \mathrm{Cl}\end{array}$} \\
\hline & $n / N$ & $n / N$ & & M-H, Fixed, 95\% Cl & & \\
\hline Goswami 2003 & $3 / 15$ & $7 / 10$ & & - & $100 \%$ & $0.29[0.1,0.85]$ \\
\hline Total $(95 \% \mathrm{Cl})$ & 15 & 10 & & & $100 \%$ & $0.29[0.1,0.85]$ \\
\hline \multicolumn{7}{|c|}{ Total events: 3 (ECT (adjunctive)), 7 (sham-ECT) } \\
\hline \multicolumn{7}{|c|}{ Test for overall effect: $Z=2.25(P=0.02)$} \\
\hline & & ECT (adjunctive) & 0.001 & 0.1 & 1000 sham-ECT & \\
\hline
\end{tabular}

Comparison 2. ECT plus standard care versus antipsychotics plus standard care

\begin{tabular}{|c|c|c|c|c|}
\hline Outcome or subgroup title & $\begin{array}{l}\text { No. of } \\
\text { studies }\end{array}$ & $\begin{array}{l}\text { No. of } \\
\text { partici- } \\
\text { pants }\end{array}$ & Statistical method & Effect size \\
\hline $\begin{array}{l}1 \text { Response to treatment - } \\
\text { clinically important response } \\
\text { (BPRS reducing rate } \geq 50 \% \text { ) - } \\
\text { medium term }\end{array}$ & 1 & 162 & Risk Ratio (M-H, Fixed, 95\% Cl) & $1.23[0.95,1.58]$ \\
\hline $\begin{array}{l}2 \text { Mental state - total score } \\
\text { (BPRS, high = poor) - short } \\
\text { term }\end{array}$ & 1 & 162 & Mean Difference (IV, Fixed, 95\% CI) & $-5.20[-7.93,-2.47]$ \\
\hline
\end{tabular}




\begin{tabular}{|c|c|c|c|c|}
\hline Outcome or subgroup title & $\begin{array}{l}\text { No. of } \\
\text { studies }\end{array}$ & $\begin{array}{l}\text { No. of } \\
\text { partici- } \\
\text { pants }\end{array}$ & Statistical method & Effect size \\
\hline $\begin{array}{l}3 \text { Mental state - total score } \\
\text { (BPRS, high = poor) - medium } \\
\text { term (skewed data) }\end{array}$ & & & Other data & No numeric data \\
\hline $\begin{array}{l}4 \text { Mental state - specific symp- } \\
\text { tom score (BPRS, high = poor) } \\
\text { - short term }\end{array}$ & 1 & & Mean Difference (IV, Fixed, 95\% CI) & Subtotals only \\
\hline 4.1 Anxiety and depression & 1 & 162 & Mean Difference (IV, Fixed, 95\% CI) & $-0.60[-1.12,-0.08]$ \\
\hline 4.2 Lack of vitality & 1 & 162 & Mean Difference (IV, Fixed, 95\% CI) & $-2.20[-2.74,-1.66]$ \\
\hline 4.3 Thought disturbance & 1 & 162 & Mean Difference (IV, Fixed, 95\% CI) & $-0.70[-1.32,-0.08]$ \\
\hline 4.4 Agitation & 1 & 162 & Mean Difference (IV, Fixed, 95\% CI) & $-0.70[-0.82,-0.58]$ \\
\hline 4.5 Hostile suspiciousness & 1 & 162 & Mean Difference (IV, Fixed, 95\% CI) & $-1.0[-1.56,-0.44]$ \\
\hline $\begin{array}{l}5 \text { Mental state - specific symp- } \\
\text { tom score (BPRS, high = poor) - } \\
\text { medium term }\end{array}$ & 1 & & Mean Difference (IV, Fixed, 95\% CI) & Subtotals only \\
\hline 5.1 Anxiety and depression & 1 & 162 & Mean Difference (IV, Fixed, 95\% CI) & $-1.50[-1.73,-1.27]$ \\
\hline 5.2 Lack of vitality & 1 & 162 & Mean Difference (IV, Fixed, 95\% CI) & $-1.20[-1.47,-0.93]$ \\
\hline 5.3 Thought disturbance & 1 & 162 & Mean Difference (IV, Fixed, 95\% CI) & $-1.70[-2.12,-1.28]$ \\
\hline 5.4 Agitation & 1 & 162 & Mean Difference (IV, Fixed, 95\% CI) & $-1.60[-1.79,-1.41]$ \\
\hline 5.5 Hostile suspiciousness & 1 & 162 & Mean Difference (IV, Fixed, 95\% CI) & $-2.80[-3.14,-2.46]$ \\
\hline $\begin{array}{l}6 \text { Adverse events - total score } \\
\text { (TESS, high = poor) }\end{array}$ & 1 & & Mean Difference (IV, Fixed, 95\% CI) & Subtotals only \\
\hline 6.1 Short term & 1 & 162 & Mean Difference (IV, Fixed, 95\% CI) & $-0.40[-0.91,0.11]$ \\
\hline 6.2 Medium term & 1 & 162 & Mean Difference (IV, Fixed, 95\% CI) & $-1.1[-1.40,-0.80]$ \\
\hline
\end{tabular}

\section{Analysis 2.1. Comparison 2 ECT plus standard care versus antipsychotics plus standard care, Outcome 1 Response to treatment - clinically important response (BPRS reducing rate $\geq 50 \%$ ) - medium term.}

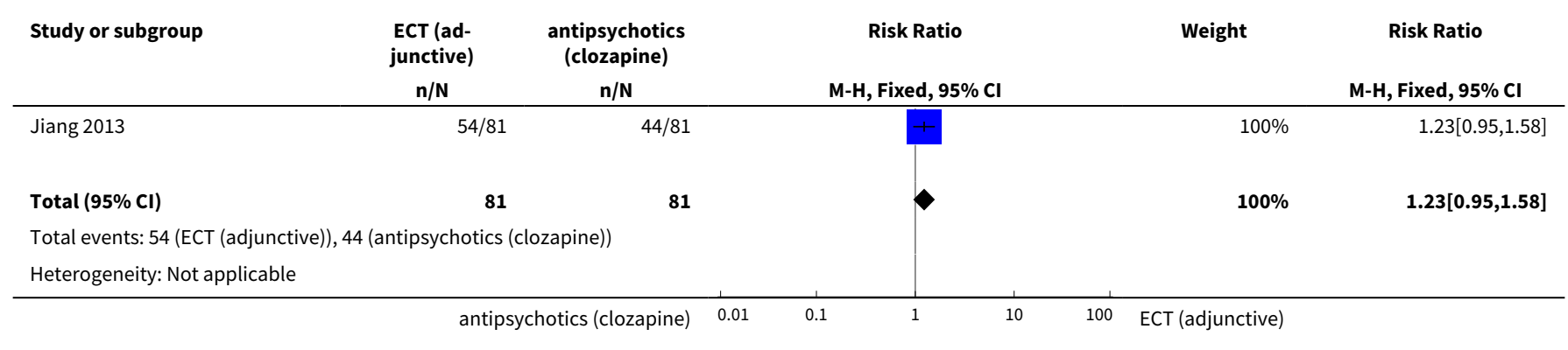




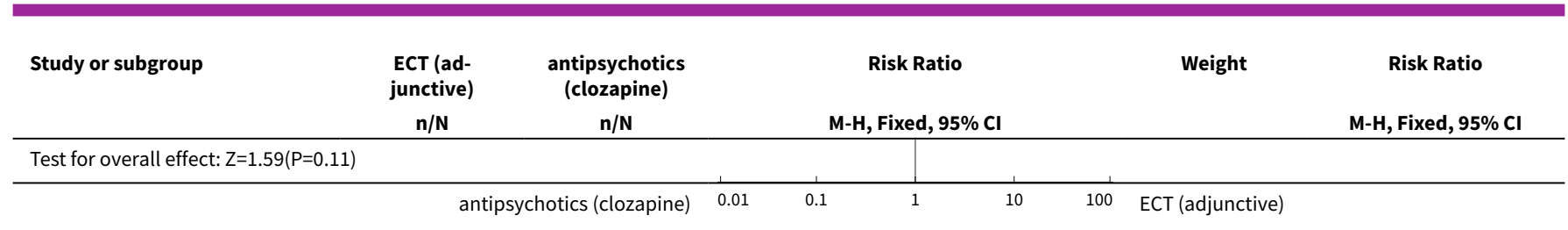

Analysis 2.2. Comparison 2 ECT plus standard care versus antipsychotics plus standard care, Outcome 2 Mental state - total score (BPRS, high = poor) - short term.

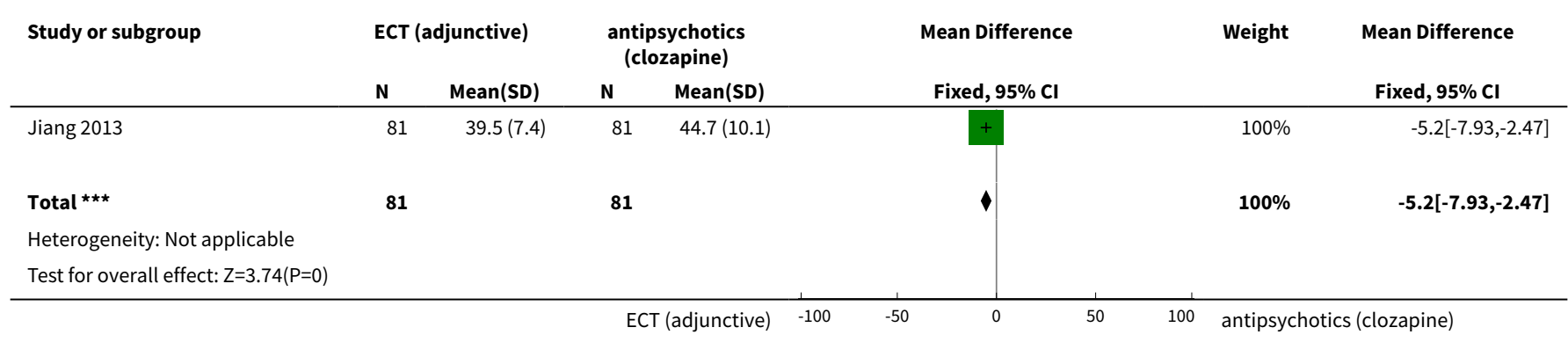

Analysis 2.3. Comparison 2 ECT plus standard care versus antipsychotics plus standard care, Outcome 3 Mental state - total score (BPRS, high = poor) - medium term (skewed data).

\begin{tabular}{|c|c|c|c|c|}
\hline \multicolumn{5}{|c|}{ Mental state - total score $(B P R S$, high $=$ poor) - medium term (skewed data) } \\
\hline Study & Interventions & Mean & SD & $\mathbf{N}$ \\
\hline Jiang 2013 & MECT + ziprasidone & 20.8 & 4.1 & 81 \\
\hline Jiang 2013 & Clozapine + ziprasidone & 29.6 & 9.7 & 81 \\
\hline
\end{tabular}

Analysis 2.4. Comparison $2 \mathrm{ECT}$ plus standard care versus antipsychotics plus standard care, Outcome 4 Mental state - specific symptom score (BPRS, high = poor) - short term.

\begin{tabular}{|c|c|c|c|c|c|c|c|}
\hline \multirow[t]{2}{*}{ Study or subgroup } & \multicolumn{2}{|c|}{ ECT (adjunctive) } & \multicolumn{2}{|c|}{$\begin{array}{l}\text { antipsychotics } \\
\text { (clozapine) }\end{array}$} & \multirow{2}{*}{$\begin{array}{c}\text { Mean Difference } \\
\text { Fixed, } 95 \% \mathrm{Cl} \\
\end{array}$} & \multirow[t]{2}{*}{ Weight } & \multirow{2}{*}{$\begin{array}{l}\text { Mean Difference } \\
\text { Fixed, } 95 \% \mathrm{Cl}\end{array}$} \\
\hline & $\mathbf{N}$ & Mean(SD) & $\mathbf{N}$ & $\operatorname{Mean}(\mathrm{SD})$ & & & \\
\hline \multicolumn{8}{|c|}{ 2.4.1 Anxiety and depression } \\
\hline Jiang 2013 & 81 & $6.9(1.1)$ & 81 & $7.5(2.1)$ & + & $100 \%$ & $-0.6[-1.12,-0.08]$ \\
\hline Subtotal $\star \star \star$ & 81 & & 81 & & 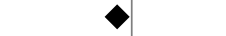 & $100 \%$ & $-0.6[-1.12,-0.08]$ \\
\hline \multicolumn{8}{|c|}{ Heterogeneity: Not applicable } \\
\hline \multicolumn{8}{|c|}{ Test for overall effect: $\mathrm{Z}=2.28(\mathrm{P}=0.02)$} \\
\hline \multicolumn{8}{|l|}{ 2.4.2 Lack of vitality } \\
\hline Jiang 2013 & 81 & $8.5(0.7)$ & 81 & $10.7(2.4)$ & - & $100 \%$ & $-2.2[-2.74,-1.66]$ \\
\hline 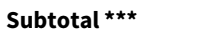 & 81 & & 81 & & $\diamond$ & $100 \%$ & $-2.2[-2.74,-1.66]$ \\
\hline \multicolumn{8}{|c|}{ Heterogeneity: Not applicable } \\
\hline \multicolumn{8}{|c|}{ Test for overall effect: $Z=7.92(P<0.0001)$} \\
\hline \multicolumn{8}{|c|}{ 2.4.3 Thought disturbance } \\
\hline Jiang 2013 & 81 & $10.8(2.1)$ & 81 & $11.5(1.9)$ & & $100 \%$ & $-0.7[-1.32,-0.08]$ \\
\hline Subtotal $* \star \star$ & 81 & & 81 & & $>$ & $100 \%$ & $-0.7[-1.32,-0.08]$ \\
\hline
\end{tabular}




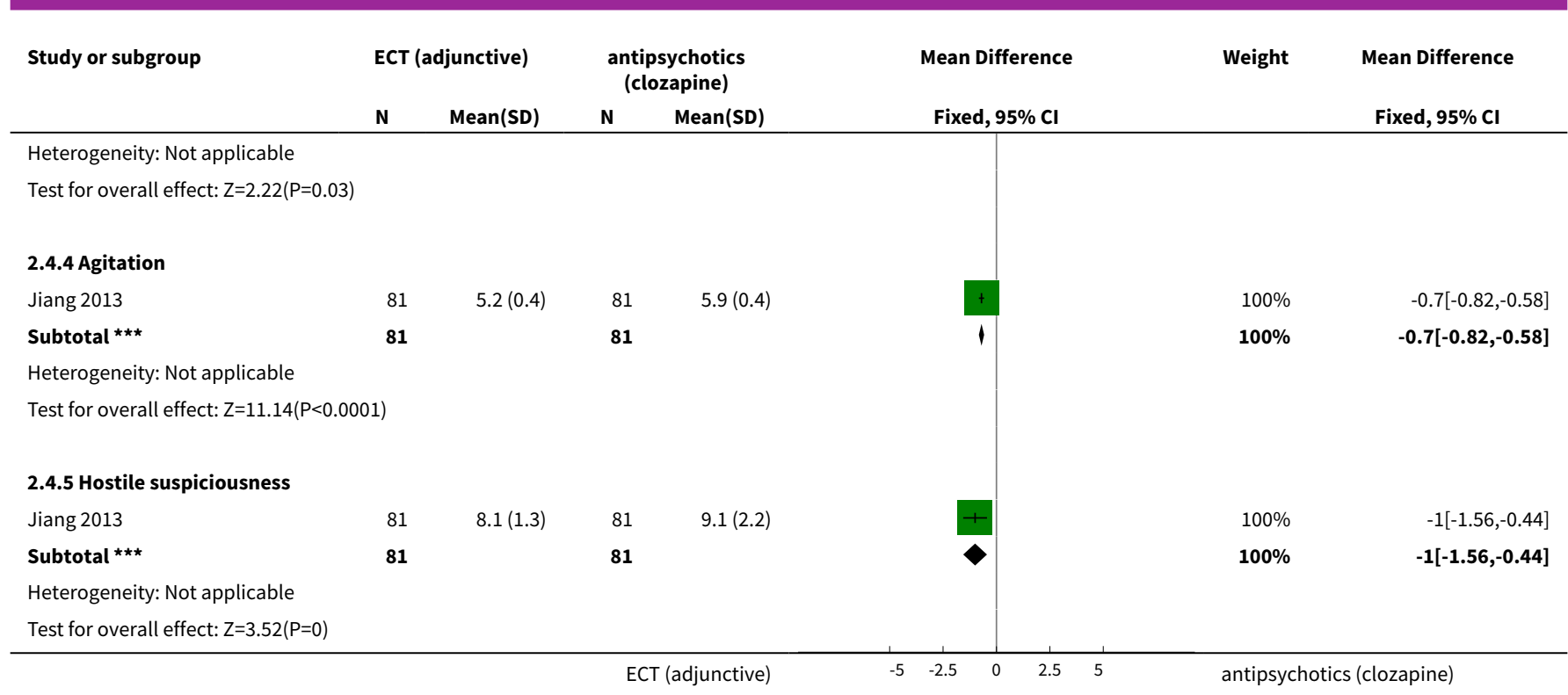

Analysis 2.5. Comparison 2 ECT plus standard care versus antipsychotics plus standard care, Outcome 5 Mental state - specific symptom score (BPRS, high = poor) - medium term.

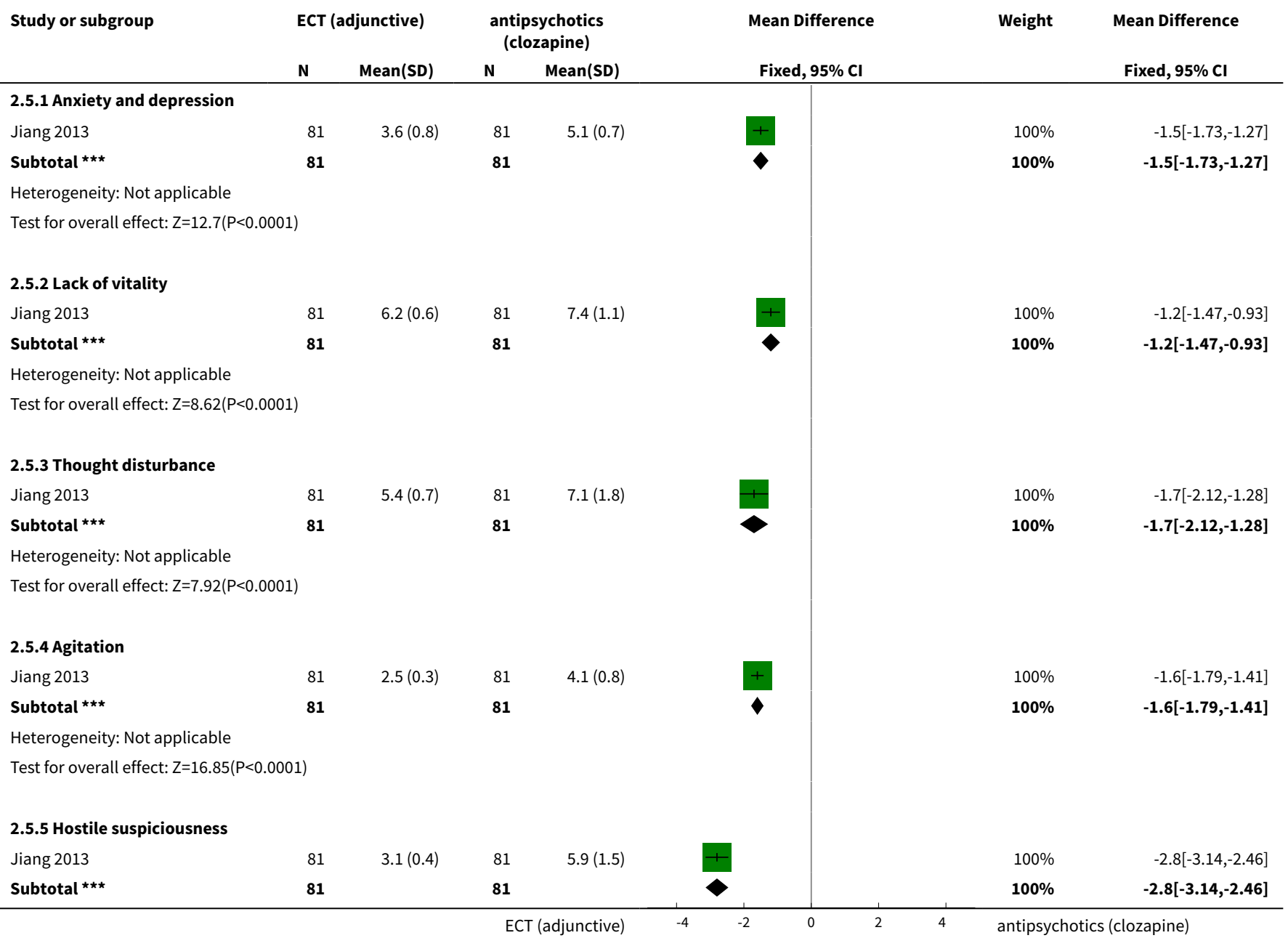




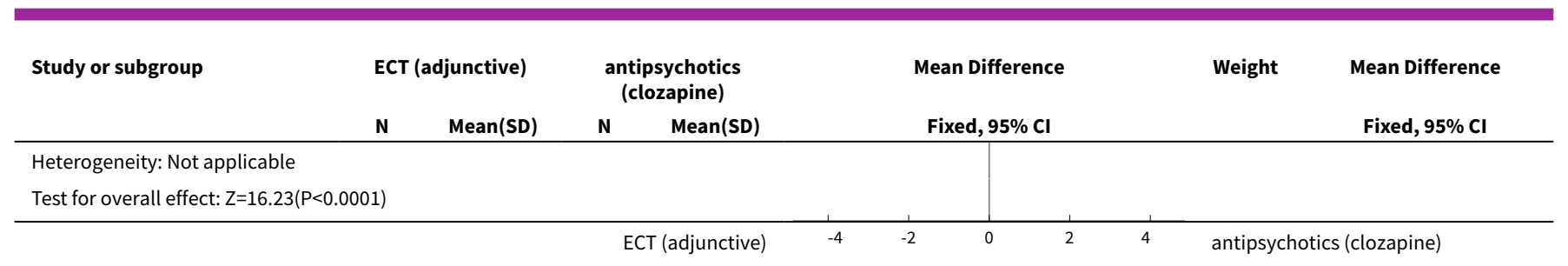

\section{Analysis 2.6. Comparison 2 ECT plus standard care versus antipsychotics plus} standard care, Outcome 6 Adverse events - total score (TESS, high = poor).

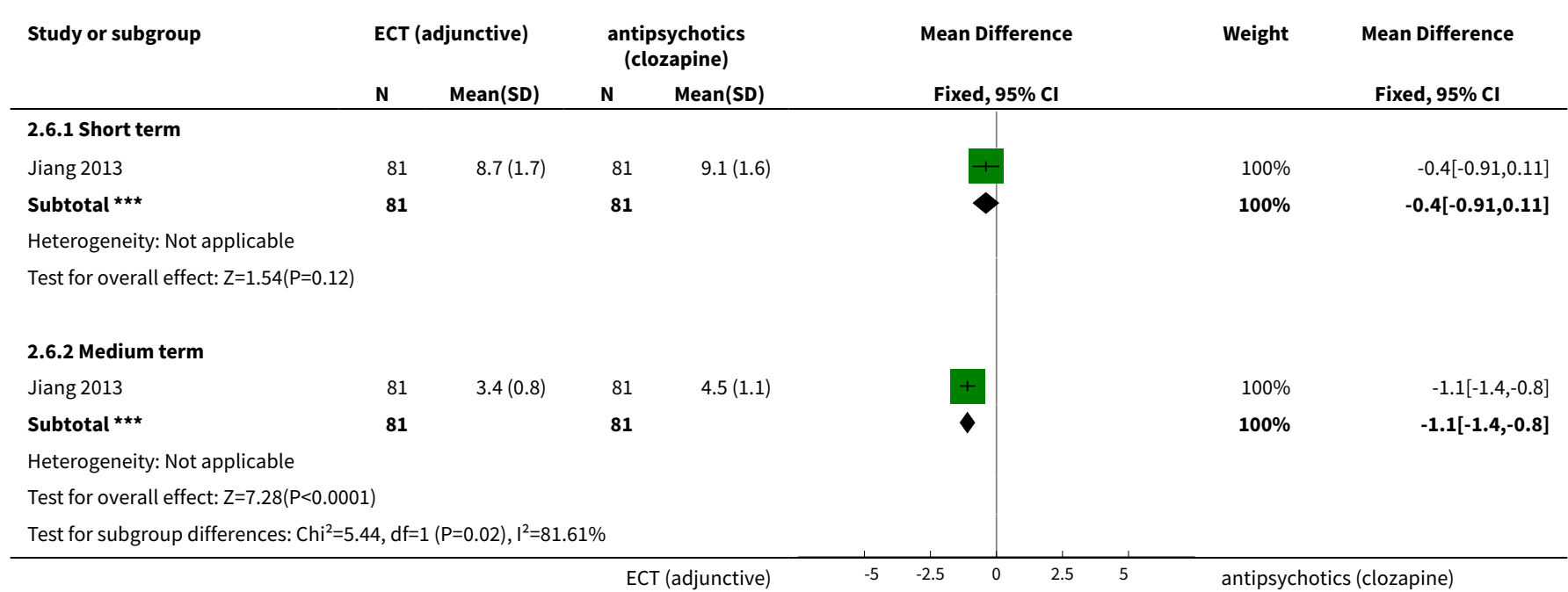

\section{Comparison 3. ECT plus standard care versus standard care}

\begin{tabular}{|c|c|c|c|c|}
\hline $\begin{array}{l}\text { Outcome or subgroup ti- } \\
\text { tle }\end{array}$ & $\begin{array}{l}\text { No. of } \\
\text { studies }\end{array}$ & $\begin{array}{l}\text { No. of } \\
\text { partici- } \\
\text { pants }\end{array}$ & Statistical method & Effect size \\
\hline $\begin{array}{l}1 \text { Response to treatment } \\
\text { - clinically important re- } \\
\text { sponse (as defined by each } \\
\text { study) }\end{array}$ & 10 & & Risk Ratio (M-H, Fixed, 95\% Cl) & Subtotals only \\
\hline 1.1 Short term & 1 & 72 & Risk Ratio (M-H, Fixed, 95\% Cl) & $1.91[1.09,3.36]$ \\
\hline 1.2 Medium term & 9 & 819 & Risk Ratio (M-H, Fixed, 95\% Cl) & $2.06[1.75,2.42]$ \\
\hline $\begin{array}{l}2 \text { Cognitive functioning - } \\
\text { memory deterioration - } \\
\text { short term }\end{array}$ & 1 & 72 & Risk Ratio (M-H, Fixed, 95\% Cl) & $27.0[1.67,437.68]$ \\
\hline $\begin{array}{l}3 \text { Cognitive functioning - } \\
\text { specific symptom score } \\
\text { (WCST, high = poor, oth- } \\
\text { er than correct number) - } \\
\text { medium term }\end{array}$ & 1 & & Mean Difference (IV, Fixed, 95\% CI) & Subtotals only \\
\hline
\end{tabular}




\begin{tabular}{|c|c|c|c|c|}
\hline $\begin{array}{l}\text { Outcome or subgroup ti- } \\
\text { tle }\end{array}$ & $\begin{array}{l}\text { No. of } \\
\text { studies }\end{array}$ & $\begin{array}{l}\text { No. of } \\
\text { partici- } \\
\text { pants }\end{array}$ & Statistical method & Effect size \\
\hline 3.1 Total test number & 1 & 67 & Mean Difference (IV, Fixed, 95\% CI) & $-8.74[-16.88,-0.60]$ \\
\hline 3.2 Correct number & 1 & 67 & Mean Difference (IV, Fixed, 95\% CI) & $3.03[-0.15,6.21]$ \\
\hline 3.3 Categories completed & 1 & 67 & Mean Difference (IV, Fixed, 95\% CI) & $-0.36[-0.49,-0.23]$ \\
\hline $\begin{array}{l}4 \text { Cognitive functioning - } \\
\text { specific symptom score } \\
\text { (WCST, high = poor) - medi- } \\
\text { um term (skewed data) }\end{array}$ & & & Other data & No numeric data \\
\hline 4.1 Perseveration errors & & & Other data & No numeric data \\
\hline $\begin{array}{l}4.2 \text { Non-perseveration er- } \\
\text { rors }\end{array}$ & & & Other data & No numeric data \\
\hline $\begin{array}{l}5 \text { Cognitive functioning } \\
\text { - memory (WMS, high = } \\
\text { good) - short term }\end{array}$ & 1 & & Mean Difference (IV, Fixed, 95\% CI) & Subtotals only \\
\hline 5.1 Memory quotient (MQ) & 1 & 245 & Mean Difference (IV, Fixed, 95\% CI) & $0.27[-3.54,4.08]$ \\
\hline $\begin{array}{l}5.2 \text { Memorise number ( } 1 \text { to } \\
100 \text { ) }\end{array}$ & 1 & 245 & Mean Difference (IV, Fixed, 95\% CI) & $-0.09[-0.71,0.53]$ \\
\hline $\begin{array}{l}5.3 \text { Memorise number ( } 100 \\
\text { to } 1 \text { ) }\end{array}$ & 1 & 245 & Mean Difference (IV, Fixed, 95\% CI) & $-0.30[-0.98,0.38]$ \\
\hline $\begin{array}{l}5.4 \text { Accumulation of mem- } \\
\text { ory }\end{array}$ & 1 & 245 & Mean Difference (IV, Fixed, 95\% CI) & $-0.11[-0.73,0.51]$ \\
\hline 5.5 Picture memory & 1 & 245 & Mean Difference (IV, Fixed, 95\% CI) & $-0.41[-1.01,0.19]$ \\
\hline 5.6 Recognition memory & 1 & 245 & Mean Difference (IV, Fixed, 95\% CI) & $-0.03[-0.64,0.58]$ \\
\hline 5.7 Regeneration memory & 1 & 245 & Mean Difference (IV, Fixed, 95\% CI) & $-0.04[-0.78,0.70]$ \\
\hline 5.8 Associative memory & 1 & 245 & Mean Difference (IV, Fixed, 95\% CI) & $-0.09[-0.97,0.79]$ \\
\hline 5.9 Haptic memory & 1 & 245 & Mean Difference (IV, Fixed, 95\% CI) & $-0.07[-0.53,0.39]$ \\
\hline $\begin{array}{l}5.10 \text { Understanding mem- } \\
\text { ory }\end{array}$ & 1 & 245 & Mean Difference (IV, Fixed, 95\% CI) & $0.02[-0.56,0.60]$ \\
\hline $\begin{array}{l}5.11 \text { Memorise number } \\
\text { (random) }\end{array}$ & 1 & 245 & Mean Difference (IV, Fixed, 95\% CI) & $0.33[-0.29,0.95]$ \\
\hline $\begin{array}{l}6 \text { Cognitive functioning } \\
\text { - memory (WMS, high = } \\
\text { good) - medium term }\end{array}$ & 1 & & Mean Difference (IV, Fixed, 95\% CI) & Subtotals only \\
\hline 6.1 Memory quotient (MQ) & 1 & 245 & Mean Difference (IV, Fixed, 95\% CI) & $1.75[-2.24,5.74]$ \\
\hline
\end{tabular}




\begin{tabular}{|c|c|c|c|c|}
\hline $\begin{array}{l}\text { Outcome or subgroup ti- } \\
\text { tle }\end{array}$ & $\begin{array}{l}\text { No. of } \\
\text { studies }\end{array}$ & $\begin{array}{l}\text { No. of } \\
\text { partici- } \\
\text { pants }\end{array}$ & Statistical method & Effect size \\
\hline $\begin{array}{l}6.2 \text { Memorise number ( } 1 \text { to } \\
100 \text { ) }\end{array}$ & 1 & 245 & Mean Difference (IV, Fixed, 95\% CI) & $-0.06[-0.69,0.57]$ \\
\hline $\begin{array}{l}6.3 \text { Memorise number ( } 100 \\
\text { to } 1 \text { ) }\end{array}$ & 1 & 245 & Mean Difference (IV, Fixed, 95\% CI) & $-0.15[-0.88,0.58]$ \\
\hline $\begin{array}{l}6.4 \text { Accumulation of mem- } \\
\text { ory }\end{array}$ & 1 & 245 & Mean Difference (IV, Fixed, 95\% CI) & $0.03[-0.59,0.65]$ \\
\hline 6.5 Picture memory & 1 & 245 & Mean Difference (IV, Fixed, 95\% CI) & $-0.41[-1.01,0.19]$ \\
\hline 6.6 Recognition memory & 1 & 245 & Mean Difference (IV, Fixed, 95\% CI) & $0.09[-0.52,0.70]$ \\
\hline 6.7 Regeneration memory & 1 & 245 & Mean Difference (IV, Fixed, 95\% CI) & $0.11[-0.64,0.86]$ \\
\hline 6.8 Associative memory & 1 & 245 & Mean Difference (IV, Fixed, 95\% CI) & $0.11[-0.79,1.01]$ \\
\hline 6.9 Haptic memory & 1 & 245 & Mean Difference (IV, Fixed, 95\% CI) & $0.10[-0.38,0.58]$ \\
\hline $\begin{array}{l}6.10 \text { Understanding mem- } \\
\text { ory }\end{array}$ & 1 & 245 & Mean Difference (IV, Fixed, 95\% CI) & $-0.06[-0.63,0.51]$ \\
\hline $\begin{array}{l}\text { 6.11 Memorise number } \\
\text { (random) }\end{array}$ & 1 & 245 & Mean Difference (IV, Fixed, 95\% CI) & $0.24[-0.37,0.85]$ \\
\hline $\begin{array}{l}7 \text { Satisfaction and accept- } \\
\text { ability of treatment - leav- } \\
\text { ing the study early - medi- } \\
\text { um term }\end{array}$ & 3 & 354 & Risk Ratio (M-H, Fixed, 95\% Cl) & $1.18[0.38,3.63]$ \\
\hline $\begin{array}{l}8 \text { Mental state - total score } \\
\text { (BPRS, high = poor) }\end{array}$ & 2 & & Mean Difference (IV, Fixed, 95\% CI) & Subtotals only \\
\hline 8.1 Short term & 2 & 345 & Mean Difference (IV, Fixed, 95\% CI) & $-5.50[-6.99,-4.00]$ \\
\hline 8.2 Medium term & 2 & 345 & Mean Difference (IV, Fixed, 95\% CI) & $-11.18[-12.61,-9.76]$ \\
\hline $\begin{array}{l}9 \text { Mental state - total score } \\
\text { (BPRS, high = poor) - medi- } \\
\text { um term (skewed data) }\end{array}$ & & & Other data & No numeric data \\
\hline $\begin{array}{l}10 \text { Mental state - total } \\
\text { score (MMSE, high = good) } \\
\text { - medium term }\end{array}$ & 2 & 69 & Mean Difference (IV, Fixed, 95\% CI) & $0.98[0.30,1.65]$ \\
\hline $\begin{array}{l}11 \text { Mental state - total } \\
\text { score (PANSS, high = poor) }\end{array}$ & 6 & & Mean Difference (IV, Fixed, 95\% CI) & Subtotals only \\
\hline 11.1 Short term & 6 & 432 & Mean Difference (IV, Fixed, 95\% CI) & $-11.41[-13.49,-9.34]$ \\
\hline 11.2 Medium term & 6 & 432 & Mean Difference (IV, Fixed, 95\% CI) & $-7.62[-9.49,-5.74]$ \\
\hline
\end{tabular}




\begin{tabular}{|c|c|c|c|c|}
\hline $\begin{array}{l}\text { Outcome or subgroup ti- } \\
\text { tle }\end{array}$ & $\begin{array}{l}\text { No. of } \\
\text { studies }\end{array}$ & $\begin{array}{l}\text { No. of } \\
\text { partici- } \\
\text { pants }\end{array}$ & Statistical method & Effect size \\
\hline $\begin{array}{l}12 \text { Mental state - specif- } \\
\text { ic symptom score (BPRS, } \\
\text { high = poor) - short term }\end{array}$ & 3 & & Mean Difference (IV, Fixed, 95\% CI) & Subtotals only \\
\hline $\begin{array}{l}12.1 \text { Anxiety and depres- } \\
\text { sion }\end{array}$ & 2 & 345 & Mean Difference (IV, Fixed, 95\% CI) & $-0.97[-1.28,-0.65]$ \\
\hline 12.2 Lack of vitality & 2 & 345 & Mean Difference (IV, Fixed, 95\% CI) & $-2.40[-2.71,-2.09]$ \\
\hline 12.3 Thought disturbance & 2 & 345 & Mean Difference (IV, Fixed, 95\% CI) & $-0.71[-1.06,-0.35]$ \\
\hline 12.4 Agitation & 2 & 345 & Mean Difference (IV, Fixed, 95\% CI) & $-1.23[-1.51,-0.95]$ \\
\hline $\begin{array}{l}12.5 \text { Hostile suspicious- } \\
\text { ness }\end{array}$ & 2 & 345 & Mean Difference (IV, Fixed, 95\% CI) & $-0.57[-0.91,-0.24]$ \\
\hline 12.6 Psychosis & 1 & 39 & Mean Difference (IV, Fixed, 95\% CI) & $-5.24[-5.89,-4.59]$ \\
\hline $\begin{array}{l}13 \text { Mental state - specif- } \\
\text { ic symptom score (BPRS, } \\
\text { high = poor) - medium } \\
\text { term }\end{array}$ & 4 & & Mean Difference (IV, Fixed, 95\% CI) & Subtotals only \\
\hline $\begin{array}{l}13.1 \text { Anxiety and depres- } \\
\text { sion }\end{array}$ & 3 & 423 & Mean Difference (IV, Fixed, 95\% CI) & $-1.50[-1.72,-1.29]$ \\
\hline 13.2 Lack of vitality & 3 & 423 & Mean Difference (IV, Fixed, 95\% CI) & $-1.80[-2.02,-1.58]$ \\
\hline 13.3 Thought disturbance & 3 & 423 & Mean Difference (IV, Fixed, 95\% CI) & $-2.69[-2.95,-2.43]$ \\
\hline 13.4 Agitation & 3 & 423 & Mean Difference (IV, Fixed, 95\% CI) & $-1.73[-1.91,-1.54]$ \\
\hline $\begin{array}{l}13.5 \text { Hostile suspicious- } \\
\text { ness }\end{array}$ & 3 & 423 & Mean Difference (IV, Fixed, 95\% CI) & $-2.77[-3.00,-2.54]$ \\
\hline 13.6 Psychosis & 1 & 39 & Mean Difference (IV, Fixed, 95\% CI) & $-6.07[-6.72,-5.42]$ \\
\hline $\begin{array}{l}14 \text { Mental state - specific } \\
\text { symptom score (PANSS, } \\
\text { high = poor) - short term }\end{array}$ & 6 & & Mean Difference (IV, Fixed, 95\% CI) & Subtotals only \\
\hline 14.1 Positive symptom & 3 & 231 & Mean Difference (IV, Fixed, 95\% CI) & $-1.56[-3.12,0.00]$ \\
\hline 14.2 Negative symptom & 5 & 372 & Mean Difference (IV, Fixed, 95\% CI) & $-1.03[-2.42,0.35]$ \\
\hline $\begin{array}{l}14.3 \text { General psy- } \\
\text { chopathology }\end{array}$ & 3 & 234 & Mean Difference (IV, Fixed, 95\% CI) & $-1.59[-3.83,0.66]$ \\
\hline 14.4 Excitement & 1 & 60 & Mean Difference (IV, Fixed, 95\% CI) & $-5.79[-7.34,-4.24]$ \\
\hline 14.5 Hostility & 1 & 60 & Mean Difference (IV, Fixed, 95\% CI) & $-2.48[-3.93,-1.03]$ \\
\hline $\begin{array}{l}15 \text { Mental state - specific } \\
\text { symptom score (PANSS, }\end{array}$ & & & Other data & No numeric data \\
\hline
\end{tabular}




\begin{tabular}{|c|c|c|c|c|}
\hline $\begin{array}{l}\text { Outcome or subgroup ti- } \\
\text { tle }\end{array}$ & $\begin{array}{l}\text { No. of } \\
\text { studies }\end{array}$ & $\begin{array}{l}\text { No. of } \\
\text { partici- } \\
\text { pants }\end{array}$ & Statistical method & Effect size \\
\hline \multicolumn{5}{|l|}{$\begin{array}{l}\text { high = poor) - short term } \\
\text { (skewed data) }\end{array}$} \\
\hline 15.1 Positive symptom & & & Other data & No numeric data \\
\hline $\begin{array}{l}15.2 \text { General psy- } \\
\text { chopathology }\end{array}$ & & & Other data & No numeric data \\
\hline $\begin{array}{l}16 \text { Mental state - specific } \\
\text { symptom score (PANSS, } \\
\text { high = poor) - medium } \\
\text { term }\end{array}$ & 5 & & Mean Difference (IV, Fixed, 95\% CI) & Subtotals only \\
\hline 16.1 Negative symptom & 3 & 229 & Mean Difference (IV, Fixed, 95\% CI) & $0.58[-1.47,2.62]$ \\
\hline $\begin{array}{l}16.2 \text { General psy- } \\
\text { chopathology }\end{array}$ & 2 & 150 & Mean Difference (IV, Fixed, 95\% CI) & $-2.93[-5.32,-0.54]$ \\
\hline 16.3 Excitement & 1 & 60 & Mean Difference (IV, Fixed, 95\% CI) & $-2.31[-3.44,-1.18]$ \\
\hline 16.4 Hostility & 1 & 60 & Mean Difference (IV, Fixed, 95\% CI) & $-2.81[-4.05,-1.57]$ \\
\hline $\begin{array}{l}17 \text { Mental state - specific } \\
\text { symptom score (PANSS, } \\
\text { high = poor) - medium } \\
\text { term (skewed data) }\end{array}$ & & & Other data & No numeric data \\
\hline 17.1 Positive symptom & & & Other data & No numeric data \\
\hline 17.2 Negative symptom & & & Other data & No numeric data \\
\hline $\begin{array}{l}17.3 \text { General psy- } \\
\text { chopathology }\end{array}$ & & & Other data & No numeric data \\
\hline $\begin{array}{l}18 \text { Mental state - specif- } \\
\text { ic symptom score (SAPS, } \\
\text { high = poor) - short term }\end{array}$ & 1 & & Mean Difference (IV, Fixed, 95\% CI) & Subtotals only \\
\hline 18.1 Hallucinations & 1 & 72 & Mean Difference (IV, Fixed, 95\% CI) & $-1.5[-1.71,-1.29]$ \\
\hline 18.2 Delusions & 1 & 72 & Mean Difference (IV, Fixed, 95\% CI) & $-2.81[-3.10,-2.52]$ \\
\hline 18.3 Bizarre behaviour & 1 & 72 & Mean Difference (IV, Fixed, 95\% CI) & $-0.10[-0.21,0.01]$ \\
\hline $\begin{array}{l}\text { 18.4 Positive formal } \\
\text { thought disorder }\end{array}$ & 1 & 72 & Mean Difference (IV, Fixed, 95\% CI) & $-2.2[-2.50,-1.90]$ \\
\hline $\begin{array}{l}19 \text { Mental state - specif- } \\
\text { ic symptom score (SANS, } \\
\text { high = poor) - short term }\end{array}$ & 1 & & Mean Difference (IV, Fixed, 95\% CI) & Subtotals only \\
\hline $\begin{array}{l}19.1 \text { Affective flattening or } \\
\text { blunting }\end{array}$ & 1 & 72 & Mean Difference (IV, Fixed, 95\% CI) & $-4.70[-5.03,-4.37]$ \\
\hline
\end{tabular}




\begin{tabular}{|c|c|c|c|c|}
\hline $\begin{array}{l}\text { Outcome or subgroup ti- } \\
\text { tle }\end{array}$ & $\begin{array}{l}\text { No. of } \\
\text { studies }\end{array}$ & $\begin{array}{l}\text { No. of } \\
\text { partici- } \\
\text { pants }\end{array}$ & Statistical method & Effect size \\
\hline 19.2 Alogia & 1 & 72 & Mean Difference (IV, Fixed, 95\% CI) & $-0.20[-0.43,0.03]$ \\
\hline 19.3 Avolition & 1 & 72 & Mean Difference (IV, Fixed, 95\% CI) & $-0.20[-0.40,0.00]$ \\
\hline 19.4 Anhedonia & 1 & 72 & Mean Difference (IV, Fixed, 95\% CI) & $-0.30[-0.44,-0.16]$ \\
\hline 19.5 Attention & 1 & 72 & Mean Difference (IV, Fixed, 95\% CI) & $-0.90[-0.97,-0.83]$ \\
\hline $\begin{array}{l}20 \text { General functioning - } \\
\text { total score (GAF, high = } \\
\text { good) }\end{array}$ & 2 & & Mean Difference (IV, Fixed, 95\% CI) & Subtotals only \\
\hline 20.1 Short term & 1 & 67 & Mean Difference (IV, Fixed, 95\% CI) & $4.32[-0.20,8.84]$ \\
\hline 20.2 Medium term & 2 & 97 & Mean Difference (IV, Fixed, 95\% CI) & $10.66[6.98,14.34]$ \\
\hline $\begin{array}{l}21 \text { Adverse events - gener- } \\
\text { al - medium term }\end{array}$ & 1 & 84 & Risk Ratio (M-H, Fixed, 95\% Cl) & $1.33[0.86,2.06]$ \\
\hline $\begin{array}{l}22 \text { Adverse events - total } \\
\text { score (TESS, high = poor) }\end{array}$ & 4 & & Mean Difference (IV, Fixed, 95\% CI) & Subtotals only \\
\hline 22.1 Short term & 3 & 251 & Mean Difference (IV, Fixed, 95\% CI) & $-0.19[-0.96,0.57]$ \\
\hline 22.2 Medium term & 4 & 496 & Mean Difference (IV, Fixed, 95\% CI) & $-0.63[-1.01,-0.25]$ \\
\hline $\begin{array}{l}23 \text { Adverse events - specif- } \\
\text { ic symptom - short term }\end{array}$ & 1 & & Risk Ratio (M-H, Fixed, 95\% Cl) & Subtotals only \\
\hline $\begin{array}{l}23.1 \text { Abnormal electrocar- } \\
\text { diogram }\end{array}$ & 1 & 72 & Risk Ratio (M-H, Fixed, 95\% Cl) & $0.85[0.44,1.63]$ \\
\hline $\begin{array}{l}\text { 23.2 Abnormal liver func- } \\
\text { tion }\end{array}$ & 1 & 72 & Risk Ratio (M-H, Fixed, 95\% Cl) & $0.67[0.12,3.75]$ \\
\hline 23.3 Constipation & 1 & 72 & Risk Ratio (M-H, Fixed, 95\% Cl) & $0.75[0.36,1.56]$ \\
\hline 23.4 Headache & 1 & 72 & Risk Ratio (M-H, Fixed, 95\% Cl) & $7.0[0.37,130.82]$ \\
\hline 23.5 Lethargy & 1 & 72 & Risk Ratio (M-H, Fixed, 95\% Cl) & $0.8[0.44,1.46]$ \\
\hline 23.6 Nausea or vomiting & 1 & 72 & Risk Ratio (M-H, Fixed, 95\% Cl) & $2.0[0.19,21.09]$ \\
\hline 23.7 Salivation & 1 & 72 & Risk Ratio (M-H, Fixed, 95\% Cl) & $0.73[0.33,1.59]$ \\
\hline 23.8 Weight gain & 1 & 72 & Risk Ratio (M-H, Fixed, 95\% Cl) & $0.4[0.14,1.16]$ \\
\hline $\begin{array}{l}24 \text { Adverse events - spe- } \\
\text { cific symptom - medium } \\
\text { term }\end{array}$ & 6 & & Risk Ratio (M-H, Fixed, 95\% Cl) & Subtotals only \\
\hline $\begin{array}{l}24.1 \text { Abnormal electrocar- } \\
\text { diogram }\end{array}$ & 4 & 274 & Risk Ratio (M-H, Fixed, 95\% Cl) & $0.92[0.67,1.26]$ \\
\hline
\end{tabular}




\begin{tabular}{|c|c|c|c|c|}
\hline $\begin{array}{l}\text { Outcome or subgroup ti- } \\
\text { tle }\end{array}$ & $\begin{array}{l}\text { No. of } \\
\text { studies }\end{array}$ & $\begin{array}{l}\text { No. of } \\
\text { partici- } \\
\text { pants }\end{array}$ & Statistical method & Effect size \\
\hline $\begin{array}{l}24.2 \text { Abnormal electroen- } \\
\text { cephalogram }\end{array}$ & 1 & 60 & Risk Ratio (M-H, Fixed, 95\% Cl) & $1.04[0.82,1.32]$ \\
\hline 24.4 Blurred vision & 1 & 69 & Risk Ratio (M-H, Fixed, 95\% Cl) & $1.03[0.28,3.79]$ \\
\hline 24.5 Breathing discomfort & 1 & 78 & Risk Ratio (M-H, Fixed, 95\% Cl) & $0.08[0.01,0.61]$ \\
\hline 24.7 Constipation & 2 & 145 & Risk Ratio (M-H, Fixed, 95\% Cl) & $0.76[0.61,0.95]$ \\
\hline 24.8 Decreased activity & 1 & 69 & Risk Ratio (M-H, Fixed, 95\% Cl) & $0.56[0.23,1.35]$ \\
\hline 24.9 Dizziness & 1 & 69 & Risk Ratio (M-H, Fixed, 95\% Cl) & $1.72[0.70,4.20]$ \\
\hline 24.10 Dry mouth & 1 & 69 & Risk Ratio (M-H, Fixed, 95\% Cl) & $1.03[0.33,3.24]$ \\
\hline 24.11 Headache & 2 & 147 & Risk Ratio (M-H, Fixed, 95\% Cl) & $5.47[1.47,20.39]$ \\
\hline 24.12 Indigestion & 1 & 78 & Risk Ratio (M-H, Fixed, 95\% Cl) & $0.5[0.16,1.53]$ \\
\hline 24.13 Insomnia & 1 & 69 & Risk Ratio (M-H, Fixed, 95\% Cl) & $0.05[0.00,0.80]$ \\
\hline 24.15 Leucopenia & 2 & 145 & Risk Ratio (M-H, Fixed, 95\% Cl) & $0.73[0.18,3.03]$ \\
\hline 24.16 Muscle rigidity & 1 & 69 & Risk Ratio (M-H, Fixed, 95\% Cl) & $0.77[0.37,1.59]$ \\
\hline 24.17 Nausea or vomiting & 2 & 147 & Risk Ratio (M-H, Fixed, 95\% Cl) & $0.79[0.31,2.02]$ \\
\hline $\begin{array}{l}24.18 \text { Recurrence of pre- } \\
\text { existing involuntary 'jerky' } \\
\text { movements }\end{array}$ & 1 & 39 & Risk Ratio (M-H, Fixed, 95\% Cl) & $2.86[0.12,66.11]$ \\
\hline 24.19 Salivation & 2 & 145 & Risk Ratio (M-H, Fixed, 95\% Cl) & $0.80[0.57,1.14]$ \\
\hline 24.20 Tremor & 1 & 69 & Risk Ratio (M-H, Fixed, 95\% Cl) & $0.72[0.31,1.67]$ \\
\hline 24.21 Weight gain & 3 & 214 & Risk Ratio (M-H, Fixed, 95\% Cl) & $0.72[0.46,1.13]$ \\
\hline 24.22 Others & 2 & 145 & Risk Ratio (M-H, Fixed, 95\% Cl) & $0.99[0.44,2.23]$ \\
\hline $\begin{array}{l}25 \text { Adverse events - specif- } \\
\text { ic symptom score (TESS, } \\
\text { high = poor) - short term } \\
\text { (skewed data) }\end{array}$ & & & Other data & No numeric data \\
\hline
\end{tabular}




\begin{tabular}{|c|c|c|c|c|}
\hline $\begin{array}{l}\text { Outcome or subgroup ti- } \\
\text { tle }\end{array}$ & $\begin{array}{l}\text { No. of } \\
\text { studies }\end{array}$ & $\begin{array}{l}\text { No. of } \\
\text { partici- } \\
\text { pants }\end{array}$ & Statistical method & Effect size \\
\hline 25.2 Behavioural toxicity & & & Other data & No numeric data \\
\hline $\begin{array}{l}25.3 \text { Symptoms of cardio- } \\
\text { vascular system }\end{array}$ & & & Other data & No numeric data \\
\hline $\begin{array}{l}25.4 \text { Symptoms of nervous } \\
\text { system }\end{array}$ & & & Other data & No numeric data \\
\hline $\begin{array}{l}25.5 \text { Symptoms of vegeta- } \\
\text { tive nervous system }\end{array}$ & & & Other data & No numeric data \\
\hline 25.6 Others & & & Other data & No numeric data \\
\hline $\begin{array}{l}26 \text { Adverse events - specif- } \\
\text { ic symptom score (TESS, } \\
\text { high = poor) - medium } \\
\text { term }\end{array}$ & 1 & & Mean Difference (IV, Fixed, 95\% CI) & Subtotals only \\
\hline $\begin{array}{l}26.1 \text { Abnormal laboratory } \\
\text { values }\end{array}$ & 1 & 245 & Mean Difference (IV, Fixed, 95\% CI) & $-0.01[-0.08,0.06]$ \\
\hline 26.2 Behavioural toxicity & 1 & 245 & Mean Difference (IV, Fixed, 95\% CI) & $-0.31[-0.38,-0.24]$ \\
\hline $\begin{array}{l}\text { 26.3 Symptoms of cardio- } \\
\text { vascular system }\end{array}$ & 1 & 245 & Mean Difference (IV, Fixed, 95\% CI) & $-0.5[-0.58,-0.42]$ \\
\hline $\begin{array}{l}26.4 \text { Symptoms of nervous } \\
\text { system }\end{array}$ & 1 & 245 & Mean Difference (IV, Fixed, 95\% CI) & $-0.02[-0.08,0.04]$ \\
\hline $\begin{array}{l}26.5 \text { Symptoms of vegeta- } \\
\text { tive nervous system }\end{array}$ & 1 & 245 & Mean Difference (IV, Fixed, 95\% CI) & $0.03[-0.05,0.11]$ \\
\hline 26.6 Others & 1 & 245 & Mean Difference (IV, Fixed, 95\% CI) & $-0.02[-0.17,0.13]$ \\
\hline
\end{tabular}

\begin{tabular}{|c|c|c|}
\hline $\begin{array}{l}27 \text { Adverse events - specif- } \\
\text { ic symptom score (TESS, } \\
\text { high = poor) - medium } \\
\text { term (skewed data) }\end{array}$ & Other data & No numeric data \\
\hline
\end{tabular}

\begin{tabular}{llc}
\hline $\begin{array}{l}27.1 \text { Abnormal laboratory } \\
\text { values }\end{array}$ & Other data & No numeric data \\
\hline 27.2 Behavioral toxicity & Other data & No numeric data \\
\hline $\begin{array}{l}27.3 \text { Symptoms of cardio- } \\
\text { vascular system }\end{array}$ & Other data & No numeric data \\
\hline $\begin{array}{l}27.4 \text { Symptoms of nervous } \\
\text { system }\end{array}$ & Other data & No numeric data \\
\hline $\begin{array}{l}27.5 \text { Symptoms of vegeta- } \\
\text { tive nervous system }\end{array}$ & Other data & No numeric data \\
\hline 27.6 Others & Other data & No numeric data \\
\hline
\end{tabular}


Analysis 3.1. Comparison 3 ECT plus standard care versus standard care, Outcome 1 Response to treatment - clinically important response (as defined by each study).

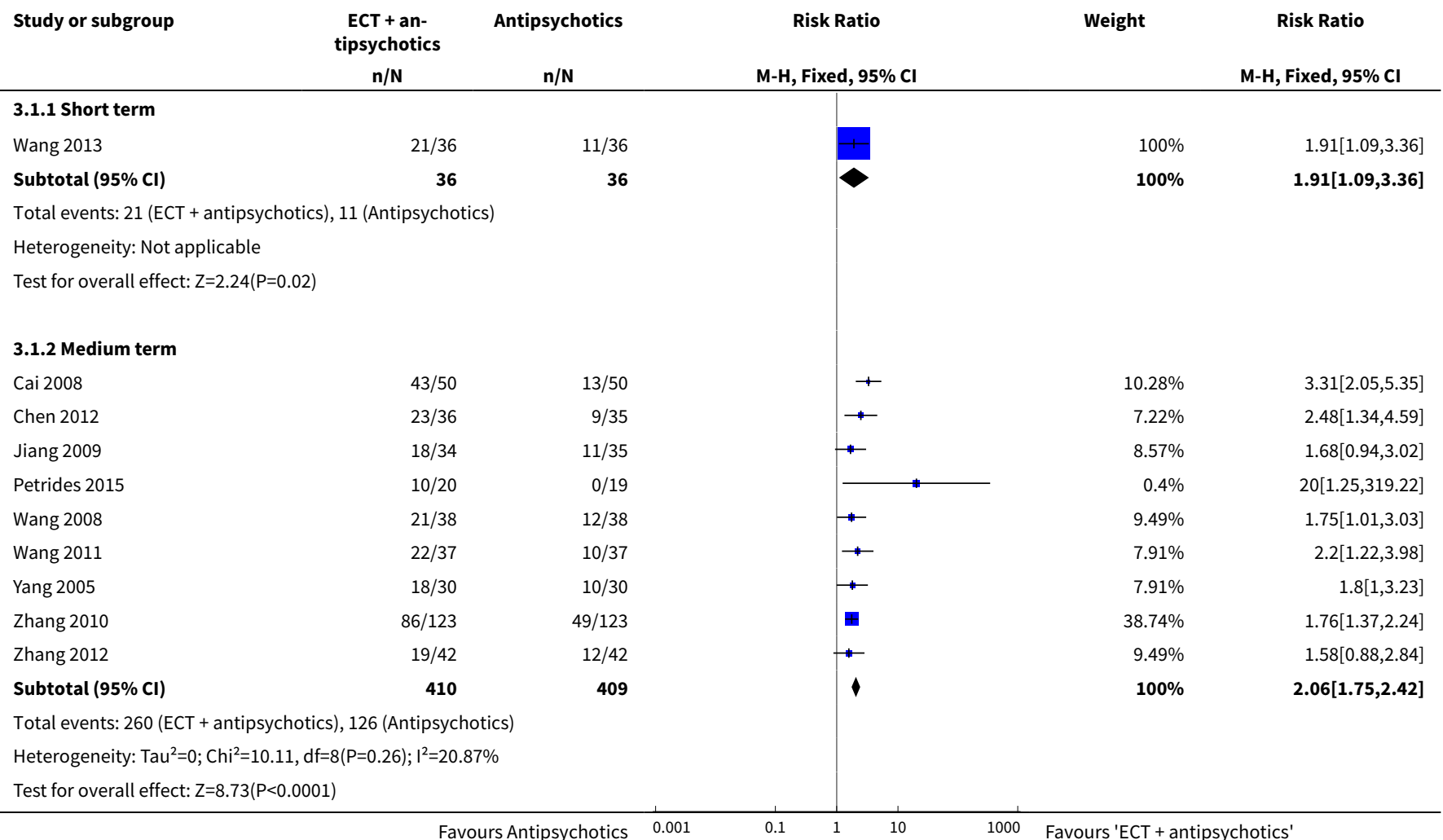

Analysis 3.2. Comparison 3 ECT plus standard care versus standard care, Outcome 2 Cognitive functioning - memory deterioration - short term.

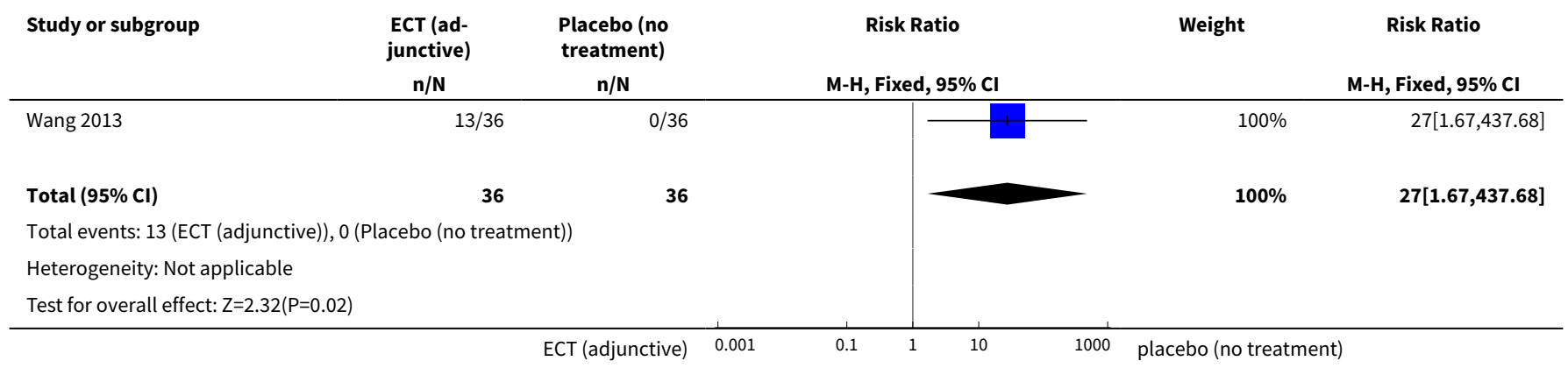


Analysis 3.3. Comparison 3 ECT plus standard care versus standard care, Outcome 3 Cognitive functioning - specific symptom score (WCST, high = poor, other than correct number) - medium term.

\begin{tabular}{|c|c|c|c|c|c|c|c|}
\hline \multirow[t]{2}{*}{ Study or subgroup } & \multicolumn{2}{|c|}{ ECT (adjunctive) } & \multicolumn{2}{|c|}{$\begin{array}{l}\text { Placebo (no } \\
\text { treatment) }\end{array}$} & \multirow{2}{*}{$\begin{array}{c}\text { Mean Difference } \\
\text { Fixed, } 95 \% \mathrm{Cl}\end{array}$} & \multirow[t]{2}{*}{ Weight } & \multirow{2}{*}{$\begin{array}{l}\text { Mean Difference } \\
\text { Fixed, } 95 \% \mathrm{Cl}\end{array}$} \\
\hline & $\mathbf{N}$ & Mean(SD) & $\mathbf{N}$ & Mean(SD) & & & \\
\hline \multicolumn{8}{|c|}{ 3.3.1 Total test number } \\
\hline Jiang 2009 & 32 & $75.4(19.2)$ & 35 & $84.2(14.2)$ & +12 & $100 \%$ & $-8.74[-16.88,-0.6]$ \\
\hline Subtotal $\star \star \star$ & 32 & & 35 & & & $100 \%$ & $-8.74[-16.88,-0.6]$ \\
\hline \multicolumn{8}{|c|}{ Heterogeneity: Not applicable } \\
\hline \multicolumn{8}{|c|}{ 3.3.2 Correct number } \\
\hline Jiang 2009 & 32 & $34.5(5.2)$ & 35 & $31.5(7.9)$ & & $100 \%$ & $3.03[-0.15,6.21]$ \\
\hline Subtotal $\star \star \star$ & 32 & & 35 & & & $100 \%$ & $3.03[-0.15,6.21]$ \\
\hline \multicolumn{8}{|c|}{ Heterogeneity: $\operatorname{Tau}^{2}=0 ; \mathrm{Chi}^{2}=0, \mathrm{df}=0(\mathrm{P}<0.0001) ; \mathrm{I}^{2}=100 \%$} \\
\hline \multicolumn{8}{|c|}{ Test for overall effect: $Z=1.87(P=0.06)$} \\
\hline \multicolumn{8}{|c|}{ 3.3.3 Categories completed } \\
\hline Jiang 2009 & 32 & $3.8(0.3)$ & 35 & $4.1(0.3)$ & & $100 \%$ & $-0.36[-0.49,-0.23]$ \\
\hline Subtotal $* \star \star$ & 32 & & 35 & & 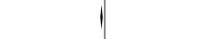 & $100 \%$ & $-0.36[-0.49,-0.23]$ \\
\hline \multicolumn{8}{|c|}{ Heterogeneity: Not applicable } \\
\hline \multicolumn{8}{|c|}{ Test for overall effect: $Z=5.26(P<0.0001)$} \\
\hline
\end{tabular}

Analysis 3.4. Comparison 3 ECT plus standard care versus standard care, Outcome 4 Cognitive functioning - specific symptom score (WCST, high = poor) - medium term (skewed data).

\begin{tabular}{|c|c|c|c|c|}
\hline \multicolumn{5}{|c|}{ Cognitive functioning - specific symptom score (WCST, high = poor) - medium term (skewed data) } \\
\hline Study & Intervention & Mean & SD & $\mathbf{N}$ \\
\hline \multicolumn{5}{|c|}{ Perseveration errors } \\
\hline Jiang 2009 & $\mathrm{MECT}+$ risperidone & 20.12 & 12.47 & 32 \\
\hline Jiang 2009 & Risperidone alone & 27.94 & 14.16 & 35 \\
\hline \multicolumn{5}{|c|}{ Non-perseveration errors } \\
\hline Jiang 2009 & $\mathrm{MECT}+$ risperidone & 25.13 & 15.32 & 32 \\
\hline
\end{tabular}

Analysis 3.5. Comparison 3 ECT plus standard care versus standard care, Outcome 5 Cognitive functioning - memory (WMS, high = good) - short term.

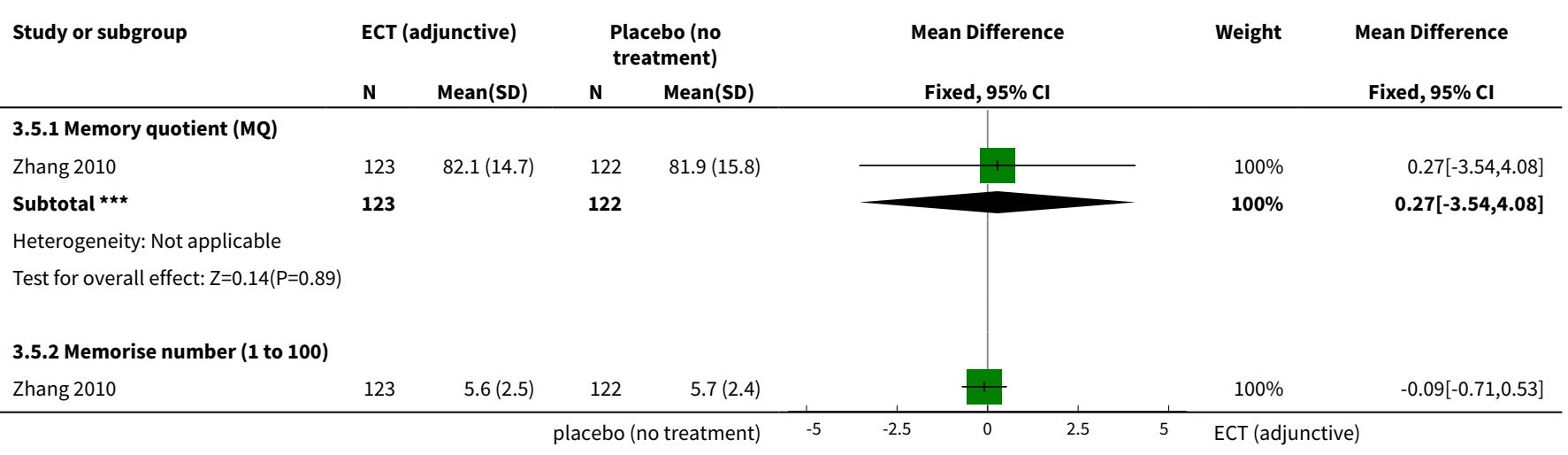




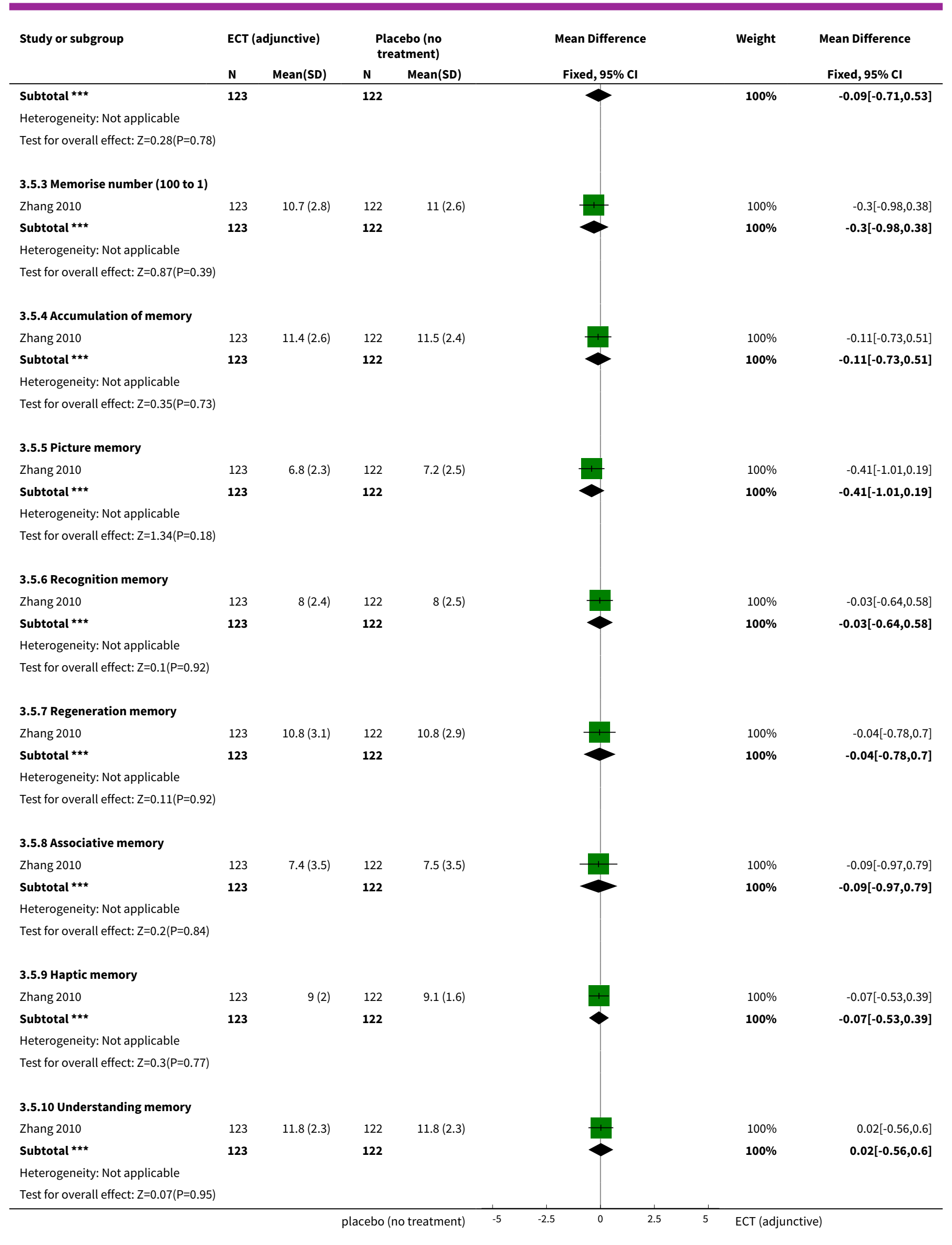




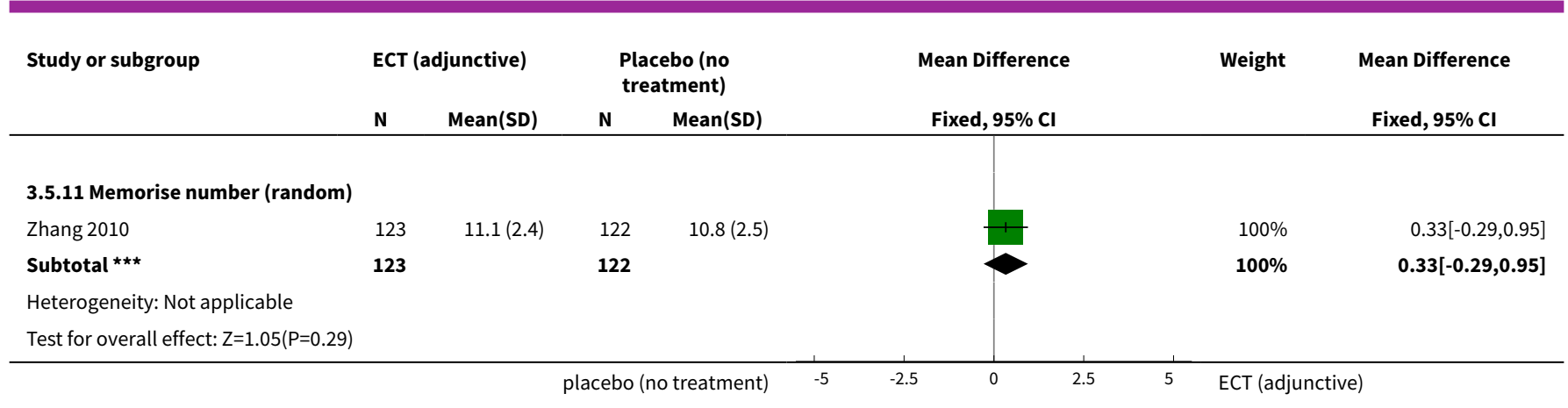

Analysis 3.6. Comparison 3 ECT plus standard care versus standard care, Outcome 6 Cognitive functioning - memory (WMS, high = good) - medium term.

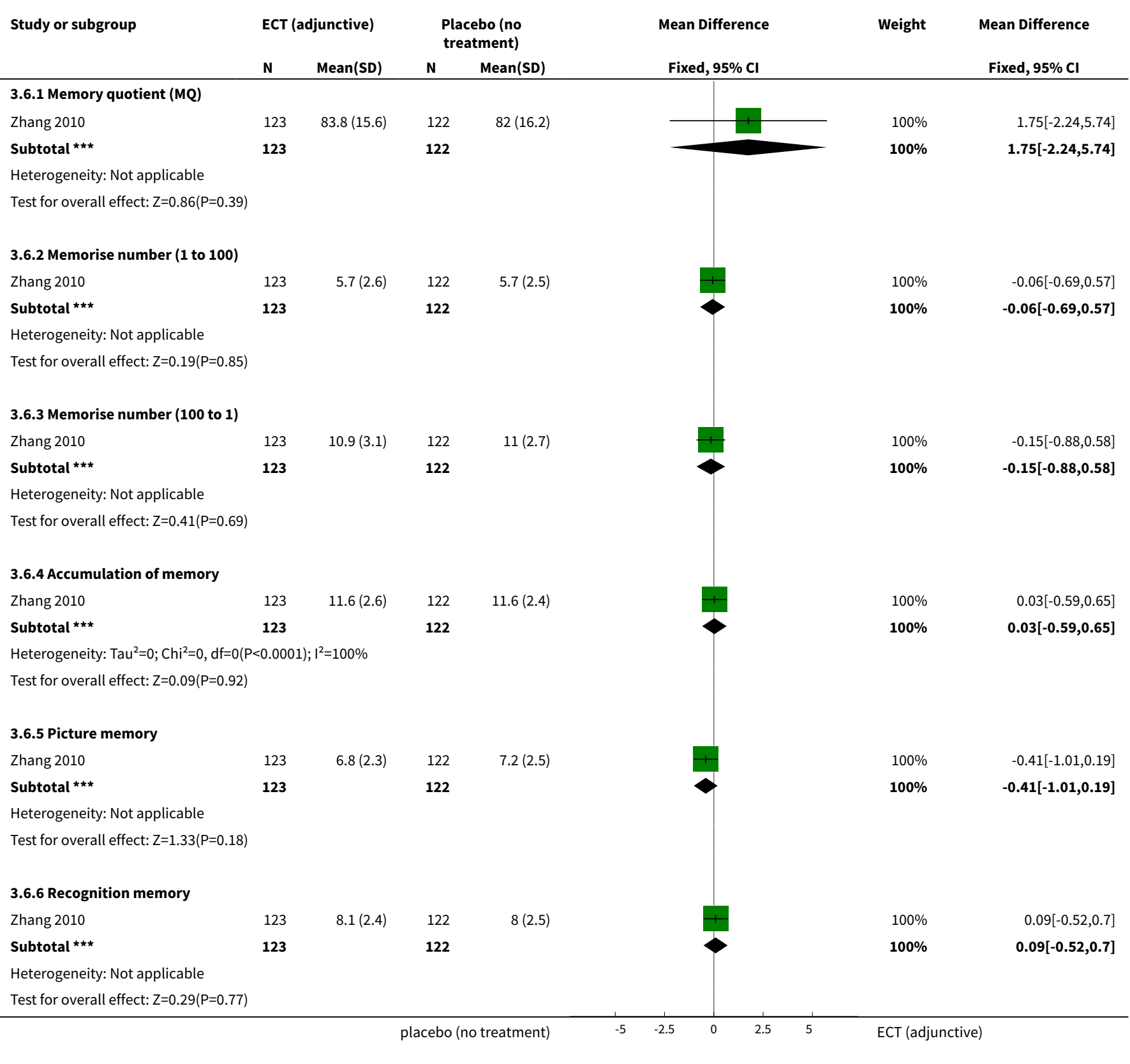




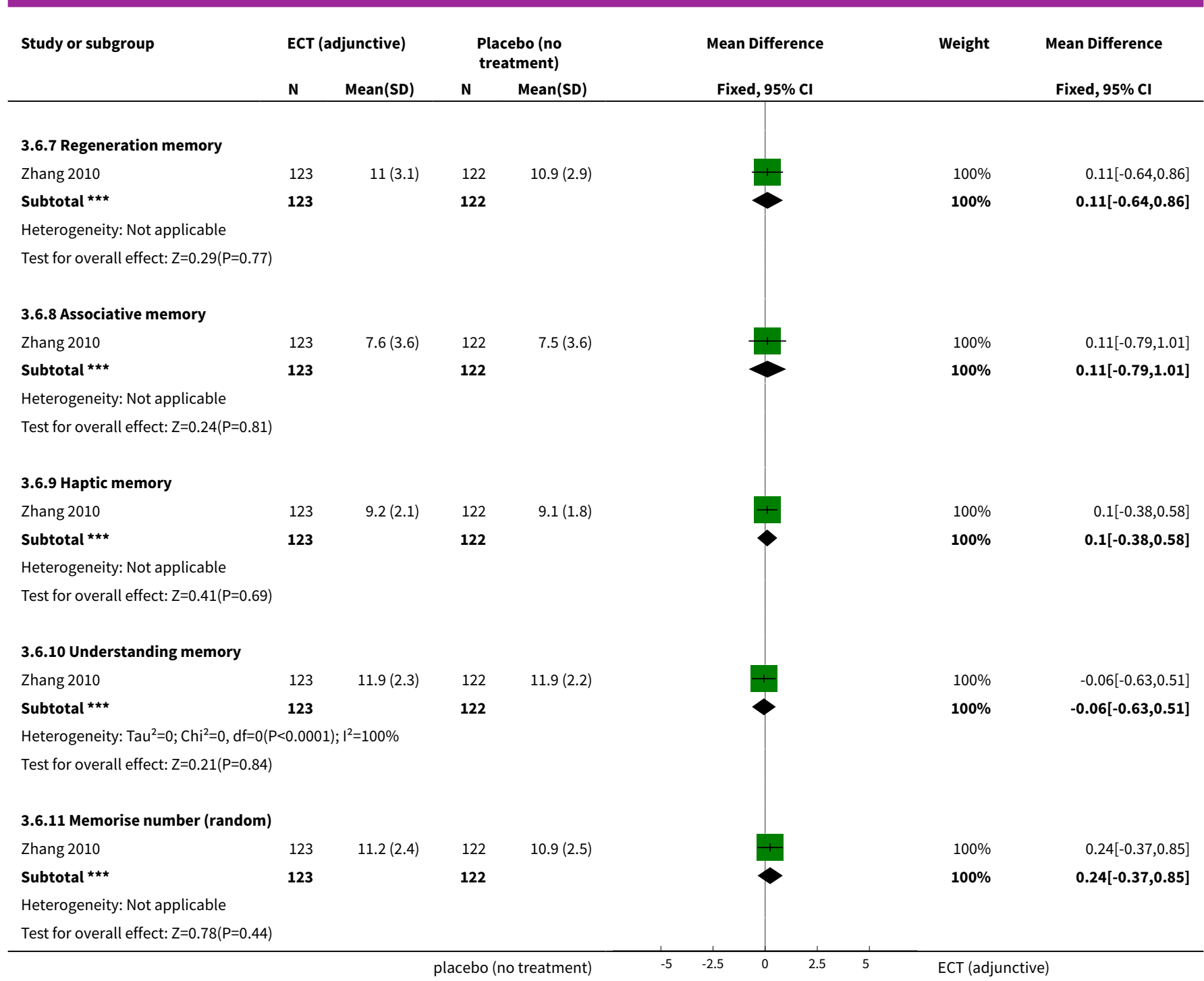

Analysis 3.7. Comparison 3 ECT plus standard care versus standard care, Outcome 7 Satisfaction and acceptability of treatment - leaving the study early - medium term.

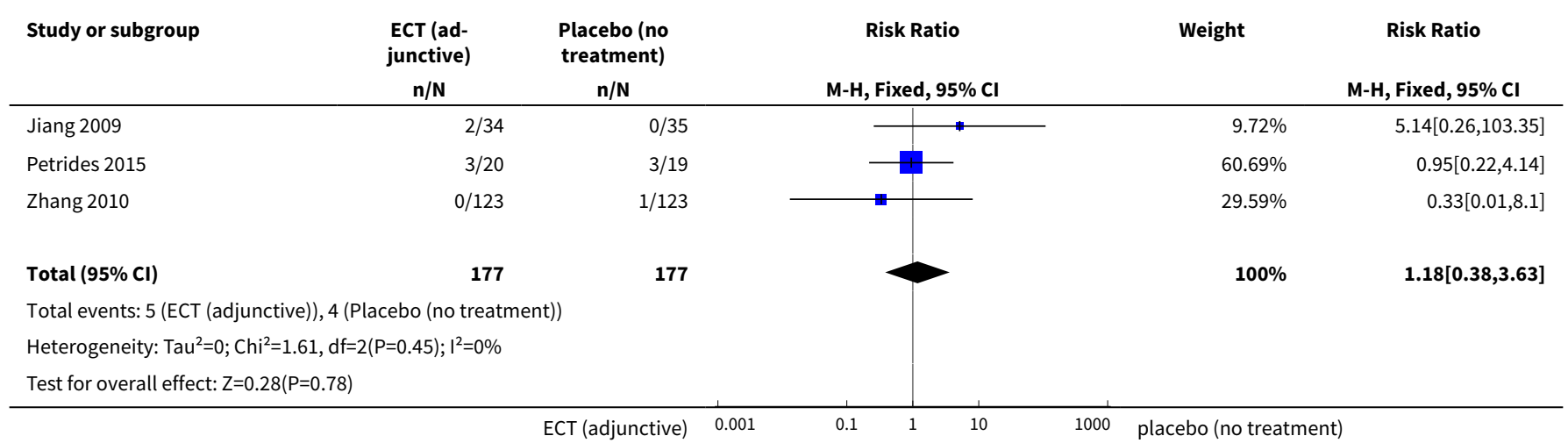




\section{Analysis 3.8. Comparison 3 ECT plus standard care versus standard} care, Outcome 8 Mental state - total score (BPRS, high = poor).

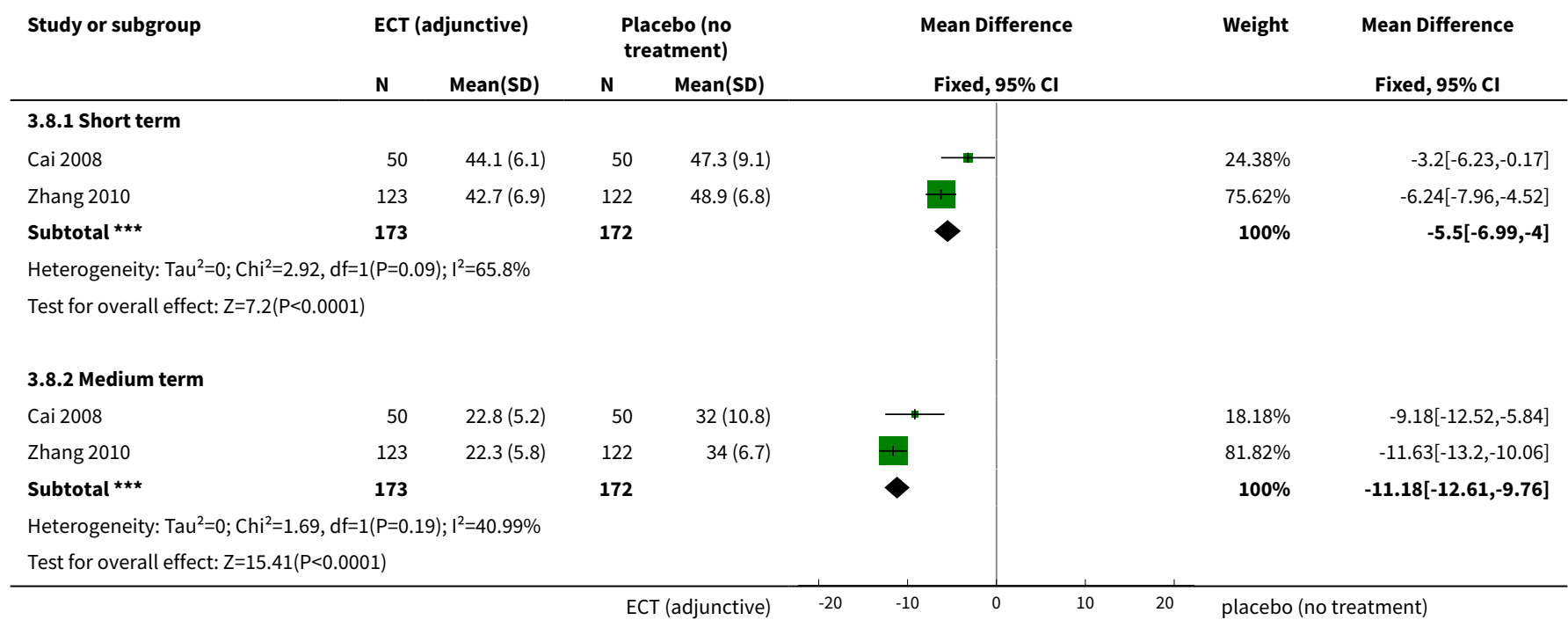

Analysis 3.9. Comparison 3 ECT plus standard care versus standard care, Outcome 9 Mental state - total score (BPRS, high = poor) - medium term (skewed data).

Mental state - total score (BPRS, high = poor) - medium term (skewed data)

\begin{tabular}{rllll} 
Study & \multicolumn{1}{c}{ Interventions } & Mean & SD & N \\
\hline Chanpattana 1999 & ECT + flupenthixol & 24.27 & 16.77 & \\
\hline Chanpattana 1999 & Flupenthixol alone & 44.33 & 8.43 & \\
\hline
\end{tabular}

Analysis 3.10. Comparison 3 ECT plus standard care versus standard care, Outcome 10 Mental state - total score (MMSE, high = good) - medium term.

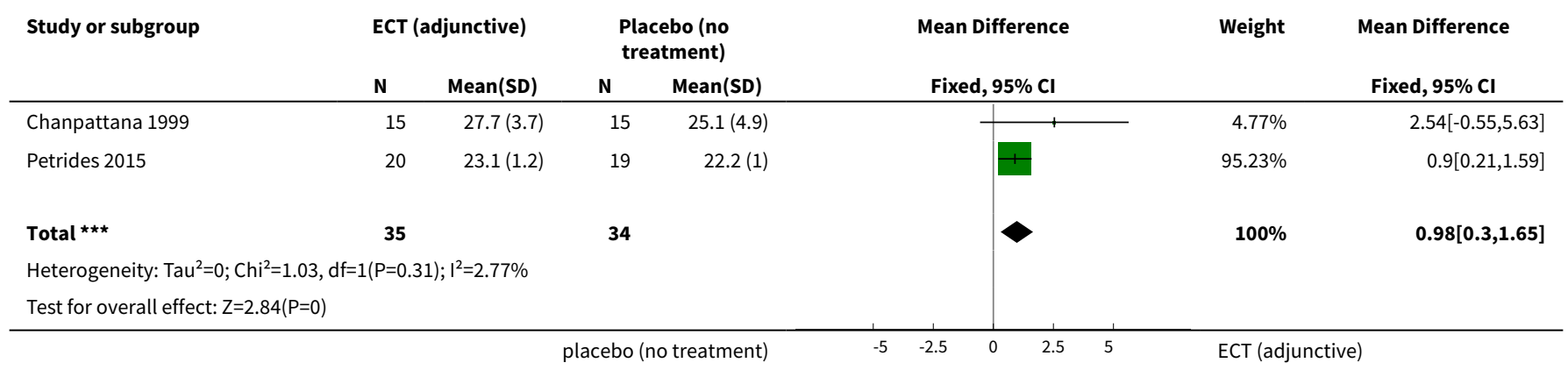




\section{Analysis 3.11. Comparison 3 ECT plus standard care versus standard} care, Outcome 11 Mental state - total score (PANSS, high = poor).

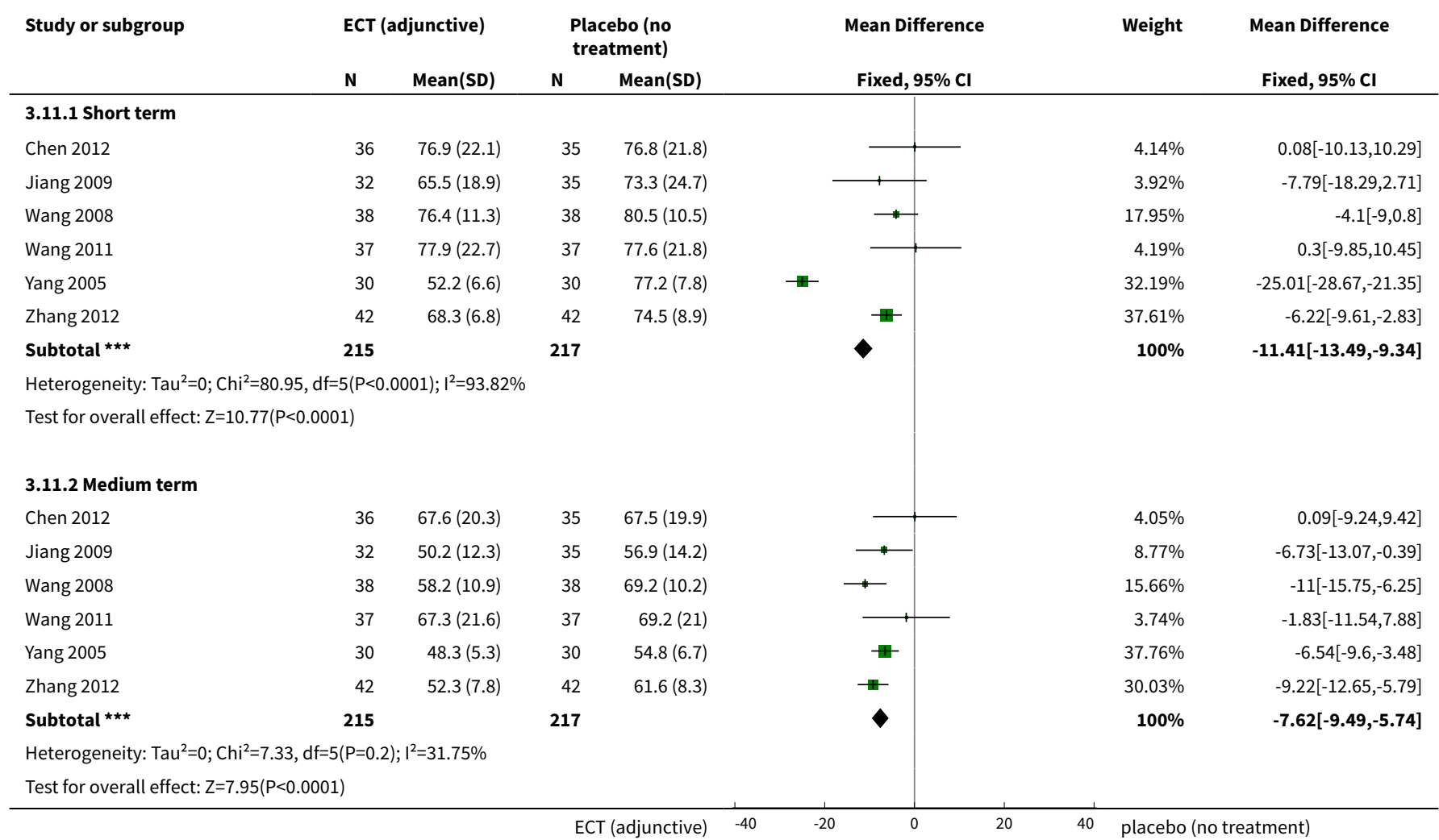

Analysis 3.12. Comparison 3 ECT plus standard care versus standard care, Outcome 12 Mental state - specific symptom score (BPRS, high = poor) - short term.

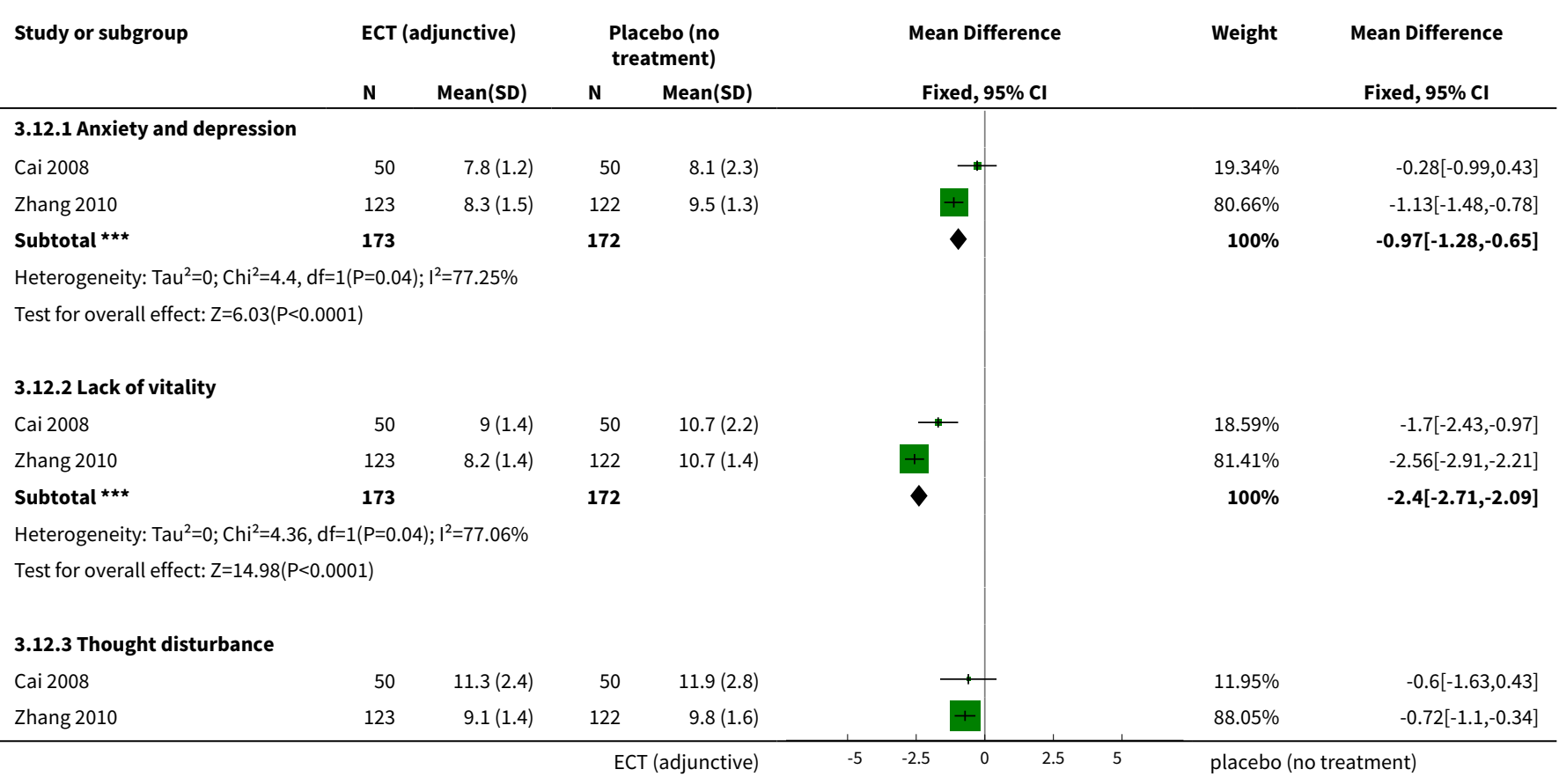




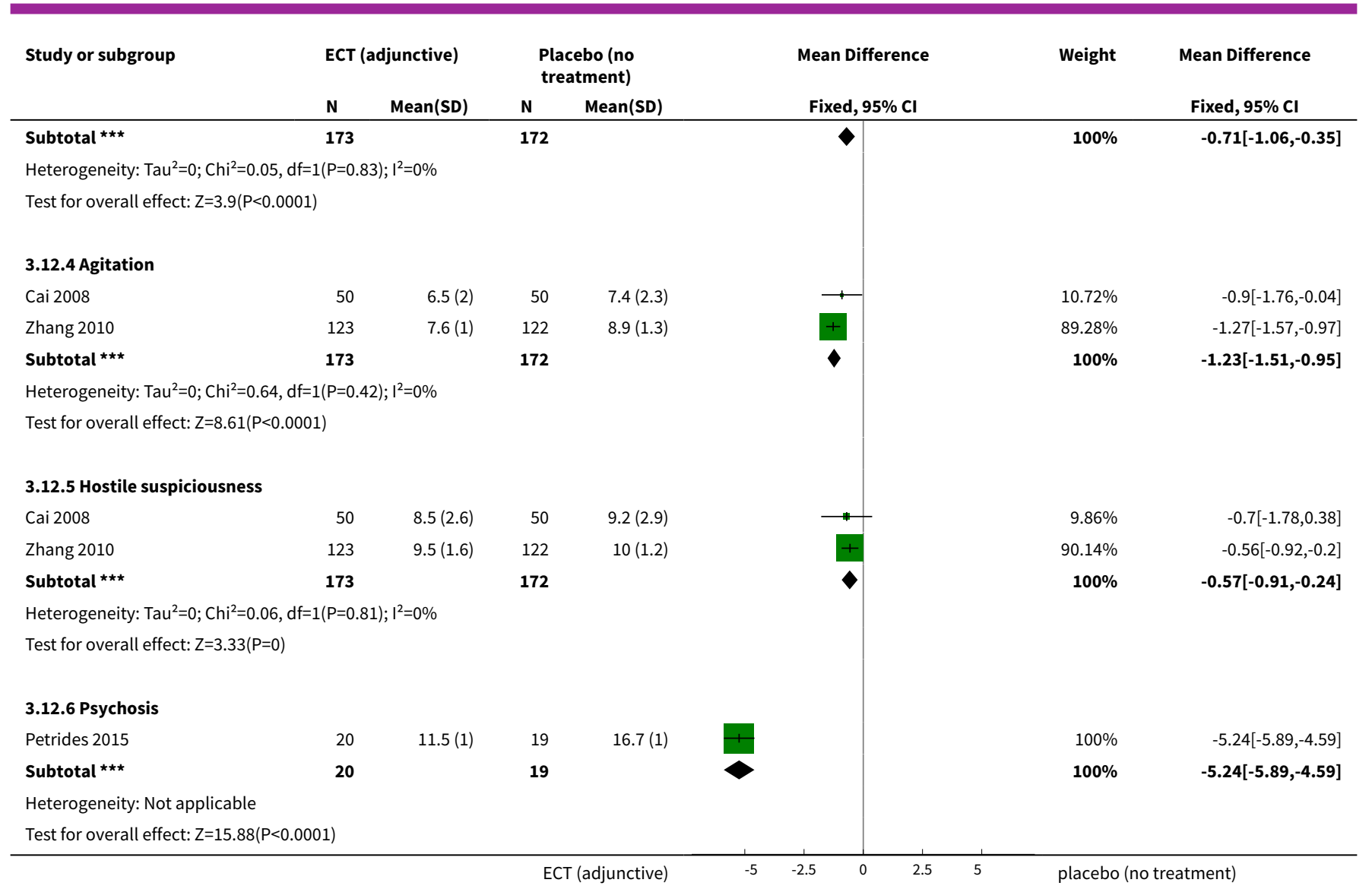

\section{Analysis 3.13. Comparison 3 ECT plus standard care versus standard care, Outcome 13 Mental state - specific symptom score (BPRS, high = poor) - medium term.}

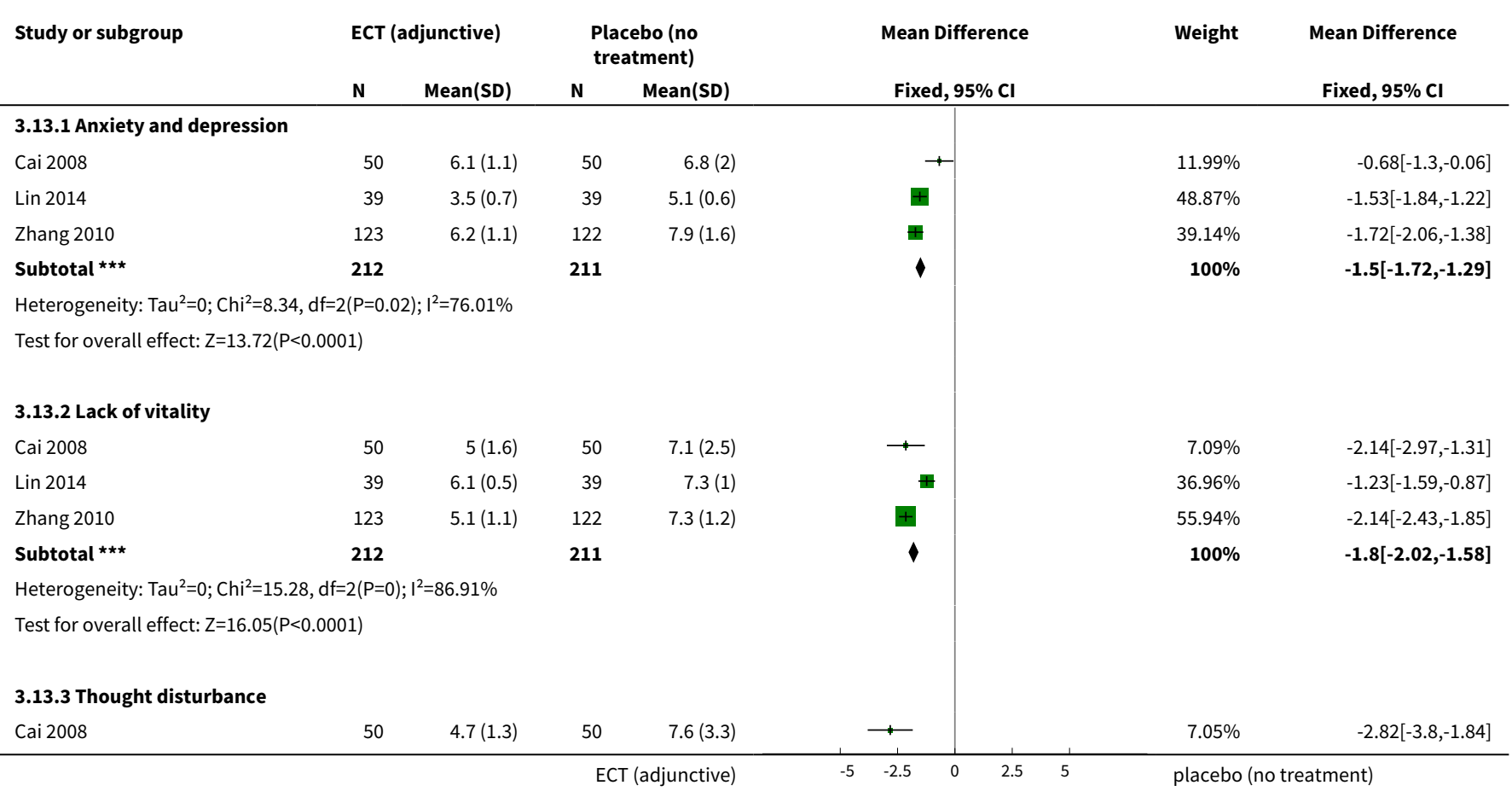




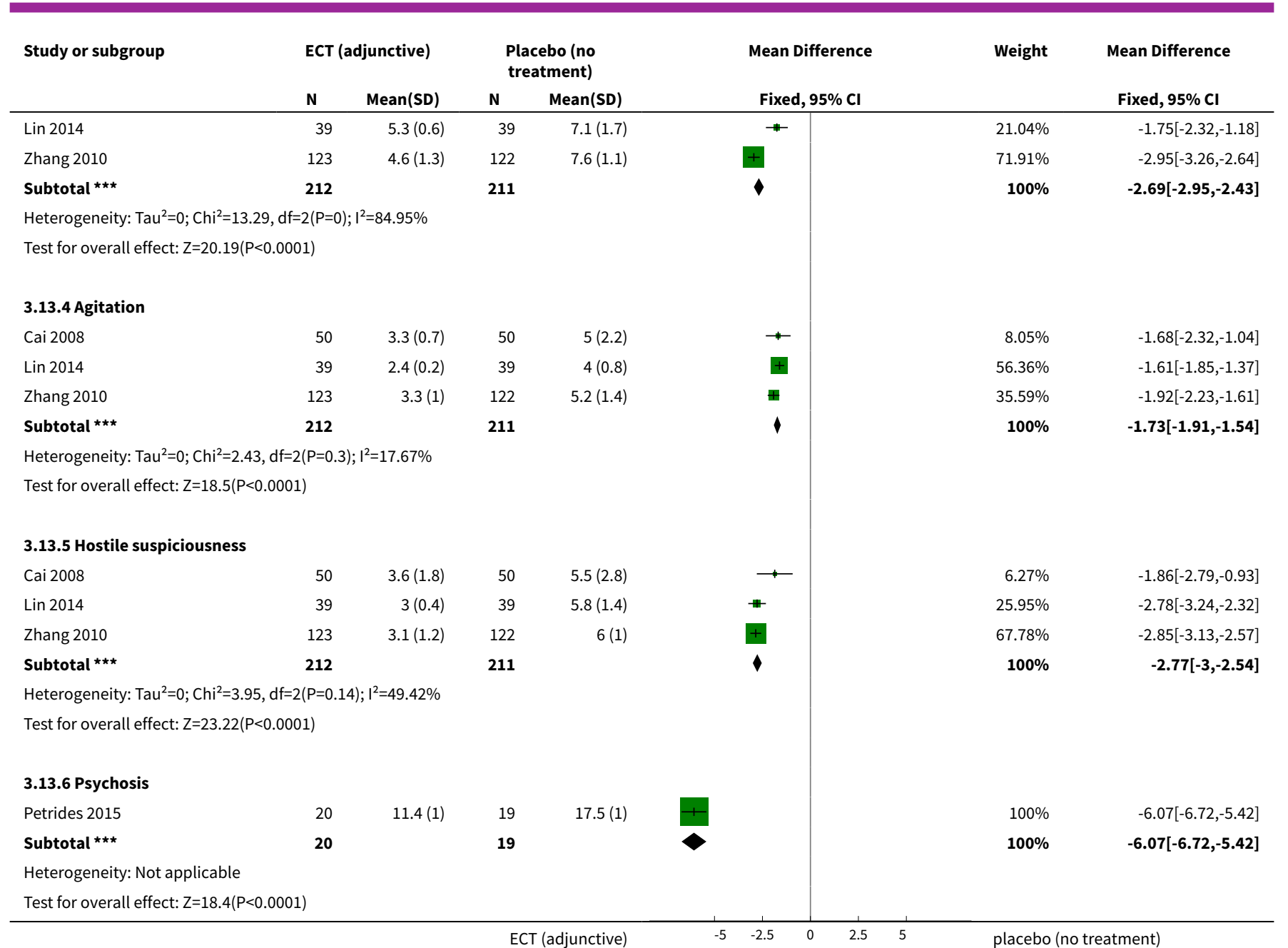

Analysis 3.14. Comparison 3 ECT plus standard care versus standard care, Outcome 14 Mental state - specific symptom score (PANSS, high = poor) - short term.

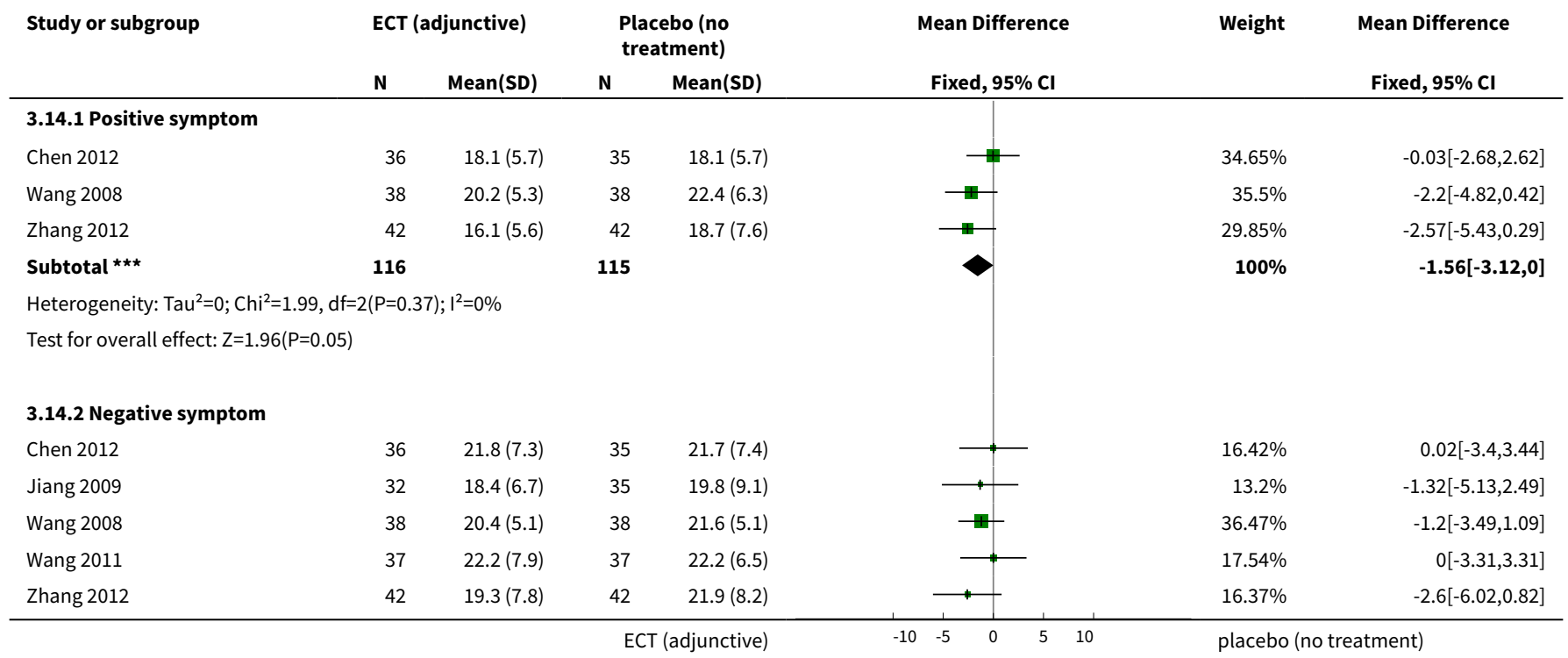




\begin{tabular}{|c|c|c|c|c|c|c|c|}
\hline \multirow[t]{2}{*}{ Study or subgroup } & \multicolumn{2}{|c|}{ ECT (adjunctive) } & \multicolumn{2}{|c|}{$\begin{array}{l}\text { Placebo (no } \\
\text { treatment) }\end{array}$} & \multirow{2}{*}{$\begin{array}{l}\text { Mean Difference } \\
\text { Fixed, } 95 \% \mathrm{Cl}\end{array}$} & \multirow[t]{2}{*}{ Weight } & \multirow{2}{*}{$\begin{array}{c}\text { Mean Difference } \\
\text { Fixed, } 95 \% \mathrm{Cl}\end{array}$} \\
\hline & $\mathbf{N}$ & Mean(SD) & $\mathbf{N}$ & Mean(SD) & & & \\
\hline Subtotal $\star \star \star$ & 185 & & 187 & & & $100 \%$ & $-1.03[-2.42,0.35]$ \\
\hline \multicolumn{8}{|c|}{ Heterogeneity: $\operatorname{Tau}^{2}=0 ; \mathrm{Chi}^{2}=1.59, \mathrm{df}=4(\mathrm{P}=0.81) ; \mathrm{I}^{2}=0 \%$} \\
\hline \multicolumn{8}{|c|}{ Test for overall effect: $Z=1.46(P=0.14)$} \\
\hline \multicolumn{8}{|c|}{ 3.14.3 General psychopathology } \\
\hline Wang 2008 & 38 & $35.8(6.1)$ & 38 & $36.5(7.1)$ & & $56.81 \%$ & $-0.7[-3.68,2.28]$ \\
\hline Wang 2011 & 37 & $34.4(13.7)$ & 37 & $36.5(12.5)$ & & $14.04 \%$ & $-2.05[-8.04,3.94]$ \\
\hline Zhang 2012 & 42 & $31.5(10.2)$ & 42 & $34.6(9.2)$ & & $29.16 \%$ & $-3.09[-7.24,1.06]$ \\
\hline Subtotal $* \star \star$ & 117 & & 117 & & & $100 \%$ & $-1.59[-3.83,0.66]$ \\
\hline \multicolumn{8}{|c|}{ Heterogeneity: $\operatorname{Tau}^{2}=0 ; \mathrm{Chi}^{2}=0.87, \mathrm{df}=2(\mathrm{P}=0.65) ; \mathrm{I}^{2}=0 \%$} \\
\hline \multicolumn{8}{|c|}{ Test for overall effect: $Z=1.39(P=0.17)$} \\
\hline \multicolumn{8}{|l|}{ 3.14.4 Excitement } \\
\hline Yang 2005 & 30 & $7.1(2.8)$ & 30 & $12.9(3.3)$ & & $100 \%$ & $-5.79[-7.34,-4.24]$ \\
\hline Subtotal $\star \star \star$ & 30 & & 30 & & & $100 \%$ & $-5.79[-7.34,-4.24]$ \\
\hline \multicolumn{8}{|c|}{ Heterogeneity: $\mathrm{Tau}^{2}=0 ; \mathrm{Chi}^{2}=0, \mathrm{df}=0(\mathrm{P}<0.0001) ; \mathrm{I}^{2}=100 \%$} \\
\hline \multicolumn{8}{|c|}{ Test for overall effect: $Z=7.31(P<0.0001)$} \\
\hline \multicolumn{8}{|l|}{ 3.14.5 Hostility } \\
\hline Yang 2005 & 30 & $6.5(2.2)$ & 30 & $9(3.4)$ & & $100 \%$ & $-2.48[-3.93,-1.03]$ \\
\hline Subtotal $* \star \star$ & 30 & & 30 & & & $100 \%$ & $-2.48[-3.93,-1.03]$ \\
\hline \multicolumn{8}{|c|}{ Heterogeneity: Not applicable } \\
\hline \multicolumn{8}{|c|}{ Test for overall effect: $Z=3.35(P=0)$} \\
\hline & & & & djunctive) & $\begin{array}{ll}1 & 1 \\
-10 & -5\end{array}$ & placebc & ment) \\
\hline
\end{tabular}

Analysis 3.15. Comparison 3 ECT plus standard care versus standard care, Outcome 15 Mental state - specific symptom score (PANSS, high = poor) - short term (skewed data).

\begin{tabular}{|c|c|c|c|c|}
\hline Study & Intervention & Mean & SD & $\mathbf{N}$ \\
\hline \multicolumn{5}{|c|}{ Positive symptom } \\
\hline Jiang 2009 & $\mathrm{MECT}$ + risperidone & 16.47 & 6.94 & 32 \\
\hline Jiang 2009 & Risperidone alone & 19.11 & 8.12 & 35 \\
\hline Wang 2011 & MECT + clozapine & 18.35 & 6.01 & 37 \\
\hline Wang 2011 & Clozapine alone & 21.34 & 6.11 & 37 \\
\hline \multicolumn{5}{|c|}{ General psychopathology } \\
\hline Chen 2012 & Clozapine alone & 21.75 & 7.36 & 35 \\
\hline Jiang 2009 & MECT + risperidone & 30.12 & 9.23 & 32 \\
\hline Jiang 2009 & Risperidone alone & 34.97 & 10.78 & 35 \\
\hline
\end{tabular}


Analysis 3.16. Comparison 3 ECT plus standard care versus standard care, Outcome 16 Mental state - specific symptom score (PANSS, high = poor) - medium term.

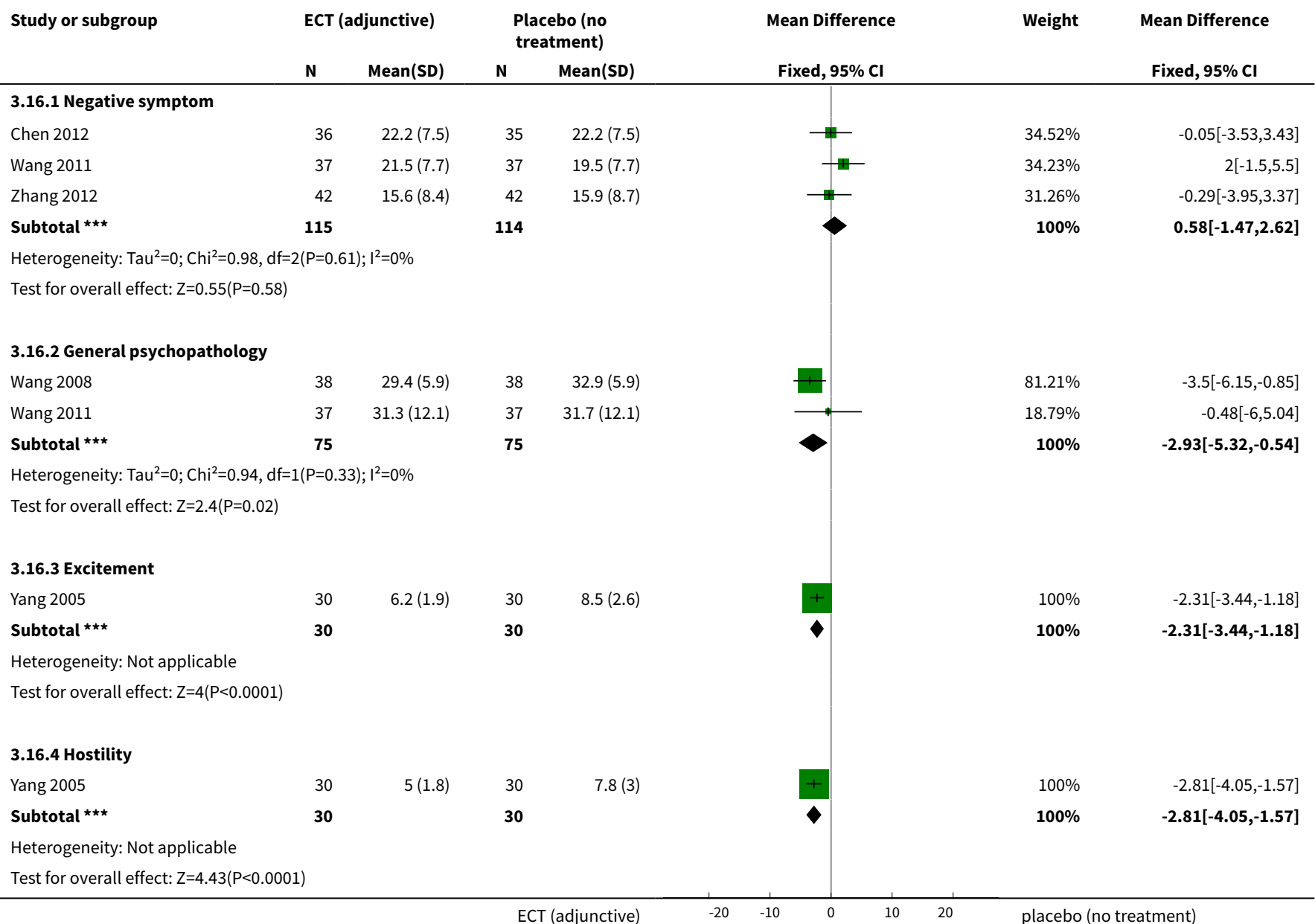

Analysis 3.17. Comparison 3 ECT plus standard care versus standard care, Outcome 17 Mental state - specific symptom score (PANSS, high = poor) - medium term (skewed data).

Mental state - specific symptom score (PANSS, high = poor) - medium term (skewed data)

\begin{tabular}{|c|c|c|c|c|}
\hline Study & Intervention & Mean & SD & $\mathbf{N}$ \\
\hline \multicolumn{5}{|c|}{ Positive symptom } \\
\hline Chen 2012 & MECT + clozapine & 14.24 & 7.10 & 36 \\
\hline Chen 2012 & Clozapine alone & 14.30 & 7.25 & 35 \\
\hline Jiang 2009 & $\mathrm{MECT}+$ risperidone & 10.16 & 4.42 & 32 \\
\hline Jiang 2009 & Risperidone alone & 12.92 & 6.74 & 35 \\
\hline Wang 2008 & $\begin{array}{l}\text { MECT + clozapine and risperi- } \\
\text { done }\end{array}$ & 13.2 & 5.4 & 38 \\
\hline Wang 2008 & clozapine and risperidone & 17.2 & 5.9 & 38 \\
\hline Wang 2011 & MECT + clozapine & 14.98 & 7.62 & 37 \\
\hline Wang 2011 & Clozapine alone & 16.35 & 7.23 & 37 \\
\hline Zhang 2012 & MECT + olanzapine & 12.57 & 7.80 & 42 \\
\hline Zhang 2012 & Olanzapine alone & 15.79 & 6.90 & 42 \\
\hline \multicolumn{5}{|c|}{ Negative symptom } \\
\hline Jiang 2009 & Risperidone alone & 15.33 & 6.73 & 35 \\
\hline
\end{tabular}




\begin{tabular}{|c|c|c|c|c|}
\hline \multicolumn{5}{|c|}{ Mental state - specific symptom score (PANSS, high = poor) - medium term (skewed data) } \\
\hline Study & Intervention & Mean & SD & $\mathbf{N}$ \\
\hline Wang 2008 & $\begin{array}{l}\text { MECT + clozapine and risperi- } \\
\text { done }\end{array}$ & 15.6 & 4.6 & 38 \\
\hline Wang 2008 & clozapine and risperidone & 19.1 & 4.8 & 38 \\
\hline \multicolumn{5}{|c|}{ General psychopathology } \\
\hline Chen 2012 & MECT + clozapine & 31.43 & 12.11 & 36 \\
\hline Chen 2012 & Clozapine alone & 22.2 & 7.34 & 35 \\
\hline Jiang 2009 & MECT + risperidone & 20.74 & 7.16 & 32 \\
\hline Jiang 2009 & Risperidone alone & 27.96 & 8.72 & 35 \\
\hline Zhang 2012 & MECT + olanzapine & 25.31 & 11.1 & 42 \\
\hline Zhang 2012 & Olanzapine alone & 28.17 & 12.5 & 42 \\
\hline
\end{tabular}

Analysis 3.18. Comparison 3 ECT plus standard care versus standard care, Outcome 18 Mental state - specific symptom score (SAPS, high = poor) - short term.

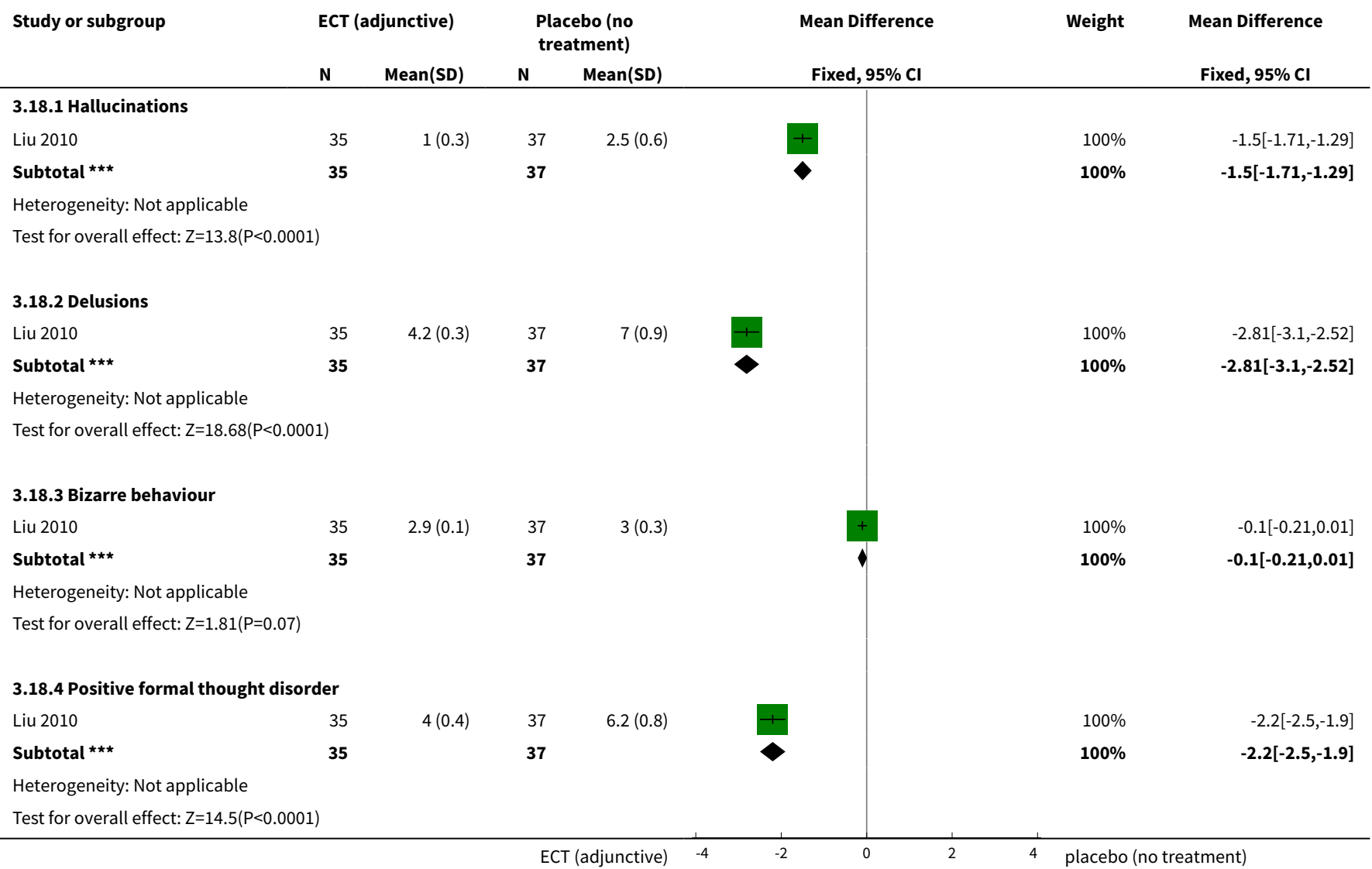

Analysis 3.19. Comparison 3 ECT plus standard care versus standard care, Outcome 19 Mental state - specific symptom score (SANS, high = poor) - short term.

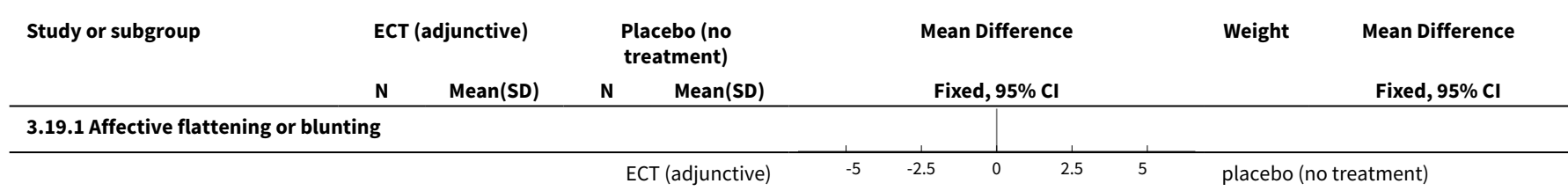




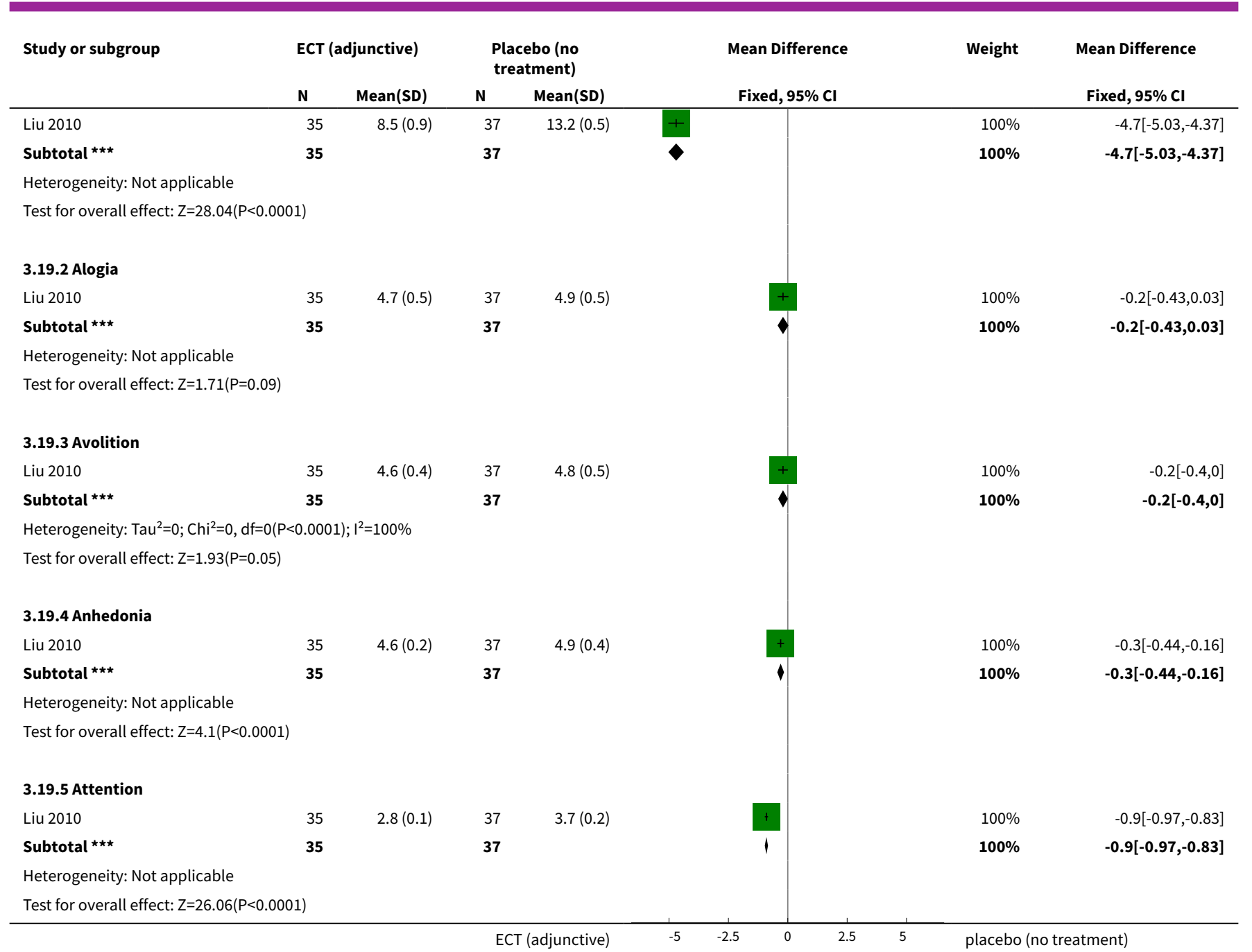

Analysis 3.20. Comparison 3 ECT plus standard care versus standard care, Outcome 20 General functioning - total score (GAF, high = good).

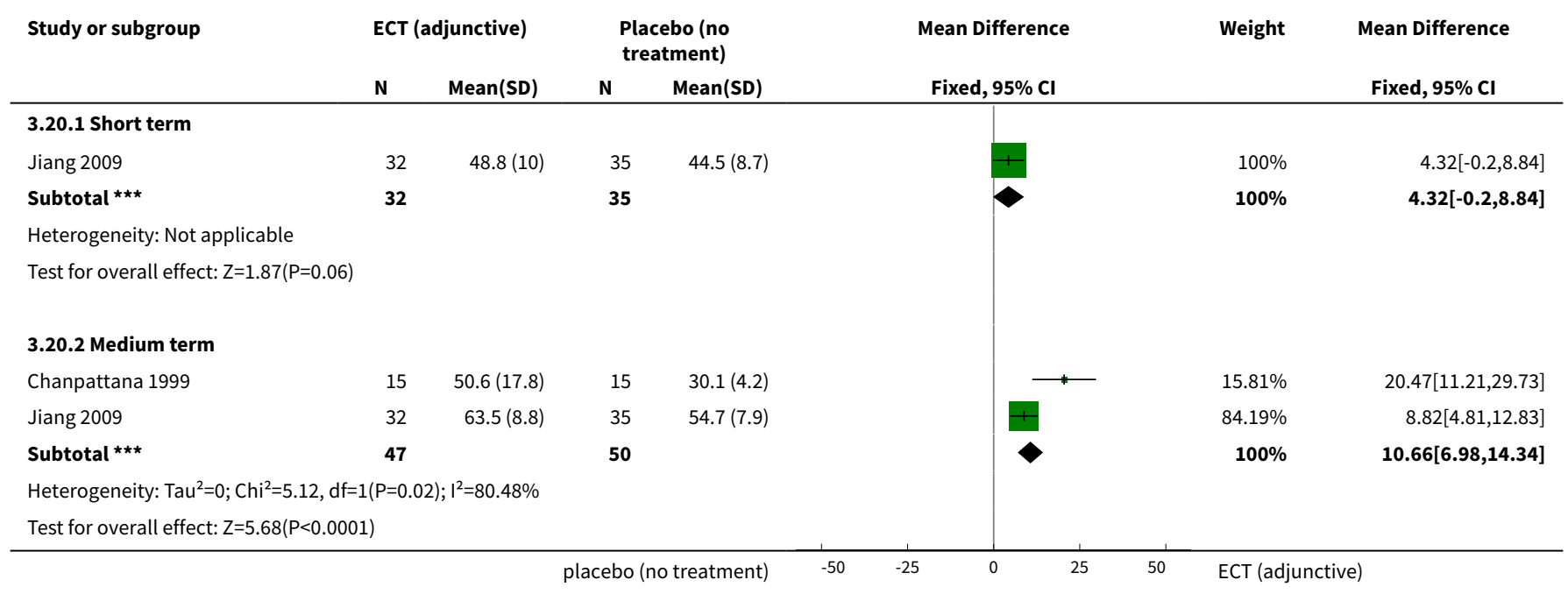


Analysis 3.21. Comparison 3 ECT plus standard care versus standard care, Outcome 21 Adverse events - general - medium term.

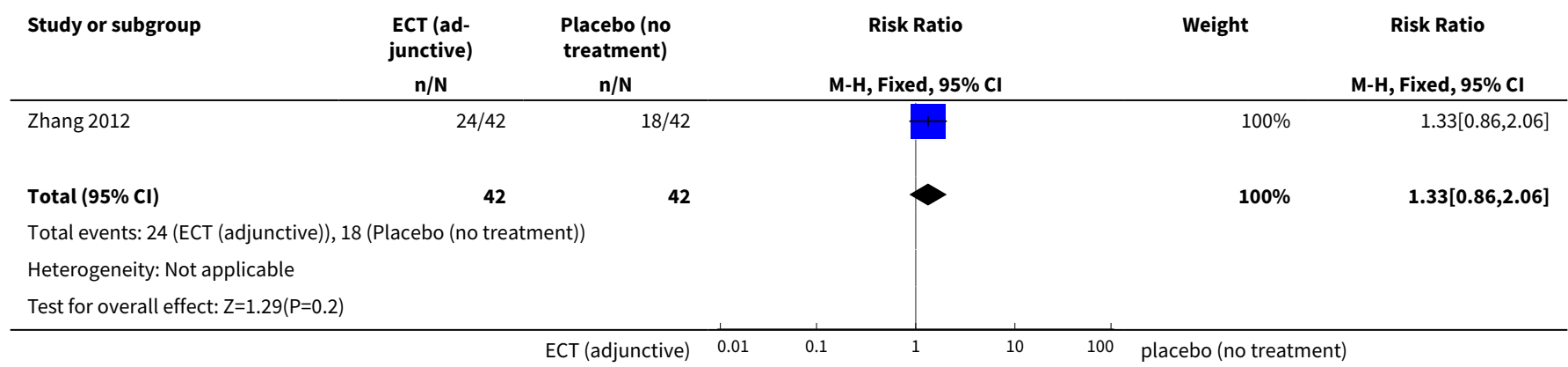

\section{Analysis 3.22. Comparison 3 ECT plus standard care versus standard care, Outcome 22 Adverse events - total score (TESS, high = poor).}

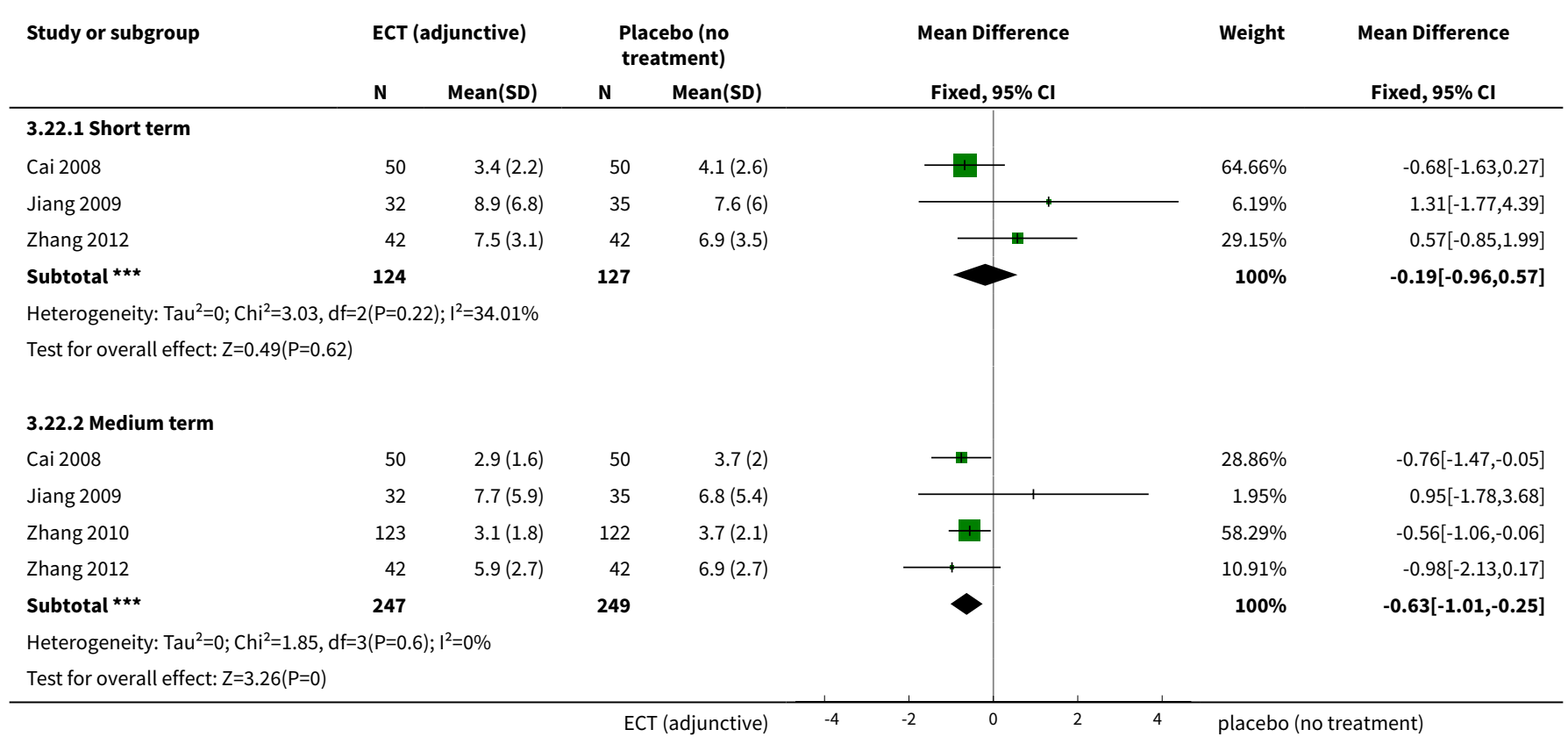

Analysis 3.23. Comparison 3 ECT plus standard care versus standard care, Outcome 23 Adverse events - specific symptom - short term.

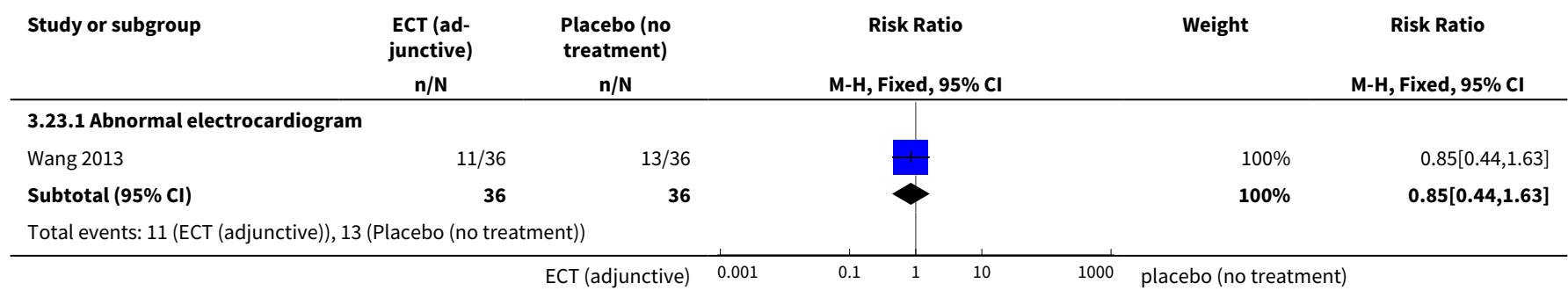




\begin{tabular}{lcccc} 
Study or subgroup & $\begin{array}{c}\text { ECT (ad- } \\
\text { junctive) } \\
\mathrm{n} / \mathrm{N}\end{array}$ & $\begin{array}{c}\text { Placebo (no } \\
\text { treatment) }\end{array}$ & Risk Ratio & Risk Ratio \\
\hline
\end{tabular}

Heterogeneity: Not applicable

Test for overall effect: $\mathrm{Z}=0.5(\mathrm{P}=0.62)$

3.23.2 Abnormal liver function

Wang 2013

Total events: 2 (ECT (adjunctive)), 3 (Placebo (no treatment)) Heterogeneity: Not applicable

Test for overall effect: $Z=0.46(P=0.65)$

\subsubsection{Constipation}

Wang 2013

Total events: 9 (ECT (adjunctive)), 12 (Placebo (no treatment)) Heterogeneity: Not applicable

Test for overall effect: $\mathrm{Z}=0.77(\mathrm{P}=0.44)$

\subsubsection{Headache}

Wang 2013

Total events: 3 (ECT (adjunctive)), 0 (Placebo (no treatment)) Heterogeneity: Not applicable

Test for overall effect: $Z=1.3(P=0.19)$

\subsubsection{Lethargy}

Wang 2013

Total events: 12 (ECT (adjunctive)), 15 (Placebo (no treatment) Heterogeneity: Not applicable

Test for overall effect: $\mathrm{Z}=0.73(\mathrm{P}=0.47)$

\subsubsection{Nausea or vomiting}

Wang 2013

Subtotal $(95 \% \mathrm{CI})$

Total events: 2 (ECT (adjunctive)), 1 (Placebo (no treatment)) Heterogeneity: Not applicable

Test for overall effect: $Z=0.58(P=0.56)$

\subsubsection{Salivation}

Wang 2013

Subtotal $(95 \% \mathrm{Cl})$

Total events: 8 (ECT (adjunctive)), 11 (Placebo (no treatment)) Heterogeneity: Not applicable

Test for overall effect: $\mathrm{Z}=0.8(\mathrm{P}=0.43)$

\subsubsection{Weight gain}

Wang 2013

Subtotal $(95 \% \mathrm{CI})$

36

Total events: 4 (ECT (adjunctive)), 10 (Placebo (no treatment)) Heterogeneity: Not applicable

Test for overall effect: $Z=1.69(P=0.09)$

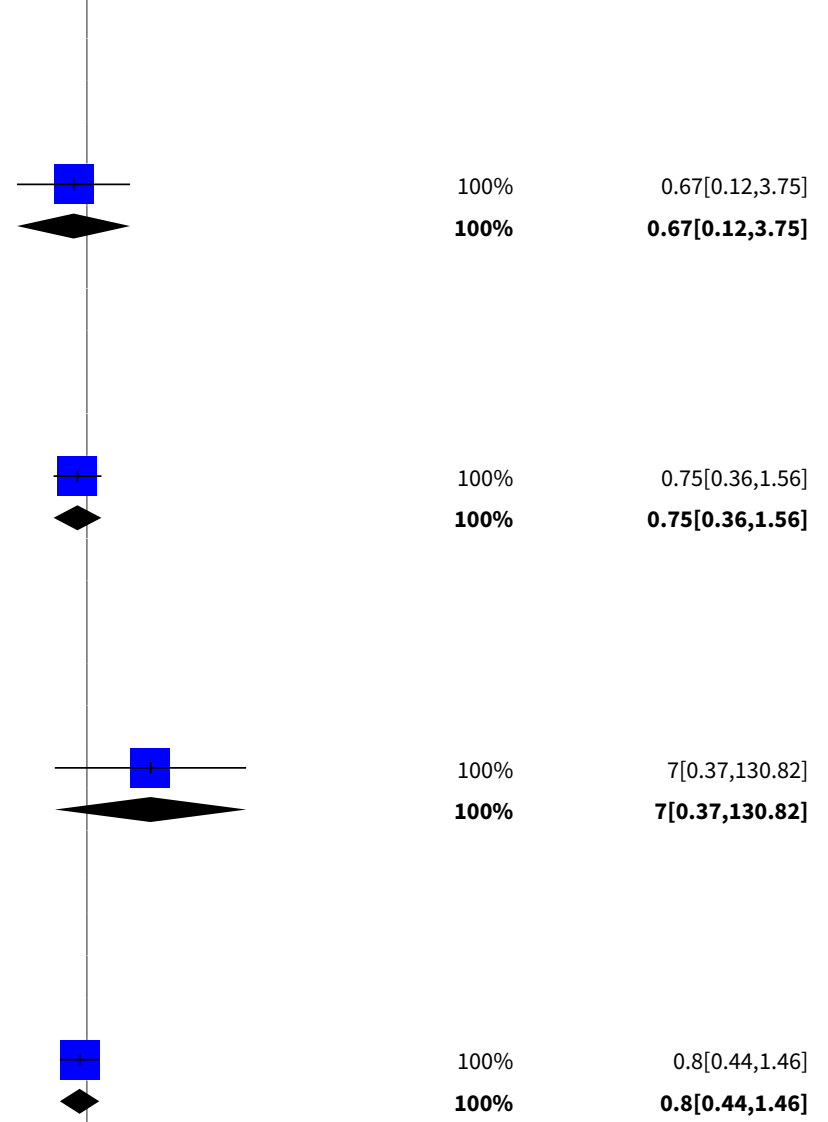

$100 \%$

2[0.19,21.09]

$100 \%$ $2[0.19,21.09]$ 
Analysis 3.24. Comparison 3 ECT plus standard care versus standard care, Outcome 24 Adverse events - specific symptom - medium term.

\begin{tabular}{cccc} 
Study or subgroup & $\begin{array}{c}\text { ECT (ad- } \\
\text { junctive) } \\
\mathrm{n} / \mathrm{N}\end{array}$ & $\begin{array}{c}\text { Placebo (no } \\
\text { treatment) } \\
\mathrm{n} / \mathrm{N}\end{array}$ & Risk Ratio \\
& & $\mathrm{M}-\mathrm{H}, \mathrm{Fixed}, 95 \% \mathrm{Cl}$ \\
\hline
\end{tabular}

Risk Ratio

M-H, Fixed, $95 \% \mathrm{Cl}$

\subsubsection{Abnormal electrocardiogram}

Chen 2012

Jiang 2009

Wang 2011

Yang 2005

Subtotal $(95 \% \mathrm{CI})$
$11 / 36$

$6 / 34$

$11 / 37$

$17 / 30$

137
$12 / 35$

$8 / 35$

$12 / 37$

$17 / 30$

137

Total events: 45 (ECT (adjunctive)), 49 (Placebo (no treatment)) Heterogeneity: $\mathrm{Tau}^{2}=0 ; \mathrm{Chi}^{2}=0.28, \mathrm{df}=3(\mathrm{P}=0.96) ; \mathrm{I}^{2}=0 \%$

Test for overall effect: $Z=0.54(P=0.59)$

\subsubsection{Abnormal electroencephalogram}

Yang 2005

$25 / 30$

Subtotal $(95 \% \mathrm{CI})$

30

$24 / 30$

30

Total events: 25 (ECT (adjunctive)), 24 (Placebo (no treatment))

Heterogeneity: Not applicable

Test for overall effect: $\mathrm{Z}=0.33(\mathrm{P}=0.74)$

3.24.3 Abnormal liver function

Chen 2012

$3 / 36$
$2 / 37$
$3 / 30$
103

Yang 2005

103

Total events: 8 (ECT (adjunctive)), 11 (Placebo (no treatment)) Heterogeneity: $\mathrm{Tau}^{2}=0 ; \mathrm{Chi}^{2}=0.01, \mathrm{df}=2(\mathrm{P}=0.99) ; \mathrm{I}^{2}=0 \%$

Test for overall effect: $Z=0.74(P=0.46)$

\subsubsection{Blurred vision}

Jiang 2009

Subtotal $(95 \% \mathrm{Cl})$

Total events: 4 (ECT (adjunctive)), 4 (Placebo (no treatment)) Heterogeneity: Not applicable

Test for overall effect: $Z=0.04(P=0.97)$

\subsubsection{Breathing discomfort}

Lin 2014

$1 / 39$

Subtotal $(95 \% \mathrm{CI})$

39

$4 / 35$

$3 / 37$

$4 / 30$

102

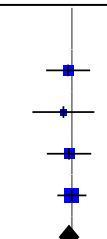

$24.81 \%$

$16.07 \%$

$24.46 \%$

$34.66 \%$

$100 \%$

$100 \%$

$1.04[0.82,1.32]$

$100 \%$

$1.04[0.82,1.32]$

$0.89[0.45,1.75]$

$0.77[0.3,1.99]$

$0.92[0.46,1.81]$

$1[0.64,1.56]$

$0.92[0.67,1.26]$

Total events: 1 (ECT (adjunctive)), 12 (Placebo (no treatment))

Heterogeneity: Not applicable

Test for overall effect: $Z=2.45(P=0.01)$

\subsubsection{Cathisophobia}

Jiang 2009

Subtotal $(95 \% \mathrm{Cl})$

Total events: 7 (ECT (adjunctive)), 9 (Placebo (no treatment)) 


\begin{tabular}{|c|c|c|c|c|c|}
\hline Study or subgroup & $\begin{array}{l}\text { ECT (ad- } \\
\text { junctive) }\end{array}$ & $\begin{array}{l}\text { Placebo (no } \\
\text { treatment) }\end{array}$ & Risk Ratio & Weight & Risk Ratio \\
\hline & $n / N$ & $n / N$ & M-H, Fixed, 95\% Cl & & M-H, Fixed, $95 \% \mathrm{CI}$ \\
\hline
\end{tabular}

\subsubsection{Constipation}

Chen 2012

Wang 2011

Subtotal $(95 \% \mathrm{Cl})$

$16 / 36$

$28 / 37$

73

$28 / 35$
$29 / 37$
7

Total events: 44 (ECT (adjunctive)), 57 (Placebo (no treatment)) Heterogeneity: $\mathrm{Tau}^{2}=0 ; \mathrm{Chi}^{2}=5.84, \mathrm{df}=1(\mathrm{P}=0.02) ; \mathrm{I}^{2}=82.89 \%$

Test for overall effect: $Z=2.41(P=0.02)$

\subsubsection{Decreased activity}

Jiang 2009

Subtotal $(95 \% \mathrm{Cl})$

Total events: 6 (ECT (adjunctive)), 11 (Placebo (no treatment)) Heterogeneity: Not applicable

Test for overall effect: $\mathrm{Z}=1.29(\mathrm{P}=0.2)$

\subsubsection{Dizziness}

Jiang 2009

Subtotal $(95 \% \mathrm{Cl})$

Total events: 10 (ECT (adjunctive)), 6 (Placebo (no treatment)) Heterogeneity: Not applicable

Test for overall effect: $\mathrm{Z}=1.18(\mathrm{P}=0.24)$

\subsubsection{Dry mouth}

Jiang 2009

Subtotal $(95 \% \mathrm{Cl})$

Total events: 5 (ECT (adjunctive)), 5 (Placebo (no treatment)) Heterogeneity: Not applicable

Test for overall effect: $\mathrm{Z}=0.05(\mathrm{P}=0.96)$

\subsubsection{Headache}

Jiang 2009

Lin 2014

Subtotal $(95 \% \mathrm{Cl})$

$9 / 34$
$4 / 39$
73

Total events: 13 (ECT (adjunctive)), 2 (Placebo (no treatment)) Heterogeneity: $\mathrm{Tau}^{2}=0 ; \mathrm{Chi}^{2}=2.24, \mathrm{df}=1(\mathrm{P}=0.13) ; \mathrm{I}^{2}=55.35 \%$

Test for overall effect: $Z=2.53(P=0.01)$

\subsubsection{Indigestion}

Lin 2014

Subtotal $(95 \% \mathrm{Cl})$

Total events: 4 (ECT (adjunctive)), 8 (Placebo (no treatment)) Heterogeneity: Not applicable

Test for overall effect: $Z=1.22(P=0.22)$

\subsubsection{Insomnia}

Jiang 2009

$0 / 34$

Subtotal $(95 \% \mathrm{Cl})$

34

Total events: 0 (ECT (adjunctive)), 10 (Placebo (no treatment))

Heterogeneity: Not applicable

Test for overall effect: $\mathrm{Z}=2.11(\mathrm{P}=0.03)$

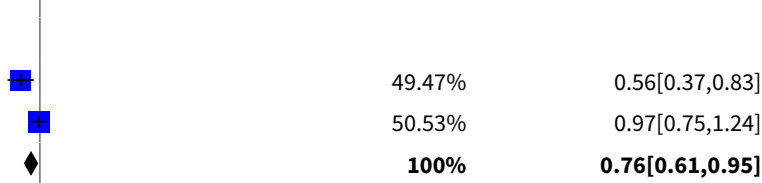

$100 \%$

$0.56[0.23,1.35]$

$100 \%$

$0.56[0.23,1.35]$

$100 \%$

$1.72[0.7,4.2]$

$100 \%$

$1.72[0.7,4.2]$

$100 \%$

$1.03[0.33,3.24]$

$100 \%$

$1.03[0.33,3.24]$
$0 / 35$

2/39

74

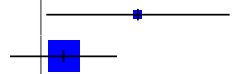

$19.77 \%$

$80.23 \%$

$100 \%$

$19.54[1.18,323.15]$

$2[0.39,10.29]$

$5.47[1.47,20.39]$
$8 / 39$

39 


\begin{tabular}{lcccc} 
Study or subgroup & $\begin{array}{c}\text { ECT (ad- } \\
\text { junctive) }\end{array}$ & $\begin{array}{l}\text { Placebo (no } \\
\text { treatment) } \\
\mathrm{n} / \mathrm{N}\end{array}$ & $\mathrm{n} / \mathrm{N}$ & Risk Ratio \\
\hline
\end{tabular}

\subsubsection{Lethargy}

Chen 2012

Jiang 2009

$19 / 36$

$30 / 35$

Wang 2011

Subtotal $(95 \% \mathrm{CI})$

Total events: 51 (ECT (adjunctive)), 59 (Placebo (no treatment))

Heterogeneity: $\mathrm{Tau}^{2}=0 ; \mathrm{Chi}^{2}=7.32, \mathrm{df}=2(\mathrm{P}=0.03) ; \mathrm{I}^{2}=72.68 \%$

Test for overall effect: $Z=1.49(P=0.14)$

\subsubsection{Leucopenia}

Chen 2012

Wang 2011

Subtotal $(95 \% \mathrm{Cl})$

$3 / 36$
$0 / 37$

73

$4 / 35$

$0 / 37$

72

Total events: 3 (ECT (adjunctive)), 4 (Placebo (no treatment))

Heterogeneity: Not applicable

Test for overall effect: $\mathrm{Z}=0.44(\mathrm{P}=0.66)$

\subsubsection{Muscle rigidity}

Jiang 2009

Subtotal $(95 \% \mathrm{Cl})$

34

Total events: 9 (ECT (adjunctive)), 12 (Placebo (no treatment)) Heterogeneity: Not applicable

Test for overall effect: $\mathrm{Z}=0.7(\mathrm{P}=0.48)$

\subsubsection{Nausea or vomiting}

Jiang 2009

Lin 2014

Subtotal $(95 \% \mathrm{Cl})$

73

Total events: 7 (ECT (adjunctive)), 9 (Placebo (no treatment)) Heterogeneity: $\mathrm{Tau}^{2}=0 ; \mathrm{Chi}^{2}=2.48, \mathrm{df}=1(\mathrm{P}=0.12) ; \mathrm{I}^{2}=59.6 \%$ Test for overall effect: $\mathrm{Z}=0.49(\mathrm{P}=0.62)$

3.24.18 Recurrence of pre-existing involuntary 'jerky' movements Petrides 2015

Total events: 1 (ECT (adjunctive)), 0 (Placebo (no treatment)) Heterogeneity: Not applicable

Test for overall effect: $\mathrm{Z}=0.65(\mathrm{P}=0.51)$

\subsubsection{Salivation}

Chen 2012

Total events: 31 (ECT (adjunctive)), 38 (Placebo (no treatment)) Heterogeneity: $\mathrm{Tau}^{2}=0 ; \mathrm{Chi}^{2}=0.86, \mathrm{df}=1(\mathrm{P}=0.35) ; \mathrm{I}^{2}=0 \%$

Test for overall effect: $\mathrm{Z}=1.23(\mathrm{P}=0.22)$

\subsubsection{Tremor}

Jiang 2009

Subtotal $(95 \% \mathrm{Cl})$

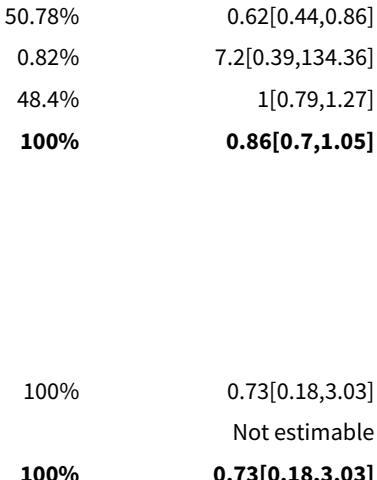

$100 \%$

$0.77[0.37,1.59]$

$100 \%$

$0.77[0.37,1.59]$
$33.01 \%$

$66.99 \%$

$100 \%$

$100 \%$

$2.86[0.12,66.11]$

$100 \%$

$2.86[0.12,66.11]$
$1.72[0.44,6.63]$

$0.33[0.07,1.55]$

$0.79[0.31,2.02]$

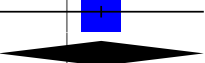


Total events: 7 (ECT (adjunctive)), 10 (Placebo (no treatment))

Heterogeneity: Not applicable

Test for overall effect: $\mathrm{Z}=0.76(\mathrm{P}=0.45)$

\subsubsection{Weight gain}

Chen 2012

Jiang 2009

Wang 2011

Subtotal $(95 \%$ C $)$

Total events: 24 (ECT (adjunctive)), 33 (Placebo (no treatment)) Heterogeneity: $\mathrm{Tau}^{2}=0 ; \mathrm{Chi}^{2}=1.64, \mathrm{df}=2(\mathrm{P}=0.44) ; \mathrm{I}^{2}=0 \%$

Test for overall effect: $Z=1.41(P=0.16)$

\subsubsection{Others}

Chen 2012

Wang 2011

Subtotal $(95 \% \mathrm{CI})$

$\begin{array}{rr}4 / 36 & 4 / 35 \\ 6 / 37 & 6 / 37 \\ \mathbf{7 3} & \mathbf{7 2}\end{array}$

Total events: 10 (ECT (adjunctive)), 10 (Placebo (no treatment))

Heterogeneity: $\mathrm{Tau}^{2}=0 ; \mathrm{Chi}^{2}=0, \mathrm{df}=1(\mathrm{P}=0.97) ; \mathrm{I}^{2}=0 \%$

Test for overall effect: $\mathrm{Z}=0.03(\mathrm{P}=0.98)$

$\begin{array}{lllllll}\text { ECT (adjunctive) } & 0.001 & 0.1 & 1 & 10 & 1000 & \text { placebo (no treatment) }\end{array}$

Analysis 3.25. Comparison 3 ECT plus standard care versus standard care, Outcome 25 Adverse events - specific symptom score (TESS, high = poor) - short term (skewed data).

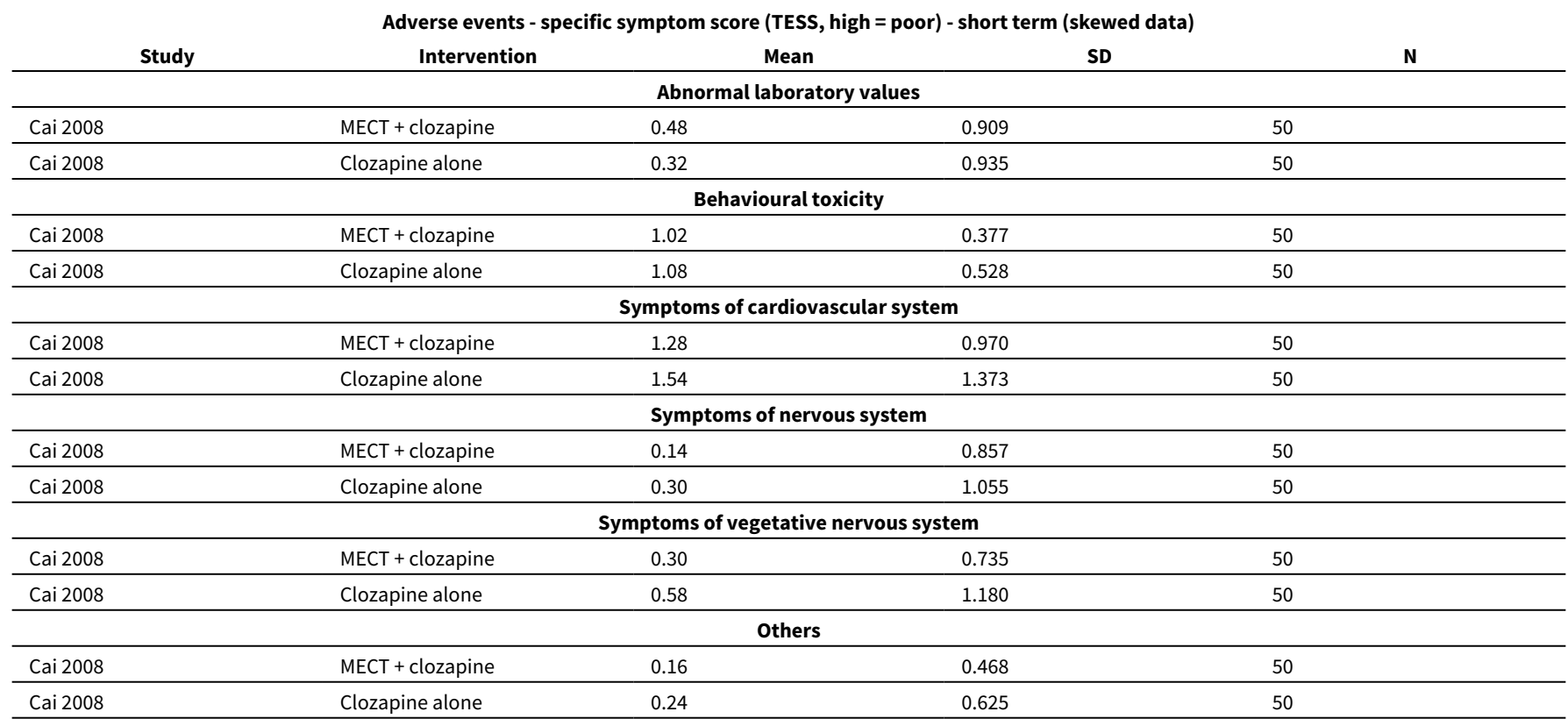


Analysis 3.26. Comparison 3 ECT plus standard care versus standard care, Outcome 26 Adverse events - specific symptom score (TESS, high = poor) - medium term.

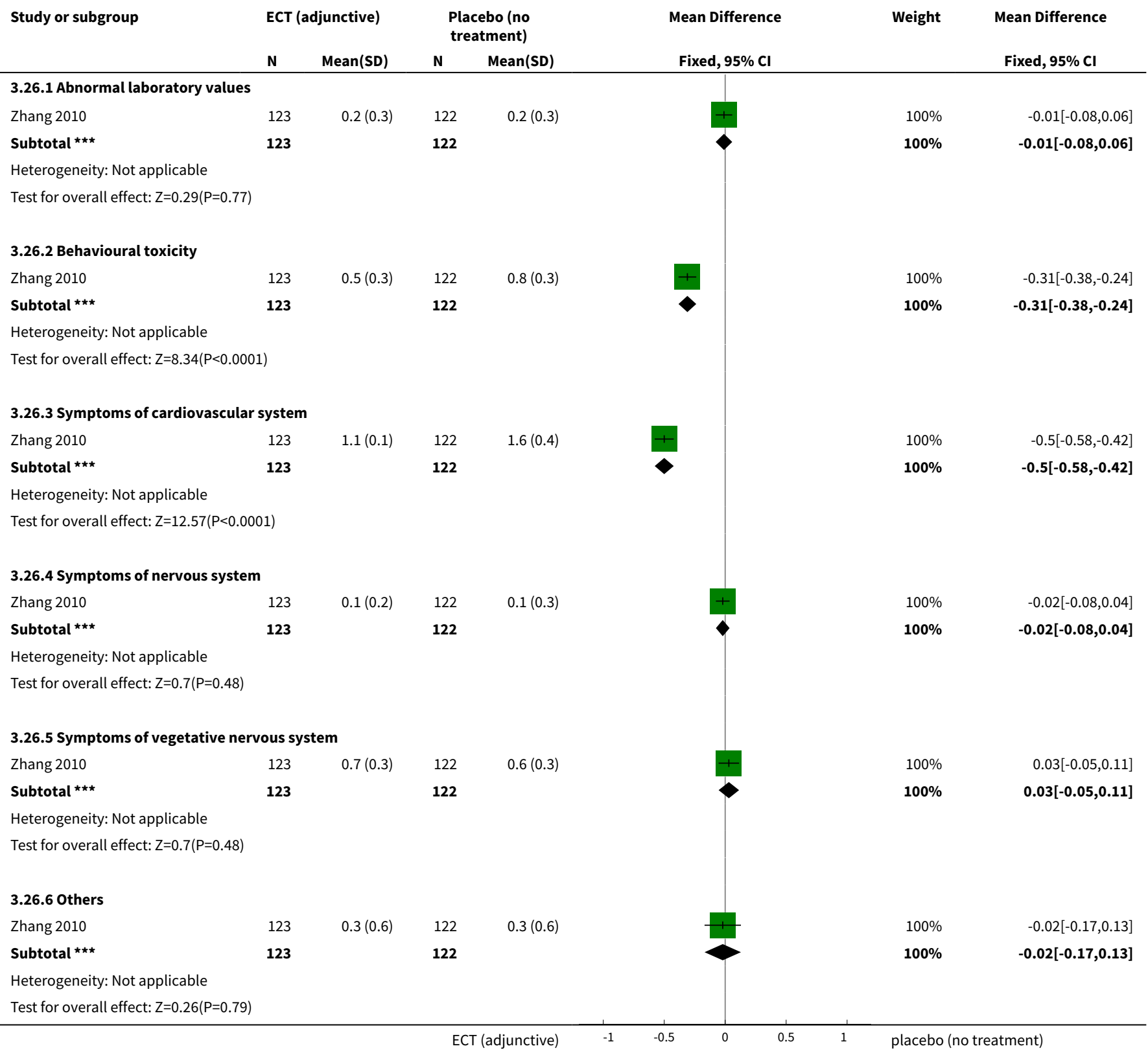

Analysis 3.27. Comparison 3 ECT plus standard care versus standard care, Outcome 27 Adverse events - specific symptom score (TESS, high = poor) - medium term (skewed data).

Adverse events - specific symptom score (TESS, high = poor) - medium term (skewed data)

\begin{tabular}{|c|c|c|c|c|}
\hline Study & Intervention & Mean & SD & $\mathbf{N}$ \\
\hline \multicolumn{5}{|c|}{ Abnormal laboratory values } \\
\hline Cai 2008 & MECT + clozapine & 0.22 & 0.679 & 50 \\
\hline Cai 2008 & Clozapine alone & 0.22 & 0.545 & 50 \\
\hline \multicolumn{5}{|c|}{ Behavioral toxicity } \\
\hline Cai 2008 & MECT + clozapine & 0.96 & 0.402 & 50 \\
\hline Cai 2008 & Clozapine alone & 0.94 & 0.373 & 50 \\
\hline
\end{tabular}

Electroconvulsive therapy for treatment-resistant schizophrenia (Review) 


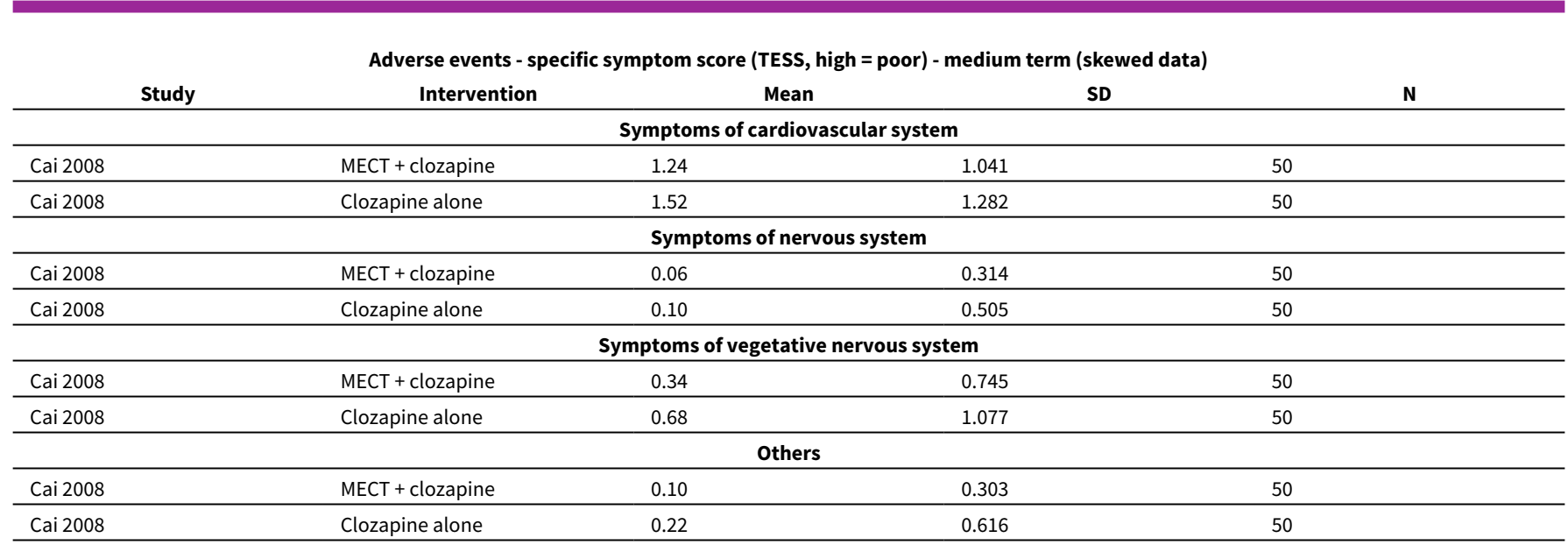

\section{Comparison 4. ECT alone versus antipsychotic alone (flupenthixol)}

\begin{tabular}{lllll}
\hline Outcome or subgroup title & $\begin{array}{l}\text { No. of } \\
\text { studies }\end{array}$ & $\begin{array}{l}\text { No. of } \\
\text { partici- } \\
\text { pants }\end{array}$ & Statistical method & Effect size \\
\hline $\begin{array}{l}1 \text { Mental state - total score (BPRS, high = poor) - } \\
\text { medium term }\end{array}$ & 30 & $\begin{array}{l}\text { Mean Difference (IV, Fixed, 95\% } \\
\text { Cl) }\end{array}$ & -0.93 [-6.95, 5.09] \\
\hline $\begin{array}{l}2 \text { Mental state - total score (MMSE, high = good) - } \\
\text { medium term }\end{array}$ & 30 & $\begin{array}{l}\text { Mean Difference (IV, Fixed, 95\% } \\
\text { Cl) }\end{array}$ & -0.20 [-3.70, 3.30] \\
\hline $\begin{array}{l}3 \text { General functioning - total score (GAF, high }= \\
\text { good) - medium term }\end{array}$ & 1 & 30 & $\begin{array}{l}\text { Mean Difference (IV, Fixed, 95\% } \\
\text { Cl) }\end{array}$ & -0.66 [-3.60, 2.28] \\
\hline
\end{tabular}

Analysis 4.1. Comparison $4 \mathrm{ECT}$ alone versus antipsychotic alone (flupenthixol), Outcome 1 Mental state - total score (BPRS, high = poor) - medium term.

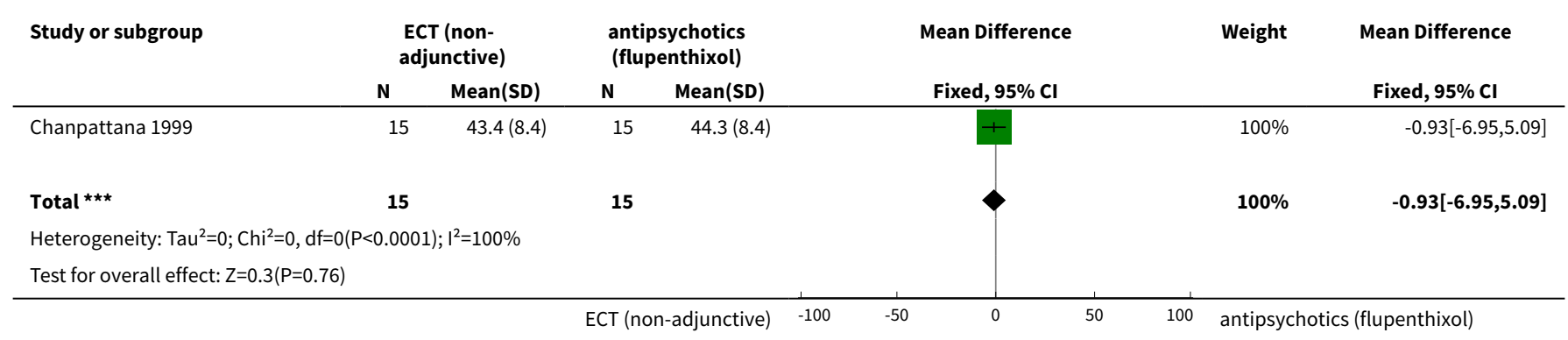


Analysis 4.2. Comparison $4 \mathrm{ECT}$ alone versus antipsychotic alone (flupenthixol), Outcome 2 Mental state - total score (MMSE, high = good) - medium term.

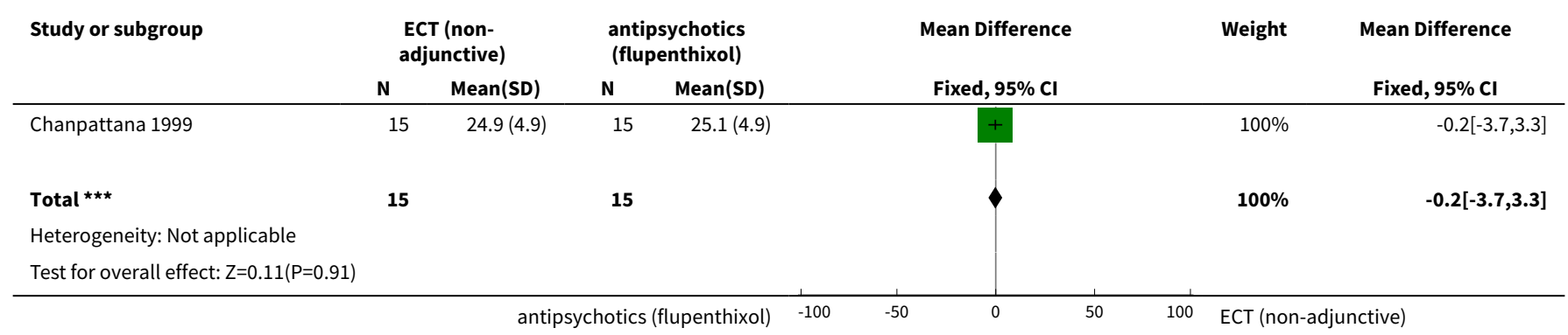

Analysis 4.3. Comparison $4 \mathrm{ECT}$ alone versus antipsychotic alone (flupenthixol), Outcome 3 General functioning - total score (GAF, high = good) - medium term.

\begin{tabular}{|c|c|c|c|c|c|c|c|c|}
\hline \multirow[t]{2}{*}{ Study or subgroup } & \multicolumn{2}{|c|}{$\begin{array}{l}\text { ECT (non- } \\
\text { adjunctive) }\end{array}$} & \multicolumn{2}{|c|}{$\begin{array}{l}\text { antipsychotics } \\
\text { (flupenthixol) }\end{array}$} & \multirow{2}{*}{\multicolumn{2}{|c|}{$\begin{array}{l}\text { Mean Difference } \\
\text { Fixed, } 95 \% \mathrm{Cl}\end{array}$}} & \multirow[t]{2}{*}{ Weight } & \multirow{2}{*}{$\begin{array}{l}\text { Mean Difference } \\
\text { Fixed, } 95 \% \mathrm{Cl}\end{array}$} \\
\hline & $\mathbf{N}$ & Mean(SD) & $\mathbf{N}$ & Mean(SD) & & & & \\
\hline Chanpattana 1999 & 15 & $29.5(4.1)$ & 15 & $30.1(4.2)$ & & + & $100 \%$ & $-0.66[-3.6,2.28]$ \\
\hline 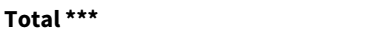 & 15 & & 15 & & & $>$ & $100 \%$ & $-0.66[-3.6,2.28]$ \\
\hline \multicolumn{9}{|l|}{ Heterogeneity: Not applicable } \\
\hline & & ant & otics & penthixol) & -100 & -50 & 100 ECT (no & ctive) \\
\hline
\end{tabular}

Comparison 5. SUBGROUP: ECT plus standard care versus standard care (TEST FOR DIFFERENCE BETWEEN SHORT/ LONG COURSE OF ECT)

\begin{tabular}{|c|c|c|c|c|}
\hline Outcome or subgroup title & $\begin{array}{l}\text { No. of } \\
\text { studies }\end{array}$ & $\begin{array}{l}\text { No. of } \\
\text { partici- } \\
\text { pants }\end{array}$ & Statistical method & Effect size \\
\hline $\begin{array}{l}1 \text { Response to treatment - clinically signifi- } \\
\text { cant response (as defined by each study) - } \\
\text { medium term }\end{array}$ & 9 & & Risk Ratio (M-H, Fixed, 95\% Cl) & Subtotals only \\
\hline 1.1 Short course of ECT & 6 & 450 & Risk Ratio (M-H, Fixed, 95\% Cl) & $2.23[1.78,2.80]$ \\
\hline 1.2 Long course of ECT & 3 & 369 & Risk Ratio (M-H, Fixed, 95\% Cl) & $1.87[1.49,2.36]$ \\
\hline
\end{tabular}


Analysis 5.1. Comparison 5 SUBGROUP: ECT plus standard care versus standard care (TEST FOR DIFFERENCE BETWEEN SHORT/LONG COURSE OF ECT), Outcome 1 Response to treatment - clinically significant response (as defined by each study) - medium term.

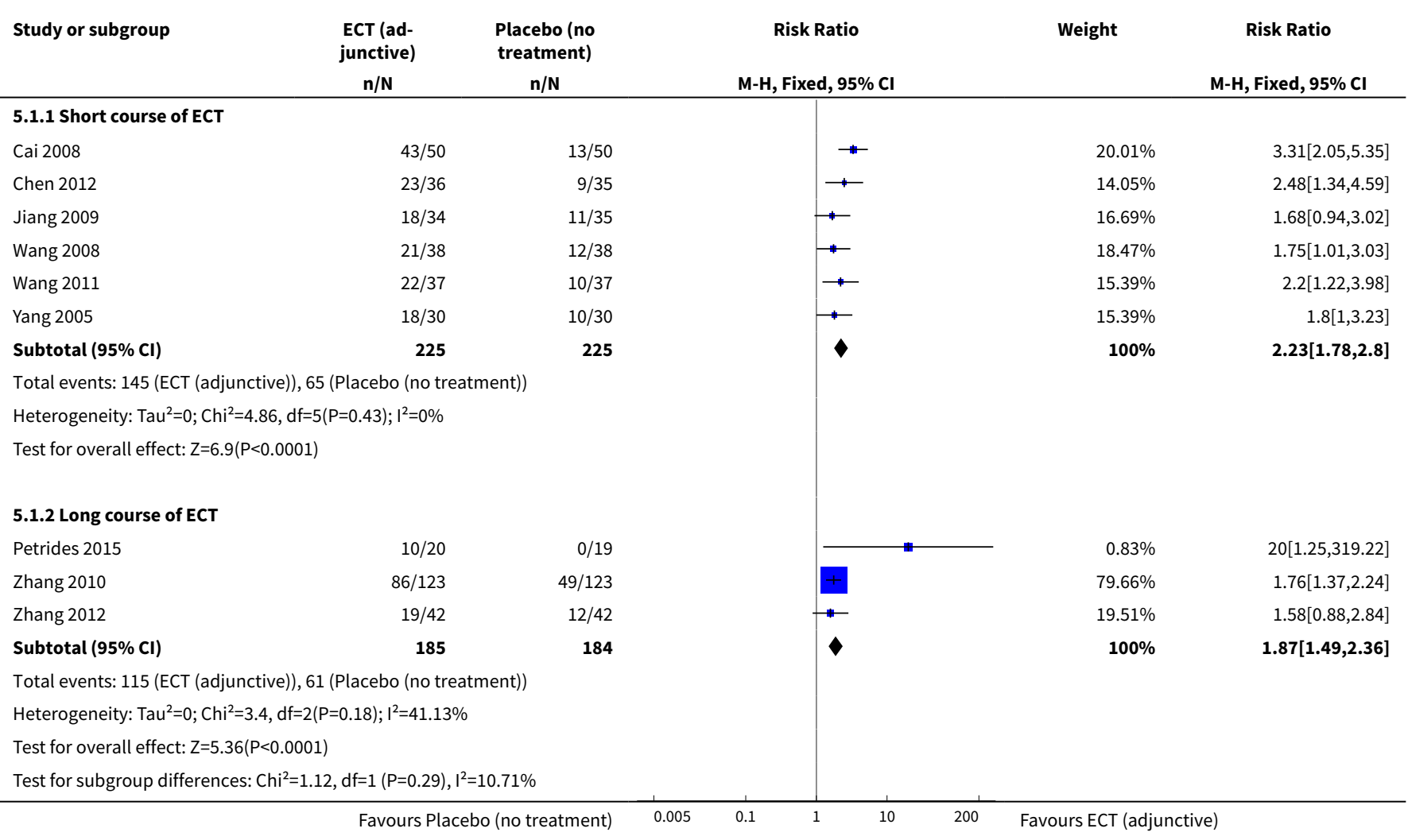

Comparison 6. SENSITIVITY ANALYSIS: ECT plus standard care versus standard care (ASSUMPTIONS FOR LOST BINARY DATA)

\begin{tabular}{lllll}
\hline Outcome or subgroup title & $\begin{array}{l}\text { No. of } \\
\text { studies }\end{array}$ & $\begin{array}{l}\text { No. of } \\
\text { partici- } \\
\text { pants }\end{array}$ & Statistical method & Effect size \\
\hline $\begin{array}{l}1 \text { Response to treatment - clinically signifi- } \\
\text { cant response (as defined by each study) } \\
\text { medium term }\end{array}$ & 9 & & Risk Ratio (M-H, Fixed, 95\% Cl) & Subtotals only \\
\hline $\begin{array}{lllll}1.1 \text { With assumption } \\
1.2 \text { Without assumption }\end{array}$ & 9 & 819 & Risk Ratio (M-H, Fixed, 95\% Cl) & $2.06[1.75,2.42]$ \\
\hline
\end{tabular}


Analysis 6.1. Comparison 6 SENSITIVITY ANALYSIS: ECT plus standard care versus standard care (ASSUMPTIONS FOR LOST BINARY DATA), Outcome 1 Response to treatment - clinically significant response (as defined by each study) - medium term.

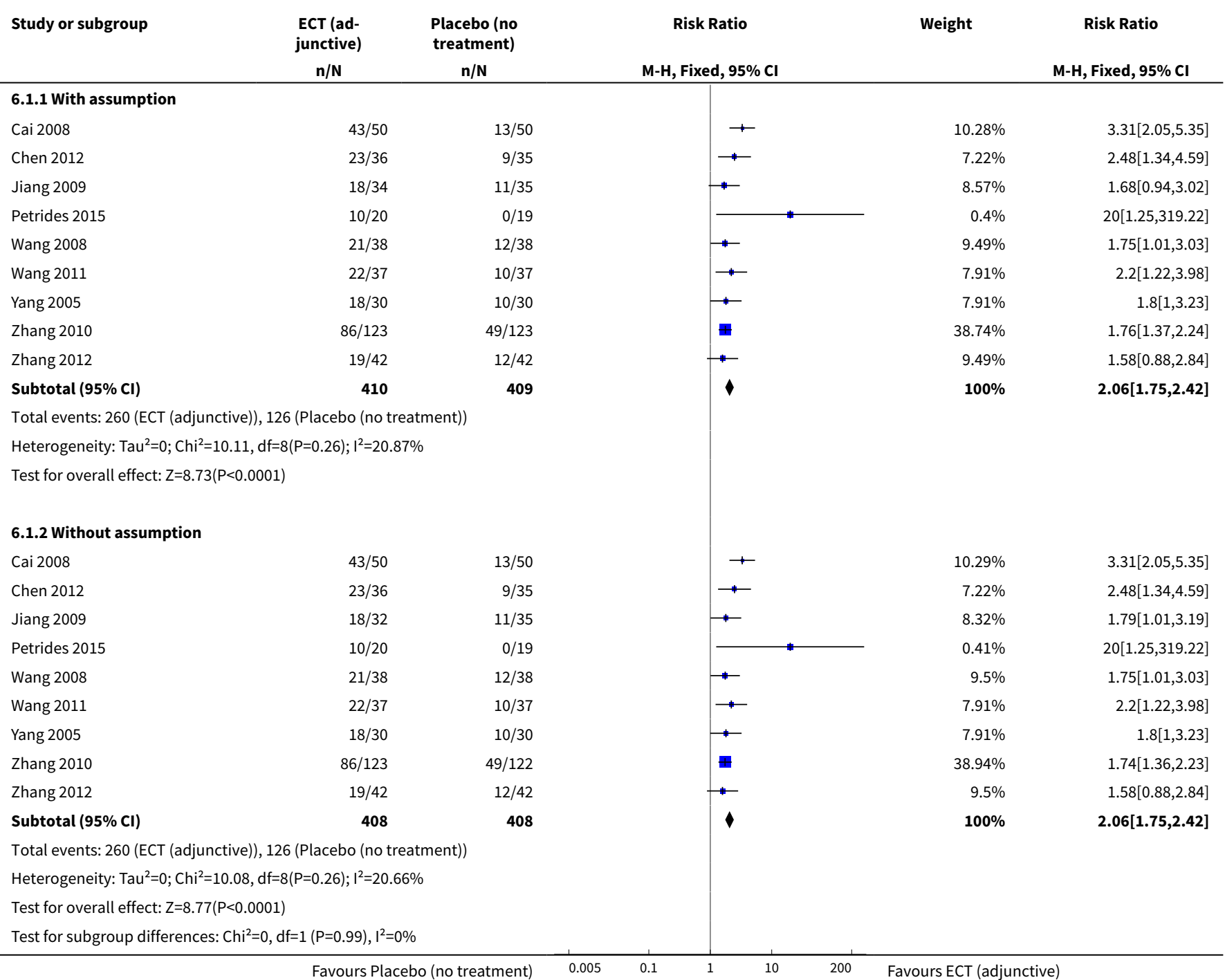

Comparison 7. SENSITIVITY ANALYSIS: ECT plus standard care versus standard care (FIXED-EFFECT AND RANDOMEFFECTS MODEL)

\begin{tabular}{|c|c|c|c|c|}
\hline Outcome or subgroup title & $\begin{array}{l}\text { No. of } \\
\text { studies }\end{array}$ & $\begin{array}{l}\text { No. of } \\
\text { partici- } \\
\text { pants }\end{array}$ & Statistical method & Effect size \\
\hline $\begin{array}{l}1 \text { Response to treatment - clinically signifi- } \\
\text { cant response (as defined by each study) - } \\
\text { medium term }\end{array}$ & 9 & & Risk Ratio (M-H, Fixed, 95\% Cl) & Subtotals only \\
\hline 1.1 Fixed-effect model & 9 & 819 & Risk Ratio (M-H, Fixed, 95\% Cl) & $2.06[1.75,2.42]$ \\
\hline 1.2 Random-effects model & 9 & 819 & Risk Ratio (M-H, Fixed, 95\% Cl) & $2.06[1.75,2.42]$ \\
\hline
\end{tabular}


Analysis 7.1. Comparison 7 SENSITIVITY ANALYSIS: ECT plus standard care versus standard care (FIXED-EFFECT AND RANDOM-EFFECTS MODEL), Outcome 1 Response to treatment - clinically significant response (as defined by each study) - medium term.

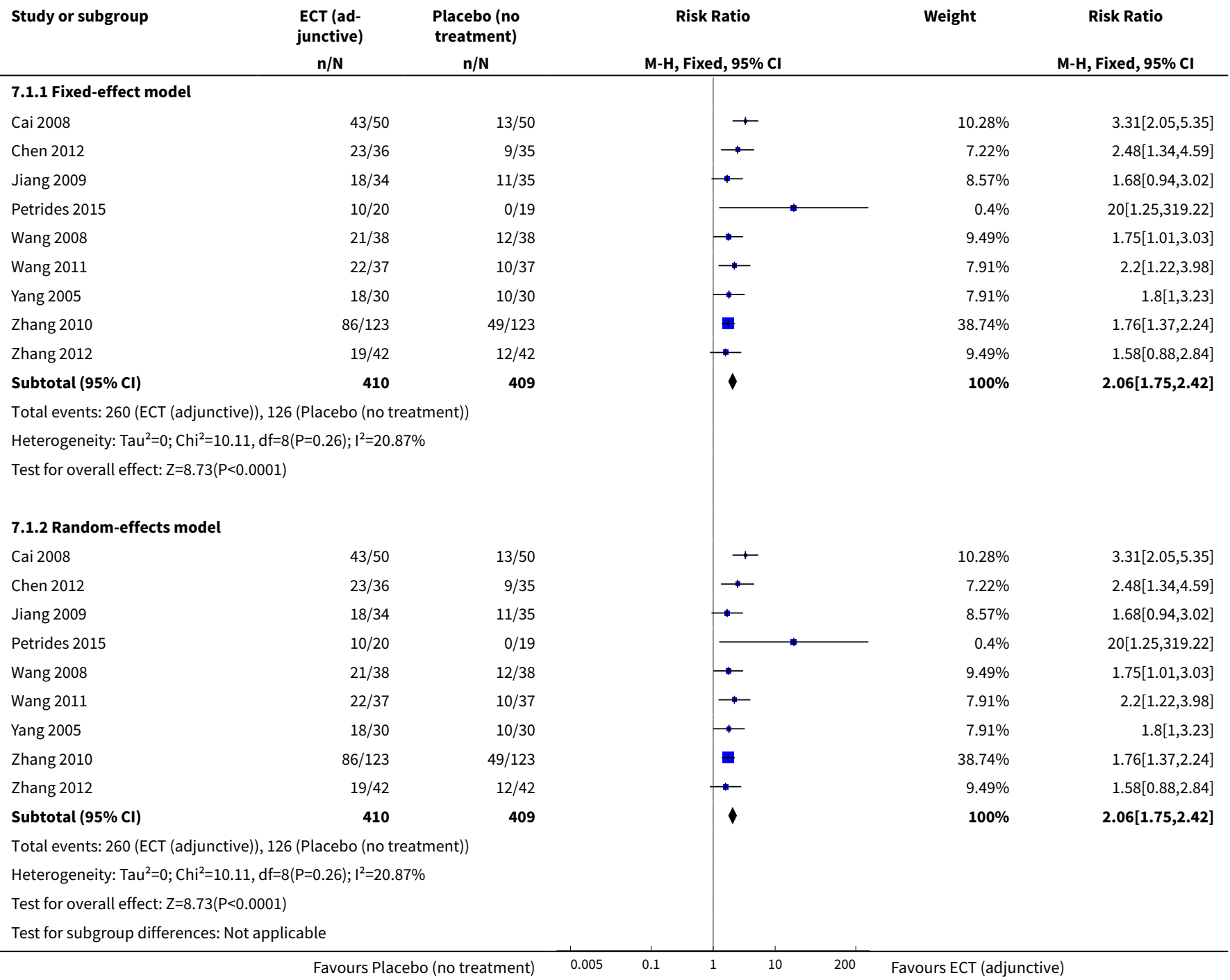

\section{CONTRIBUTIONS OF AUTHORS}

Diarmid Sinclair: protocol development, study selection, data collection, and review writing

Sai Zhao: study selection, data collection, reviewed and drafted parts of the review

Fang Qi: study selection, data collection, and review writing

Kazare Nyakyoma: reviewed and drafted parts of the review

Joey Kwong: reviewed the review

Clive Adams: protocol development, advisor for review 


\section{DECLARATIONS OF INTEREST}

Diarmid Sinclair: none known.

Sai Zhao: none known.

Fang Qi: none known.

Kazare Nyakyoma: none known.

Joey Kwong: none known.

Clive Adams: none known.

\section{SOURCES OF SUPPORT}

\section{Internal sources}

- University of Nottingham, UK.

The University of Nottingham hosts the Cochrane Schizophrenia Group and provided support in conducting the electronic searches, obtaining the studies, and administrative and editorial support.

\section{External sources}

- National Institute for Health Research, UK.

This review is the supported the National Institute for Health Research (NIHR) through the 'NIHR Cochrane Incentive Scheme 2017', and received 5000GBP grant (grant number 17/62/26). This review presents independent research funded by the NIHR. The views expressed are those of the authors and not necessarily those of the NHS, NIHR, or the Department of Health.

\section{DIFFERENCES BETWEEN PROTOCOLANDREVIEW}

We have reordered the outcomes to reflect the order of outcomes reported in the 'Summary of findings' tables. We have also used the longest follow-up time point available for presenting in the 'Summary of findings' tables.

We have updated the Methods to reflect the latest changes in Cochrane Schizophrenia's template, which includes updates to the Subgroup analysis and investigation of heterogeneity section (2. Investigation of heterogeneity).

\section{NOTES}

None.

\section{INDEX TERMS}

\section{Medical Subject Headings (MeSH)}

Antipsychotic Agents [therapeutic use]; Brief Psychiatric Rating Scale; Electroconvulsive Therapy [ ${ }^{\star}$ adverse effects] [methods]; Flupenthixol [therapeutic use]; Memory Disorders [ ${ }^{*}$ etiology]; Patient Readmission [statistics \& numerical data]; Piperazines [therapeutic use]; Randomized Controlled Trials as Topic; Schizophrenia [ ${ }^{\star}$ therapy]; Standard of Care; Thiazoles [therapeutic use]; Treatment Outcome

\section{MeSH check words}

Adult; Female; Humans; Male 